\title{
Organotypic and Microphysiological Human Tissue Models for Drug Discovery and Development-Current State-of-the-Art and Future Perspectives
}

Sonia Youhanna, Aurino M. Kemas, Lena Preiss, Yitian Zhou, Joanne X. Shen, Selgin D. Cakal, Francesco S. Paqualini, Sravan K. Goparaju, Reza Zandi Shafagh, Johan Ulrik Lind, Carl M. Sellgren, and Volker M. Lauschke

Department of Physiology and Pharmacology, Karolinska Institutet, Stockholm, Sweden (S.Y., A.M.K., L.P., Y.Z., J.X.S., S.K.G., R.Z.S., C.M.S., V.M.L.); Department of Drug Metabolism and Pharmacokinetics (DMPK), Merck KGaA, Darmstadt, Germany (L.P.); Department of Health Technology, Technical University of Denmark, Lyngby, Denmark (S.D.C., J.U.L.); Synthetic Physiology Laboratory, Department of Civil Engineering and Architecture, University of Pavia, Pavia, Italy (F.S.P.); Division of Micro- and Nanosystems, KTH Royal Institute of Technology, Stockholm, Sweden (Z.S.); and Dr Margarete Fischer-Bosch Institute of Clinical Pharmacology, Stuttgart, Germany (V.M.L.)

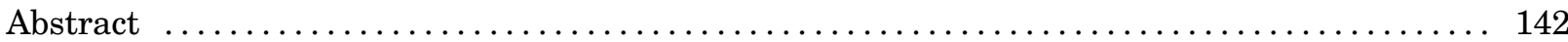

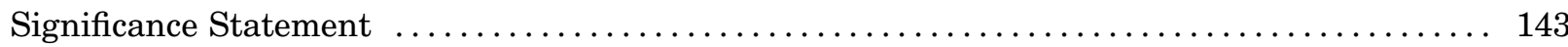

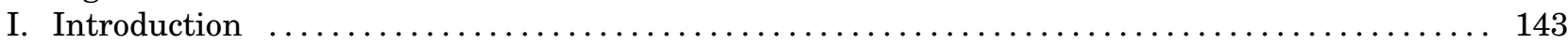

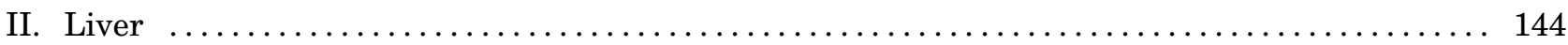

A. Critical Overview of Available Hepatic Cell Models $\ldots \ldots \ldots \ldots \ldots \ldots \ldots \ldots \ldots \ldots \ldots \ldots 144$

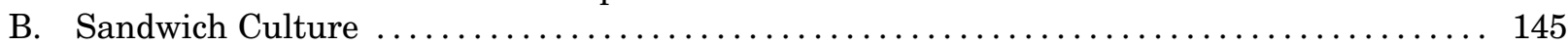

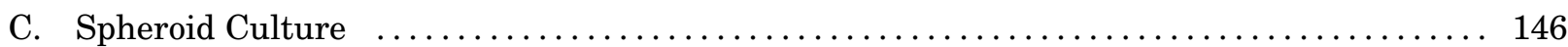

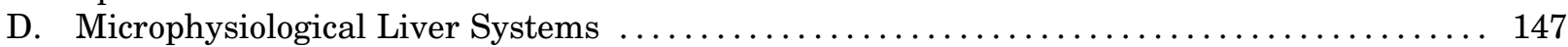

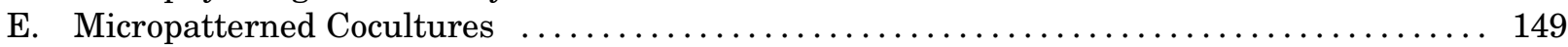

F. Main Applications of Organotypic Liver Models $\ldots \ldots \ldots \ldots \ldots \ldots \ldots \ldots \ldots \ldots \ldots \ldots \ldots$

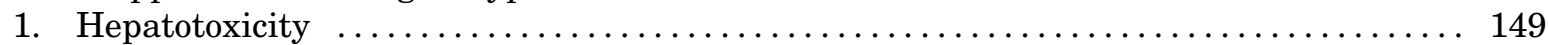

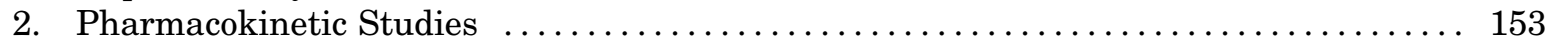

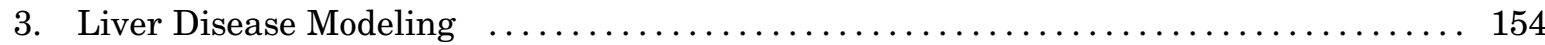

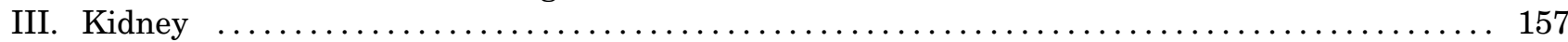

A. Models of the Major Nephrotic Segments $\ldots \ldots \ldots \ldots \ldots \ldots \ldots \ldots \ldots \ldots \ldots \ldots \ldots \ldots \ldots$

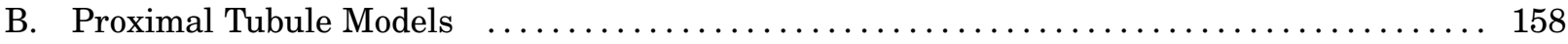

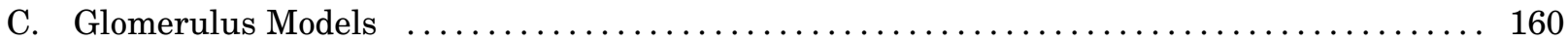

D. Distal Tubule and Collecting Duct Models $\ldots \ldots \ldots \ldots \ldots \ldots \ldots \ldots \ldots \ldots \ldots \ldots \ldots \ldots \ldots$

E. Applications of 3D Human Kidney Models $\ldots \ldots \ldots \ldots \ldots \ldots \ldots \ldots \ldots \ldots \ldots \ldots \ldots \ldots 1$

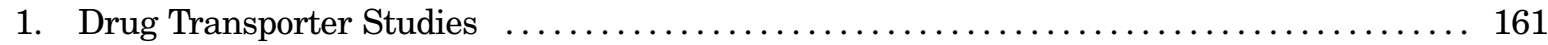

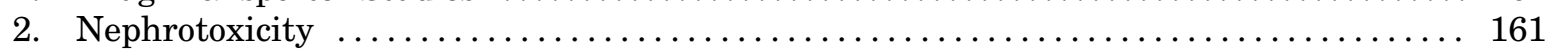

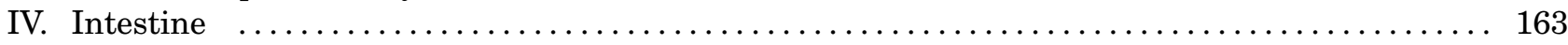

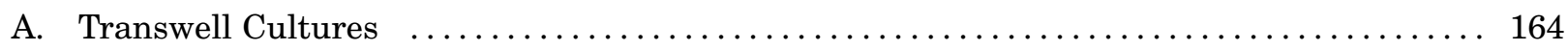

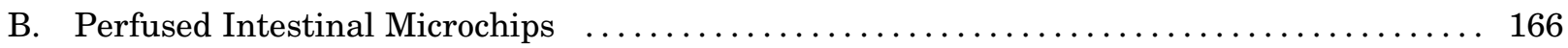

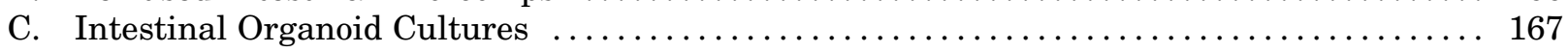

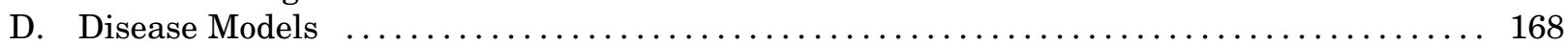

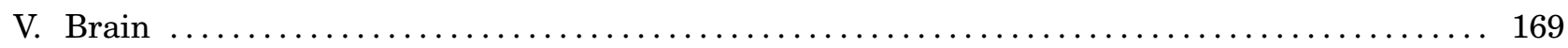

A. Deriving Brain Cells from Pluripotent Stem Cells $\ldots \ldots \ldots \ldots \ldots \ldots \ldots \ldots \ldots \ldots \ldots 169$

B. Organotypic and Microphysiological Human 3D Tissue Models of the Brain .......... 169

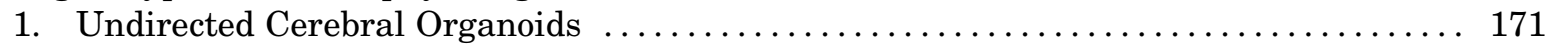

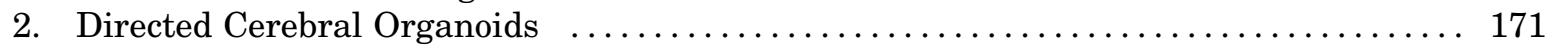

Address correspondence to: Volker Lauschke, Department of Physiology and Pharmacology, Karolinska Institutet, Solnavägen 9, 17177 Stockholm, Sweden. E-mail: volker.lauschke@ki.se

This work was supported by the Swedish Research Council [Grants 2016-01153, 2016-01154, and 2019-01837]; by the EU/EFPIA/OICR/ McGill/KTH/Diamond Innovative Medicines Initiative 2 Joint Undertaking [Grant 875510]; by the Knut and Alice Wallenberg Foundation [Grant VC-2021-0026]; by Merck KGaA; and by the Robert Bosch Foundation.

Y.Z. and V.M.L. are cofounders and shareholders of PersoMedix AB. In addition, V.M.L. is CEO and shareholder of HepaPredict AB and discloses consultancy work for Enginzyme $\mathrm{AB}$. The other authors declare no conflicts of interest.

dx.doi.org/10.1124/pharmrev.120.000238. 
3. Neural Spheroids

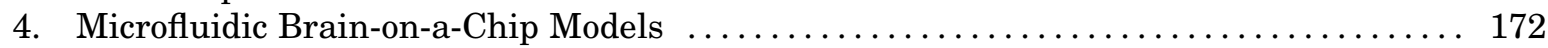

C. Applications of Organotypic Brain Models $\ldots \ldots \ldots \ldots \ldots \ldots \ldots \ldots \ldots \ldots \ldots \ldots \ldots \ldots \ldots$

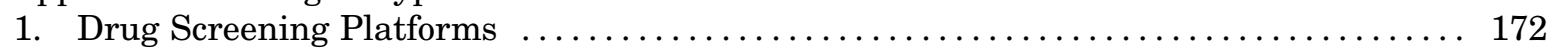

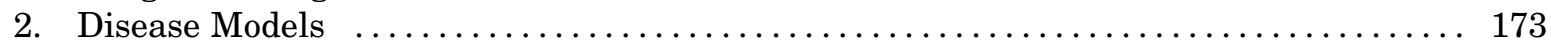

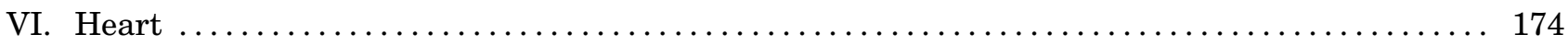

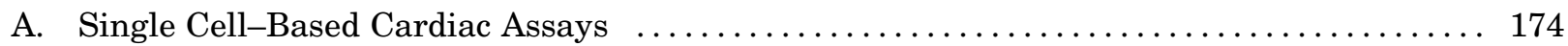

1. Electrophysiology Assays for Single Cardiomyocytes $\ldots \ldots \ldots \ldots \ldots \ldots \ldots \ldots \ldots \ldots$

2. Excitation-Contraction Coupling in Single Cardiomyocytes $\ldots \ldots \ldots \ldots \ldots \ldots \ldots \ldots \ldots$

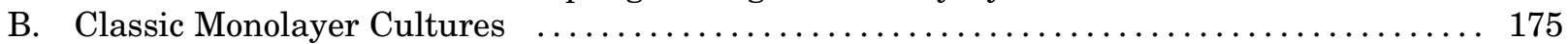

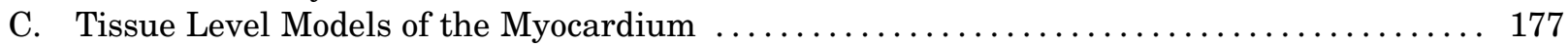

1. Cardiac Cell Aggregates as Scaffold-Free 3D Models $\ldots \ldots \ldots \ldots \ldots \ldots \ldots \ldots \ldots \ldots . \ldots \ldots$

2. Scaffold-Based Cardiac Tissue Models $\ldots \ldots \ldots \ldots \ldots \ldots \ldots \ldots \ldots \ldots \ldots \ldots \ldots \ldots \ldots$

D. Applications of Organotypic Models of the Heart $\ldots \ldots \ldots \ldots \ldots \ldots \ldots \ldots \ldots \ldots \ldots \ldots$

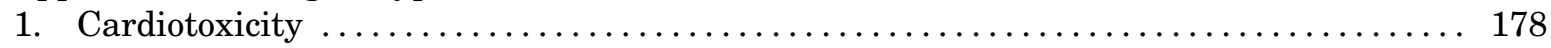

2. Cardiac Disease Models for Drug Development $\ldots \ldots \ldots \ldots \ldots \ldots \ldots \ldots \ldots \ldots \ldots \ldots$

VII. Skeletal Muscle $\ldots \ldots \ldots \ldots \ldots \ldots \ldots \ldots \ldots \ldots \ldots \ldots \ldots \ldots \ldots \ldots \ldots \ldots \ldots \ldots \ldots \ldots \ldots . \ldots \ldots$

A. Primary Myoblast-Derived 3D Culture $\ldots \ldots \ldots \ldots \ldots \ldots \ldots \ldots \ldots \ldots \ldots \ldots \ldots \ldots \ldots$

B. Pluripotent Stem Cell-Derived Muscle Models $\ldots \ldots \ldots \ldots \ldots \ldots \ldots \ldots \ldots \ldots \ldots \ldots \ldots 182$

C. Applications of 3D Human Skeletal Muscle Models $\ldots \ldots \ldots \ldots \ldots \ldots \ldots \ldots \ldots \ldots \ldots \ldots 182$

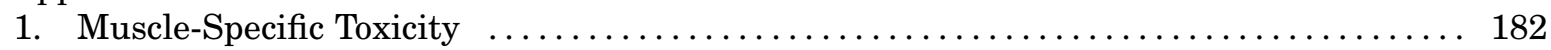

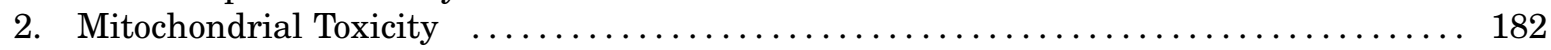

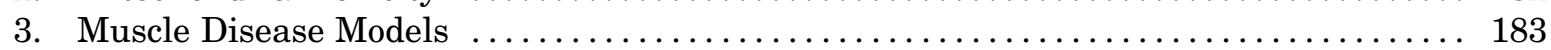

VIII. Fluidic Integration of Organotypic Tissue Models $\ldots \ldots \ldots \ldots \ldots \ldots \ldots \ldots \ldots \ldots \ldots \ldots \ldots \ldots . \ldots \ldots$

IX. Bioprinting as a Novel Modality for the Generation of Organotypic Cultures ............ 186

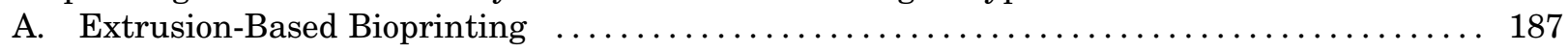

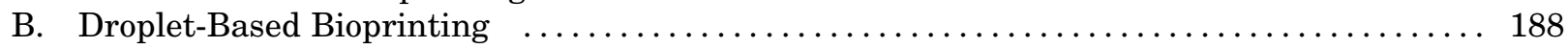

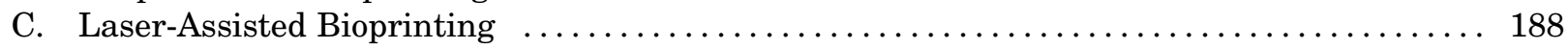

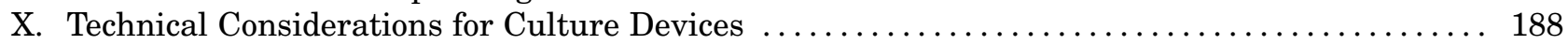

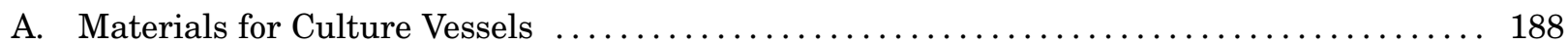

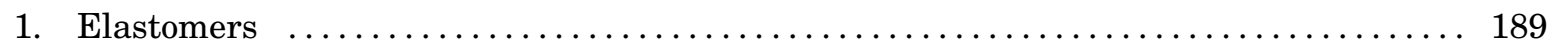

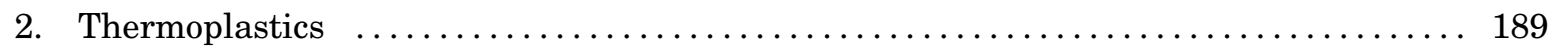

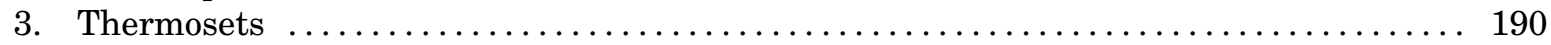

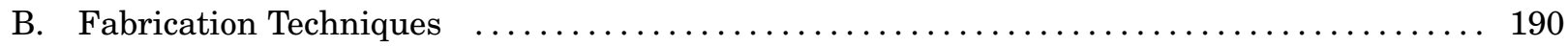

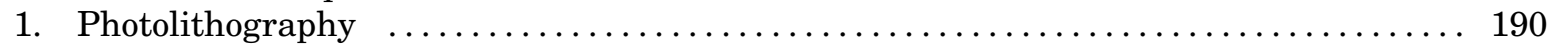

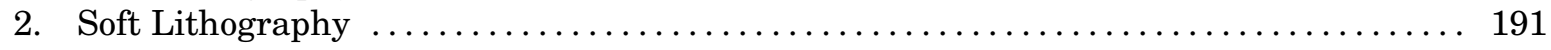

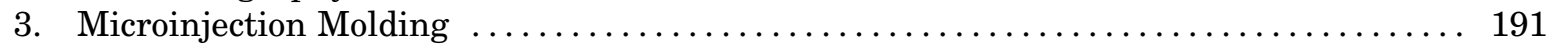

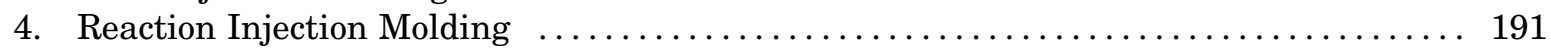

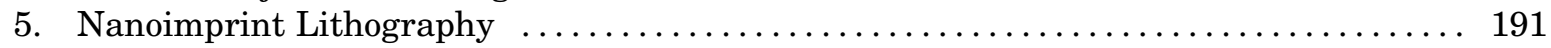

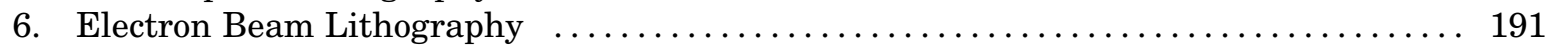

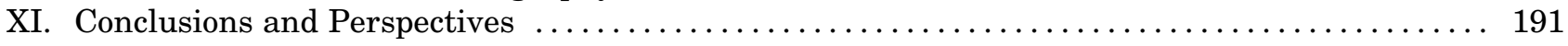

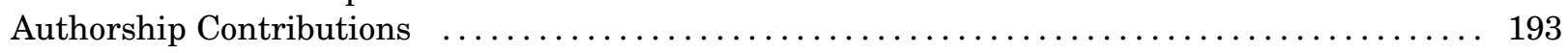

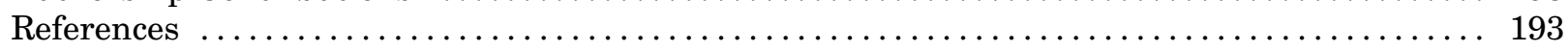

\begin{abstract}
The number of successful drug development projects has been stagnant for decades despite major breakthroughs in chemistry, molecular biology, and genetics. Unreliable target identification and poor translatability of preclinical models have been identified as major causes of failure. To improve predictions of clinical efficacy and safety, interest has shifted to three-dimensional culture methods in which human cells can retain many physiologically and functionally relevant phenotypes for extended periods of time. Here, we review the state of the art of available organotypic culture techniques and critically review emerging models of human tissues with
\end{abstract}

key importance for pharmacokinetics, pharmacodynamics, and toxicity. In addition, developments in bioprinting and microfluidic multiorgan cultures to emulate systemic drug disposition are summarized. We close by highlighting important trends regarding the fabrication of organotypic culture platforms and the choice of platform material to limit drug absorption and polymer leaching while supporting the phenotypic maintenance of cultured cells and allowing for scalable device fabrication. We conclude that organotypic and microphysiological human tissue models constitute promising systems to promote drug discovery and development by facilitating drug 
target identification and improving the preclinical evaluation of drug toxicity and pharmacokinetics. There is, however, a critical need for further validation, benchmarking, and consolidation efforts ideally conducted in intersectoral multicenter settings to accelerate acceptance of these novel models as reliable tools for translational pharmacology and toxicology.
Significance Statement_-Organotypic and microphysiological culture of human cells has emerged as a promising tool for preclinical drug discovery and development that might be able to narrow the translation gap. This review discusses recent technological and methodological advancements and the use of these systems for hit discovery and the evaluation of toxicity, clearance, and absorption of lead compounds.

\section{Introduction}

Discovery and development of safe and effective medicines critically depends on the accurate translation of robust preclinical findings. To this end, a multitude of enabling technologies have been developed, including combinatorial chemistry that allows for more efficient generation of chemically diverse screening libraries, omics technologies that provide accurate and comprehensive phenotypic profiling data, and gene editing tools, which enable the rapid generation of mechanistically relevant in vitro and in vivo models. However, despite these advancements, the number of successful development projects has stagnated over the last decades (Mullard, 2021). Although current preclinical models successfully remove the bulk of ineffective or toxic compounds, the likelihood of approval of a drug entering clinical development remains low at around $10 \%$ (Hay et al., 2014). The underlying reasons are complex and multifaceted; however, the shallow mechanistic profiling of putative new targets and the limited validity of preclinical models are consistently identified as contributors to the overall low success rates (Janero, 2014; Van Norman, 2019).

Although outcomes of animal research remain pivotal for regulatory approval to enter clinical stages, the translation gap between animal and human data is becoming increasingly appreciated. For instance, of 76 highly cited animal studies published in leading scientific journals, only $37 \%$ were validated in humans, whereas $18 \%$ were tested, but randomized trial results were contradictory
(Hackam and Redelmeier, 2006). Furthermore, only three of six interventions with well established clinical effects across 221 studies had similar outcomes in animal models, whereas the other three did not (Perel et al., 2007). Similarly low predictive accuracy was obtained for safety predictions. Only $19 \%$ of 93 serious postmarketing adverse reactions related to 43 small-molecule drugs were identified in preclinical animal experiments (van Meer et al., 2012), and particularly, the absence of animal toxicity was a poor predictor of human safety (Bailey et al., 2014). In addition to the limited translation of efficacy and safety across species, the diversity of human populations constitutes a major challenge that is not captured using animal models.

Organotypic and microphysiological cell culture of human cells using spheroids, organoids, or microfluidic devices aspires to facilitate result translation, opening up exciting opportunities for drug discovery and development. In some of these systems, human cells can be cultured with relevant molecular phenotypes and functions for extended periods of time-often weeks to months-thus enabling the study of long-term human drug absorption, distribution, metabolism, and excretion (ADME) as well as of chronic toxicity. Three-dimensional (3D) culture can interface with gene editing methods and offers the possibility to mimic a multitude of metabolic, inflammatory, and congenital diseases, opening new possibilities for drug discovery in human models that accurately mimic the pathology of interest. Furthermore, with sufficient scalability, advanced human in vitro models might allow to parse human diversity by investigating

ABBREVIATIONS: ABC, ATP-binding cassette transporters; ADME, absorption, distribution, metabolism, and excretion; ALS, amyotrophic lateral sclerosis; AQP, aquaporin; AP, action potential; ASD, autism spectrum disorder; $\mathrm{AUC}_{\mathrm{ROC}}$, area under the receiver operating characteristic; $\mathrm{BBB}$, blood-brain barrier; $\mathrm{BCRP}$, breast cancer resistance protein; BSEP, bile salt export pump; $\mathrm{CL}_{\text {int }}$, intrinsic clearance; COP, cyclic olefin polymer; COVID-19, coronavirus disease 2019; 2D, two-dimensional; 3D, three-dimensional; DIKI, drug-induced kidney injury; DILI, drug-induced liver injury; DMD, Duchenne muscular dystrophy; DOD, drop-on-demand; DSS, dextran sodium sulphate; EB, embryoid body; EBL, electron beam lithography; ECM, extracellular matrix; EHT, engineered heart tissue; EMA, European Medicines Agency; ERG, Ether-à-go-go-related gene; ESC, embryonic stem cell; FDA, US Food and Drug Administration; GST, glutathione S-transferases; HBV, hepatitis B virus; hiPSC, human induced pluripotent stem cell; HLC, hepatocyte-like cell; HO-1, heme oxygenase-1; HSC, hepatic stellate cell; IBD, inflammatory bowel disease; IL, interleukin; $\mu \mathrm{IM}$, microinjection molding; IM, injection molding; iPSC, induced pluripotent stem cell; IRI, ischemia-reperfusion injury; ITOP, integrated tissue-organ printing; LAB, laser-assisted bioprinting; LGR5, Leucine-rich repeat-containing G-protein coupled receptor 5; LIFT, laser-induced forward transfer; LSEC, liver sinusoidal endothelial cell; MEA, microelectrode array; miR, microRNA; MOOC, multiorgan-on-a-chip; MPCC, micropatterned coculture; MRP, multidrug resistance protein; NAFLD, nonalcoholic fatty liver disease; NASH, nonalcoholic steatohepatitis; NGAL, neutrophil gelatinase-associated lipocalin; NIL, nanoimprint lithography; NMJ, neuromuscular junction; NPC, nonparenchymal liver cell; OAT, organic anion transporter; P450, cytochrome P450; PAH, para-aminohippurate; PAX, paired box gene; PC, polycarbonate; PDMS, poly(dimethyl siloxane); PEG, poly(ethylene glycol); PHH, primary human hepatocyte; PMMA, polymethyl methacrylate; PS, polystyrene; PTEC, proximal tubule epithelial cell; RIM, reaction injection molding; ROS, reactive oxygen species; SARS-CoV-2, severe acute respiratory syndrome coronavirus 2; SGLT, sodiumglucose transporter protein; SLC, solute carrier; $\alpha$ SMA, stellate cell activation; TEER, transepithelial electrical resistance; UGT, UDP-glucuronosyltransferase; ULA, ultra-low attachment; WT1, Wilm's tumor protein 1; ZIKV, Zika virus. 
different human subpopulations before entering clinical trials.

Importantly, however, the added translational value of advanced human models must be balanced against their cost, throughput, and speed to justify their implementation into drug discovery and development pipelines. This is particularly relevant for models that aim to capture human variability and thus use material from different donors, as those platforms generally require a considerable number of experimental replicates to achieve statistically significant results. Here, we summarize recent progress in the organotypic culture of human tissues with major importance for drug disposition and discuss their advantages and limitations. Furthermore, we emphasize considerations regarding the choice of platform materials and highlight emerging technological trends, such as bioprinting and the microfluidic integration of different tissue models for studies of tissue interactions and systemic drug response predictions.

\section{Liver}

The liver is of central importance for drug pharmacokinetics and toxicology, and evaluation of the interactions between liver and drug candidates constitutes a key step in preclinical drug development. Importantly, hepatic functions differ both quantitatively and qualitatively across species with regards to expression patterns, isoform composition, and substrate specificity of enzymes and transporters involved in drug ADME (Martignoni et al., 2006; Chu et al., 2013). As a consequence, human in vitro or ex vivo liver models are used to complement preclinical animal data and enhance safety assessments in drug discovery (Olson et al., 2000; Hughes, 2008; Shanks et al., 2009). From the perspective of drug developers, it is of particular importance that liver cells in the culture system of choice are functional (albumin production and urea synthesis as well as relevant expression of drug-metabolizing enzymes and transporters) and that biomarkers and metabolic activity remain stable over time (Baudy et al., 2020). Furthermore, the model should have been benchmarked using a standardized set of mechanistically distinct hepatotoxic and nontoxic training compounds that allow for comparisons with other liver systems.

\section{A. Critical Overview of Available Hepatic Cell Models}

To emulate human liver function, a variety of cell models is available, including cancer-derived cell lines, induced-pluripotent stem cell (iPSC)-derived hepatocyte-like cells (HLCs) and primary human hepatocytes (PHHs). The hepatoma cell line HepG2 constitutes a commonly used cell model to study hepatotoxicity. These cells are readily available, immortalized, and easy to use. However, they are highly dedifferentiated and express only very low levels of drug-metabolizing enzymes, and their molecular phenotypes do not closely resemble human hepatocytes. HepaRG cells provide a promising alternative. Established from a liver tumor associated with chronic hepatitis $\mathrm{C}$, this cell line is bipotent in culture and can be differentiated into both biliary-like and hepatocyte-like cells (Parent et al., 2004). After differentiation, these cells are functionally polarized and have considerably higher expression of $\mathrm{P} 450 \mathrm{~s}$ and phase II enzymes with exception of sulfotransferases as well as drug transporters compared with HepG2 cells, rendering them an attractive cell model for toxicity studies and pharmacokinetic analyses (Hart et al., 2010; Gerets et al., 2012; Yokoyama et al., 2018; Gupta et al., 2021). However, although their metabolic activity outperforms HepG2 cells, overall P450 levels in HepaRG cells remain approximately $60 \%$ lower than in PHH (compared with 90\% lower in HepG2) (SisonYoung et al., 2015; Nelson et al., 2017), and, in a multicenter trial, both hepatoma cell models were found to be considerably less sensitive to a panel of nine hepatotoxins than PHH (Sison-Young et al., 2017).

Differentiation of iPSCs or embryonic stem cells (ESCs) into HLCs constitutes an additional strategy that has gained traction in recent years. These cells offer the advantage that they are not immortalized and genetically stable. Various protocols for HLC differentiation have been established (Si-Tayeb et al., 2010; Hannan et al., 2013; Du et al., 2014, 2018). However, although differentiated cells display important hepatic functions, such as albumin and urea secretion, and P450 activity as well as glycogen storage, no terminal differentiation has yet been achieved, and the molecular phenotypes of HLCs remain closer to fetal than adult hepatocytes (Goldring et al., 2017).

As a consequence, $\mathrm{PHH}$ remain the gold standard cell model for liver cell studies, as they express physiologic levels of drug-metabolizing enzymes and transporters (Gómez-Lechón et al., 2014). Furthermore, $\mathrm{PHHs}$ recapitulate the interindividual differences and patient-specific factors, such as genetic predispositions or comorbidities, thus allowing the study of gene-drug and disease-drug interactions in specific patient populations that are difficult to assess in clinical stages (Lauschke and IngelmanSundberg, 2016).

PHHs can be isolated using established two-step perfusion protocols, primarily using resected liver tissue (Bhogal et al., 2011; Lee et al., 2013b). Importantly, however, although they express physiologically relevant levels of drug-metabolizing enzymes and transporters at isolation, their molecular phenotype and function rapidly deteriorate in conventional monolayer culture. Specifically, transcriptomic and proteomic analyses showed that $\mathrm{PHHs}$ dedifferentiate extraordinarily rapidly in two-dimensional (2D) culture with first changes being apparent as early as 30 minutes after plating and many ADME genes being 
downregulated $>90 \%$ within 24 hours in a process driven by noncoding RNA signatures inside the cells (Lauschke et al., 2016b; Heslop et al., 2017).

Mechanistically, human hepatocyte dedifferentiation is closely linked to the cellular microenvironment. Culture on stiff collagen activates focal adhesion kinase and extracellular signal-regulated kinase signaling and triggers epithelial-to-mesenchymal transition, whereas culture on a soft collagen gel does not activate focal adhesion kinase and epithelial-to-mesenchymal transition (Godoy et al., 2009). Additional work showed that dedifferentiation was caused by mechanical tensions due to cell spreading, which resulted in YAP activation (Sun et al., 2019). Physical confinement of cells or chemical inhibition of stress fiber formation inhibited dedifferentiation and contributed to long-term functional maintenance of hepatic gene expression signatures.

Further mechanistic insights were provided by two recent studies that presented small-molecule combinations for the partial inhibition of dedifferentiation in $2 \mathrm{D}$ culture. One strategy used an adenylate cyclase activator combined with inhibitors of Notch, TGF $\beta$, and BMP signaling and a blocker of Wnt secretion (Xiang et al., 2019), whereas the other strategy combined TGF $\beta$ inhibition with inhibitors or rho-associated protein kinase and mitogen-activated protein kinase as well as an activator of $\mathrm{Wnt} / \beta$-catenin signaling (Katsuda et al., 2020). Notably, although both approaches report strongly improved viability and hepatic functions for up to 40 days in monolayer culture, substantial alterations of gene expression levels compared with freshly isolated hepatocytes remain. Furthermore, recent work showed that sensitivity of chemically stabilized PHH monolayer cultures to hepatotoxins, such as chlorpromazine $\left[\mathrm{TC}_{50}\right.$ (concentration that causes $50 \%$ cell death) $>200 \mu \mathrm{M}$; $\mathrm{c}_{\max }$ (peak serum concentration) in vivo $=1.6 \mu \mathrm{M}]$ and acetaminophen $\left(\mathrm{TC}_{50}>15 \mathrm{mM}\right.$; $\mathrm{c}_{\max }$ in vivo in patients with acetaminophen-induced acute liver failure $=0.7-1.3$ $\mathrm{mM}$ ), remains orders of magnitude higher than patients in vivo (Chen et al., 2021). Combined, these data suggest that a complex signaling network involving biochemical and mechanosensitive cues underlies dedifferentiation of $\mathrm{PHHs}$ in 2D cultures. To delay or prevent dedifferentiation, a multitude of more advanced liver culture models have been developed that use different strategies to more accurately mimic the organotypic microenvironment (Lauschke et al., 2016a, 2019; Lin and Khetani, 2016; Zhang et al., 2020).

\section{B. Sandwich Culture}

In human liver, hepatocytes are polarized with apical and basal domains that are segregated by tight junctions. The apical membrane lies between the hepatocytes, whereas the basal membrane faces the lumen and interacts directly with the extracellular matrix (ECM). Evidence showed that ECM not only functions as a scaffold that ensures the structural integrity but also exerts biologic effects that modulate cell phenotype and behavior. To mimic this configuration in vitro, Dunn and colleagues (1989) first introduced the so-called sandwich culture configuration in 1989, in which rat hepatocytes were cultured between two layers of collagen gel. They observed that in this model hepatocytes retained polygonal morphology and the secretion of albumin, transferrin, fibrinogen, and bile acids. Furthermore, the ECM overlay significantly contributed to in vivo-like hepatocyte polarization (Berthiaume et al., 1996). Like rat hepatocytes, also PHH sandwich culture maintained polygonal morphology with bile canaliculus-like structures as well as albumin secretion and key P450 enzyme expression (Kern et al., 1997). Notably, activity of CYP1A2 and CYP3A of PHH remained stable over 5 days of sandwich culture, whereas CYP2C8, CYP2C9, CYP2C19, and CYP2D6 substantially decreased already after 24 hours (Kimoto et al., 2012). As such, the application of a matrix overlay substantially increases the functional lifetime of primary hepatocyte cultures compared with conventional monolayers.

In sandwich cultures, cells are typically plated on collagen with a matrigel overlay (Fig. 1A). In this configuration, sandwiched hepatocytes exhibit increased P450 activity and higher CYP3A inducibility compared with the respective single-layer cultures (Mingoia et al., 2007). In addition to collagen and matrigel overlays, heparin-containing hydrogels or porcine liver-derived ECM were presented as scaffolds for hepatocyte cultivation, and it was shown that they increased albumin secretion compared with conventional collagen gels (Sellaro et al., 2010; Foster et al., 2015). Whether similar effects can be observed in human hepatocytes remains to be determined.

A key strength of sandwich cultures is their formation of an intact bile canaliculi network, which opens possibilities to study biliary transport and excretion. Hepatocytes maintain bile acid homeostasis (Marion et al., 2012). Furthermore, expression of the hepatobiliary transporters BCRP and BSEP (bile salt export pump) in sandwich culture remained relatively stable ( $<2$-fold variability) throughout culture for 8 days as evaluated by liquid chromatography coupled with tandem mass spectrometry ( $\mathrm{Li}$ et al., 2009) (Fig. 1B). Importantly, biliary excretion of bile acids and xenobiotics can be measured by using buffers that disrupt tight junctions, resulting in the release of secreted bile into medium (Liu et al., 1999b; Bi et al., 2006), and data from rats indicate that in vitro biliary excretion rates correlate well with in vivo values (Liu et al., 1999a). In addition, biliary excretion can be evaluated using live cell imaging approaches in which canalicular dilation 
A

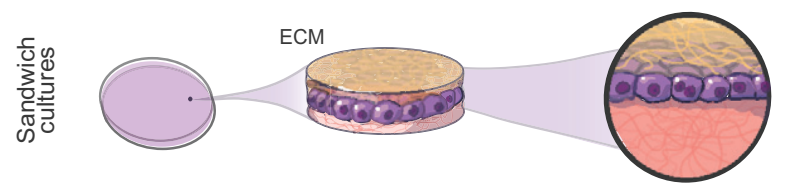

C

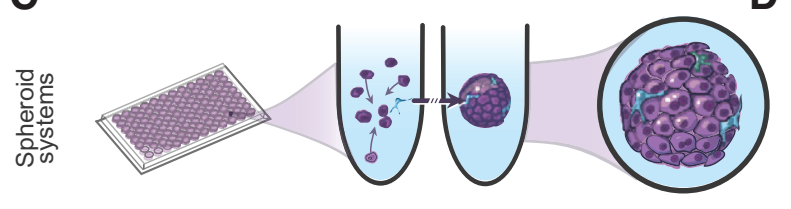

E

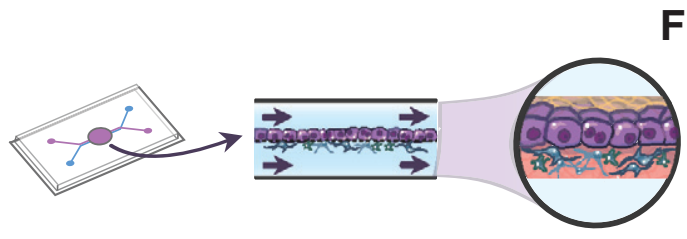

G

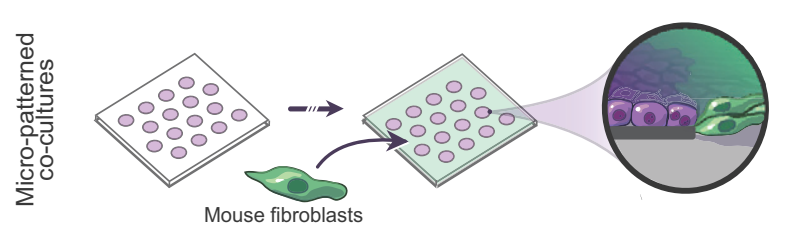

$\mathbf{F}$

H
B

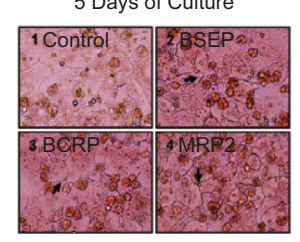

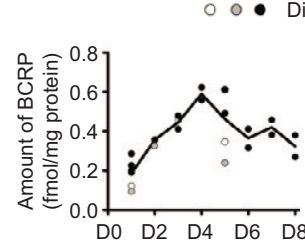

- Different donors

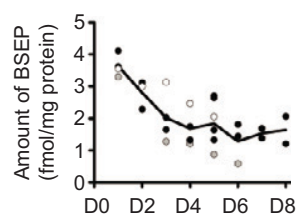

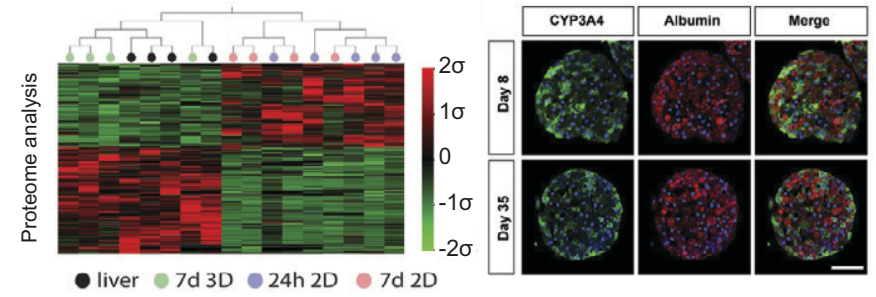
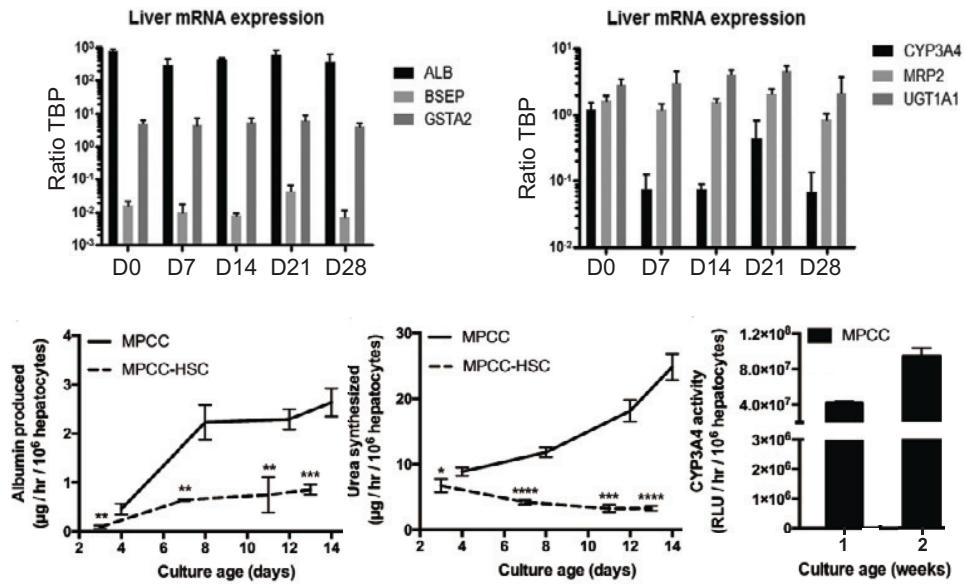

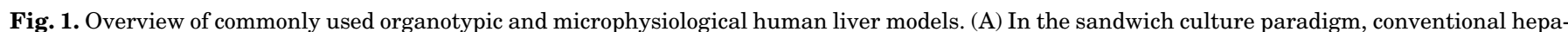

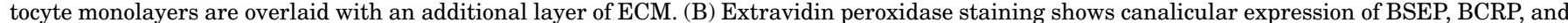

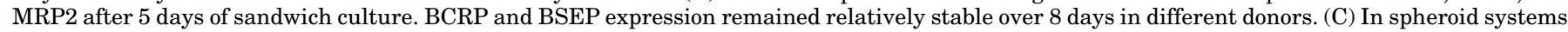

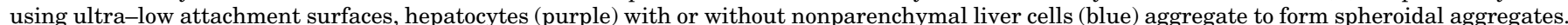

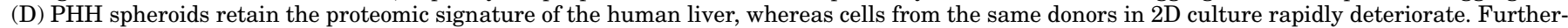

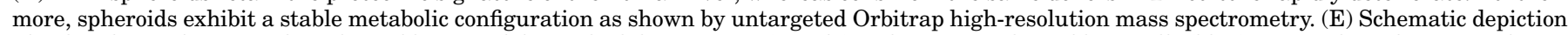

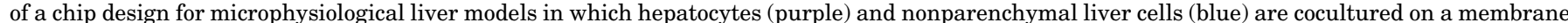

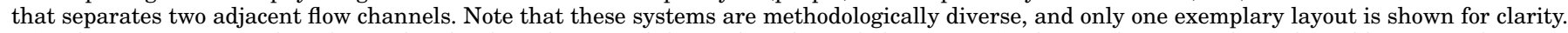

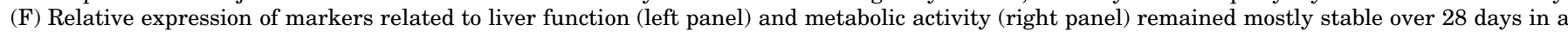

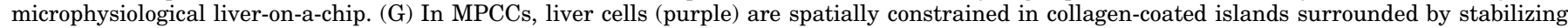

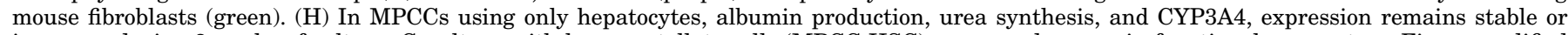

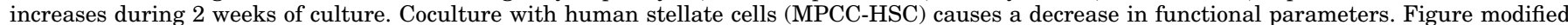
with permission from (Bell et al., 2016; Lauschke et al., 2016a; Li et al., 2009; Maschmeyer et al., 2015; Davidson et al., 2017).

and constriction dynamics are measured (Reif et al., 2015; Burbank et al., 2016). Currently, sandwich cultures are most commonly used for the quantitative evaluation of hepatobiliary transport and screening for the cholestatic liability of drugs and drug candidates.

\section{Spheroid Culture}

As an alternative to sandwich cultures, hepatocytes can be cultured as three-dimensional (3D) spheroidal aggregates by seeding cell suspensions into hanging drops or into U-shaped wells with ultra-low attachment (ULA) surfaces (Fig. 1C). These methods were first presented in the mid-1980s when rat hepatocyte spheroids formed on poly(2-hydroxyethyl methacrylate) or proteoglycancoated ULA surfaces and were shown to maintain viability for several weeks with hepatic functions superior to monolayer culture (Landry et al., 1985; Koide et al.,
1989; Tong et al., 1992). However, despite these promising results, the field remained dormant, and spheroid culture emerged as a widely used paradigm only more than 2 decades later (Ingelman-Sundberg and Lauschke, 2021).

Spheroid culture is compatible with the culture of HepG2, Huh-7, HepaRG, hiPSC-derived HLCs and PHHs and has been shown to contribute to improved phenotypes irrespective of cell models. In HepG2 spheroids, spheroid culture increased albumin secretion as well as CYP1A gene expression and inducibility (Shah et al., 2018). Similarly, HepaRG spheroids show increased P450 expression as well as albumin and lipoprotein secretion and maintain CYP3A-dependent metabolism and inducibility (Murayama et al., 2015; Takahashi et al., 2015). Furthermore, spheroid culture resulted in increased 
expression of CYP1A2 and CYP3A4 and lower levels of AFP in iPSC-derived HLCs; expression levels remained orders of magnitude lower than in $\mathrm{PHHs}$ (Meier et al., 2017).

For PHHs, spheroid culture drastically extends the lifetime of the cultured cells compared with monolayer or sandwich culture. Specifically, studies showed that $\mathrm{PHH}$ spheroids maintained stable transcriptomic, proteomic, and metabolomic signatures that closely resemble freshly isolated cells for at least 5 weeks, whereas cells from the same donors cultured as monolayers rapidly deteriorate (Bell et al., 2016; Vorrink et al., 2017; Messner et al., 2018) (Fig. 1D). Interestingly, during spheroid aggregation, $\mathrm{PHH}$ transiently dedifferentiate and redifferentiate once spheroids are formed (Oliva-Vilarnau et al., 2020). These molecular changes closely resemble the transcriptomic and proteomic alterations that occur after partial hepatectomy, and the model has been successfully used to identify Wnt-signaling mediated repression of p53 as a critical component of human liver regeneration.

Once spheroids are formed, activity of drug-metabolizing enzymes, such as CYP2C9, CYP2C19, CYP2D6, CYP3A4, UDP-glucuronosyltransferase (UGT) 2B7, sulfotransferase (SULT) 1A1, and GSTP1, were maintained, whereas CYP2E1 substantially decreased (Ohkura et al., 2014). Transcriptomic and functional comparison with other hepatic cells, such as iPSCderived HLCs and HepaRG cells, showed that expression of metabolic pathway genes was strongly increased in PHH spheroids $\left(P=3^{*} 10^{-33}\right)$ (Bell et al., 2017). Furthermore, direct benchmarking of PHH spheroids with sandwich culture in a multicenter trial using standardized protocols, cells, and reagents showed that expression of P450s and glutathione S-transferases (GSTs) as well as P450 activities were overall increased, whereas levels of major drug transporters (MDR1, OATP1B1, OCT1, and BSEP) were not significantly different (Bell et al., 2018).

Besides formation in ULA plates and hanging drops, PHH spheroids have been generated in automated, perfusion-stirred bioreactors, in which hepatocyte spheroids form over the course of 72 hours and maintain $70 \%$ cell viability and albumin and urea secretion as well as expression of CYP1A2, CYP2C9, CYP3A4, GSTA1, and UGT2B7 for 2 weeks (Tostões et al., 2012). Compared with multiwell static culture systems, the bioreactor tank ensures the consistent cultural environment for all spheroids, including oxygen supply, $\mathrm{pH}$, and culture medium composition. Particularly, the stirring system introduced high concentration of dissolved oxygen, which contributes to enhanced hepatic functions in various in vitro culture models (Kidambi et al., 2009). This culture paradigm was further modified by coculturing $\mathrm{PHH}$ with human bone marrow mesenchymal stem cells, which resulted in slightly enhanced expression of CYP1A2, CYP2C9, CYP3A4, and UGT1A1 (Rebelo et al., 2017). Although spheroid formation in stirred bioreactors is more rapid than in ULA plates or hanging drops (72 hours instead of 5-7 days), this method results in the formation of spheroids of heterogeneous sizes, which can increase interexperimental variability. Moreover, in this paradigm, spheroids are cultured in one or few compartments, which renders this method unable to study a large number of different conditions.

Liver spheroids can be cocultured with nonparenchymal liver cells (NPCs), including Kupffer, stellate, and endothelial cells. Such coculture was shown to enhance hepatic functions, as indicated by increased expression of albumin, apolipoprotein B, and CYP3A4 compared with $\mathrm{PHH}$ monoculture spheroid (Baze et al., 2018) and allows mimicking pathologies that involve a dysfunctional interplay between cell types, such as nonalcoholic steatohepatitis (NASH) and fibrosis.

Besides the scaffold-free methods described above, hepatic spheroids can be generated and maintained on a variety of synthetic or natural supports. Commonly used scaffolds for liver cell spheroid culture include alginate, cellulose, poly(ethylene glycol) (PEG), poly(styrene-co-maleic acid), and poly(L-lactic acid). Alginate scaffolds have been reported to increase hepatic functions in HepG2 and HepaRG cells (Elkayam et al., 2006; Rebelo et al., 2015) as well as in rat hepatocyte spheroids (Chan et al., 2016), whereas macroporous cellulosic scaffolds have shown beneficial effects for iPSC-derived HLCs (Tasnim et al., 2016). Similarly, PEG encapsulation resulted in increased expression of CYP3A4 and HNF4A in Huh-7.5 spheroids (Kim et al., 2016b). Although these polymers are biocompatible and provide defined chemical composition as well as tunable mechanical properties, they do not resemble the native biochemical microenvironment of the human liver. To overcome these limitations, human liver cell spheroids have been cultured on decellularized rat liver scaffolds, resulting in increased expression of phase I, phase II enzymes as well as drug transporters in HepaRG cells for up to 28 days (Liu et al., 2018a). Although these scaffolds provide a more relevant ECM than alginate or synthetic polymers, they are difficult to obtain from human sources, are not chemically characterized, and have substantial batch-to-batch variability. Furthermore, no clear benefits of scaffolds have been shown for PHH spheroids, and, thus, the use of such supports remains limited to hepatic cell lines and rodent cells.

\section{Microphysiological Liver Systems}

Although sandwich cultures and spheroids described above are cultured in static conditions, microphysiological liver systems introduce perfusion, which facilitates constant supply of oxygen and nutrients and removal 
of cellular waste products as well as physiologic shear stress and hemodynamics (Fig. 1E). As such, medium flow can emulate zonal specification of hepatocyte phenotypes, driven primarily by differences in oxygen tension (Kietzmann, 2017), and support the long-term maintenance of hepatic phenotypes (Fig. 1F). However, as hepatocytes are not in direct contact with the circulating blood in vivo, such microphysiological liver systems often integrate a barrier between the cells and the perfused medium in form of either physical barrier (Li et al., 2018a; Foster et al., 2019; Peel et al., 2019) or phaseguides, wherein the barrier effect is based on the meniscus-pinning effect (Vulto et al., 2011; Trietsch et al., 2013).

The fluidic devices for microphysiological liver models can be fabricated from a range of materials and we refer the interested reader to section $X$. Technical Considerations for Culture Devices of this review for an in-depth discussion of their specific advantages and limitations. One commonly used design consists of two parallel channels, one being loaded with hepatocytes and one with endothelial cells, separated by a porous pol$\mathrm{y}$ (dimethyl siloxane) (PDMS) membrane (Foster et al., 2019; Peel et al., 2019). In this configuration, activity of CYP1A2, CYP2D6, CYP2C9, CYP3A4, CYP2C19, and CYP2E1 could be maintained for 10 days of culture, and the metabolic profile of acetaminophen that resulted from the chip was comparable to profiles of human in vivo studies (Foster et al., 2019). Interestingly, metabolic activity of CYP1A2, CYP2C9, CYP2D6, and CYP3A4 was 10- to 100-fold lower than in spheroids, whereas CYP2E1 activity was 10 -fold increased. Using a PDMS device with similar layout consisting of murine hepatocytes and NPCs, it was furthermore demonstrated that perfusion significantly increased CYP1A2 and CYP2D6 activity by 5- to 10-fold (Du et al., 2017), thus demonstrating that flow is required for appropriate phenotypic maintenance of hepatic phenotypes of membrane cultures.

Other commonly used designs comprise bioreactor chambers with integrated pneumatic micropumps, inlet and outlet valves, and fluidic capacitors that convert pulsatile flow generated by the pump to constant flow (Domansky et al., 2010). In this device termed LiverChip, PHHs formed bile canalicular-like structures and remained viable for multiple weeks (Vivares et al., 2015). However, expression of the phase I enzymes CYP2C8, CYP2C9, and CYP2D6; the drug transporters OATP1B3 and BSEP; and the nuclear hormone receptors CAR and PXR declined considerably over culturing time. The platform has been shown to be compatible with the coculture of PHH and Kupffer cells and could mimic IL6-mediated suppression of CYP3A4 activity, which could be prevented using the anti-IL6 receptor antibody tocilizumab (Long et al., 2016).
In a separate microfluidic, sequentially layered PDMS-based liver chip (SQL-SAL), a hepatic chamber with PHH and Kupffer and stellate cell lines (U937 and LX-2, respectively) was separated by a porous membrane from a vascular channel containing the endothelial cell lines EA.hy926 (Vernetti et al., 2016). Notably, the device could emulate zonal, oxygendriven liver sinusoidal features and leukocyte infiltration, and the coculture remained viable for at least 4 weeks. As with other perfusion chips, functionality (albumin) was maintained for at least 4 weeks and remained higher than in static cultures. The device was further refined by using primary human endothelial cells and decellularized liver ECM to mimic the space of Disse (Lee-Montiel et al., 2017). Furthermore, PDMS was replaced with glass to reduce drug absorption, and separate flow channels were introduced, which separate the sinusoidal and hepatic compartment ( $\mathrm{Li}$ et al., 2018a). The device allows the study of oxygen gradients on compounds' pharmacology and toxicity and facilitates investigations of leukocyte transmigration.

To increase the throughput of microphysiological liver systems, multiple groups have developed solutions that allow researchers to run tens to hundreds of perfused liver cultures in parallel. In a microfluidic system with 96-well layout made of thermoplastic materials (cyclic olefin copolymer), $\mathrm{PHH}$ are seeded in sandwich configuration and exhibit significantly increased expression of albumin, A1AT, VEGF, and HGF and show increased CYP3A4 activity compared with static conditions (Tan et al., 2019). Similarly, a chip with 40 culture chambers, each of which consists of three lanes separated by phaseguides, allows the culture of liver cells in a passively perfused environment in which flow is based on a gravity using a rocking device without the need for external micropumps (Jang et al., 2015; Jang et al., 2019b). In this device HepG2 and HepaRG cells can be cultivated for multiple weeks and show strongly increased albumin production and urea synthesis as well as a slight increase in the sensitivity to acetaminophen. Similarly, perfusion was shown to increase activity of CYP2B6, CYP2D6, CYP3A4, CYP2C19, CYP1A2, CYP2C8, CYP2C9, and phase II enzymes for multiple days of culture in polystyrene chips in which PHHs and NPCs were seeded on conventional rat tail collagen (Novik et al., 2010).

Notably, microphysiological liver systems can furthermore be combined with 3D spheroid culture. HepG2 cell spheroids are formed in a PDMS-based microwell layer covered by a transwell insert and a microfluidic channel layer (Ma et al., 2018). This setup allows the perfused culture of up to 1080 spheroids with low shear stress in an easy-to-assemble and easy-to-disassemble biomimetic environment. 
Spheroids showed increased albumin and urea synthesis compared with static $2 \mathrm{D}$ culture as well as elevated expression of phase I and phase II enzymes, hepatic nuclear receptors, and drug transporters. Furthermore, a culture chip for hepatocyte spheroids has been presented in which cells were constrained between a cover glass and a porous-ultrathin Parylene $\mathrm{C}$ membrane (Yu et al., 2017). In this setup, rat hepatocytes exhibited moderately increased function compared with unperfused spheroids and sandwich culture. Interestingly, the authors observe toxicity of acetaminophen in this system at concentrations of $1 \mathrm{mM}$, which constitutes relevant human overdose levels, even though rats are protected from acetaminophen-induced liver injury in vivo (McGill et al., 2012).

Using a chip with a perfused micropillar array allows for the in situ differentiation of dozens of iPSC aggregates into HLCs and improves their functionality, as indicated by increased albumin and urea secretion as well as elevated CYP3A4, CYP2C9, and CYP2B6 expression compared with static conditions (Wang et al., 2018a). Similarly, using a chip with Ctrap pillar structure allowed for the capture of $\mathrm{PHH}$ or iPSC-derived HLCs and allowed for the culture of the resulting spheroidal structures in perfused conditions for multiple weeks (Schepers et al., 2016).

Other perfusion systems use the packaging of liver cells into fibrous structures, which can then be perfused. Specifically, this approach has been demonstrated for the culture of HepG2 cells that are encapsulated into hydrogel microfibers, which were subsequently bundled and densely packaged into a perfusion chamber (Yajima et al., 2018). These microfibers could furthermore be coated with bovine carotid artery endothelial cells to resemble vascular networklike structures. Increase in flow rate induced expression of OTC, CYP1A2, and CYP3A4, whereas expression of CYP2C9 and albumin remained lower than in conventional 2D HepG2 culture (Yajima et al., 2018).

In summary, the field of microphysiological liver systems is highly heterogeneous with a multitude of methodologically distinct approaches. In these systems, perfusion improves hepatic differentiation and function and can establish sinusoidal zonation. However, many designs lack high-throughput compatibility, and the added value of the increase in complexity compared with static 3D cultures has not been demonstrated in comprehensive standardized benchmarking studies that directly compare model performance.

\section{E. Micropatterned Cocultures}

In micropatterned cocultures (MPCCs), hepatic cells selectively adhere to collagenated islands, which are surrounded by mouse 3T3-J2 fibroblasts (Fig. 1G). The balance between homotypic and heterotypic interactions results in higher albumin and urea secretion compared with cocultures with random organization. MPCCs of PHH form bile canaliculi and maintain gene expression of phase I and phase II drug-metabolizing enzymes, nuclear hormone receptors, and drug transporters for at least 6 weeks of culture (Khetani and Bhatia, 2008). Furthermore, MPCCs allow the coculture with NPCs. Specifically, primary human liver sinusoidal endothelial cells (LSECs) remained viable and functional for at least 3 weeks in culture and stimulated PHH albumin secretion (Ware et al., 2017), whereas cocultures with human Kupffer cells allow for the quantification of effects of inflammatory triggers, such as lipopolysaccharide, on expression of drug-metabolizing enzymes, drug transporters, and acute-phase proteins (Nguyen et al., 2015). By contrast, MPCC coculture of PHHs and activated human stellate cells results in reduced albumin production and urea synthesis and decreased activities of CYP2A6, CYP3A4, and transporters [BSEP and multidrug resistance protein (MRP) 2] as well as higher levels of IL6 and C-reactive protein, resembling effects seen in NASH (Davidson et al., 2017) (Fig. 1H). Besides primary cells, MPCC supports the functionality of iPSC-derived hepatocytes when using an additional overlay of matrigel, resulting in a 5- to 10 -fold increase in albumin and urea secretion compared with conventional monolayer cultures over the course of 3 weeks (Berger et al., 2015).

\section{F. Main Applications of Organotypic Liver Models}

1. Hepatotoxicity. Drug-induced liver injury (DILI) constitutes the most common cause of acute liver failure, accounting for $52 \%$ of acute liver failure cases (Ostapowicz et al., 2002). The majority of cases are attributed to acute acetaminophen toxicity, whereas the remaining DILI events are caused by a multitude of different drugs, including antibiotics and nonsteroidal anti-inflammatory drugs, and manifest mostly after multiple days to months after the initial exposure. Events allotted to those drugs are individually rare with DILI risk estimates per medications ranging from 1 in 10,000 to 1 in 100,000 individuals (de Abajo et al., 2004; Bell and Chalasani, 2009; Björnsson, 2010). As a result, in the general population, annual DILI rates excluding acetaminophen are estimated between 1 in 5000 to 1 in 10,000 (Sgro et al., 2002; Bjornsson et al., 2013). In addition, hepatotoxicity is of major concern in drug development and remains a leading cause of drug failure in preclinical, clinical, and postmarketing stages (Watkins, 2011; Onakpoya et al., 2016). Importantly, however, the risk of failure due to safety is in strong anticorrelation with the confidence in the preclinical safety profile, indicating that thorough preclinical safety testing reduces the risk of safety-related project closures during expensive clinical stages (Cook et al., 2014). 
2D monolayer cultures of PHHs have long constituted the cornerstone of in vitro hepatotoxicity testing. However, these models are short-lived and thus can, at best, mimic acute toxicity. To capture the chronic and delayed toxicity associated with most DILI culprit drugs, long-term stable cultures are needed. Given that hepatotoxicity is often tested relatively early in the preclinical pipeline, systems should allow for a relatively high throughput. Consequently, mainly sandwich cultures, spheroids, and MPCCs have been tested and benchmarked for their ability to predict DILI using a large set of drugs. In the following, we provide an updated overview of toxicological screens conducted in 3D human liver models. For other applications, such as mechanistic investigations, comparative studies, or toxicogenomics, we refer the interested reader to recent comprehensive reviews (Zhou et al., 2019; Zhang et al., 2020; Lauschke, 2021; Serras et al., 2021).

Sandwich cultures have primarily been used for investigations of cholestatic hepatotoxicity. Cholestatic toxicity is evaluated by coexposure of cultures with bile acid mixtures (i.e., a compound is classified as cholestatic if bile acids amplify its toxicity). Based on this principle, sandwich cultures could distinguish the cholestatic compounds cyclosporin $\mathrm{A}$,
A

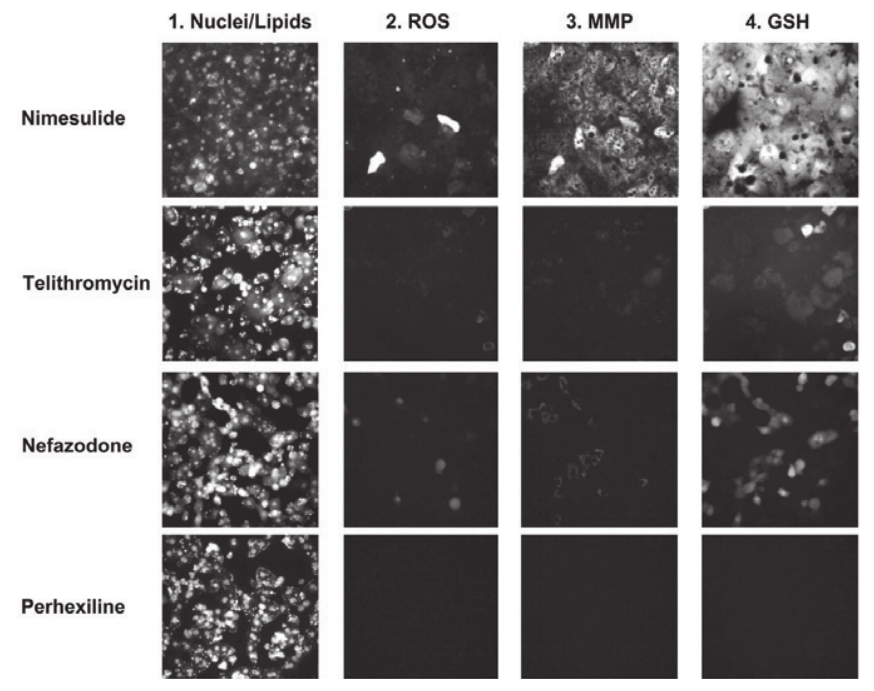

C

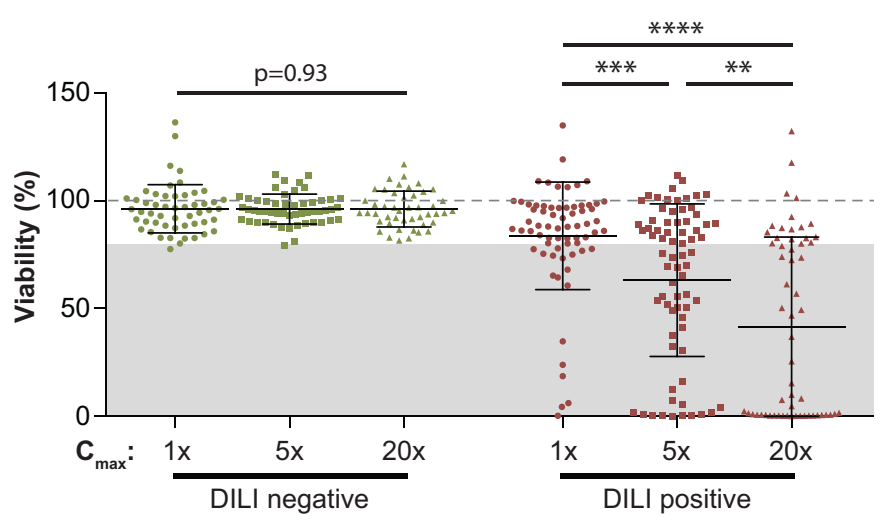

Liver-on-a-chip platforms

D $\quad \operatorname{APAP}(\mathrm{mM})$ :

0

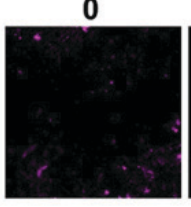

$\operatorname{MTX}(\mu \mathrm{M})$ :

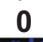

0

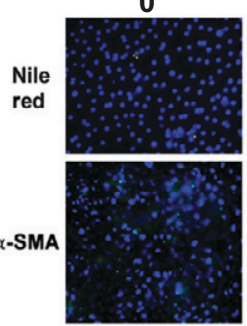

0.5

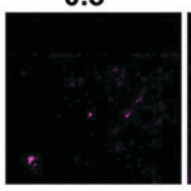

1

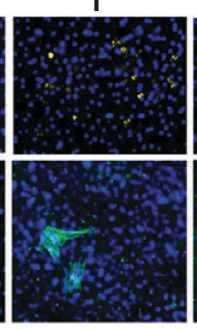

3

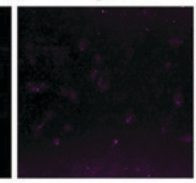

10

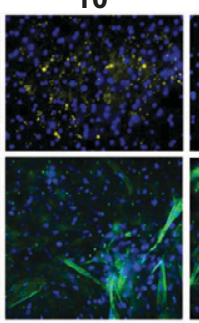

10

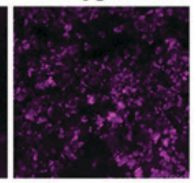

30

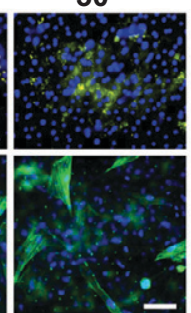

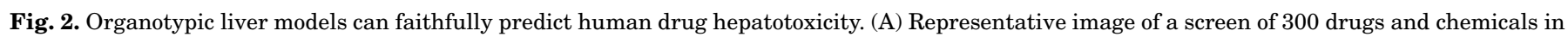

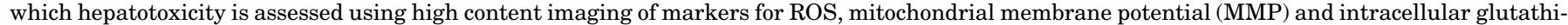

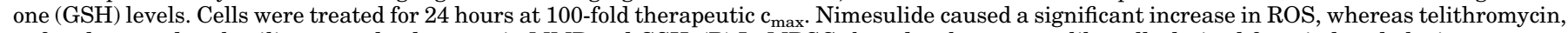

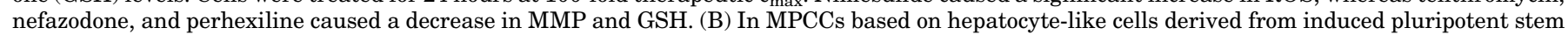

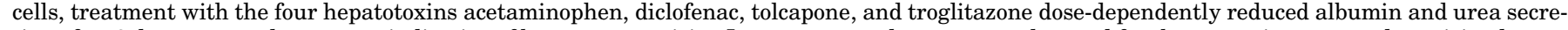

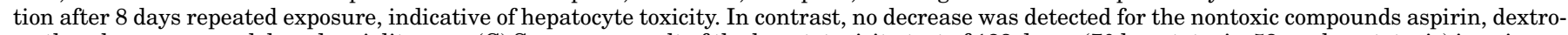

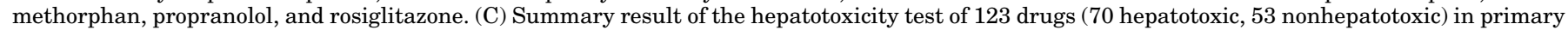

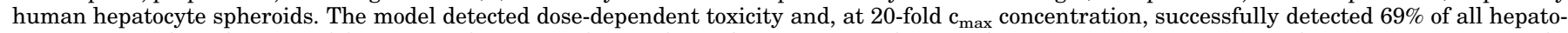

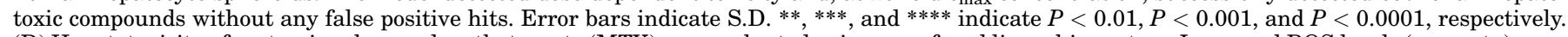

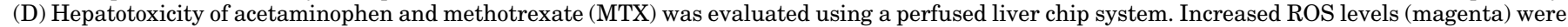

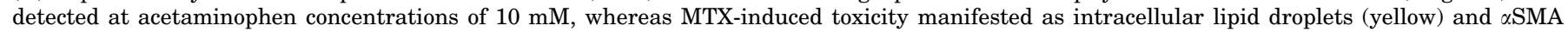
(green). Figure modified with permission from (Jang et al., 2019a; Berger et al., 2015; Vorrink et al., 2018; Xu et al., 2008). 
nefazodone, troglitazone, perhexillin, chlorpromazine, bosentan, ticlopidine, and ritonavir and from eight hepatotoxins that exert toxicity by different mechanisms and nontoxic controls (Marion et al., 2007; De Bruyn et al., 2013; Chatterjee et al., 2014; Oorts et al., 2016). Furthermore, sandwich cultures have been established for hepatotoxicity screening irrespective of toxicity mechanisms (Fig. 2A). Evaluation of the acute hepatotoxicity of 344 compounds (200 DILI positives and 144 DILI negatives; 24 hours exposure) in $2 \mathrm{D}$ sandwich cultures of $\mathrm{PHH}$ using automatic imaging for mitochondrial damage, oxidative stress, and intracellular glutathione resulted in the correct classification of all nontoxic drugs and the identification of 101 of 200 hepatotoxins (sensitivity $51 \%$, specificity $100 \%$ ) (Xu et al., 2008). Notably, however, these predictions relied on the calibration of the individual cutoffs, and an application of the system to further independent test sets has not been performed.

Evaluation of drug hepatotoxicity of 13 compounds after short-term exposure identified most toxic compounds apart from acetaminophen, whereas the nontoxic controls aspirin and caffeine were successfully identified as nontoxic (Khetani and Bhatia, 2008). Using a multiplexed toxicity assay comprising measurements of ATP, urea, albumin, and glutathione, exposure of MPCCs of $\mathrm{PHH}$ for 9 days successfully flagged 23 out of 35 compounds as hepatotoxic, whereas 9 out of 10 DILI negative compounds were identified as true negatives (sensitivity $66 \%$, specificity $90 \%$ ) when using the ratio of $\mathrm{IC}_{50}$ and total plasma $\mathrm{C}_{\max }$ for classification (Khetani et al., 2013). Changing the cell model to iPSC-derived HLCs, the four prototypical hepatotoxins acetaminophen, diclofenac, tolcapone, and troglitazone were successfully identified as hepatotoxic after 8 days exposure, whereas the nontoxic controls aspirin, dextromethorphan, propranolol, and rosiglitazone were flagged as nontoxic (Fig. 2B). Further extension of this model resulted in the identification of 24 out of 37 hepatotoxic drugs, whereas all 10 nontoxic drugs were correctly classified (sensitivity $65 \%$, specificity 100\%) (Ware et al., 2015).

Spheroids have emerged as the predominant $3 \mathrm{D}$ model for the prediction and mechanistic analysis of DILI. To analyze the hepatotoxic liability of compounds, most earlier studies used spheroids generated from cell lines, such as HepG2 (Mueller et al., 2011; Fey and Wrzesinski, 2012; Ramaiahgari et al., 2014; Gaskell et al., 2016) and HepaRG (Leite et al., 2012; Gunness et al., 2013; Mueller et al., 2014; Wang et al., 2015, 2016; Ott et al., 2017; Ramaiahgari et al., 2017; Liu et al., 2018a). However, these studies only evaluated small sets of compounds $(<20)$ and thus do not allow firm conclusion about their predictive accuracy. For spheroids generated from iPSC-derived HLCs, two smaller screens have been published that evaluated the toxicity using single-dose exposures. In a study of 24 compounds (20 DILI-positive and 4 DILI-negative), HLCs were found to perform better than HepG2 cells but remained less sensitive than $\mathrm{PHH}$ monolayer cultures (Takayama et al., 2013). In a separate study using 48 (42 DILI-positive and 6 DILI-negative) compounds, evidence of toxicity was identified in 36 of the toxic drugs, whereas the 6 nontoxic compounds remained negative (Sirenko et al., 2016). However, the choice of test compounds was problematic, as the DILI-positive set included various compounds with general cytotoxicity (anthracyclines, taxanes, alkaloids, and platinum compounds), whereas the negative set comprised carbohydrates (sucrose and sorbitol) and commonly used media supplements (penicillin and streptomycin).

For PHH spheroids two larger screens based on simple high-throughput compatible ATP endpoints were published that used compound sets based on regulatory classifications. Two weeks treatment of spheroids cultured in undisclosed media compositions at 100 times the therapeutic $\mathrm{C}_{\max }$ of 110 drugs (69 DILI-positive and 41 DILI-negative) resulted in a sensitivity of $59 \%$ and a specificity of $80 \%$ (Proctor et al., 2017). In contrast, spheroids cultured in conventional ULA plates in chemically defined conditions that were exposed for 2 weeks to 123 compounds (70 DILI-positive and 53 DILI-negative) at 20 times $\mathrm{C}_{\max }$ further improved the predictive accuracy to $69 \%$ sensitivity and $100 \%$ (Vorrink et al., 2018) (Fig. 2C). Notably, spheroids detected toxicity irrespective of DILI pattern and underlying toxicity mechanism, including mitochondrial toxicity and cholestatic toxicity (Hendriks et al., 2016; Hendriks et al., 2019).

Hepatotoxicity has furthermore been evaluated in cocultured spheroids of PHHs and NPCs wherein the latter appear to modulate compound toxicity, likely at least in part by impacting drug metabolism. For instance, NPC coculture resulted in lower expression of CYP2E1 and CYP3A4 and reduced glutathione depletion and acetaminophen hepatotoxicity (Bell et al., 2020). Interestingly, Kupffer cells appear to exert differential effects on compound toxicity. Although Kupffer cell cocultures corroborated the protective effects on acetaminophen, they amplified trovafloxacin toxicity $(\mathrm{Li}$ et al., 2020). Thus, these results suggest that liver spheroids can provide useful tools to investigate the effects of specific liver cell types or their subpopulations on hepatocyte function and drug toxicity.

Hepatotoxicity prediction using microphysiological liver systems is making progress, and proof-of-concept studies have demonstrated that such approaches can reveal drug effects on metabolic flux dynamics (Ehrlich et al., 2018) and identify compounds with a propensity for idiosyncratic DILI (Novik et al., 2017; Jang et al., 2019a) (Fig. 2D). However, at present, available small- 
TABLE 1

Comparison of the sensitivity of organotypic liver models

\begin{tabular}{|c|c|c|c|c|c|c|}
\hline Cell Model & Cell Type & Mono-/Coculture & Exposure Regimen & Exposure Time & $\mathrm{TC}_{50}$ & Reference \\
\hline \multicolumn{7}{|c|}{$\begin{array}{c}\text { Toxicity Mechanism: Reactive Metabolites } \\
\text { Acetaminophen }\left(\mathrm{C}_{\max }=0.136 \mathrm{mM} ; \mathrm{TC}_{50} \text { in } \mathrm{mM}\right)\end{array}$} \\
\hline Spheroid & $\mathrm{PHH}$ & Monoculture & Repeated & 14 days & $0.32-0.8$ & (Bell et al., 2018) \\
\hline Spheroid & $\mathrm{PHH}$ & Monoculture & Repeated & 14 days & 0.46 & (Bell et al., 2020) \\
\hline Spheroid & $\mathrm{PHH}$ & Coculture with NPCs & Repeated & 14 days & $\sim 0.5$ & (Richert et al., 2016) \\
\hline Spheroid & $\mathrm{PHH}$ & Coculture with NPCs & Repeated & 14 days & 0.57 & (Proctor et al., 2017) \\
\hline Spheroid & $\mathrm{PHH}$ & Coculture with Kupffer cells & Repeated & 14 days & $\sim 0.6$ & (Richert et al., 2016) \\
\hline Spheroid & $\mathrm{PHH}$ & Monoculture & Repeated & 14 days & 0.64 & (Bell et al., 2017) \\
\hline Spheroid & $\mathrm{PHH}$ & Coculture with NPCs & Repeated & 14 days & 0.75 & (Messner et al., 2013) \\
\hline Spheroid & $\mathrm{PHH}$ & Monoculture & Repeated & 14 days & 0.88 & (Hendriks et al., 2016) \\
\hline Spheroid & $\mathrm{PHH}$ & Monoculture & Single & $24 \mathrm{~h}$ & 0.93 & (Li et al., 2020) \\
\hline Spheroid & HepaRG & Monoculture & Repeated & 14 days & 1.1 & (Hendriks et al., 2016) \\
\hline Spheroid & HepaRG & Monoculture & Single & $24 \mathrm{~h}$ & $\sim 1.1$ & (Leite et al., 2012) \\
\hline Spheroid & $\mathrm{PHH}$ & Monoculture & Repeated & $96 \mathrm{~h}$ & 1.21 & (Bell et al., 2020) \\
\hline Spheroid & $\mathrm{PHH}$ & Monoculture & Repeated & 8 days & 1.3 & (Hendriks et al., 2016) \\
\hline Spheroid & $\mathrm{PHH}$ & Coculture with NPCs & Repeated & 14 days & 1.35 & (Bell et al., 2020) \\
\hline Spheroid & HepaRG & Monoculture & Repeated & 8 days & 1.8 & (Hendriks et al., 2016) \\
\hline Spheroid & $\mathrm{PHH}$ & Coculture with NPCs & Repeated & 10 days & 1.7 & (Foster et al., 2019) \\
\hline Spheroid & $\mathrm{PHH}$ & Coculture with Kupffer cells & Single & 5 days & 2.25 & (Li et al., 2020) \\
\hline Perfused liver-chip & $\mathrm{PHH}$ & Coculture with LSECs & Repeated & 10 days & 2.4 & (Foster et al., 2019) \\
\hline Spheroid & $\mathrm{PHH}$ & Coculture with NPCs & Repeated & $96 \mathrm{~h}$ & 2.46 & (Bell et al., 2020) \\
\hline Spheroid & HepaRG & Monoculture & Single & $24 \mathrm{~h}$ & 2.48 & (Wang et al., 2016) \\
\hline Spheroid & HepaRG & Monoculture & Single & $24 \mathrm{~h}$ & 2.7 & (Gunness et al., 2013) \\
\hline Spheroid & HepaRG & Monoculture & Repeated & 6 days & 2.879 & (Ramaiahgari et al., 2017) \\
\hline Spheroid & $\mathrm{PHH}$ & Monoculture & Single & 5 days & 3.28 & (Li et al., 2020) \\
\hline Spheroid & HepaRG & Monoculture & Repeated & 7 days & $\sim 4$ & (Liu et al., 2018a) \\
\hline MPCC & iPSC-HLC & Monoculture & Repeated & 6 days & 4.48 & (Ware et al., 2015) \\
\hline Perfused liver-chip & $\mathrm{PHH}$ & Coculture with NPCs & Repeated & 14 days & 5.59 & (Novik et al., 2017) \\
\hline Spheroid & HepG2 & Monoculture & Repeated & 4 days & 7.21 & (Gaskell et al., 2016) \\
\hline Spheroid & HepaRG & Monoculture & Single & $24 \mathrm{~h}$ & 7.62 & (Wang et al., 2015) \\
\hline Spheroid & HepG2 & Monoculture & Repeated & 6 days & $\sim 9.4$ & (Ramaiahgari et al., 2014) \\
\hline Spheroid & hiPSC/hESC-HLC & Monoculture & Single & $24 \mathrm{~h}$ & $>10$ & (Tasnim et al., 2016) \\
\hline Spheroid & hiPSC-HLC & Monoculture & Single & $24 \mathrm{~h}$ & $>20$ & (Takayama et al., 2013) \\
\hline Spheroid & HepG2 & Monoculture & Single & $24 \mathrm{~h}$ & $>20$ & (Takayama et al., 2013) \\
\hline MPCC & $\mathrm{PHH}$ & Monoculture & Single & $24 \mathrm{~h}$ & 35 & (Khetani and Bhatia, 2008) \\
\hline Spheroid & HepG2 & Monoculture & Single & $24 \mathrm{~h}$ & 40 & (Fey and Wrzesinski, 2012) \\
\hline
\end{tabular}

Toxicity Mechanism: Inhibition of Mitochondrial Respiration

Amiodarone $\left(\mathrm{C}_{\max }=5 \mu \mathrm{M} ; \mathrm{TC}_{50}\right.$ in $\left.\mu \mathrm{M}\right)$

\begin{tabular}{|c|c|c|c|c|c|c|}
\hline Spheroid & $\mathrm{PHH}$ & Monoculture & Repeated & 28 days & 1.6 & (Bell et al., 2016) \\
\hline Spheroid & $\mathrm{PHH}$ & Monoculture & Repeated & 14 days & 6.5 & (Bell et al., 2016) \\
\hline Perfused liver-chip & $\mathrm{PHH}$ & Coculture with NPCs & Repeated & 14 days & 9 & (Novik et al., 2017) \\
\hline Spheroid & $\mathrm{PHH}$ & Monoculture & Repeated & 14 days & 12 & (Bell et al., 2017) \\
\hline Spheroid & $\mathrm{PHH}$ & Monoculture & Repeated & 14 days & 12 & (Hendriks et al., 2016) \\
\hline MPCC & $\mathrm{PHH}$ & Monoculture & Repeated & 14 days & 14 & (Khetani et al., 2013) \\
\hline Spheroid & hiPSC-HLC & Monoculture & Single & $24 \mathrm{~h}$ & $>25$ & (Takayama et al., 2013) \\
\hline Spheroid & $\mathrm{PHH}$ & Monoculture & Single & 5 days & 26 & (Li et al., 2020) \\
\hline Spheroid & $\mathrm{PHH}$ & Coculture with NPCs & Repeated & 14 days & 32 & (Proctor et al., 2017) \\
\hline Spheroid & HepaRG & Monoculture & Single & 7 days & 49 & (Ott et al., 2017) \\
\hline Spheroid & HepG2 & Monoculture & Single & $24 \mathrm{~h}$ & $\sim 50$ & (Takayama et al., 2013) \\
\hline Spheroid & $\mathrm{PHH}$ & Coculture with NPCs & Repeated & 14 days & $\sim 60$ & (Richert et al., 2016) \\
\hline Spheroid & HepaRG & Monoculture & Single & $24 \mathrm{~h}$ & 83 & (Ott et al., 2017) \\
\hline Spheroid & HepaRG & Monoculture & Single & $24 \mathrm{~h}$ & 178 & (Mueller et al., 2014) \\
\hline Spheroid & HepG2 & Monoculture & Single & $24 \mathrm{~h}$ & $>200$ & (Mueller et al., 2014) \\
\hline Spheroid & HepG2 & Monoculture & Single & $24 \mathrm{~h}$ & 260 & (Fey and Wrzesinski, 2012) \\
\hline \multicolumn{7}{|c|}{$\begin{array}{c}\text { Toxicity Mechanism: Cholestasis } \\
\text { Chlorpromazine }\left(\mathrm{C}_{\max }=0.84 \mu \mathrm{M} ; \mathrm{TC}_{50} \text { in } \mu \mathrm{M}\right)\end{array}$} \\
\hline Spheroid & $\mathrm{PHH}$ & Monoculture & Single & 5 days & 4.4 & (Li et al., 2020) \\
\hline Spheroid & $\mathrm{PHH}$ & Monoculture & Repeated & 14 days & 4.6 & (Bell et al., 2017) \\
\hline Spheroid & $\mathrm{PHH}$ & Coculture with NPCs & Repeated & 14 days & $\sim 7$ & (Proctor et al., 2017) \\
\hline Spheroid & $\mathrm{PHH}$ & Coculture with only kupffer cells & Repeated & 14 days & $\sim 9$ & (Richert et al., 2016) \\
\hline Spheroid & $\mathrm{PHH}$ & Coculture with NPCs & Repeated & 14 days & $\sim 10$ & (Richert et al., 2016) \\
\hline Spheroid & $\mathrm{PHH}$ & Monoculture & Repeated & 14 days & 11 & (Hendriks et al., 2016) \\
\hline Spheroid & $\mathrm{PHH}$ & Monoculture & Repeated & 8 days & 13 & (Hendriks et al., 2016) \\
\hline Spheroid & HepaRG & Monoculture & Repeated & 14 days & 13 & (Hendriks et al., 2016) \\
\hline Spheroid & HepaRG & Monoculture & Repeated & 8 days & 16 & (Hendriks et al., 2016) \\
\hline Perfused liver-chip & $\mathrm{PHH}$ & Coculture with NPCs & Repeated & 14 days & 28 & (Novik et al., 2017) \\
\hline Spheroid & HepaRG & Monoculture & Repeated & 7 days & $\sim 40$ & (Liu et al., 2018a) \\
\hline Spheroid & HepG2 & Monoculture & Single & $24 \mathrm{~h}$ & 43 & (Mueller et al., 2014) \\
\hline Spheroid & HepaRG & Monoculture & Single & $24 \mathrm{~h}$ & 98 & (Mueller et al., 2014) \\
\hline MPCC & $\mathrm{PHH}$ & Monoculture & Single & $24 \mathrm{~h}$ & 100 & (Khetani and Bhatia, 2008) \\
\hline Spheroid & HepaRG & Monoculture & Single & $24 \mathrm{~h}$ & 120 & (Wang et al., 2015) \\
\hline
\end{tabular}


TABLE 1-Continued

\begin{tabular}{|c|c|c|c|c|c|c|}
\hline \multicolumn{7}{|c|}{ TABLE 1-Continued } \\
\hline Cell Model & Cell Type & Mono-/Coculture & Exposure Regimen & Exposure Time & $\mathrm{TC}_{50}$ & Reference \\
\hline \multicolumn{7}{|c|}{$\begin{array}{l}\text { Toxicity Mechanism: Mitochondrial Depletion } \\
\text { Fialuridine }\left(\mathrm{C}_{\max }=0.64 \mu \mathrm{M} ; \mathrm{TC}_{50} \text { in } \mu \mathrm{M}\right)\end{array}$} \\
\hline Spheroid & $\mathrm{PHH}$ & Monoculture & Repeated & 28 days & 0.1 & (Bell et al., 2016) \\
\hline Spheroid & $\mathrm{PHH}$ & Monoculture & Repeated & 32 days & 0.28 & (Hendriks et al., 2019) \\
\hline Spheroid & $\mathrm{PHH}$ & Monoculture & Repeated & 14 days & 0.7 & (Bell et al., 2016) \\
\hline Spheroid & $\mathrm{PHH}$ & Monoculture & Repeated & 14 days & $\sim 3$ & (Bell et al., 2018) \\
\hline Spheroid & $\mathrm{PHH}$ & Monoculture & Repeated & 7 days & 7.86 & (Hendriks et al., 2019) \\
\hline Spheroid & $\mathrm{PHH}$ & Coculture with NPCs & Repeated & 10 days & 77 & (Foster et al., 2019) \\
\hline Perfused liver-chip & $\mathrm{PHH}$ & Coculture with LSECs & Repeated & 10 days & 84 & (Foster et al., 2019) \\
\hline Spheroid & PHH & Coculture with NPCs & Repeated & 14 days & $>100$ & (Richert et al., 2016) \\
\hline Spheroid & HepG2 & Monoculture & Repeated & 4 days & 200 & (Gaskell et al., 2016) \\
\hline
\end{tabular}

hESC, human embryonic stem cell.

scale comparative data of microphysiological liver systems compared with spheroids and sandwich culture indicate that the sensitivity of microfluidic devices is similar (Foster et al., 2019) or lower (Rubiano et al., 2021). Thus, in the absence of larger screens, it remains thus unclear whether microfluidic systems have increased or decreased predictive accuracy compared with other organotypic liver models.

Overall, across 3D liver models, most hepatotoxicity data are available for spheroids. Acetaminophen, amiodarone, chlorpromazine, and fialuridine are the most tested compounds and might thus provide suitable reference values for the comparison of platform sensitivities (Table 1). Notably, commercial providers seem to focus predominantly on microfluidic chips, likely at least in part because of the substantial revenue associated with the sales of associated devices and equipment. Although these systems enable the study of gradients and liver zonation, the available data do not seem to indicate that these technically more demanding setups currently translate into improved phenotypes or increased predictive power, and further unbiased studies are required to comprehensively benchmark the available models.
2. Pharmacokinetic Studies. For approximately $70 \%$ of small-molecule drugs, metabolism is the main route of drug elimination, which mainly occurs in the liver (Wienkers and Heath, 2005). Therefore, hepatic intrinsic clearance $\left(\mathrm{CL}_{\text {int }}\right)$ prediction, identification of metabolites, and delineation of biotransformation routes constitute key elements of drug discovery and development pipelines. Currently, mainly microsomes and primary hepatocytes in suspension culture are used for pharmacokinetic profiling. However, stable metabolic activity in both microsomal preparations and hepatocytes in suspension is limited to several hours, making measurements of $\mathrm{CL}_{\text {int }}$ for slowly metabolized compounds difficult. For the analysis of low-clearance compounds, MPCCs and, to a lesser extent, microfluidic chips and spheroid systems have been presented (Table 2).

Using PHHs in MPCCs, $\mathrm{CL}_{\text {int }}$ could be predicted for $72 \%-76 \%$ within 3-fold of in vivo, which further increased to $92 \%$ if correction for protein binding was only integrated for low-clearance compounds (Qian et al., 2016; Chan et al., 2013). When stratifying by the extent of metabolism, clearance prediction was within 2 -fold for $70 \%$ of 10 tested low-clearance drugs, whereas $57 \%$ of 7 tested intermediate-clearance drugs

TABLE 2

Overview of studies using organotypic liver cultures for hepatic clearance predictions

\begin{tabular}{|c|c|c|c|c|c|}
\hline \multirow[b]{2}{*}{ Culture Paradigm } & \multicolumn{2}{|c|}{ Number of Compounds Predicted within 2-Fold of In Vivo $\mathrm{Cl}$} & \multirow[b]{2}{*}{ Prediction Method } & \multirow[b]{2}{*}{ Results/Conclusion } & \multirow[b]{2}{*}{ Reference } \\
\hline & $\begin{array}{l}\text { Low-Clearance } \\
\text { Compounds }\end{array}$ & $\begin{array}{l}\text { Medium- to High- } \\
\text { Clearance Compounds }\end{array}$ & & & \\
\hline $\mathrm{PHH}$ in MPCC & $7 / 10$ & $4 / 7$ & Conventional & $\begin{array}{l}\text { Medium- to high-Cl } \\
\text { compounds mainly } \\
\text { underpredicted }\end{array}$ & (Chan et al., 2013) \\
\hline $\mathrm{PHH}$ in MPCC & $9 / 15$ & $3 / 10$ & Conventional & $\begin{array}{l}\text { High-Cl compounds } \\
\text { underpredicted }\end{array}$ & $\begin{array}{c}\text { (Da-silva et al., } \\
\text { 2018) }\end{array}$ \\
\hline PHH in MPCC & $5 / 8$ & - & Direct scaling & No clear tendency & $\begin{array}{c}\text { (Kratochwil et al., } \\
\text { 2017) }\end{array}$ \\
\hline PHH in MPCC & $4 / 12$ & $2 / 3$ & Conventional & $\begin{array}{c}\text { Clinical drug } \\
\text { candidates (low Cl) } \\
\text { were better predicted } \\
\text { using direct scaling }\end{array}$ & $\begin{array}{c}\text { (Kratochwil et al., } \\
\text { 2017) }\end{array}$ \\
\hline PHH spheroids & $2 / 3$ & $1 / 3$ & Conventional & $\begin{array}{l}\text { Compounds were } \\
\text { underpredicted }\end{array}$ & $\begin{array}{c}\text { (Kanebratt et al., } \\
\text { 2021) }\end{array}$ \\
\hline PHH in chip & $0 / 2$ & $3 / 4$ & Direct scaling & $\begin{array}{l}\text { Low-Cl compounds } \\
\text { below LOQ, high } \mathrm{Cl} \\
\text { underpredicted }\end{array}$ & (Chao et al., 2009) \\
\hline
\end{tabular}

$\mathrm{Cl}$, clearance; LOQ, limit of quantification. 
were within the same interval (Chan et al., 2013). Comparisons of MPCCs with suspension and 2D cultures indicated that MPCCs considerably reduced confidence intervals and exhibited superior clearance predictions except for uptake transporter substrates for which 2D cultures showed the best performance (Lin et al., 2016; Da-silva et al., 2018; Docci et al., 2019, 2020; Umehara et al., 2020). Furthermore, using MPCC PHH data as input for pharmacokinetic modeling using the well stirred model, $75 \%$ of compounds were predicted within 3-fold, whereas clearance measurements were considerably lower in HepG2 and iPSC-derived HLCs (Kratochwil et al., 2017). The system moreover allows profiling the responsible drug-metabolizing enzymes of slowly metabolized compounds using P450 inhibition assays based on isoform-specific small molecules (Smith et al., 2021).

Besides MPCCs, intrinsic clearance measurements have been presented using microfluidic chips. Since flow has been integrated in the device, a mathematical modeling using the well stirred model was not necessary, but measured clearance data could be scaled up directly. Clearance predictions of slowly cleared drugs using the $\mathrm{H} \mu \mathrm{REL}$ coculture system of liver (HepaRG cells or human hepatocytes) and stromal cells were superior compared with suspension cultures, whereas both models performed similarly for intermediate clearance compounds (Chao et al., 2009; Bonn et al., 2016; Hultman et al., 2016).

For pharmacokinetic studies, liver spheroids have been primarily used to identify nuclear hormone receptor activation and P450 induction (Hendriks et al., 2020) to predict drug-drug interactions and to delineate species-specific induction mechanisms (Nudischer et al., 2020). In contrast, their use for clearance predictions is only emerging in recent years. In an industryled study using a small panel of four low-to-intermediate clearance compounds, intrinsic clearance could be predicted within 3-fold using as little as 6000 hepatocytes (Kanebratt et al., 2021). In agreement with these findings, clearance of a nonoverlapping set of seven lowand intermediate-clearance compounds was accurately predicted with an average fold error of 0.7 (Riede et al., 2021). Irrespective of the model, the turnover of highclearance compounds tended to be underpredicted, whereas low-clearance compound predictions were more accurate. Prediction accuracy was also dependent on the protein binding assumption and therefore the prediction method (Table 2). However, clearance studies are highly heterogeneous with regard to medium composition, hepatocyte concentration, and culture time, resulting in large interlaboratory and interexperimental variability. Thus, protocol standardization and in-depth benchmarking, ideally in a multicenter setting, are required to further develop 3D liver models for clearance predictions (Louisse et al., 2020).
In addition to clearance estimations, identification of the route of metabolism and the resulting metabolites is of importance to establish a full safety profile. Conventional methods often fail to predict the full metabolite profile, mainly because they are shortlived and lack complexity (Anderson et al., 2009). For instance, only $22(39 \%), 26(46 \%)$, and $31(55 \%)$ of 56 major metabolites corresponding to 27 different drugs could be detected in incubations of liver microsomes, liver S-9 fraction, and human hepatocyte suspensions, respectively (Dalvie et al., 2009). In contrast, MPCCs were able to detect $82 \%$ and $75 \%$ of the same excretory and circulating metabolites, respectively, after 1 week of exposure (Wang et al., 2010). Furthermore, MPCCs using primary hepatocytes of preclinically relevant animal models could replicate species differences in rate and extent of metabolism and guide preclinical model selection (Ballard et al., 2016; Kamel et al., 2021).

Also, spheroids can be used for metabolite profiling. For instance, $\mathrm{PHH}$ spheroids generated the known phase I and phase II metabolites of acetaminophen, diclofenac, lamotrigine, midazolam, propranolol, and salbutamol (Ohkura et al., 2014). Especially the identification of salbutamol-4- $O$-sulfate and lamotrigine$\mathrm{N}$-glucuronide were remarkable, as those metabolites had been identified in man in vivo but were not generated in S-9 fractions, microsomal preparations, or hepatocyte suspension cultures. In addition, 14 out of 16 major human phase I and phase II metabolites of carvedilol, imipramine, diazepam, disopyramide, sildenafil, and $S$-warfarin were detected using spheroid cultures (Kanebratt et al., 2021). Although these data show that organotypic liver models can predict the metabolites for a given compound of interest, they remain qualitative and do not allow to accurately estimate the quantitative metabolite profile in vivo.

In recent years, human liver chimeric mice in which the murine liver is ablated and repopulated by transplanted human PHHs have emerged as additional models for pharmacokinetic analyses (Peltz, 2013; Bissig et al., 2018). These models allow the study of human drug metabolism in an in vivo context. However, their low throughput and high costs in addition to the lack of clear demonstration of added value compared with organotypic and microphysiological liver systems have so far prevented more widespread usage of these models.

3. Liver Disease Modeling. Accurate modeling of hepatic diseases facilitates the identification of novel therapeutic targets and the screening for drug candidates. In recent years, organotypic liver models have been developed for a multitude of liver diseases, including nonalcoholic fatty liver disease (NAFLD), fibrosis, and various infectious diseases, such as 
malaria, hepatitis $\mathrm{B}$ virus (HBV), hepatitis $\mathrm{C}$ virus, and SARS-CoV-2 infections.

NAFLD constitutes a spectrum of symptoms and diseases from benign steatosis to NASH, fibrosis, and eventual cirrhosis. Etiology and progression of NAFLD are closely linked to obesity and type 2 diabetes, and it has become the leading cause of chronic liver disease worldwide with a global prevalence rate of $25 \%$ (Younossi et al., 2016). Although NAFLD, NASH, and fibrosis have been major targets of drug development efforts, all drug candidates have so far failed to receive regulatory approval, likely at least in part because of a lack of translatability of traditionally used genetic and diet-induced rodent models (Drenth and Schattenberg, 2020). Thus, the development of model systems that can mimic the pathobiology of human NAFLD constitutes a main frontier of organotypic liver model research (Müller and Sturla, 2019; Hundertmark and Tacke, 2020; Soret et al., 2020).

NAFLD and NASH have been predominantly studied in spheroids and microfluidic chips; however, limited MPCC data have been presented. Although the majority of systems use cocultures of $\mathrm{PHH}$ and primary NPCs, other approaches using rat hepatocytes (Bulutoglu et al., 2019) or hepatic cell lines (Gori et al., 2016; Suurmond et al., 2019) have been presented.

In vitro, NAFLD can be emulated by supplementing culture media with pathophysiologically relevant levels of carbohydrates and free fatty acids as well as high concentrations of insulin. In PHH spheroids, NAFLD models recapitulate steatosis, insulin resistance, reduced glucose consumption, and the induction of genes involved in de novo lipogenesis and gluconeogenesis (Kozyra et al., 2018; Kemas et al., 2021) (Fig. 3A). Importantly, these parameters are reversible and allow for the evaluation of antisteatotic effects of drug candidates or dietary supplements (Kozyra et al., 2018; Cordero-Herrera et al., 2019). Furthermore, spheroids recapitulate the effects of genetic risk factors, such as variations in TM6SF2 (Prill et al., 2019) or PNPLA3 (Pingitore et al., 2019; Hurrell et al., 2020), allowing for targeted drug development for specific at-risk populations.

Coculture spheroids moreover recapitulate interactions between PHH and NPCs and allow the study of disease progression from steatosis to NASH and fibrosis, as evident by stellate cell activation ( $\alpha$ SMA) and collagen 1A1 deposition (Hurrell et al., 2020). These features allow the screening for compounds that attenuate endpoints of interest, such as steatosis, inflammation, or fibrogenesis, and facilitate the identification of drug targets with potential therapeutic relevance in a human context (Fig. 3B). For instance, in obesityinduced insulin resistance liver macrophages increase secretion of multiple noninflammatory factors, and experiments in spheroids showed that one of these, IGFBP7, regulates hepatocyte lipogenesis and gluconeogenesis, thus providing an interesting and conceptually novel target for the treatment of hepatic insulin resistance and NAFLD (Morgantini et al., 2019). Furthermore, coculture liver spheroids recapitulated the increased expression of miR-144 seen in obesity, which in turn represses IRG1 (Azzimato et al., 2021). IRG1 catalyzes the production of itaconate, an inhibitor of succinate dehydrogenase and fumarate hydratase, and its downregulation causes depletion of fumarate, reduced activity of the antioxidant master regulator NRF2, and exacerbation of hepatic oxidative stress. Notably, downregulation of miR-144 in either PHH or NPCs could rescue obesity-induced suppression of NRF2, highlighting miR-144 as an interesting target to restore the endogenous antioxidant response in obesity-associated NAFLD (Azzimato et al., 2020). In addition to their utility for the identification of new therapeutic modalities, liver spheroids from specific patient populations also allow testing impacts of disease states or genetic polymorphisms on drug metabolism and toxicity (Ingelman-Sundberg and Lauschke, 2018).

NAFLD can furthermore be mimicked in microfluidic devices using similar cocultures of $\mathrm{PHHs}$, stellate cells, and macrophages in high-nutrient, high-insulin conditions. Specifically, these culture conditions resulted in hepatic lipid accumulation and lipidomic signatures similar to NASH biopsies as well as increased TGF $\beta$ levels and hepatic stellate cell (HSC) activation (Feaver et al., 2016). Furthermore, the model corroborated in vivo observations that BMP8B might be an important contributor to stellate cell activation and NASH progression (Vacca et al., 2020). In addition to steatosis, exposure to elevated levels of free fatty acids resulted in altered gene expression and metabolic activity (Fig. 3C). Treatment of steatotic cultures with metformin resulted in reduced free fatty acid uptake and lower cellular fat content (Kostrzewski et al., 2017). Hepatic fat accumulation resulted in the generation of an inflammatory milieu with elevated expression of profibrotic markers, which could be ameliorated by treatment with the FXR agonist obeticholic acid (Kostrzewski et al., 2019). Furthermore, as in spheroids, the PNPLA3 mutation I148M enhanced the overall NASH phenotype. Using a different threechannel fluidic device, exposure to lipotoxic stress induced steatosis, hepatocellular ballooning, HSC activation, and elevation of inflammatory and profibrotic markers, effects that could be blunted using the dual PPAR $\alpha / \delta$ agonist elafibranor (Freag et al., 2020).

Although fewer studies have been described about the modeling of NASH in MPCCs, the available data indicate that steatosis and insulin resistance can be induced by culture in hyperglycemic media, similar to spheroids and microphysiological liver models (Davidson et al., 2016). Moreover, coculturing conventional MPCCs with activated stellate cells resulted in increased levels of IL6 and hepatocyte dysfunction, which could be alleviated by treatment with 

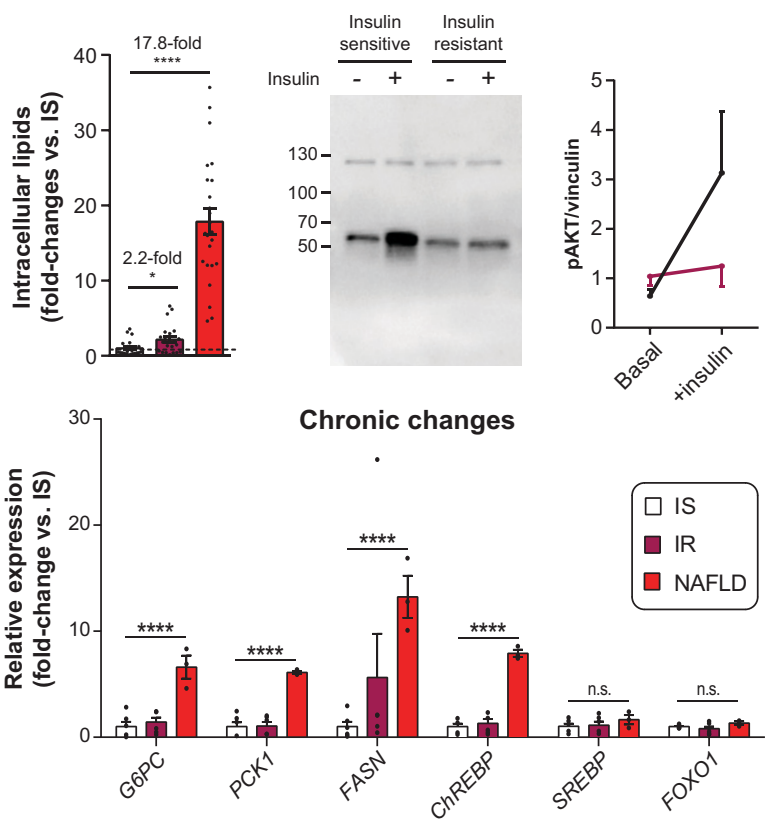

Fibrosis

B
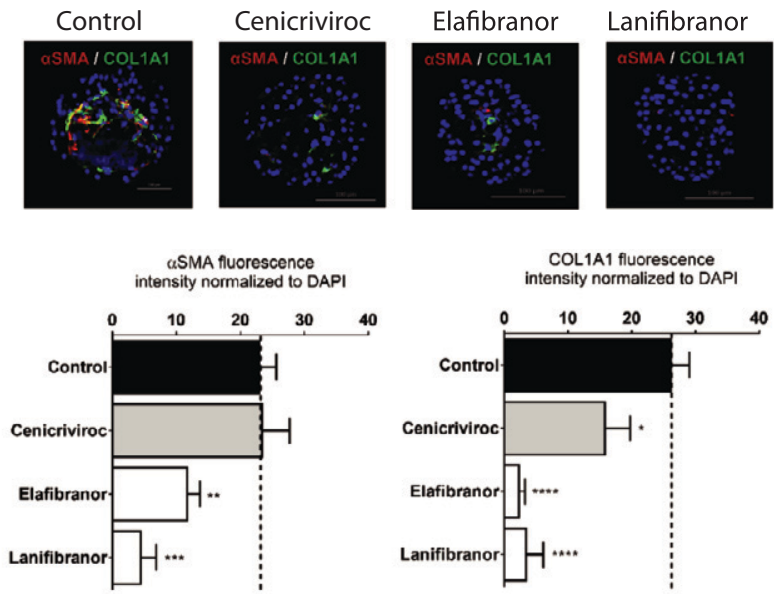
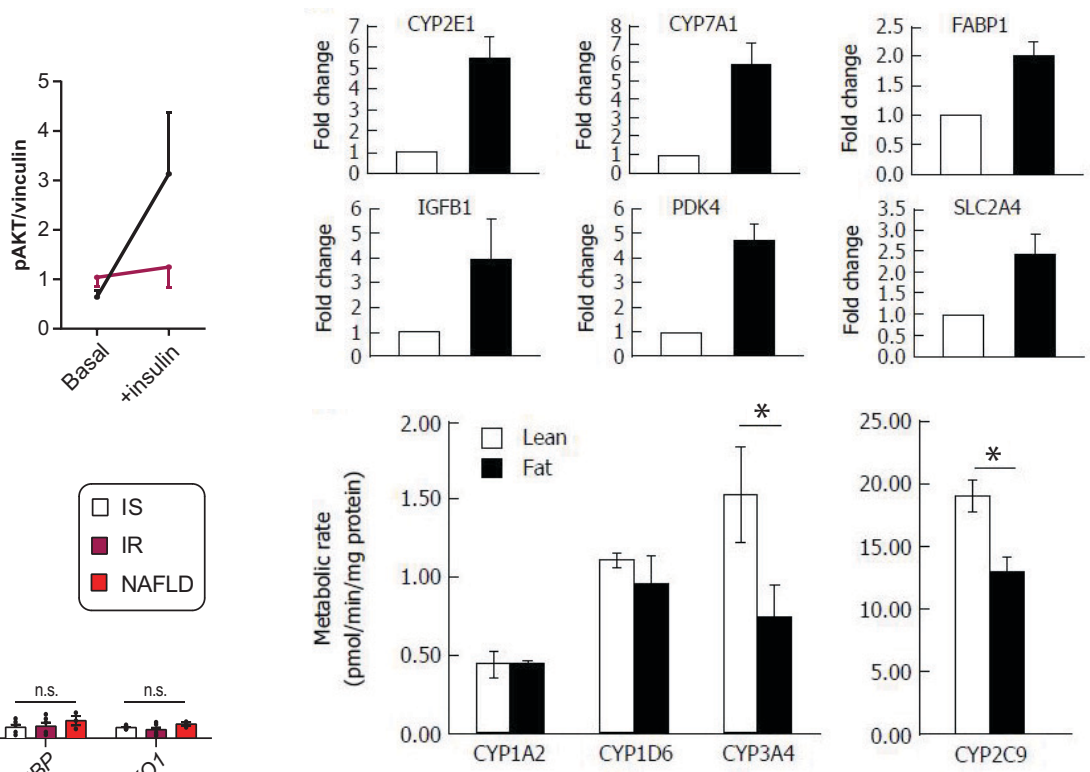

Hepatitis B virus infection

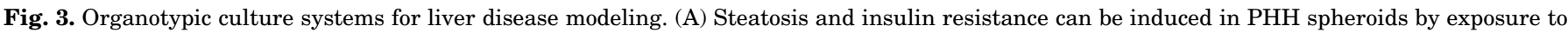

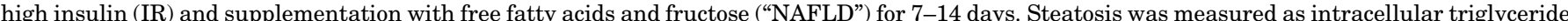

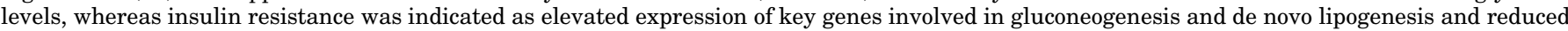

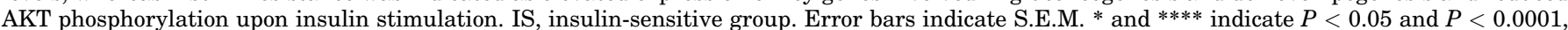

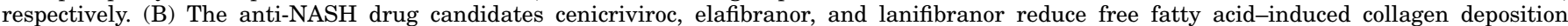

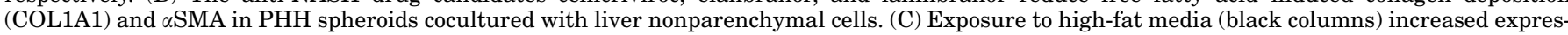

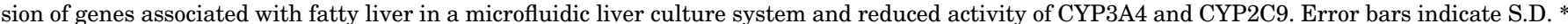

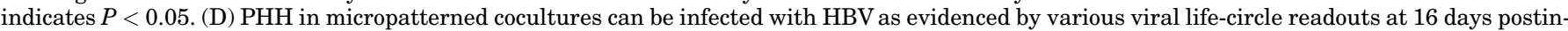

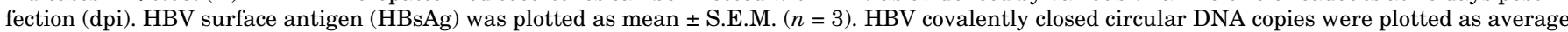

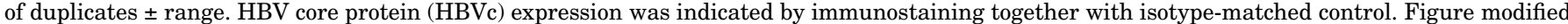
with permission from (Hurrell et al., 2020; Kemas et al., 2021; Kostrzewski et al., 2017; Shlomai et al., 2014).

obeticholic acid or the NADPH oxidase inhibitor setanaxib (Davidson et al., 2017).

In addition to NAFLD, NASH, and fibrosis, liver models have been used in drug development for hepatotropic protozoan infections. The life cycle of Plasmodium falciparum, berghei, or vivax malaria parasites from infection to the release of hepatic merozoites can be emulated in both MPCCs (March et al., 2013; Gural et al., 2018) and spheroids (Arez et al., 2019; Chua et al., 2019). Although various cell models are compatible with malaria infections, high and stable expression of CD81 and SRB1 is required for efficient sporozoite progression, which favors $\mathrm{PHH}$ over hepatoma cell lines and iPSC-derived HLCs (March et al., 
2013; Ng et al., 2015). Importantly, spheroids provide a scalable translational tool for the development of antiplasmodial therapies as demonstrated by close agreement of in vitro and in vivo efficacy data of the Plasmodium Elongation Factor 2 Inhibitor M5717 (Arez et al., 2019). Similarly, MPCCs allowed for the testing of antihypnozoite drugs for prophylactic or cidal therapies (Gural et al., 2018). In addition to malaria, a sandwich culture of liver sinusoidal endothelial cells and Huh-7 cells in a 3D collagen matrix sandwich recapitulates infection with the protozoan parasite Entamoeba histolytica (Petropolis et al., 2014).

Organotypic 3D models have been applied to a multitude of viral liver diseases. HBV infection relies on expression of the viral receptor NTCP (SLC10A1). HBV infection models have been established in $\mathrm{PHH}$ MPCCs that recapitulate productive viral infection, including viral gene expression, and enable the detection of HBV core protein throughout the 3-week culture period (Fig. 3D) (Shlomai et al., 2014). In addition, HBV infection can be successfully modeled in microfluidic chips (OrtegaPrieto et al., 2018) and iPSC-derived HLC spheroids (Fu et al., 2019), and the authors show that the model can replicate antiviral effects of entecavir, a reverse-transcriptase inhibitor that interferes with viral replication, and the NTCP substrate tauroursodeoxycholic acid that blocks HBV entry. Similarly, Huh-7 and PHH spheroids (Murakami et al., 2006; Ananthanarayanan et al., 2014) as well as PHH MPCCs (Ploss et al., 2010) are permissive to hepatitis $\mathrm{C}$ virus infection and allow for miR-122-dependent viral replication. Recently, liver spheroids were furthermore used to evaluate hepatotoxicity of antiviral candidate drugs (Monteil et al., 2021) and to study extrapulmonary effects of SARS-CoV-2 infections (Stebbing et al., 2020). In this model, increased exposure to class I interferons, as typically observed during excessive inflammation in COVID-19, induced expression of the viral receptor ACE2, which in turn resulted in increased infectivity and altered expression of genes involved in coagulation and platelet activation (Stebbing et al., 2021). Importantly, these effects were blunted by therapeutic concentrations of the JAK/ STAT inhibitor baricitinib that had been suggested by artificial intelligence-based knowledge graphs to inhibit both cytokine signaling and viral endocytosis (Richardson et al., 2020) and contributed to the decision by FDA and Japanese Pharmaceuticals and Medical Devices Agency to grant emergency use authorization of baricitinib in combination with remdesivir for hospitalized adults with severe COVID-19.

\section{Kidney}

Kidneys play a crucial role in maintaining body homeostasis through filtration processes, secretion of waste, and reabsorption of water and essential small molecules. The structural unit of the kidney is the nephron, which comprises various cell types originating from different embryological lineages. The nephron is divided into glomerulus, proximal tubule, loop of Henle, the distal tubule, and collecting duct where filtered fluid is converted into urine. Filtration takes place in the glomerulus, whereas secretions and reabsorption occur in the rest of the nephron mainly in the proximal tubule.

Conventional 2D in vitro culture models from immortalized cell lines, such as human HEK293 and HK-2, porcine LLC-PK1 or canine MDCK, that have long been used to study drug transport and nephrotoxicity do not accurately mimic the cellular phenotypes and tissue microenvironment, which manifested in the poor translation of basic research

TABLE 3

Table with strengths/weaknesses of different models for areas of the nephron

\begin{tabular}{|c|c|c|c|c|}
\hline Nephron Part & Cell Origin & Applications & Pros & Cons \\
\hline \multirow[t]{3}{*}{ Proximal tubule } & Primary PTECs & $\begin{array}{l}\text { Drug transport and } \\
\text { nephrotoxicity }\end{array}$ & $\begin{array}{l}\text { TEER similar to native } \\
\text { tissue, metabolic enzymes, } \\
\text { and drug transporters } \\
\text { preserved }\end{array}$ & $\begin{array}{c}\text { Short-term cultures (up to } \\
2 \text { weeks max), limited } \\
\text { access to primary } \\
\text { material }\end{array}$ \\
\hline & iPSCs & $\begin{array}{l}\text { Drug transport and } \\
\text { nephrotoxicity }\end{array}$ & $\begin{array}{c}\text { Can be expanded, } \\
\text { compatible with long-term } \\
\text { culture }\end{array}$ & $\begin{array}{c}\text { Immature expression of } \\
\text { uptake transporters }\end{array}$ \\
\hline & Immortalized PTECs & $\begin{array}{l}\text { Drug transport and } \\
\text { nephrotoxicity }\end{array}$ & $\begin{array}{c}\text { Can be expanded ( } 30 \\
\text { passages), compatible with } \\
\text { long-term culture }\end{array}$ & $\begin{array}{l}\text { Immature expression of } \\
\text { uptake transporters }\end{array}$ \\
\hline \multirow[t]{2}{*}{ Glomerulus } & Primary podoctyes & Studies of podocytopathies & $\begin{array}{c}\text { Express slit diaphragm } \\
\text { proteins, patient-derived } \\
\text { podocyte cultures are } \\
\text { possible }\end{array}$ & $\begin{array}{l}\text { Limited access to primary } \\
\text { material }\end{array}$ \\
\hline & iPSCs & Study of podocytopathies & $\begin{array}{l}\text { Long-term cultures, } \\
\text { generation of isogenic lines }\end{array}$ & $\begin{array}{c}\text { Loss of foot processes and } \\
\text { slit diaphragms in most of } \\
\text { models }\end{array}$ \\
\hline $\begin{array}{l}\text { Distal tubule and } \\
\text { collecting duct }\end{array}$ & iPSCs & $\begin{array}{l}\text { Drug testing, disease } \\
\text { model }\end{array}$ & $\begin{array}{l}\text { Expression of } \mathrm{AQP} 2, \\
\text { response to toxins }\end{array}$ & $\begin{array}{l}\text { Most models show } \\
\text { incomplete maturation of } \\
\text { distal tubule and } \\
\text { collecting duct }\end{array}$ \\
\hline
\end{tabular}


into clinical therapies (Vriend et al., 2021). To overcome these limitations, models representing major segments of the nephron or specific domains have been generated from stem cells (Table 3). Models from stem cells allow studies to recapitulate the complexity of the human nephron, but cells remain phenotypically immature. In contrast, models based on primary cells can accurately reflect the function of their in vivo counterparts, but no primary system has yet been presented that contains all the nephrotic segments.

\section{A. Models of the Major Nephrotic Segments}

Various stem cell-based models have been developed in which iPSCs differentiate into various renal cell types and develop structures resembling the early kidney architecture (Little et al., 2019). iPSCs can be differentiated into complex kidney organoids that each contain glomeruli as well as the different regions of the tubule, using a complex differentiation regimen based on growth factors and small molecules (Morizane et al., 2015; Takasato et al., 2015; Taguchi and Nishinakamura, 2017). Although the molecular phenotype of organoids initially resembled fetal kidney, after extended maturation, adult structures formed, including WT1+ glomerular structures that connected to typical proximal tubular epithelium with apical brush borders, an UMOD+ loop of Henle, and an open lumen characteristic of distal tubule and collecting duct. Importantly, organoid cultures can be fully automated by running the entire 21-day protocol from plating to differentiation and analysis using liquid-handling robots in 384-well plates (Czerniecki et al., 2018). Using this platform, the authors treated gene-edited iPSC-derived organoids with mutations in polycystin-1 as genetic cause of polycystic kidney disease with eight compounds and show that blebbistatin-mediated inhibition of myosin contributes to cystogenesis, thus providing a technological framework for large-scale compound testing.

However, cisplatin toxicity in organoids was only observed at concentrations $\geq 5 \mu \mathrm{M}$, which is more than 20 fold higher than its therapeutic $\mathrm{C}_{\max }$ values $(\sim 200 \mathrm{nM})$. This reduced sensitivity is likely at least in part due to 10to 100 -fold reduced expression of important renal transporters, such as organic anion transporter (OAT) 1/2, MATE2$\mathrm{K}$, and OCT2 (Bajaj et al., 2018). Particularly the latter is important in nephrotoxicity as its inhibition was shown to have protective effects in vivo (Katsuda et al., 2010).

Alternatively, iPSCs could be differentiated into podocytes, proximal tubules, and distal tubules in either $2 \mathrm{D}$ or $3 \mathrm{D}$ culture using a protocol of subsequent transfection with two sets of synthetic mRNAs (Hiratsuka et al., 2019). These structures exhibited $\gamma$-glutamyl transferase activity and albumin uptake and were sensitive to gentamicin-induced nephrotoxicity, suggesting some extent of renal functionality.
Most of the above reported protocols resulted in proximal tubule cells with phenotypic and functional maturity similar to cells in the first trimester of kidney development. Maturation can be further facilitated by differentiation of iPSCs in 3D culture and exposure to soft hydrogel scaffolds with a Young modulus of $1 \mathrm{kPa}$ similar to native kidney (Garreta et al., 2019).

Importantly, however, whereas these iPSC-based protocols are successful in forming various nephritic cell types, approximately $10 \%-20 \%$ of formed cells are nonrenal, and off-target differentiation remains to be problematic even after protocol refinements (Wu et al., 2018; Combes et al., 2019). To overcome these problems, the use of adult stem cells from kidney tissue or human urine provides a promising method to develop pure tubular organoids in which all cells were kidney epithelial in nature (Schutgens et al., 2019). However, although these organoids are positive for proximal tubules, loop of Henle, distal tubules, and collecting duct, they lack glomerular structures. Alternatively, more targeted differentiation protocols have been developed that focus on the generation of specific renal cell types, thus trading off tissue model complexity against the purity of the resulting cell population.

\section{B. Proximal Tubule Models}

The proximal tubule accounts for $70 \%$ of renal reabsorption and constitutes the main site of drug-induced adverse effects in the kidney (Nigam et al., 2015). Several renal proximal tubule cell models have been developed from primary human proximal tubule epithelial cells (PTECs), human renal progenitor cells, or pluripotent stem cells, which, in turn, can be combined with multiple different culture systems, such as 2D transwell membrane culture, hollow fiber bioreactors, or microfluidic chips.

Various protocols have been generated that allow the generation of proximal tubule cells from either ESCs or iPSCs. ESC-derived proximal tubule cells achieved approximately 50\% of activity of human PTECs as assayed by response to parathyroid hormone, $\gamma$-glutamyl transferase activity, and ammonia production; however, the fraction of cells positive for the proximal tubule epithelium marker aquaporin (AQP) 1 was only $38 \%$ (Narayanan et al., 2013). By contrast, from iPSCs, proximal tubule cells can be differentiated with $>90 \%$ purity based on the markers AQP1, sodium-glucose transporter protein (SGLT) 1, GLUT1, OAT3, PEPT1, Na+/K+ ATPase, URO10, and ZO-1 (Kandasamy et al., 2015).

To model filtration and reabsorption of the human proximal tubule, a 3D model was developed in which immortalized human PTECs were seeded with glomerular microvascular endothelial cells in a permeable ECM scaffold (Lin et al., 2019) (Fig. 4A). Although the system was not comprehensively characterized with 
A
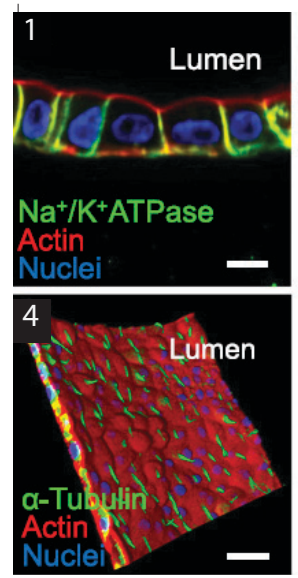

C

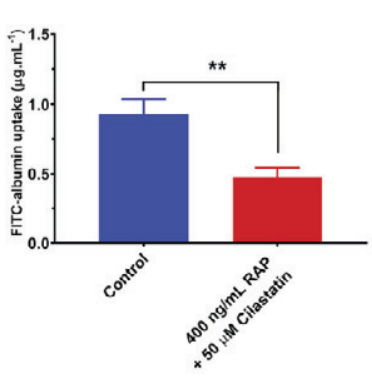

5
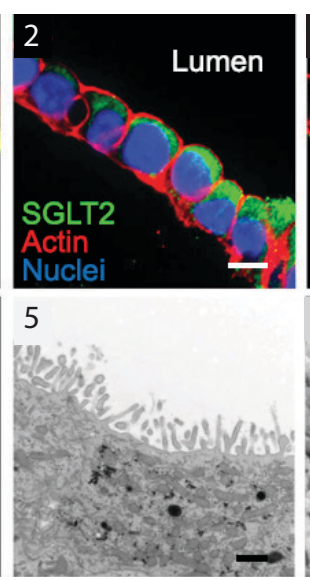

D

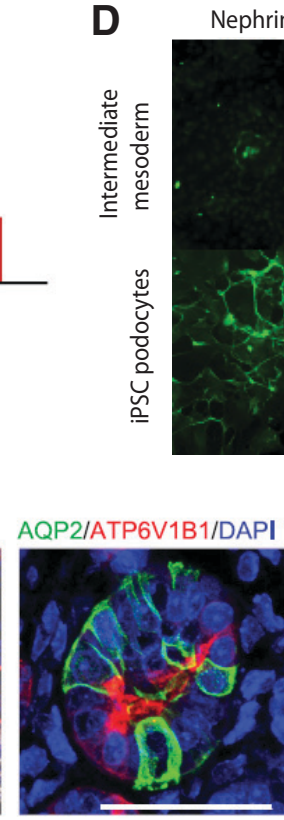

F

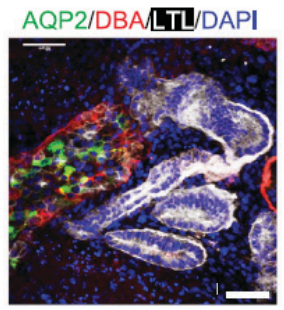

MEIS1/PDGFR $\beta / L T L / D A P I$

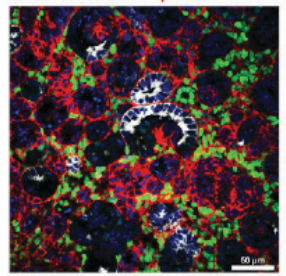

SLC12A1/ECAD/DAPI

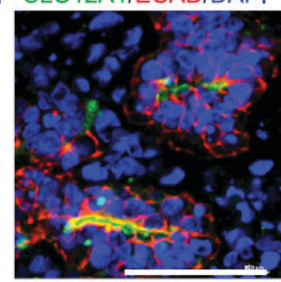

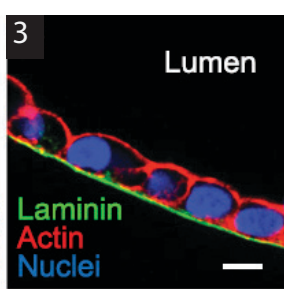
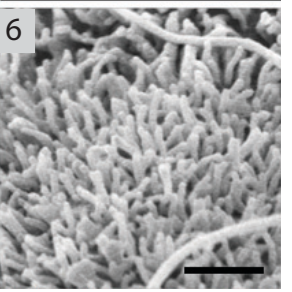

WT1

PAX2

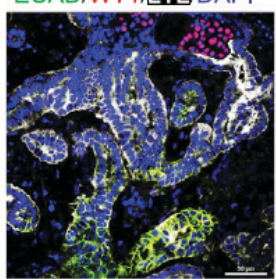

CD31/NPHS1/LTL/DAPI

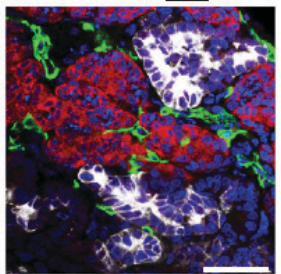

B

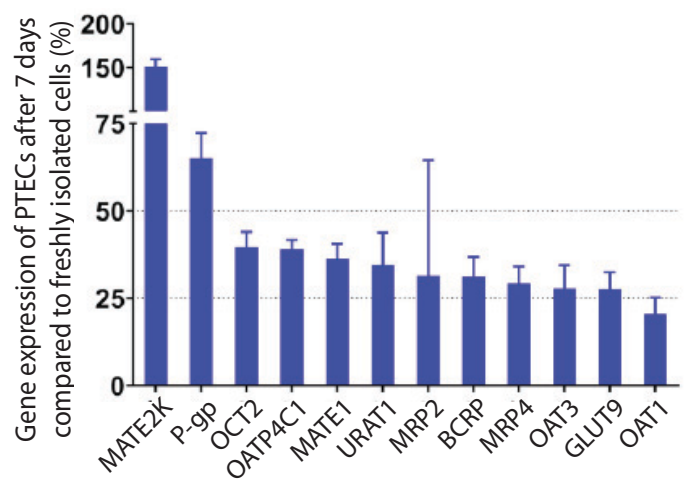

E

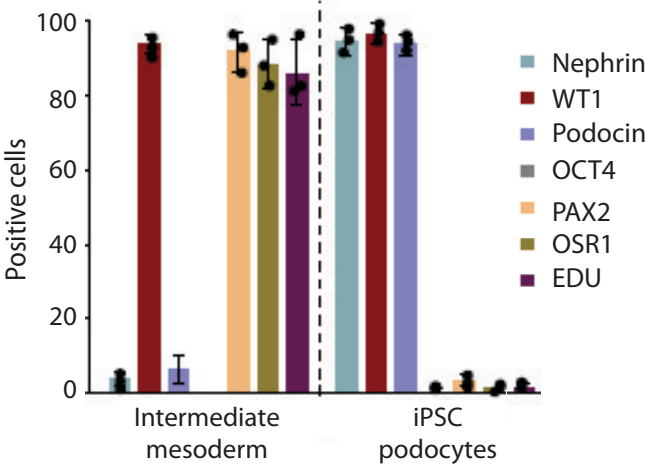

G

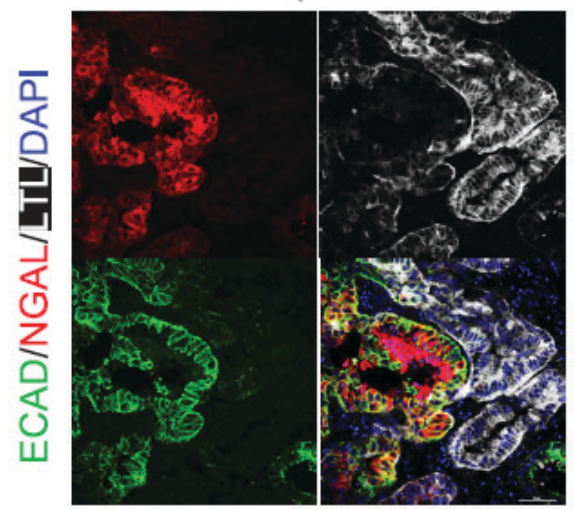

Fig. 4. Overview of models of the human nephron. (A) Fluorescence images of immortalized human PTECs cocultured with glomerular microvascular endothelial cells showing basolateral expression of $\mathrm{Na}+/ \mathrm{K}+$ ATPase (1) and apical expression of the glucose transporter SGLT2 (2) and deposition of a laminin-rich basement membrane (3). Furthermore, cells featured $\alpha$-tubulin positive primary cilia (4). Transmission electron microscopy (5) and scanning electron microscopy (6) images showing dense carpets of microvilli. Scale bars for $1-4=10 \mu \mathrm{m}$; scale bars for $5-6=1 \mu \mathrm{m}$. (B) Expression of major renal transporters remains stable in primary human PTEC transwell cultures for at least 7 days. (C) Uptake of FITC-labeled albumin is sensitive to receptor-associated protein (RAP) and cilastatin, inhibitors of megalin/cubulin endocytosis, showing functionality of these endocytic receptors. Error bars indicate S.E.M. ** indicates $P<0.01$. (D) Human iPSC-derived podocytes express nephrin and WT1, key markers of a mature phenotype, and lose expression of the intermediate mesoderm marker PAX2. (E) Quantification of changes in protein expression. Differentiated cells are positive for the podocyte markers nephrin, WT1, and podocin, whereas they are negative for the pluripotency marker OCT4 and the progenitor cell markers PAX2 and OSR1. Furthermore, iPSC-derived podocytes are nonproliferative as indicated by a lack of EdU incorporation. (F) Organoids differentiated using vasopressin and aldosterone show expression of the collecting duct markers (AQP2, DBA, and ATP6V1B1) as well as of proximal tubules (LTL), distal tubules (ECAD and SLC12A1), podocytes (WT1 and NPHS1), endothelial cells (CD31), and stroma (MEIS1 and PDGFR $\beta)$. Scale bars = 50 $\mu \mathrm{m}$. (G) Response of collecting duct organoids to cisplatin showed upregulation of the proximal tubule injury marker HAVCR1 in LTL-positive proximal tubule cells and distal tubule injury marker NGAL in E-cadherin-positive distal tubules. Figure modified with permission from (Bajaj et al., 2020; Lin et al., 2019; Uchimura et al., 2020). 
regards to molecular phenotypes, it allowed for longitudinal quantification of albumin uptake and could mimic the reduced glucose reabsorption upon exposure to the SGLT2 inhibitor dapagliflozin.

Because of their mature molecular phenotypes and intact transporter function, freshly isolated PTECs remain the gold-standard cell model for emulating transepithelial flux and nephrotoxicity. Transwell cultures of PTECs expressed the renal transporters SGLT1, SGLT2, OCT2, OCTN2, OAT1, OAT3, OAT4, MDR1, MRP2, and BCRP for at least 10 days in culture, remained endocytotically active, and retained para-aminohippurate $(\mathrm{PAH})$, creatinin, and fluorescein secretory functions (Brown et al., 2008; Sanchez Romero et al., 2020) (Fig. 4, B and C). However, cells rapidly lose their columnar shape and polarization (Elwi et al., 2009). To overcome these limitations, microfluidic chips were developed in which PTECs are subjected to apical shear stress. To this end, PTECs can be cultured in hollow tubes (Ng et al., 2013; Weber et al., 2016) or on porous collagen IV-coated membranes that separate apical and basal flow channels (Jang et al., 2013). In both models, perfusion increased tight junction formation and transporter expression as well as PAH translocation compared with static cultures. Furthermore, $\mathrm{PAH}$ transport was sensitive to the OAT transporter inhibitor probenecid in perfused but not in static culture (Weber et al., 2016). However, all these devices were fabricated from PDMS, which raises concerns about drug absorption.

\section{Glomerulus Models}

The development of glomerular model is complicated by the limited access to podocytes as well as by the structural complexity of the glomerular filtration barrier. Most research has been conducted using immortalized podocyte cell lines. However, these do not develop secondary foot processes and do not form secondary processes and show only a very limited expression of glomerular markers (Hagmann and Brinkkoetter, 2018). Relatively pure (efficiency >90\%) podocyte-like cells can be generated from iPSCs in 2D culture using a chemically defined differentiation cocktail of growth factors and small molecules (Song et al., 2012a; Musah et al., 2017). After 21 days of differentiation, the cells expressed key glomerular markers, such as nephrin and WT1, and developed elongated podocin-positive protrusions (Fig. 4, D and E). Upon coculture of podocytes and endothelial cells on opposite sides of a porous stretchable membrane, which separated two flow channels corresponding to urinary ducts and renal endothelium, the device retained albumin in the vasculature channel, whereas inulin clearance rates were identical to in vivo estimates (Musah et al., 2017). Exposure to clinically relevant concentrations of doxorubicin resulted in dose-dependent delamination of podocytes, disrupted barrier function, and increased levels of albumin in the urinary channel, mimicking glomerular injury and proteinuria. Notably, fluidic shear stress due to flow enhanced podocyte maturation as evidenced by higher levels of nephrin and synaptopodin expression as well as increased formation of foot process-like structures compared with static culture (Homan et al., 2019). In contrast, the application of light pressures of $4-8 \mathrm{mmHg}$ reduced expression of WT1 and the differentiation marker KLF15 in primary human podocytes while increasing expression of fibronectin, an indicator of podocyte fibrosis (Yu et al., 2020).

In a microfluidic coculture system of endothelial cells and iPSC-derived podocytes or primary human podocytes obtained from discarded kidneys harvested from patients without obvious renal disease, the different cell types were separated in adjacent channels separated by a collagen I layer (Petrosyan et al., 2019). The authors show that the system retained selective permeability to albumin for at least 1 hour after introduction of FITC-conjugated albumin. Notably, primary cell cultures retained this selective permeability for at least 28 days in culture, whereas iPSC-derived podocytes deteriorated in less than 2 weeks. In agreement with these observations, subsequent analyses suggested that differentiation of podocytes in $2 \mathrm{D}$ culture remains limited, resulting in phenotypes similar to conditionally immortalized human podocytes (Hale et al., 2018). In contrast, differentiation in $3 \mathrm{D}$ culture was reported to increase expression of podocyte markers, such as WT1, PODXL, and NPHS1/2, by multiple orders of magnitude. 3D differentiated glomeruli were at least as sensitive to doxorubicin as differentiated $2 \mathrm{D}$ cultures $(<400 \mathrm{nM})$ and allowed to recapitulate podocytopathy using iPSCs from a patient with type I nephrotic syndrome that manifested as podocyte hypertrophy with reduced expression of nephrin and podocin. Combined, these data indicate that primary or $3 \mathrm{D}$ differentiated podocytes are required to achieve long-term stable and physiologically relevant models for studies of glomerular filtration, whereas 2D differentiated podocytelike cells might be sufficient to accurately mimic nephrotoxicity.

\section{Distal Tubule and Collecting Duct Models}

Only few studies have focused on the development of dedicated models of distal tubules or collecting ducts. In early work, MDCK cells isolated from canine kidney tubules were cultured in perfused PDMS chips (Baudoin et al., 2007). Cells remained viable and proliferative; however, as cells were cultured on the fibronectin-coated surface of the chip and not on membranes, permeability or barrier function could not be evaluated. Culturing primary rat collecting duct cells on a porous membrane support in a perfused microchannel resulted in enhanced cell polarization and improved functionality within smaller-diameter fibers as indicated by increased water 
and sodium ion uptake after stimulations with vasopressin and aldosterone, respectively (Jang and Suh, 2010). Although no human model systems have been developed specifically for distal tubules or collecting ducts, iPSCderived organoid protocols have been used to develop mature collecting ducts (Uchimura et al., 2020). The method relies on the independent differentiation of iPSCs into metanephric mesenchyme and ureteric buds, whose combination then resulted in the formation of proximal and distal tubules, glomeruli, endothelial cells, and collecting duct-like structures upon induction with vasopressin and aldosterone (Fig. $4 \mathrm{~F}$ ). The organoids showed increased sensitivity to cisplatin with increased expression of the injury marker neutrophil gelatinaseassociated lipocalin (NGAL) in the distal regions of the tubule (Fig. 4G).

\section{E. Applications of 3D Human Kidney Models}

1. Drug Transporter Studies. Regulators in Europe, the United States, and Asia mandate that interactions of new compounds with key renal transporters are tested during preclinical drug development, but current systems do not meet the needs of pharmaceutical companies. Glomerular filtration, tubular secretion, and reabsorption must be considered when investigating the renal clearance of drugs and their metabolites. Using preclinical animal models, prediction of renal excretion works well when glomerular filtration is the major way of clearance. For tubular secretion and reabsorption, however, in vivo models are often inaccurate because of species differences in transporters and drug-metabolizing enzymes (Zou et al., 2018; Bueters et al., 2020). For kidney models to be of use for ADME applications, they should have polarized activity of OAT $1 / 3$ and OCT 2 on the basolateral side and MDR1, BCRP, MRP2, and MATE on the apical side, show barrier function, and feature appropriate reuptake transport and kidney-relevant metabolism (Fowler et al., 2020). Furthermore, the system should recapitulate known drug-drug interactions that influence drug transport or metabolism.

Common assays to evaluate renal solute carrier (SLC)mediated transport are based on $\mathrm{PAH}$ or creatinin, whereas ATP-binding cassette transporters $(\mathrm{ABC})$ transport is measured using acetoxymethyl-calcein or 5-chloromethylfluorescein diacetate as substrates of P-gp and MRPs, respectively. These dyes become fluorescent and membrane-impermeable only after intracellular removal of the acetomethoxy or chloromethyl groups by esterases. Renal metabolism is mostly tested by vitamin $\mathrm{D}$ biotransformation. Evaluation of at least some of those endpoints constitutes an essential element of the functional characterization of organotypic or microphysiological renal models. Importantly, however, although molecular phenotypes and cellular morphology are increasingly benchmarked against in vivo tissue or primary freshly isolated cells, most characterizations of transporter function or metabolism remain qualitative. For instance, OCT2, P-gp, and MRP activity was demonstrated in immortalized PTECs using the fluorescent substrates 4(4-(dimethylamino)styryl)- $N$-methylpyridinium iodide, acetoxymethyl-calcein, and 5-chloromethylfluorescein diacetate, respectively (Jansen et al., 2015; Vormann et al., 2018; Vriend et al., 2018). However, although the authors demonstrate that uptake is specific and can be blocked by inhibitors of the respective transporter, whether the transport kinetics quantitatively correspond to in vivo function remained unclear. Similarly, PTECs in perfusion cultures were able to bioactivate vitamin $\mathrm{D}$ at rates around 0.01 $\mu \mathrm{l} / \mathrm{h}$, but again, it was not analyzed whether these kinetics resemble the activity of proximal tubules in vivo (Weber et al., 2016).

In an interesting recent study, renal reabsorption of five compounds was determined in a perfused PTEC chip, and the resulting data were used to predict overall in vivo renal clearance using a physiologically based parallel tube model (Sakolish et al., 2020). For creatinine the model correctly predicted no reabsorption, which aligns with in vivo data that creatinine excretion is driven by the glomerular filtration rate. Furthermore, in vivo and chip data aligned for perfluorooctanoic acid, cisplatin, and cadmium clearance, whereas gentamicin reabsorption was underpredicted (20\% in vivo, $0.5 \%$ in PTEC chip). Similar attempts for alignments with physiologic data were performed for a microfluidic model of iPSC-derived glomerular cells in which glomerular filtration rates of albumin and inulin were benchmarked against in vivo values and shown to be indistinguishable (Musah et al., 2017). Overall, these data indicate that the currently available systems give promising results, but further characterization using liquid chromatography coupled with tandem mass spectrometry-based measurements of several compounds and benchmarking to in vivo renal clearance is needed.

2. Nephrotoxicity. Kidneys take part in the elimination of $32 \%$ of the 200 most prescribed drugs (Morrissey et al., 2013). Given these essential roles in drug clearance, drug-induced kidney injury (DIKI) is relatively common with frequencies of $14 \%-26 \%$ in adults and even higher numbers in pediatric populations (Awdishu and Mehta, 2017). Mechanisms and patterns of injury differ between drugs with aminoglycosides causing acute tubular necrosis, whereas puromycin and adriamycin are associated with glomerular injury, nonsteroidal anti-inflammatory drugs cause injury to the loop of Henle, and sulfonamides and amphotericin $\mathrm{B}$ result in predominant injury of the distal tubule (Pannu and Nadim, 2008). Current models used for toxicity studies often fail to predict nephrotoxicity (Tiong et al., 2014). Especially interspecies differences in renal transporter expression 
limit the use of animal models to study adverse drug effects (Chu et al., 2013).

The aforementioned organotypic kidney models using human cells aspire to improve the accuracy of nephrotoxicity predictions. To be of relevance for the pharmaceutical industry, novel microphysiological kidney models should incorporate the tissue mechanical environment (e.g., fluid shear and pressure) and allow the evaluation of different endpoints, such as filtration rate, barrier function, transport activity, viability, or release of kidney tubular injury biomarkers, such as KIM-1 (Phillips et al., 2020). However, as mentioned above, a complete and phenotypically accurate model that contains all segments of the kidney does not exist yet.

Since the proximal tubule is the major site of nephrotoxicity, most studies have used proximal tubule models. Using rat kidney scaffolds recellularized with conditionally immortalized human PTECs overexpressing OAT1, concentration-dependent nephrotoxicity of cyclosporine A, tenofovir, and the OCT2 substrate cisplatin was observed (Fedecostante et al., 2018). Furthermore, cells were more sensitive to tenofovir and cisplatin than in 2D culture, which aligned with increased expression of the respective transporters OAT1 and OCT2, respectively. However, sensitivity remained very low. For instance, the tested cisplatin $(300 \mu \mathrm{M})$ and cyclosporine A concentrations $(1 \mathrm{mM})$ exceeded therapeutic levels (200 nM and $300 \mathrm{nM}$ for cisplatin and cyclosporine A, respectively) by more than 1000 times. By contrast, using the same cell line in a plate design of two channels separated by a collagen gel, cisplatin, and cyclosporine A toxicity was reproducibly detected in a multicenter study at 5-15 $\mu \mathrm{M}$ and 5-30 $\mu \mathrm{M}$, respectively (Vormann et al., 2021). This is, however, in contrast to other studies culturing non-OAT1-overexpressing immortalized human PTECs in the same device in which cyclosporine A toxicity was not detected at $30 \mu \mathrm{M}$ (Vriend et al., 2020).

For cisplatin, disruption of barrier integrity, lactate dehydrogenase release, and apoptosis were observed at lower levels of $25-100 \mu \mathrm{M}$ when PTECs were cultured in a two-channel microfluidic device (Jang et al., 2013; Nieskens et al., 2020). By contrast, a bioprinted proximal tubule model comprised of fibroblasts, HUVEC cells, and primary human PTECs detected cisplatin toxicity at concentrations below $5 \mu \mathrm{M}$ (King et al., 2017).

Besides cisplatin, the P-gp substrate cyclosporine A has been repeatedly used to evaluate functiondependent nephrotoxicity. In a bioprinted 3D proximal tubule on chip, reduced viability and significant disruption of barrier function were detected at 50 $\mu \mathrm{M}$ (Homan et al., 2016). Sensitivity to cisplatin and cyclosporine A was even lower in perfused proximal tubule models based on HK-2 cells (Sakolish et al., 2019). Here, changes in viability were only detected at concentrations of $1.4 \mathrm{mM}$ cisplatin and $270 \mu \mathrm{M}$ cyclosporine A, and even at those levels, only mild elevations of the secreted toxicity markers NGAL and action potential (AP) were observed. In addition to cisplatin and cyclosporine $\mathrm{A}$, few studies have evaluated the nephrotoxicity of other prototypic toxins, such as polymyxin B (Weber et al., 2018) and aristolochic acid (Chang et al., 2017) in perfused proximal tubule models, and of tacrolimus in kidney organoids (Kim et al., 2020b). Although these studies are useful to elucidate the toxicity mechanisms of the respective compounds, larger screens are needed to conclude about the overall accuracy of organotypic kidney systems for the prediction of nephrotoxicity.

Only few studies have been presented that evaluated the nephrotoxicity of a larger set of compounds. In an important seminal study, the predictivity of $2 \mathrm{D}$ cultured PTECs was compared with HK-2 and LLCPK1 cells using a panel of 41 drugs and chemicals consisting of 22 nephrotoxins that injure the proximal tubule, 11 nephrotoxins that injure other parts of the nephron, and 8 drugs that are not nephrotoxic (Li et al., 2013). Using IL6 and IL8 expression as endpoints after 16 hours of treatment, the model achieved sensitivity and specificity between $60 \%$ and $80 \%$. The area under the receiver operating characteristic $\left(\mathrm{AUC}_{\mathrm{ROC}}\right)$ across three PTEC donors was 0.85 compared with 0.71 and 0.73 for HK-2 and LLCPK1 cells, respectively. When ESC-derived instead of primary proximal tubule cells were used for the same 41 compounds, $\mathrm{AUC}_{\mathrm{ROC}}$ was only slightly lower with 0.8 (Li et al., 2014). By contrast, evaluation of a subset of 30 compounds using iPSC-derived proximal tubule cells and random forest-based model training, the model predicted proximal tubular toxicity in humans with $99.8 \%$ in the training set and $87 \%$ in the test set using crossvalidation using changes in IL6 and IL8 expression as readout (Kandasamy et al., 2015).

Besides IL6 and IL8 expression, other studies used different biomarkers for nephrotoxicity detection. A small study using six nephrotoxins showed that protein levels of KIM-1 and NGAL in primary PTECs correlated well with toxicity, whereas viability or the same endpoints in HK-2 cells was not suitable (Huang et al., 2015). Similarly, clusterin levels in primary PTECs achieved $65 \%$ sensitivity and $94 \%$ specificity in a set of 20 DIKI-positive and 16 DIKI-negative test compounds (Bajaj et al., 2020). Multiplexed gene expression profiling of primary PTECs moreover identified heme oxygenase-1 (HO-1) protein deregulation as a significant biomarker for all nine tested nephrotoxic compounds (Adler et al., 2016). Importantly, using an additional panel of 39 mechanistically distinct nephrotoxins, $\mathrm{HO}-1$ achieved higher $\mathrm{AUC}_{\mathrm{ROC}}$ than conventional measures of cell viability $(0.89 \mathrm{com}-$ pared with 0.78 ). Although these in vitro findings align 
with earlier results showing that HO-1 was increased in urine of patients with acute kidney injury (Zager et al., 2012) and tubulointerstitial damage (Yokoyama et al., 2011), it remains to be determined whether HO1 is indeed superior in vivo compared with conventional biomarkers.

These data indicate that available test systems based on primary or stem cell-derived proximal tubule cells provide useful tools for preclinical nephrotoxicity assessments. However, the tested endpoints remain diverse, and integration of the best-performing markers promises to further increase the predictive power. Notably, to the best of our knowledge, all current test systems used conventional $2 \mathrm{D}$ cultures of proximal tubule cells. It will be interesting to see whether the increased complexity of 3D renal models, such as fluidic chips or organoids, will translate into increased predictive accuracy. Furthermore, whether models of other sites of kidney injury, such as the glomerulus, can be useful in reliably identifying nephrotoxic compounds remains to be determined.

\section{Intestine}

Oral drug delivery constitutes the most attractive form of drug administration due to its ease of administration, which facilitates patient compliance, and its compatibility with solid formulations that allow for controlled and sustained drug delivery. Importantly, however, the suitability of a drug for oral administrations relies on its efficient absorption in the gastrointestinal tract. As such, the iterative testing and optimization of oral bioavailability constitutes an important aspect of the preclinical drug development. Gastrointestinal absorption of drugs can occur via transcellular transport and paracellular transport of transcytosis. Transcellular transport can be furthermore subdivided into passive transcellular transport in which mostly lipophilic compounds diffuse through membranes along their concentration gradient and active transcellular transport in which drugs are absorbed via transporter proteins. Although the former is nonsaturable, ATP-independent, and not dependent on the enantiomeric or diastereomeric configuration of the molecule, transporter-mediated uptake can be saturated and is commonly stereoisomer-specific.

Paracellular transport constitutes another passive uptake route in which molecules and solutes diffuse through the intercellular space, thus entering the blood stream without passing through cellular membranes. The paracellular transport comprises two distinct mechanisms. The size- and charge-selective high-capacity pore pathway mediates the uptake of small hydrophilic molecules with diameters $<6 \AA$, whereas the leak pathway has low capacity, is saturable, and allows the flux of molecules with diameters up to $100 \AA$ (Choi et al., 2017). Pore pathway fluxes occur through pores in tight junctions, whereas leak pathway flux is believed to occur through dysfunctional cell junctions or tricellular contact sites (Krug et al., 2009; Marchiando et al., 2010). Lastly, transcytotic trafficking is receptor-mediated and is particularly relevant for the uptake of immunoglobulins and nanoparticles (Thuenauer et al., 2017; Reinholz et al., 2018).

To evaluate oral drug absorption during preclinical development, a multitude of in vitro (Billat et al., 2017; Youhanna and Lauschke, 2021) and in vivo models (Cheng et al., 2008) are available. Notably, animal models differ substantially from humans in intestinal physiology as well as expression patterns and substrate specificities of intestinal transporters (Kararli, 1995; Cao et al., 2006; Chu et al., 2013). Although dogs and monkeys are considered as

TABLE 4

Advantages and limitations of current intestinal in vitro culture systems

\begin{tabular}{|c|c|c|c|c|c|}
\hline & Transwell Cultures & Perfusion Systems & Ussing Chambers & Organoids & Explant Cultures \\
\hline Medium dynamics & Static & Perfused & Static & Static & Static \\
\hline $\begin{array}{l}\text { Available drug } \\
\text { permeability data }\end{array}$ & +++ & + & +++ & - & ++ \\
\hline Cellular complexity & $\begin{array}{l}\text { Enterocytes }+/- \\
\text { Goblet cells and M } \\
\text { cells }\end{array}$ & $\begin{array}{l}\text { Enterocytes, Goblet } \\
\text { cells, enteroendocrine } \\
\text { cells, Paneth cells }\end{array}$ & All intestinal cell types & $\begin{array}{l}\text { Enterocytes, Goblet } \\
\text { cells, enteroendocrine } \\
\text { cells, Paneth cells }\end{array}$ & $\begin{array}{c}\text { All intestinal cell } \\
\text { types }\end{array}$ \\
\hline Molecular phenotype & + & ++ & +++ & ++ & +++ \\
\hline Mucus & $\begin{array}{c}\text { Some, if Goblet cells } \\
\text { are added }\end{array}$ & $\begin{array}{c}\text { Can reach physiologic } \\
\text { levels }\end{array}$ & Yes & Some & Yes \\
\hline Phenotypic stability & Multiple weeks & Multiple weeks & Hours & $\begin{array}{c}\text { Multiple weeks, allow } \\
\text { passaging }\end{array}$ & $6-24 \mathrm{~h}$ \\
\hline Throughput & +++ & ++ & + & +++ & + \\
\hline Ease of use & +++ & + & ++ & + & ++ \\
\hline Costs & +++ & + & ++ & +++ & ++ \\
\hline Model versatility & ++ & +++ & + & +++ & ++ \\
\hline Microbiota & No & Yes & No & Yes & No \\
\hline Peristalsis & No & Yes & No & No & No \\
\hline Main applications & Drug absorption & Disease modeling & Drug absorption & $\begin{array}{c}\text { Intestinal } \\
\text { development, } \\
\text { regenerative medicine }\end{array}$ & $\begin{array}{l}\text { Drug absorption, } \\
\text { acute toxicity }\end{array}$ \\
\hline
\end{tabular}


yielding the most translatable results, these only predicted $54 \%$ and $73 \%$ of human oral absorption of 56 small-molecule drugs, respectively (Ward et al., 2005). Notably, other studies showed better predictivity in rats (Chiou and Barve, 1998), dogs (Chiou et al., 2000), and monkeys (Chiou and Buehler, 2002). Thus, to complement animal data, a multitude of intestinal in vitro models based on human cells have been developed (Table 4). Transwell cultures are the preferred models for the evaluation of intestinal permeability. Microfluidic chips are useful to model effects of the microbiota and inflammation on intestinal phenotypes and permeability but have not been extensively used for absorption studies. Lastly, intestinal organoids are primarily used for studies of intestinal development and regenerative medicine. Furthermore, Ussing chamber experiments of intestinal preparations and precision cut intestinal slices constitute short-lived ex vivo models of intestinal tissue for permeability and toxicity studies. However, as they only allow for short-term maintenance of intact tissue function, they are out of the scope of this review, and we refer the interested readers to recent comprehensive and critical reviews on these systems (Li et al., 2016b; Thomson et al., 2019). To address the drug discovery needs of pharmaceutical companies, gastrointestinal models should ideally be high-throughput-compatible, allow for selective high exposure at the apical surface, recapitulate enterocytemicrobiome-immune interactions, enable the measurement of functional endpoints, and be accessible to genetic manipulation to investigate toxicity mechanisms (Peters et al., 2020).

\section{A. Transwell Cultures}

Transwell cultures in which human intestinal cells are cultured on a porous filter support constitute the most established in vitro culture paradigm for drug absorption studies (Fig. 5A). These models use primarily the human colon adenocarcinoma cell line Caco-2. When cultured to confluency, these cells regain features of differentiated enterocytes, including polarization, tight junctions, and apical brush borders (Sambuy et al., 2005; Sun et al., 2008). With few exceptions, such as BCRP and OATPB, differentiated Caco-2 cells expressed levels of ABC and SLC transporters as well as phase I and phase II drug-metabolizing enzymes that resembled those found in intestinal biopsies (Englund et al., 2006; Meinl et al., 2008; Ölander et al., 2016). As a consequence of these features, Caco-2 transwell cultures correlate well with the fraction of absorbed drugs in human in vivo studies (Artursson and Karlsson, 1991; Rubas et al., 1993; Stewart et al., 1995). Notably, transwell cultures do not provide absolute absorption values but rather semiquantitative data in which the rank of compound permeability in a set of test substances closely correlates with in vivo data. Furthermore, Caco-2 transwell assays are highly variable between laboratories due to the use of different Caco-2 subclones and passage numbers as well as insufficient protocol standardization with regards to media and culture time (Walter and Kissel, 1995; Hayeshi et al., 2008; Lee et al., 2017c).

Across compound classes, excellent correlations between human in situ data and Caco-2 transwell cultures were observed for hydrophobic drugs that are absorbed via the passive transcellular pathway, such as naproxen, antipyrine, and metoprolol (Lennernäs et al., 1996). In contrast, permeability coefficients of the hydrophilic paracellular uptake drugs terbutaline and atenolol as well as of carrier-mediated transport (levodopa, leucine, and glucose) were 1-2 orders of magnitude lower in transwell cultures compared with in vivo. The reasons for the lower permeability in Caco-2 transwell cultures compared with intestine in situ are likely the lower permeability of tight junctions with fewer pores albeit of similar diameter as well as the reduced surface area of monolayers compared with the extensively folded architecture of the intestine in vivo (Artursson et al., 1993; Tanaka et al., 1995). As a result, associations between transwell culture and in vivo absorption data are typically steep and sigmoidal.

Strategies to increase the paracellular permeability of transwell cultures included the replacement of Caco-2 cells with other cell lines that form less tight epithelia. Examples of other cell lines used for transwell absorption studies include IEC-18 derived from rat ileal crypts, the rat intestinal epithelial cell line 2/4/A1, and the human goblet cell lines HT29-MTX. 2/4/A1 transwell monolayer cultures showed transepithelial electrical resistance (TEER) within the physiologic range $\left(50 \Omega * \mathrm{~cm}^{2}\right)$ and one order of magnitude lower than in Caco- 2 cultures $\left(500-1000 \Omega * \mathrm{~cm}^{2}\right)$ (Tavelin et al., 2003a). In alignment with these findings, 2/4/A1 cells were better predictors of the oral absorption of incompletely absorbed drugs compared with Caco-2 (Tavelin et al., 2003b). However, 2/4/A1 cells lacked expression of key transporters, such as P-gp, MRP2, and BCRP. Similarly, IEC-18 cells featured physiologic TEER $\left(50 \Omega * \mathrm{~cm}^{2}\right)$ and increased paracellular transport compared with Caco-2 cells (Versantvoort et al., 2002; Steensma et al., 2004). However, carrier-mediated transport was absent, and metabolic profiles of isoflavones were considerably different from in vivo patterns. These results suggest that IEC-18 and 2/4/A1 cells might be useful tools for evaluations of passive permeability, whereas they are not suitable for studies of active uptake or intestinal metabolism.

HT29 cells constitute an additional human colorectal adenocarcinoma cell line that has been used for absorption studies. Using methotrexate, this cell line was differentiated into HT29-MTX mucus-producing goblet-like cells. Notably, although these cells form equally tight barriers in monolayers compared with Caco-2 (500-1000 $\left.\Omega^{*} \mathrm{~cm}^{2}\right)$, coculture of both cell lines 
A

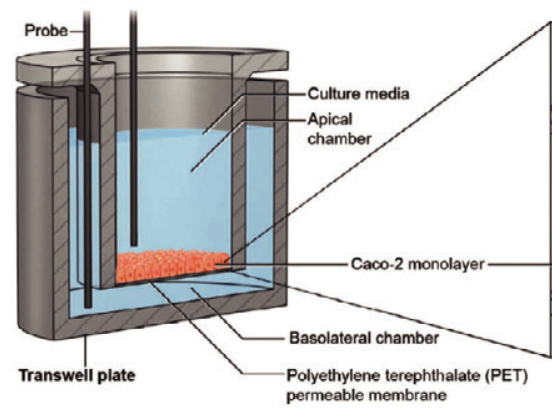

B

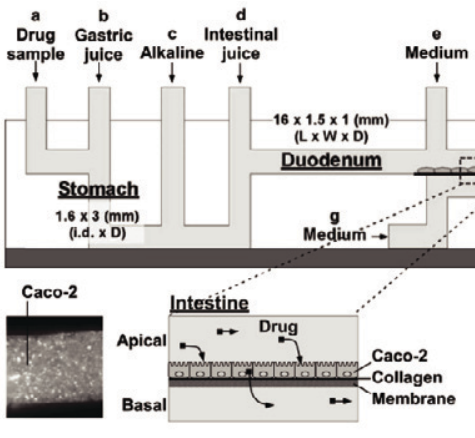

E

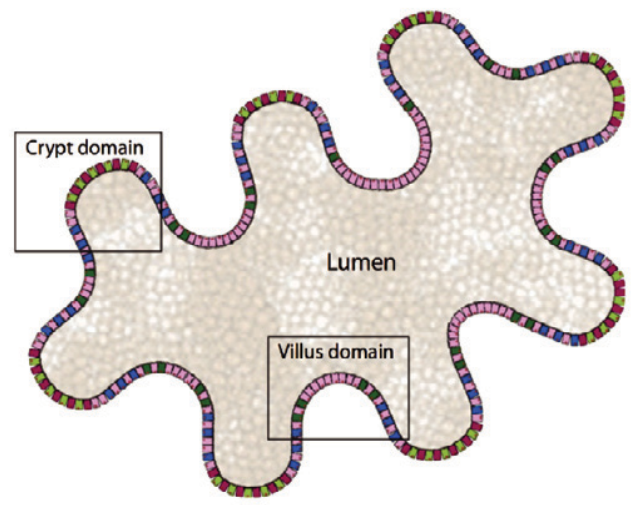

$\mathbf{F}$

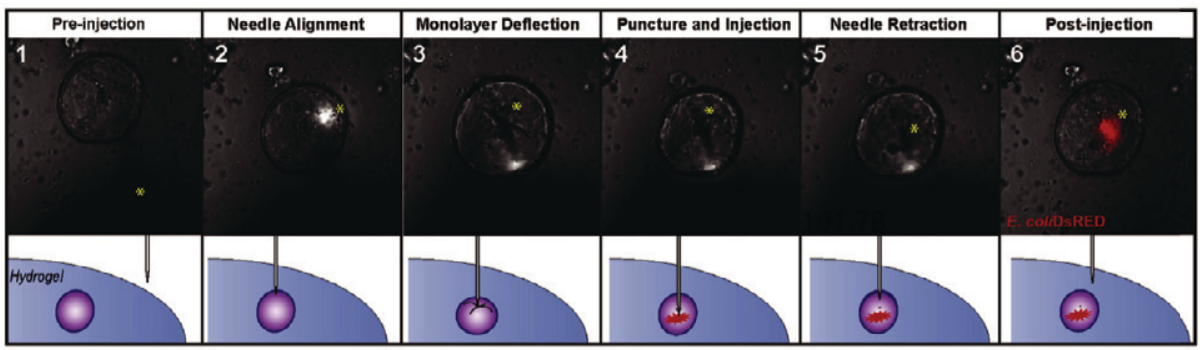

G

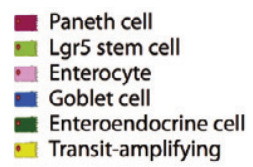

C

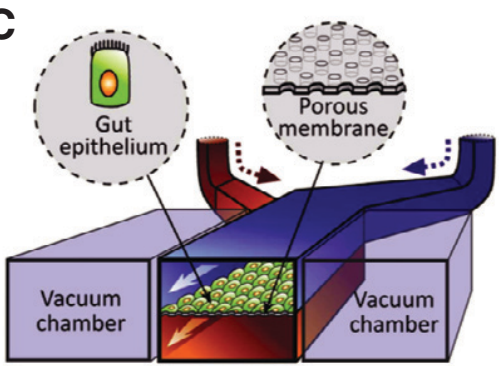

D Static (21 days) Phase contrast Occludin
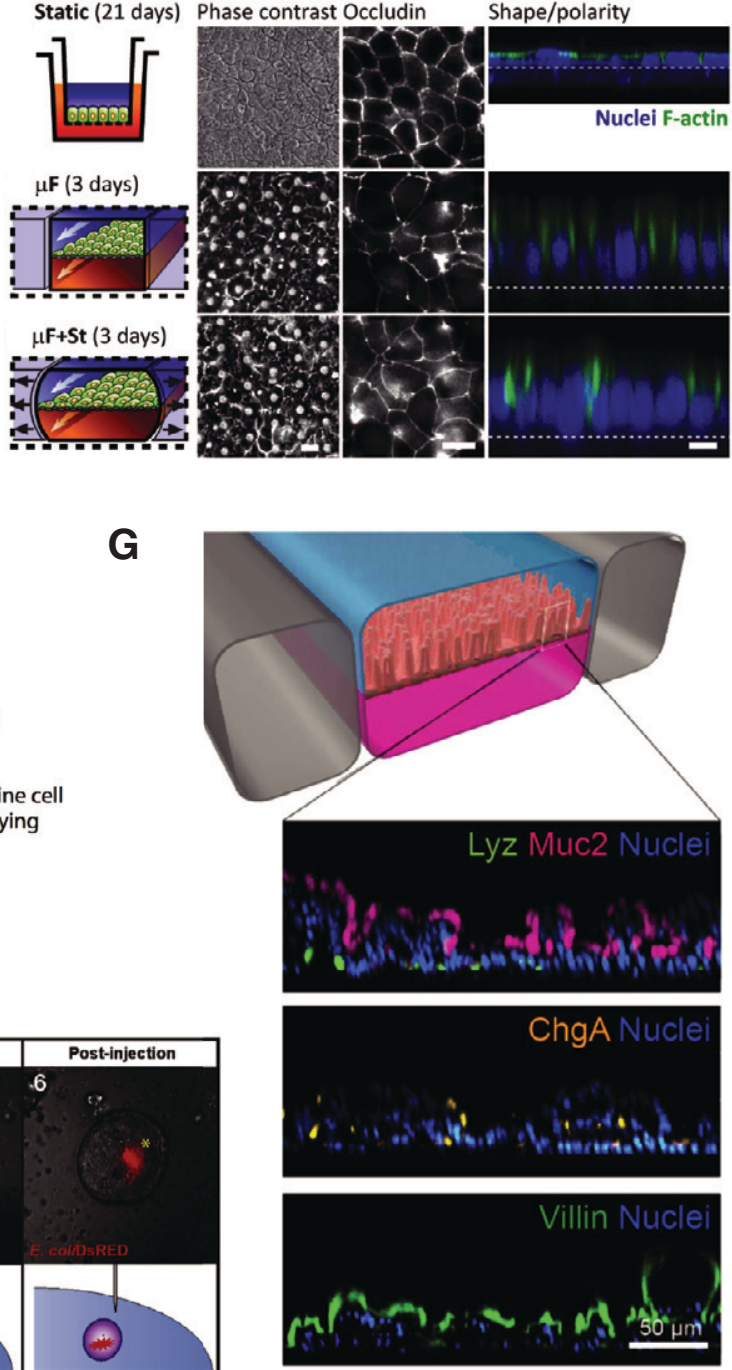

Fig. 5. Human intestinal 3D models for studies of drug permeability and disease modeling. (A) Schematic depiction of a static transwell culture. Caco2 cells are cultured as a monolayer on a porous membrane that separates the apical from the basolateral chamber. Measurement of TEER can be measured to evaluate barrier integrity. (B) Cross-sectional view of an integrated perfusion system for oral drug absorption. The drug of interest is first exposed to artificial gastric juice ( $\mathrm{pH}$ 2) before being neutralized and mixed with artificial intestinal juice containing bile acids in the duodenum. Subsequently, the solution was perfused past a Caco-2 transwell culture to mimic intestinal permeability. Compound molecules that permeated into the basal channel were exposed HepG2 cells to mimic first-pass metabolism before subsequently entering the target compartment, comprising in this case MCF7 breast cancer cells. (C) Schematic depiction of an intestinal microchip comprising a stretchable porous membrane that separates two independently perfusable microchannels, which are lined by vacuum chambers that allow the repeated application of mechanical strain mimicking intestinal peristalsis. (D) Shear stress due to microperfusion $(\mu \mathrm{F})$ and the application of cyclic mechanical strain $(\mu \mathrm{F}+\mathrm{St})$ increase cell height and polarization of Caco-2 monolayers while maintaining confluency as judged by localization of the tight junction protein occluding. Scale bars $=20 \mu \mathrm{m}$. (E) Intestinal organoids are comprised of villus domains containing enterocytes, enteroendocrine cells, and Goblet cells and alternating crypts enriched in LGR5-positive stem cells and Paneth cells. (F) Brightfield images (top row) and schematics (bottom row) of intestinal organoid injections using a customized robotic setup. For visualization purposes fluorescent DsRED-expressing $E$. coli are injected. Asterisk marks the needle tip. (G) Dissociated human intestinal organoids are seeded on the chip shown in (C and D). Immunofluorescence imaging shows expression of lysozyme (Lyz, Paneth cells), mucin 2 (Muc2, Goblet cells), chromogranin A (ChgA, enteroendocrine cells), and villin (cell apex). Figure modified with permission from (Imura et al., 2012; Kasendra et al., 2018; Kim et al., 2012; Robinson et al., 2019; Roeselers et al., 2013; Williamson et al., 2018). 
resulted in decreased TEER $\left(200 \Omega^{*} \mathrm{~cm}^{2}\right)$ and higher permeability for compounds transported via the paracellular pathway (Hilgendorf et al., 2000; Pontier et al., 2001). Coculture moreover led to increased apical-tobasolateral permeability, which was reduced in the opposite direction, consistent with the absence of Pgp expression in HT29-MTX cells. Further extension of the model by including lymphoblast-like Raji B cells as cell models of intestinal $\mathrm{M}$ cells resulted in a further reduction of TEER and increased absorption of hydrophilic compounds (markers of paracellular transport) (Bazes et al., 2011; Antunes et al., 2013; Araújo and Sarmento, 2013; Araújo et al., 2016). Furthermore, the transcellular transport of nanoparticles in triple cocultures closely correlated with uptake across porcine intestinal mucosa (Schimpel et al., 2014). Similarly, mimicking of the gastrointestinal immune compartment in a coculture of Caco-2 cells with human macrophages (THP-1) and dendritic cells (MUTZ-3) resulted in increased nanoparticle-mediated inflammatory responses and toxicity (Susewind et al., 2016). Combined, these results suggest that triple cocultures offer advantages over conventional cultures, as intestinal immune cells are central sites of nanoparticle transcytosis, and mucus produced by goblet-like cells can impact nanoparticle mobility.

Overall, static transwell culture systems thus present a well established platform for studies of drug permeability. Permeability coefficients of enterocyte-like cell monocultures correlate well with the absorbed drug fraction in humans in vivo and allow for easy distinction between completely and incompletely absorbed drugs. Coculture with goblet-like cells and intestinal immune cells moreover extends the utility of the model to predict paracellular transport and transcytosis. However, although transwell cultures are generally considered as the gold standard for in vitro drug absorption studies, interlaboratory variability is extensive, which has important implications for the direct use of permeability data.

\section{B. Perfused Intestinal Microchips}

Although static transwell cultures constitute reliable models for the prediction of intestinal permeability, they fall short of mimicking the complex physiology of the human intestine in vivo. Specifically, static transwell cultures lack intraluminal flow and peristaltic motions. Although a multitude of different approaches and devices have been presented, concepts are mostly similar in that intestinal cells (mostly Caco-2) are cultured on transwell membranes, which are positioned between two independently perfused microchannels, mimicking the luminal and serosal sides of the intestinal epithelium.

Early seminal approaches used devices made from PDMS with embedded stirrer-based micropumps and polyester semipermeable membranes on which Caco-2 cells were cultured to confluency (Kimura et al., 2008). The monolayers were intact for at least 3 weeks and allowed for online fluorescence-based pharmacokinetic measurements of rhodamine- 123 basolateral-to-apical transport. More refined systems for real-time measurements that use mass spectrometry instead of fluorescence as a readout for permeation of drugs through the intestinal barrier have been presented (Gao et al., 2013; Santbergen et al., 2020). Thereby, the system is compatible with the accurate and rapid quantification of permeability of any molecule with established quantification assays using as little as $6 \mu \mathrm{l}$ of sample.

In a different PDMS-based chip, the authors used polyethylene terephthalate membranes and syringe pumps instead of stirrer-based pumps for more precise flow control (Imura et al., 2009). This setup could distinguish compounds with high and low permeability, and benchmarking to conventional static transwell cultures did not result in significant differences. Interestingly, the system could furthermore be coupled to a series of upstream and downstream compartments (Imura et al., 2010, 2012). The upstream compartments contained buffers mimicking gastric and intestinal juices to mimic gastrointestinal digestion before entering the luminal channel of the intestinal culture, whereas the downstream channels can contain target cell models, such as liver or cancer cells to evaluate drug metabolism and efficacy, thus providing an integrated system for oral uptake studies (Fig. 5B).

Although early studies were mostly application-driven and benchmarked systems to transwell cultures, later approaches changed their focus to phenotypic analyses using the native intestine as comparator. In a remarkable setup that can be considered as the current gold standard of intestinal perfusion systems, the perfused transwell setup illustrated above was extended by the addition of two vacuum chambers adjacent to the membrane, which allowed for its rhythmic stretching, thus mimicking peristalsis (Fig. 5, C and D). Although flow and the resulting shear forces were sufficient to cause increases in cell height and an undulating morphology of the Caco-2 layer with higher pericellular permeability and the formation of microvilli-like structure, the addition of peristalsis caused a further increase in differentiation, as indicated by elevated expression levels of aminopeptidases (Kim et al., 2012; Pocock et al., 2017).

Strikingly, flow and peristalsis-like motions were moreover sufficient to reconstitute the cellular complexity of the intestinal epithelium (Kim and Ingber, 2013). After 3 weeks in culture, the seeded Caco-2 monolayer stained positive for markers of enterocytes, enteroendocrine, Goblet, and Paneth cells that were distributed across villi and crypts in alignment with their physiologic 
localization. Furthermore, EdU staining revealed proliferative centers almost exclusively in crypts, and the resulting daughter cells migrated upward along the villi structures. In addition to Caco-2 cells as cellular substrate, the model has been established using cells from human intestinal biopsies from macroscopically normal duodenums (Kasendra et al., 2018), iPS-derived intestinal organoids (Workman et al., 2017), or organoids from patients with Crohn disease, ulcerative, colitis or colorectal cancer (Shin et al., 2020). The use of primary patient-derived colonic epithelial cells allowed the reconstitution of mucus layers with physiologic thickness $(300-600 \mu \mathrm{m})$, which might be relevant for analyzing the effects of mucus on compound permeability and might protect cells from microbiota (Sontheimer-Phelps et al., 2020). Specifically, using an inoculum of complex gut microbiome derived from a human stool sample and maintained in gnotobiotic mice, a total of 200 operational taxonomic units from 11 different genera could be identified after 3 days in culture without compromising barrier integrity (Jalili-Firoozinezhad et al., 2019). Furthermore, phylum composition was sensitive to the oxygen concentration in culture and closely resembled composition in human stool. As a consequence, this platform might provide useful for the analysis of microbiome-drug interactions as well as discovery and development of probiotics.

Alternatively, the intestinal microarchitecture can be recapitulated by seeding cells onto hydrogel scaffolds that resemble the shape of intestinal villi. For instance, using poly-lactic-glycolic acid or poly-ethylene-co-vinyl-acetate scaffolds for the coculture of Caco-2 and HT29-MTX cells resulted in more physiologic TEER after 7 days $(<100$ $\Omega * \mathrm{~cm}^{2}$ ) compared with transwell cultures $\left(500 \Omega * \mathrm{~cm}^{2}\right)$, and application of flow resulted in a $>4$-fold increase in glucose uptake (Costello et al., 2014, 2017). Similarly, intestinal topography could be recapitulated using collagen and resulted in elevated CYP3A4 and aminopeptidase activity and enhanced mucus production as well as increased absorptive permeability (Sung et al., 2011; Kim et al., 2014; Shim et al., 2017; Wang et al., 2017c).

To further increase the physiologic relevance of culture, approaches have been presented in which human intestinal cells are cultured in ECM derived from porcine small intestine (Schweinlin et al., 2016). Specifically, primary intestinal crypts were isolated from healthy volunteers and, after 3-4 weeks of organoid culture, seeded onto the decellularized ECM scaffold in perfused transwell culture. As seen with other models, perfusion resulted in the differentiation of Goblet and enteroendocrine cells and increased CYP3A4 and transporter activity as well as physiologic TEER $\left(40 \quad \Omega^{*} \mathrm{~cm}^{2}\right)$. Furthermore, coculture with primary subepithelial fibroblasts isolated from connective tissue resulted in the formation of a heterogeneous bilayer in which fibroblasts were located underneath the epithelial layer. Alternative approaches using coculture of Caco-2 with primary human microvascular endothelial cells under perfused conditions resulted in increased transporter expression and higher permeability of lowly permeable compounds (Pusch et al., 2011).

To reduce effects of drug absorption, a membranefree model has been presented in which a perfused channel with Caco-2 cells is separated from an adjacent flow channel by an ECM gel made from rat collagen (Trietsch et al., 2017). The setup of the device is high-throughput compatible with 40 tubules on a single chip assessable by automated imaging. However, relatively high leakage frequency (7\%) and the lack of in-depth biologic or pharmacological characterization data in combination with undisclosed chip materials hamper its current use for toxicity or drug absorption studies. Alternatively, drug absorption can be reduced by using devices and membranes from lowly absorbing materials, such as Teflon, polymethyl methacrylate (PMMA), or thiol-ene (Tan et al., 2018).

\section{Intestinal Organoid Cultures}

Intestinal organoids constitute the first organoid cultures to be developed. In this culture paradigm, iPSCs (Spence et al., 2011; Takahashi et al., 2018) or LGR5+ primary stem cells isolated from intestinal crypts (Ootani et al., 2009; Sato et al., 2009) self-organize into 3D structures that recapitulate the cellular composition and structure of the intestinal epithelium (Fig. 5E). iPSCderived intestinal cultures are referred to as organoids, whereas adult stem cell-derived cultures are referred to as enteroids or colonoids, depending on their origin along the gastrointestinal tract. Importantly, iPSC-derived organoids resemble fetal intestine, whereas enteroids and colonoids derived from adult stem cells acquire more mature features (Finkbeiner et al., 2015; Aurora and Spence, 2016). Consequently, adult stem cell-derived enteroids have become the predominant model for most pharmacological and toxicological applications.

Upon embedding in matrigel (Sato et al., 2009; Gracz et al., 2015), collagen gels (Jabaji et al., 2013; Pastuła et al., 2016), or synthetic PEG-based hydrogels (Gjorevski et al., 2016; Cruz-Acuña et al., 2017), single LGR5+ cells proliferate and differentiate in the absence of any mesenchymal supporting cells and reconstitute the regional identity of the origin of the stem cell (Zachos et al., 2016). To support the development of intestinal organoids, medium is typically supplemented with EGF, Noggin, Wnt3a, and R-spondin (Holmberg et al., 2017). However, small molecule-based approaches that replace Wnt-3a with Chir99021 (Langlands et al., 2018; Li et al., 2018b), Noggin with LDN-193189 (Liu et al., 2018b), and R-spondin with RS-246204 (Nam et al., 2017) have been presented. Single cells first grow into 
cyst-like aggregates, which upon prolonged culture assume a budding-like morphology. Stem cells and Paneth cells can be found in crypt-like domains, whereas the epithelium lining the central lumen is comprised of enterocytes, enteroendocrine cells, and Goblet cells (Fig. 5E). Importantly, these organoids offer considerable advantages over other methodologies in that they are long-term stable, can be expanded virtually unlimitedly, and are compatible with cryopreservation. However, they also pose major limitations, particularly for pharmacological and toxicological applications, as their luminal side is not directly accessible, which complicates drug exposures mimicking oral administration and inoculation with microbiome.

To overcome these limitations, microinjection methodologies have been developed in which customized needles and semi-automated microinjection technologies were used to enable high-content sampling of several microinjected cargos (Williamson et al., 2018). Specifically, the authors showed reproducible injections of $0.2 \mathrm{nl}$ into individual organoids and demonstrated organoid barrier function using $70 \mathrm{kDa}$ dextran as well as microbiota survival and growth dynamics (Fig. 5F). However, despite the sophisticated setup, injection rates remained relatively low with approximately 90 organoids/h.

Organoid, enteroid, or colonoid cultures can also be dissociated after differentiation and can be seeded as monolayers on gelatin (Moon et al., 2014), collagen (Scott et al., 2016), matrigel (Kozuka et al., 2017), or a layer of feeder cells (Moorefield et al., 2018). Crypts can be directly cultured in monolayers, thereby bypassing the initial organoid step (Thorne et al., 2018). Interestingly, the resulting $2 \mathrm{D}$ cultures retain their cellular complexity and compartmentalize into different functional domains with proliferating cells at the edges and differentiated cells at the center of the monolayer cultures (Wang et al., 2017b). These organoid-derived monolayers provide an interesting interface with microfluidic technology. As illustrated above, dissociated human iPSC-derived organoids (Workman et al., 2017), enteroids (Schweinlin et al., 2016; Kasendra et al., 2018) or colonoids (Sontheimer-Phelps et al., 2020) can be cultured in perfused transwell culture, which allows for the combination of physiomechanic stimuli, such as flow and peristalsis, with the unrivalled cellular complexity in organoid culture (Fig. 5G). Furthermore, when seeding only the EPCAM + epithelial cells after dissociation and seeding those on transwell plates, the resulting monolayers showed high TEER $\left(350 \Omega^{*} \mathrm{~cm}^{2}\right)$ and relevant CYP3A metabolic activity, opening applications of organoids for pharmacokinetic studies (Yoshida et al., 2020).

\section{Disease Models}

The cellular complexity of advanced organotypic intestinal cultures and their compatibility with the sustained coculture of complex microbial communities opens exciting possibilities for the modeling of intestinal diseases. Most prominently, microfluidic chips and organoids have been used to study inflammatory bowel disease (IBD) and enteric viral infections.

To mimic intestinal inflammation, intestinal cultures are subjected to an immune-relevant inflammatory trigger. This can be achieved extrinsically by adding cocktails of cytokines, such as IL1 $\beta$, tumor necrosis factor- $\alpha$, and interferon $\gamma$ (Beaurivage et al., 2019; Gijzen et al., 2020), or by coculture with peripheral blood mononuclear cells (Kim et al., 2016a; Beaurivage et al., 2020). Upon monocyte activation using lipopolysaccharide from pathogenic Escherichia coli, Caco-2 cells were induced to secrete proinflammatory cytokines, which in turn compromise barrier integrity, thus mimicking aspects of IBD pathology. Furthermore, proinflammatory cues can facilitate the recruitment of neutrophils, which further worsens intestinal injury (Gjorevski et al., 2020). Exposure of a microfluidic model with microbiome and peristalsis with dextran sodium sulfate (DSS) could reproduce the disruption of barrier integrity and villus architecture observed in the corresponding colitis mouse model (Shin and Kim, 2018). Importantly, the authors found that administration of probiotics before DSS exposure could maintain the intestinal barrier, whereas the beneficial effect was lost when the bacteria were added after DSS. In a coculture with circulating Treg and Th17 cells, microbiome-derived short-chain fatty acids reduced immune cell activation while exacerbating activation of CD4+ T cells, which in turn resulted in barrier disruption (Trapecar et al., 2020).

Colonoids can be derived from routine biopsies of patients with IBD, thus offering possibilities to study patient-specific disease mechanisms. When compared with controls, organoids derived from patients with IBD exhibited an inflammatory phenotype with decreased size and budding capacity, increased cell death, and luminal debris, which could be curbed by treatment with the clinically approved IBD drugs methylprednisolone and mesalazine (d'Aldebert et al., 2020). In an interesting study, 76 clonal human colonoids were established from single cells isolated from inflamed or uninflamed of ulcerative colitis patients and controls (Nanki et al., 2020). Using whole-exome sequencing, the authors could replicate an increased burden of somatic mutations in genes related to IL-17 signaling in organoids derived from the affected epithelium compared with controls, thus providing a potential link between inflammation and oncogenesis.

In addition to IBD, intestinal cultures have been used to emulate a range of enteric infections. Intestinal microchip cultures could model coxsackie B1 virus infections and were able to emulate the entire virus life cycle from infection to replication and shedding of new virions (Villenave et al., 2017). In addition, peristalsis and organotypic architecture of the intestinal chips promoted infectivity of the enteroinvasive 
bacterium Shigella flexneri (Grassart et al., 2019). Pathogen adhesion was promoted around 10-fold, as the S. flexneri adhered specifically to the crypt-like domains. Moreover, mechanical stretching increased infectivity approximately 100-fold, suggesting that apical invasion strongly depends on peristaltic motions. Besides chip cultures, viral and bacterial infections have been studied extensively in intestinal organoids, such as norovirus (Ettayebi et al., 2016), adenovirus (Holly and Smith, 2018), SARS-CoV-2 (Krüger et al., 2020; Lamers et al., 2020; Zhou et al., 2020), Cryptosporidium parvum (Heo et al., 2018), Clostridium difficile (Leslie et al., 2015), and various pathogenic $E$. coli strains (In et al., 2016; Noel et al., 2017; Rajan et al., 2018). Furthermore, intestinal organoids have been instrumental in demonstrating a link between $E$. coli-encoding colibactin, increased somatic mutation rates, and colorectal cancer risk (Pleguezuelos-Manzano et al., 2020). These findings show that microphysiological systems can recapitulate the complexity of IBD and enteric infections and provide useful tools for the study of underlying disease mechanisms and the identification of immunomodulators and nutraceuticals as modulators of disease pathogenesis.

\section{Brain}

Constructing and deconstructing the complexity of the human brain is a particularly challenging task. Not surprisingly, most of our knowledge regarding the human brain comes from studies performed in the lissencephalic brains of rodents, observations in postmortem brain tissue, or noninvasive neuroimaging studies. Although such studies have provided insight into the fundamental principles of brain organization, at a mechanistic level, the diverse and intricate functions of the human brain still remain largely elusive. Studies in nonhuman primates may to some extent circumvent such limitations but are instead restricted by ethical considerations and the lack of scalability. The inaccessibility of the human brain also limits the use of primary in vitro cultures or primary in vitro organotypic brain slice cultures as cell models for pharmacological and toxicological testing.

Thus, the differentiation of human ESCs or reprogrammed iPSCs into neural-like cells has become a valuable tool as translational models of model brain disorders (Table 5). Early efforts to derive neurons in monolayer (2D) from ESCs and iPSCs have rapidly been complemented by a plethora of protocols for $2 \mathrm{D}$ and $3 \mathrm{D}$ generation of different neuronal subtypes as well as glia cells. Although such models are still a very active area of research and need to be critically evaluated, they hold obvious advantages, such as the possibility to model risk variants in the appropriate genomic landscape (i.e., by using patient-derived cells) as well as being compatible with a large range of experimental approaches, including genetic engineering, highly parallelized single-cell transcriptomics, and xenotransplantation.

\section{A. Deriving Brain Cells from Pluripotent Stem Cells}

ESCs are pluripotent stem cells derived from the inner cell mass of human blastocysts and can give rise to somatic cells of all three germ layers. In 2001, Zhang and colleagues (2001) showed that it was possible to enrich neuroepithelial cells from ESCs grown in vitro and further differentiate these cells to neuron- and macroglia-like cells. Detached ESC colonies were grown as free-floating cell aggregates in suspension, referred to as embryoid bodies, and under continuous exposure to FGF2 gave rise to rosettes that resembled the early neural tube and expressed typical neuroepithelial markers. After withdrawal of FGF2, these cells then differentiated to neurons and, to a lesser extent, astrocytes and oligodendrocytes. With the discovery in 2007 that pluripotent stem cells can also be derived from somatic cells by direct reprograming (Takahashi et al., 2007), this opened up possibilities for patient-specific modeling and spurred efforts to further optimize culture conditions for neural in vitro differentiation. A few years later, it was established that two inhibitors of SMAD signaling, Noggin and SB431542, were sufficient to induce robust neural conversion of ESCs, thus bypassing embryoid body formation (Chambers et al., 2009). A plethora of protocols have since emerged to efficiently derive cells of various neuronal subtypes (Tao and Zhang, 2016) as well as astrocytes (Krencik et al., 2011; Canals et al., 2018), oligodendrocytes (Hu et al., 2009; Song et al., 2012b; Wang et al., 2013; Douvaras et al., 2014), and microglia (Muffat et al., 2016; Abud et al., 2017; Haenseler et al., 2017; Pandya et al., 2017). In parallel, approaches for direct conversion of easily accessible somatic cells into neurons (Yang et al., 2011; Pereira et al., 2014), astrocytes (Caiazzo et al., 2015), oligodendrocytes (Pouya et al., 2020; Yavarpour-Bali et al., 2020), and microglia (Sellgren et al., 2017) have been presented that offer alternatives to reprogramming through iPSCs. In contrast, the culture of functional primary neural cells remains difficult and, with few recent exceptions (Zhang et al., 2016a; Spaethling et al., 2017; Park et al., 2020), is limited to peripheral nerves of animal species.

\section{B. Organotypic and Microphysiological Human $3 D$ Tissue Models of the Brain}

Cerebral organoids, or brain-region-specific organoids, ultimately rely on the self-organizing capacity of pluripotent stem cells in 3D aggregates to recapitulate tissue architecture. To what extent the models are optimized regarding environmental conditions to support intrinsic cues versus the addition of external inductive signals to instruct the cells toward an ectodermal fate defines the two major approaches: undirected and directed organoid differentiation (Fig. 6). 
TABLE 5

Overview of organotypic tissue cultures for cerebral disease modeling

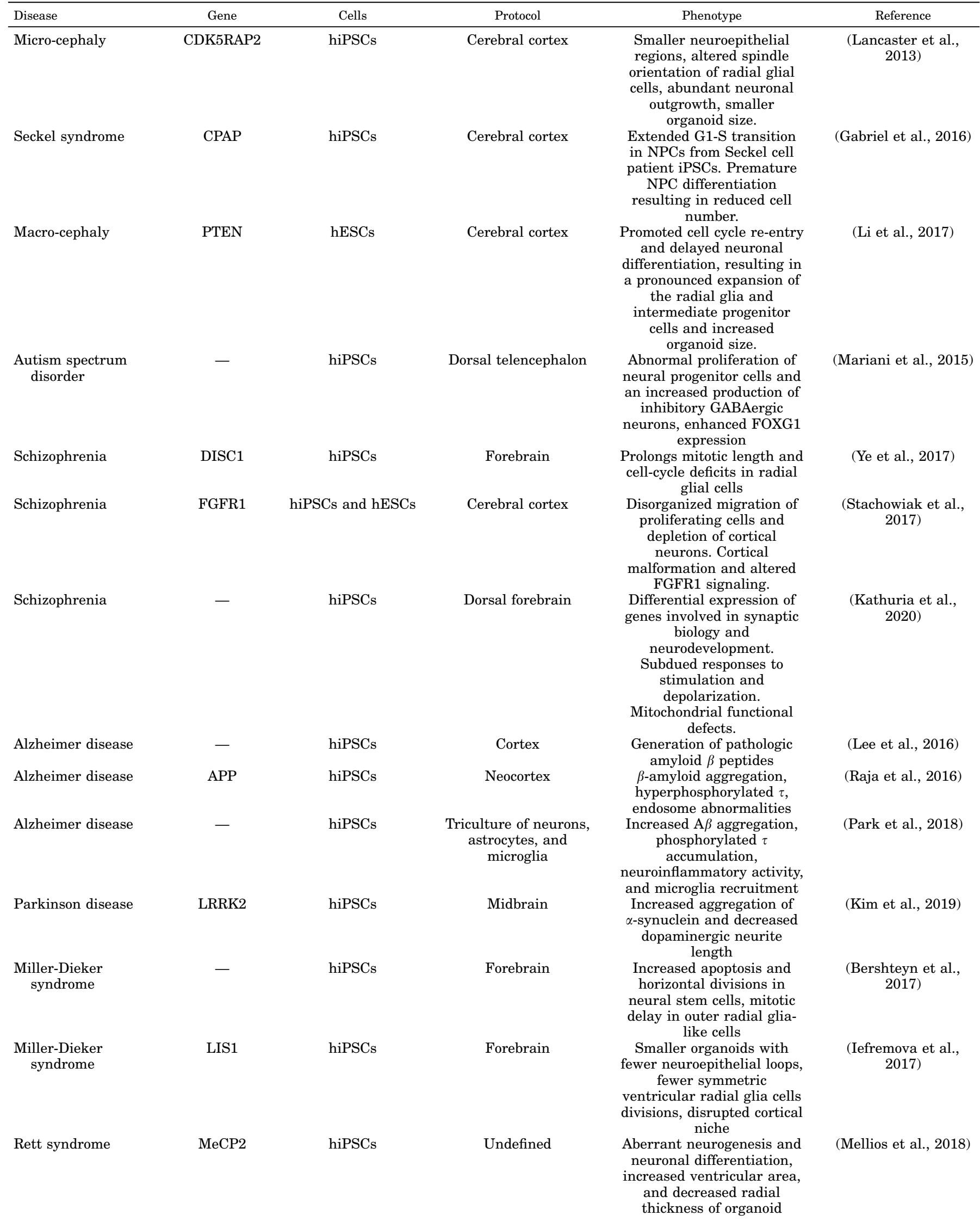




\begin{tabular}{|c|c|c|c|c|c|}
\hline Disease & Gene & Cells & $\begin{array}{c}\text { LE 5-Continued } \\
\text { Protocol }\end{array}$ & Phenotype & Reference \\
\hline ZIKA virus infection & ZIKV & hiPSCs & Forebrain & $\begin{array}{c}\text { Suppression of neural } \\
\text { progenitor cell } \\
\text { proliferation, decreased } \\
\text { neuronal layer thickness } \\
\text { and organoid size, enlarged } \\
\text { lumen/ventricles }\end{array}$ & (Qian et al., 2016) \\
\hline ZIKA virus infection & ZIKV & hESCs & Cerebral cortex & $\begin{array}{l}\text { ZIKV-mediated TLR3 } \\
\text { activation, reduction in } \\
\text { organoid volume } \\
\text { resembling microcephaly. }\end{array}$ & (Dang et al., 2016) \\
\hline ZIKA virus infection & ZIKV-NS2A & hiPSCs & Forebrain & $\begin{array}{l}\text { ZIKV-NS2A impairs radial } \\
\text { glial cell proliferation and } \\
\text { adherens junction } \\
\text { formation }\end{array}$ & (Yoon et al., 2017) \\
\hline
\end{tabular}

hESC, human embryonic stem cell.

In addition, microfluidic "brain-on-a-chip" or "bloodbrain-barrier (BBB)-on-a-chip" systems have been developed and proven useful in drug discovery and assessing drug delivery and toxicity.

1. Undirected Cerebral Organoids. In 2013, neuroectoderm of embryoid bodies was first maintained in 3D culture embedded in matrigel (Lancaster et al., 2013; Lancaster and Knoblich, 2014). The droplets were then transferred to a bioreactor to ensure adequate nutritional supply and kept in culture for up to 10 months. Immunohistochemistry revealed that these $3 \mathrm{D}$ cultures usually referred to as cerebral organoids or undirected whole-brain organoids displayed a cellular diversity and architecture that partly recapitulate the structure of the brain as early as 20-30 days after the start of culture. The self-organizing capacity was later confirmed by single-cell transcriptomic analyses that revealed a mixture of region specific ectodermal and mesodermal cells. Upon graftment of such undirected human cerebral organoids into the adult mouse brain, the models established graft-to- host functional synaptic connectivity and became vascularized (Mansour et al., 2018). Due to significant organoid-to-organoid variability, modifications of the original protocol have been proposed to support the formation of neuroectoderm (e.g., by using a microscale internal scaffold or small molecules or by decreasing the size of the embryoid bodies) (Lancaster et al., 2017; Sivitilli et al., 2020). On the contrary, a recent study increased the percentage of mesodermal progenitors, by lowering the heparin concentration and delaying matrigel embedding, and observed innately developing microglia-like cells (Ormel et al., 2018). However, this strategy likely entails increased organoid-to-organoid variability, and single-cell transcriptomic analyses are warranted to evaluate the effects on cell composition.

2. Directed Cerebral Organoids. In contrast to undirected methods, directed protocols rely to a higher degree on the addition of external patterning factors, such as small molecules and morphogens, to achieve the development of patterned organoids with region-specific neural cell types from ESCs and iPSCs

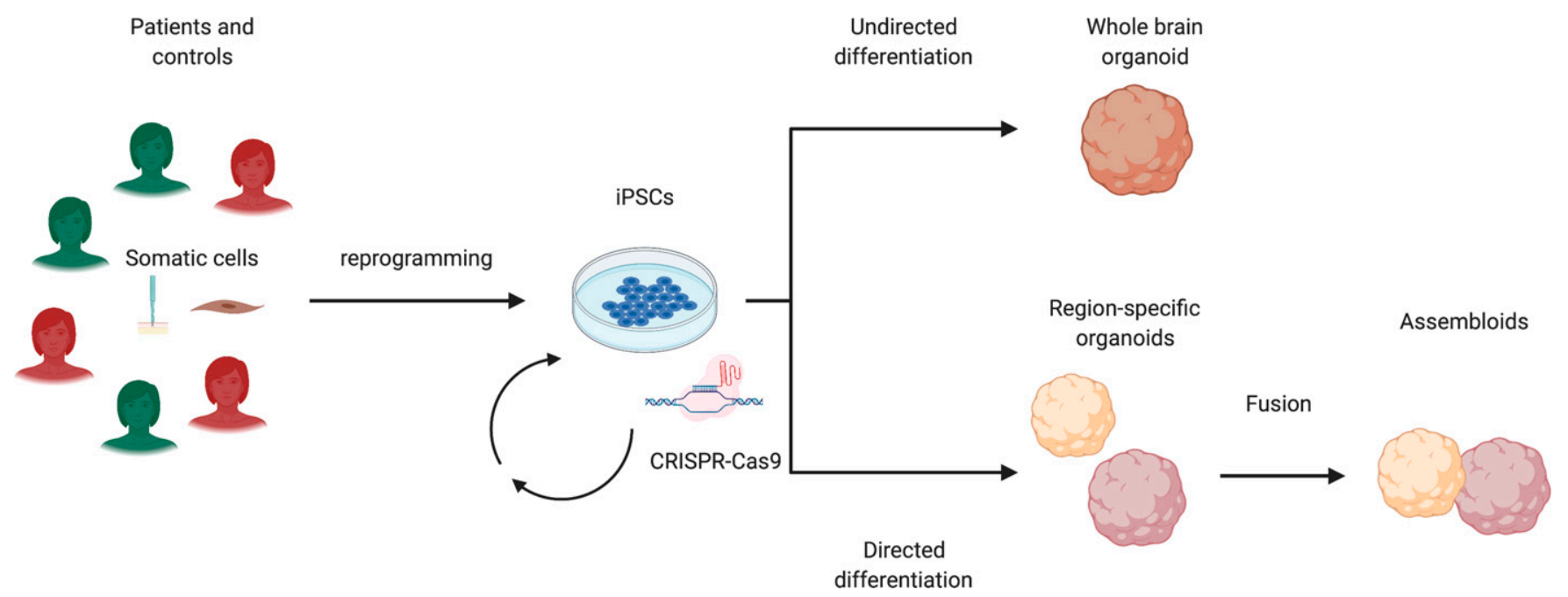

Fig. 6. Approaches for generating cerebral organoids for disease modeling. Easily accessible somatic cells from patients and controls can be reprogrammed into iPSCs that can be manipulated by genetic engineering to create isogenic lines. iPSCs can then be used to create either whole brain organoids through undirected differentiation or to region-specific organoids through patterned or directed differentiation. Region-specific organoids can moreover be fused to create so-called assembloids to recapitulate connectivity. 
(Eiraku et al., 2008; Kadoshima et al., 2013). Subsequently, a number of different protocols have been reported to generate region-specific patterned organoids of the cortex (Mariani et al., 2015; Paşca et al., 2015; Lancaster et al., 2017; Qian et al., 2017a, 2018), midbrain (Jo et al., 2016; Qian et al., 2016), cerebellum (Muguruma et al., 2015), pituitary gland (Ozone et al., 2016), and hypothalamus (Qian et al., 2016). In an interesting strategy, these region-specific organoids can be fused into so called "assembloids" that to some extent enable modeling of functional connectivity (Birey et al., 2017).

3. Neural Spheroids. In addition to complex and heterogeneous stem cell-derived organoids, spheroid cultures have been presented in which differentiated cells are aggregated in engineered scaffolds or in concave microwell plates. Although these models have clear advantages over conventional $2 \mathrm{D}$ culture, they lack the cellular complexity that can be achieved with organoids and do not recapitulate the tissue architecture of the human brain. Most 3D neural spheroids have used alginate or collagen scaffolds ( $\mathrm{Li}$ et al., 2007; Xu et al., 2009; Frampton et al., 2011; Odawara et al., 2013; Tang-Schomer et al., 2014). However, more recently scaffold-free methods have emerged (Choi et al., 2013; KatoNegishi et al., 2013). One promising example is the formation of spheroids using primary human astrocytes, pericytes, and endothelial cells, in which these cell types form layered structures with a core of astrocytes surrounded by pericytes and endothelial cells, thus recapitulating important structural BBB features (Urich et al., 2013).

4. Microfluidic Brain-on-a-Chip Models. Brain-ona-chip models comprise microengineered systems that create opportunities for spatiotemporal control of cell growth and differentiation cues by combining surfaces mimicking the ECM with microfluidic channels regulating the mass transport of fluids and soluble factors. These chips can be manufactured in a variety of material, of which PDMS remains most commonly used for brain-on-a-chip devices (Mofazzal Jahromi et al., 2019). BBB microchip models frequently based on conventional transwell cultures in which a porous barrier membrane coated with endothelial cells, pericytes, and astrocytes separates two adjacent compartments. Notably, the two different compartments are commonly perfused using different media resembling blood and cerebrospinal fluid. Such perfusion devices improve the stability of $\mathrm{pH}$ and oxygen in the media compared with static cultures in which media were exchanged periodically. Seminal studies presented a PDMS device with a vascular compartment containing primary endothelial cells separated from a neural chamber containing primary human astrocytes and pericytes as well as iPSC-derived glutamatergic neurons by a porous polycarbonate membrane (Brown et al., 2015; van der Helm et al., 2016). Cells remained viable for up to 21 days and displayed relevant expression of the tight junction marker ZO-1.

The two compartment chips were recently extended to include an additional compartment so that $\mathrm{BBB}$ influx, brain parenchyma, and BBB efflux could be modeled simultaneously (Maoz et al., 2018). The brain compartment consists of stem cell-derived glutamatergic, GABAergic, dopaminergic, and serotonergic neurons as well as glial cells, whereas the BBB compartments each consist of a basal channel lined with primary endothelial cells separated from an apical, perivascular channel by a porous membrane coated with astrocytes and pericytes. Shear stress on astrocytes and pericytes was $>25$-fold lower than in the endothelial compartment $\left(0.0007\right.$ vs. 0.02 dyne $\left./ \mathrm{cm}^{2}\right)$, closely mimicking the native microenvironment. By restricting active flow to the apical channel of the brain chip, flow velocity neural compartment approached zero, causing diffusion-mediated molecular transport to dominate, as previously reported for the brain in vivo (Ballabh et al., 2004). The model could mimic the effect of intravascular administration of the psychoactive drug methamphetamine on the reversible breakdown of the BBB observed in vivo (Turowski and Kenny, 2015) and revealed previously unknown metabolic coupling between the BBB and neurons, offering an in vitro platform for probing transport, efficacy, mechanism of action, and toxicity of neuroactive drugs.

Other BBB models are designed so that the endothelium forms microvessels, which are in direct contact with astrocytes and pericytes without an artificial membrane (Herland et al., 2016). Stimulation with tumor necrosis factor- $\alpha$ resulted in significantly increased endothelial secretion of G-CSF and IL6 compared with static transwell cultures, emphasizing the importance of direct cell-cell crosstalk and perfusion for proper mimicking of human BBB biology and neuroinflammation.

Several microfluidic brain-on-a-chip as well as BBB-on-a-chip devices have been applied to study neural toxicity and $\mathrm{BBB}$ permeability as well as to identify novel drug target candidates for the treatment of brain diseases. Although these systems have obvious advantages in terms of scalability and control of the microenvironment, it is important to consider that the reversed-engineering approach remains hampered by our incomplete understanding of brain ontogenesis and physiology.

\section{Applications of Organotypic Brain Models}

1. Drug Screening Platforms. Organotypic and microphysiological human brain models are extensively used for studies of BBB drug permeability. Using spheroids of primary human astrocytes, pericytes, and endothelial cells, drug penetration could be studied by confocal microscopy or mass spectrometry. Specifically, analyses revealed that the BBB-permeable 
compounds angiopep-2 and BKM120 passed the BBB, whereas the non-BBB-permeable tyrosine kinase inhibitor dabrafenib was not detected within spheroids (Bergmann et al., 2018), rendering this model suitable for cost-effective high-throughput drug permeability testing (Cho et al., 2017). The model system was recently extended to also include iPSC-derived microglia, oligodendrocytes, and neurons (Nzou et al., 2018). Although spheroids expressed cell-specific markers and functional barrier assessments using MPTP, $\mathrm{MPP}+$, and mercury chloride indicated charge selectivity, further characterizations of ECM structure and composition as well as functional analyses of the individual cell types in the model are necessary to better understand its physiologic relevance.

Such 3D systems have obvious advantages in comparison with $2 \mathrm{D}$ models regarding drug screening. However, limitations concerning scalability and intraexperimental and interexperimental reproducibility have stalled their adoption as high-throughput assays for drug development, particularly for models that use primary human cells. To overcome these limitations, a fully automated workflow was developed that provides an integrated solution for the generation, maintenance, and high-content imaging of human midbrain organoids in 96-well format (Renner et al., 2020). The authors demonstrate that the formed organoids recapitulate midbrain structures and display reduced morphologic, structural, and functional heterogeneity, thus providing a promising platform for future drug screening efforts. Similarly, high-throughput generation of homogeneous cerebral organoids derived from iPSCs of patients with spontaneous Alzheimer disease facilitated the testing of FDAapproved drugs as repurposing candidates to reduce amyloid $\beta(\mathrm{A} \beta)$ or $\tau$ deposition and enhance neuronal cell viability (Park et al., 2021).

In an interesting approach, ESC-derived neural progenitor cells, endothelial cells, mesenchymal stem cells, and microglia precursor cells were integrated in hydrogels (Schwartz et al., 2015). The resulting 3D neural constructs exhibited reproducible RNA sequencing gene expression signatures, and training with 34 toxic and 26 nontoxic chemicals yielded a linear support vector model that correctly classified 9 out of $10 \mathrm{com}$ pounds from an additional blinded test set as toxic or nontoxic, respectively, with one false positive.

A further interesting application is the use of brain organoids with clinically relevant oncogenic mutations introduced by CRISPR/Cas for the development of targeted treatments for brain tumors (Bian et al., 2018). As a proof of concept, the authors found that only those organoids with mutations resulting in EGFR overactivation were responsive to the EGFR inhibitors. Furthermore, human cerebral organoids and brain-on-a-chip systems have been used in several studies to evaluate effects of environmental exposures, such as ethanol (Zhu et al., 2017), nicotine (Wang et al., 2018b), and cocaine (Lee et al., 2017a), on prenatal neurogenesis.

2. Disease Models. Organotypic and microfluidic 3D models of the human brain have in recent years been widely used to model CNS pathologies using patient-derived iPSCs or genomic editing in which the effect of genetic risk variants can be directly compared with isogenic control lines. Both undirected and directed differentiation protocols as well as spheroids and microfluidic systems have been used to assess a growing number of diverse neural pathologies, including monogenic diseases, complex neurodegenerative disorders, brain tumors, and neurotropic infectious diseases, which facilitates the understanding of underlying disease mechanisms and enables subsequent drug discovery studies.

Undirected organoids were successfully used to recapitulate autosomal recessive primary microcephaly due to nonsense mutations in CDK5RAP2, which resulted in premature neuronal differentiation and smaller overall organoid size as compared with controls (Lancaster et al., 2013). Similarly, undirected cerebral organoids could mimic phenotypes associated with Sandhoff disease, an infantile $\mathrm{G}_{\mathrm{M} 2}$ gangliosidosis caused by mutations in HEXB that results in a deficiency of the lysosomal enzyme $\beta$ hexosaminidase and early ganglioside accumulation in the brain (Allende et al., 2018). Specifically, organoids generated from patient-derived iPSCs recapitulated GM2 ganglioside accumulation, which could be rescued by CRISPR/Cas of the underlying mutation. Furthermore, directed midbrain organoids with the Parkinson disease-associated LRRK2 mutation recapitulated several pathologic hallmarks of LRRK2associated sporadic Parkinson disease (Kim et al., 2019).

Brain organoids have moreover been used to model polygenic diseases. Transcriptomic comparison of dorsal/ventral forebrain organoids from members of four families with severe idiopathic autism spectrum disorder (ASD) or unaffected, first-degree family members revealed an upregulation of genes involved in cell proliferation, neuronal differentiation, and synaptic functions as well as an overproduction of GABAergic inhibitory neurons in affected organoids compared with controls (Mariani et al., 2015). Importantly, by downregulating FOXG1, which was upregulated in ASD organoids, the overproduction of GABAergic neurons could be reversed, suggesting the FOXG1 axis as an interesting target for the treatment of ASD.

Organotypic or microfluidic 3D models have also been used to study a variety of neurodegenerative disorders, such as Alzheimer disease, Parkinson disease, and amyotrophic lateral sclerosis. Using a microfluidic $3 \mathrm{D}$ triculture system consisting of neurons and astrocytes derived from human neural progenitor cells 
together with SV40 immortalized microglia, overexpression of a human $\beta$-amyloid precursor variant resulted in $\mathrm{A} \beta$ aggregation and increased microglial recruitment, which entailed secretion of proinflammatory cytokines as well as neuron loss (Park et al., 2018). For further information, we refer the interested reader to a recent comprehensive review on this topic (Chang et al., 2020).

3D culturing of tumor cells from biopsies or tumor resections has been used during the past decades to study brain tumor biology (Klein et al., 2020). In contrast to cultures of stem cell-derived or primary neural cells, tumor cells can be cultured in chemically defined conditions, which facilitates mechanistic analyses (Hubert et al., 2016). Furthermore, they can be studied in vivo after implantation into the brain of immunodeficient mice to generate patient-derived orthotopic xenografts (Huszthy et al., 2012). However, these models are less suitable to study oncogenesis, and their use for pharmacological applications is limited by the absence of interaction between healthy and tumor tissue (in vitro models) or the lack of scalability (patient-derived orthotopic xenografts). To overcome these issues, two recent studies have used genomeediting to introduce oncogenic mutations into human cerebral organoids. CRISPR/Cas-mediated introduction of oncogenic HRas ${ }^{\mathrm{G} 12 \mathrm{~V}}$ into a subset of cells in undirected cerebral organoids after 4 months in culture resulted in the transfected cells displaying invasive phenotypes within the organoid and a gene expression profile consistent with mesenchymal subtype human glioblastoma (Ogawa et al., 2018). Furthermore, stereotactic injection of organoid-derived tumor cells into the brain of immunodeficient mice allows the study of disease pathology and invasive growth in vivo. In a similar approach, brain tumorigenesis was recapitulated by introducing different oncogenic mutations in undirected cerebral organoids at the end of the neural induction phase by transposon- and CRISPR/Cas9mediated mutagenesis (Bian et al., 2018). This strategy allowed to define combinations of mutations that result in glioblastoma-like and primitive neuroectodermal tumor-like neoplasms.

During the Zika virus (ZIKV) epidemic in the Americas, a dramatic increase in newborns with microcephaly as well as other neurologic impairments was observed (Teixeira et al., 2016). An initial study showed that ZIKV could infect human iPSC-derived neural progenitors in $2 \mathrm{D}$ culture and cause dysregulation of cell cycle, attenuation of proliferation, and cell death (Tang et al., 2016). In agreement with these findings, infection of undirected cerebral organoids with ZIKV in 3D resulted in an average growth reduction by $40 \%$ (Garcez et al., 2016). Infection in directed forebrain organoids revealed tropism toward more immature $\mathrm{SOX}_{2}^{+}$radial glia cells as compared with $\mathrm{TBR}^{+}{ }^{-}$intermediate neural progenitors and immature neurons (Qian et al., 2017b), a finding that was confirmed in studies of fetal human brain slices (Onorati et al., 2016). This established cerebral organoids as a useful platform for studies of neuroinvasive viruses. With the growing number of reported neurologic symptoms in patients with COVID-19, the number of studies in which SARS$\mathrm{CoV}-2$ is used to infect cerebral organoids is steadily increasing (Jacob et al., 2020). However, as compared with ZIKV, findings regarding infectivity and viral tropism are less clear. A general limitation in most cerebral organoid models used to study infectious disease is the lack of nonectodermal microglia (i.e., the resident immune cells of the brain parenchyma). Thus, more recent protocols, which either modify undirected organoids to innately develop microglia-like cells (Ormel et al., 2018) or add iPSC-derived microglia-like cells grown in 2D to directed organoids (Abud et al., 2017), are likely to play a prominent role in future modeling of diseases with pronounced immune components.

\section{Heart}

Cardiovascular toxicity is the most common cause of serious adverse drug reactions in clinical drug development and the most frequent reason for drug withdrawal in postmarketing stages (Ferri et al., 2013; Waring et al., 2015). The mechanisms and clinical classifications of cardiotoxicity are diverse, with QT prolongation associated with inhibition of ether-à-go-gorelated gene (ERG) potassium channels being the most common (Mladěnka et al., 2018). As a consequence, in vitro testing of the effect of a drug candidate on ERG potassium currents is mandated before the compound can enter clinical trials. As traditional studies based on intact organs (in vivo) or freshly isolated primary cells (ex vivo) from animals or patients have proven insufficient, there is a need for new preclinical tools that can better predict cardiac safety and efficacy. New opportunities include genetic screening, bioinformatics, artificial intelligence, and experimental assays powered by human iPSC-derived cardiomyocytes (Sinnecker et al., 2014; de Korte et al., 2020).

\section{A. Single Cell-Based Cardiac Assays}

The function of a single, isolated cardiac muscle cell is defined by three major signals. First, the AP is the stereotypical time course of the transmembrane electrochemical potential as selectively permeable channels transiently exchange ions across the membrane. Second, the calcium transient describes the brief elevation of the cytoplasmic calcium ions as the AP triggers a large calcium release by intracellular storages. Finally, cardiomyocytes exert force as the transiently available cytoplasmic calcium facilitates sarcomere shortening, cell deformation, and tension generation (Fig. 7A). 
1. Electrophysiology Assays for Single Cardiomyocytes. The patch clamp is the gold standard for measuring single-cell APs and ionic currents. Manual patch clamping of isolated single cardiomyocytes has facilitated the development of treatments for channelopathies, such as the long QT syndrome, and the technique was used to study the distribution of ion channels in engineered cell pairs (Aratyn-Schaus et al., 2016; van der Voorn et al., 2020). Automated patch-clamp systems trade control over cell environment for the ability to patch hundreds of cells at a time (Danker and Möller, 2014). The most well established application of automated patch clamp in cardiotoxicity assessment is the ERG activity assay, which measures the effect of a compound on the potassium current flowing through ERG channels genetically expressed in easy-to-patch noncardiomyocytes, such as oocytes. Even if these cells do not have an AP, changes to the ERG current are considered proarrhythmic, since mutations to ERG have been linked to arrhythmias in vivo. Unfortunately, this approach is prone to many false positives as proarrhythmic signals in the expression system do not necessarily translate into human cardiac toxicity, wherein tissue- and organ-level compensatory mechanisms exist. To combat this trend, an international public-private partnership known as comprehensive in vitro proarrhythmia assay was tasked with developing computational models to interpret the ERG results in the context of human heart pathophysiology and whose predictions will be validated against suitable in vitro assays (Ridder et al., 2020).

2. Excitation-Contraction Coupling in Single Cardiomyocytes. Calcium transients can be measured optically using fluorescent dyes. Conventional calcium-sensitive fluorophores are used for comparing relative changes in the same cell, whereas ratio-metric dyes, such as Furared, are characterized by two distinct calcium-sensitive signals, wherein their ratio creates a signal proportional to the absolute level of calcium in the cell (Aratyn-Schaus et al., 2016).

Single-cell contractility can be assessed with miniaturized force transducers, but such analyses are lowthroughput and invasive (Wang and Lin, 2007). Instead, optical analyses of cellular shortening offer minimal interference and high throughput. For instance, the raw displacement and beat rate of cells may be obtained using open-source software, such as MUSCLEMOTION (Sala et al., 2018), which is also applicable for tissuelevel preparations (Ribeiro et al., 2017). Alternatively, cell-generated forces can be quantified by tracking cellinduced deformation of soft substrates of known material properties (Wang and Lin, 2007).

In aggregate, single-cell methods are powerful, scalable, and reproducible. Yet, isolated cells lack the important physiologic signals in a native tissue, which is electrically and mechanically coupled in aligned arrangements (Kojima et al., 2006). In turn, these signals have further proven critical for maturating human iPSC-derived cardiomyocytes (hiPSCCMs) (Ronaldson-Bouchard et al., 2018).

\section{B. Classic Monolayer Cultures}

Cardiac cells seeded on planar culturing substrates at a confluent density can form monolayer tissues. This method constitutes the easiest way to establish cell-to-cell contacts for the study of how AP, calcium transients, and tension propagate through electromechanically coupled cells.

Cardiac monolayers are commonly used in microelectrode arrays (MEAs), instrumented cell culture platforms with microscale surface-embedded electrodes to record electric field potential changes with good spatial resolution. The recording is based on total ion channel activity and can hence diminish the risk of false positives commonly detected with ERG channel assays (Meyer et al., 2004). Moreover, recordings from hiPSC-CMs on MEAs correlated well with ex vivo measurements, suggesting MEAs are a highly reliable platform for drug screening purposes in 2D. The collected data contain beat rate, beat interval, spike amplitude, and field potential duration, allowing a comprehensive drug response analysis (Clements and Thomas, 2014; Harris, 2015). MEA systems can also be integrated with impedance-based readouts to allow for drug screening, including both excitation and contraction parameters (Xi et al., 2011).

Tissue electrophysiology can also be observed using voltage- or calcium-sensitive reporter dyes. Combining these sensors with high-speed imaging systems, AP propagation velocity and patterns can be quantified (Park et al., 2019). Moreover, multiwell imaging systems, such as FLIPR, allow for simultaneous recording of electrical and calcium activities, are compatible with high-throughput screenings, and have been successfully used to identify concentrationdependent effects of ERG inhibitors and calcium channel blockers (Lee et al., 2012; Sirenko et al., 2013; Bedut et al., 2016). Calcium sensors can alternatively be genetically encoded in human iPSCderived lines, which circumvents issues related to limited indicator lifetime and reduces intercellular variability (Shinnawi et al., 2015; Huebsch et al., 2016). Recently, AP, cytosolic calcium transients, and contraction cycles of hiPSC-CMs in response to major drug classes have been simultaneously measured using a high-speed microscopy system (van Meer et al., 2019).

In addition to electrophysiology and calcium fluxes, and similarly to single cells, the contractile movements of monolayer cultures can be imaged to assess beating frequency as an indicator for drug response (Sala et al., 2018). Alternatively, instrumented culture plates containing interdigitated electrodes for impedance measurements 
A
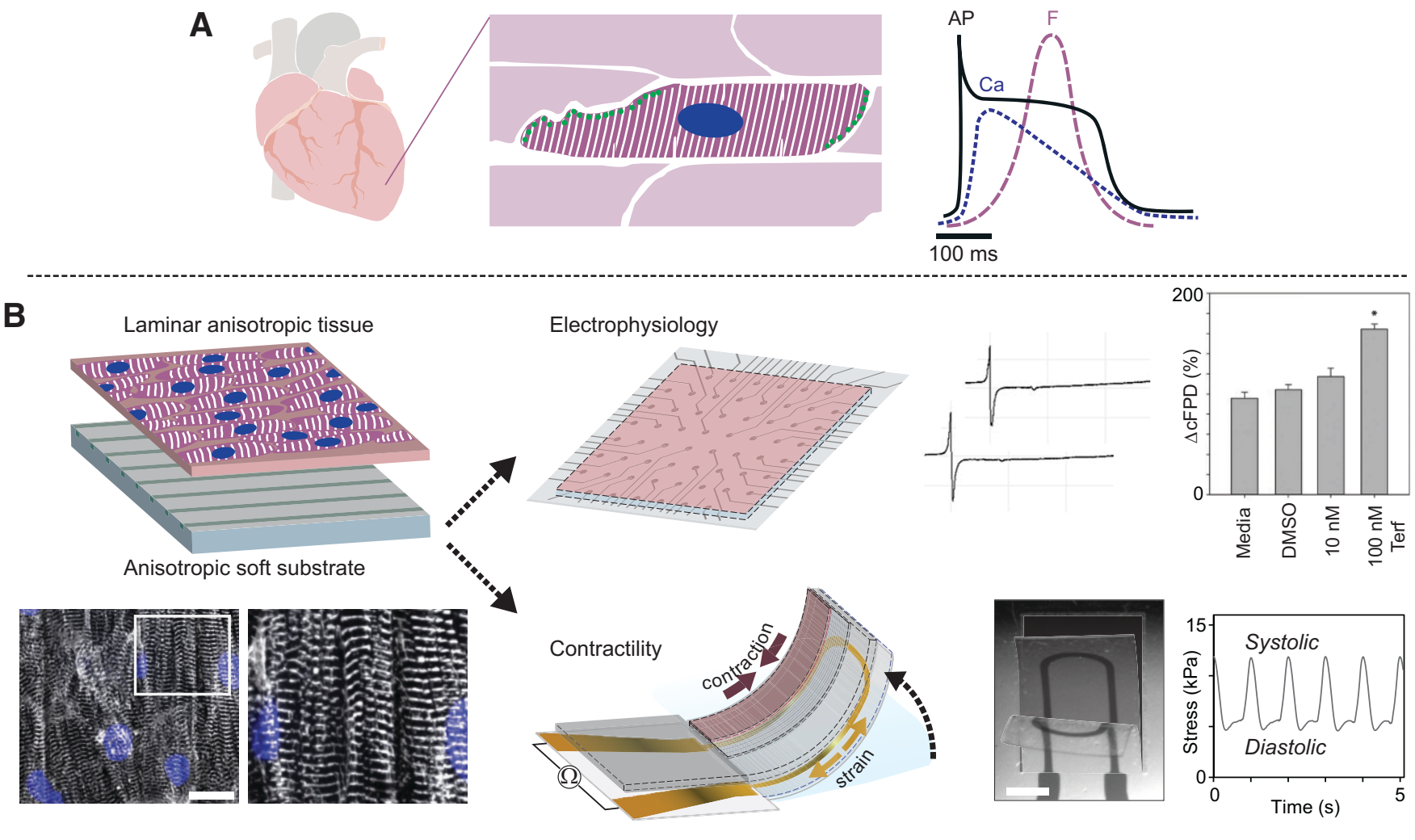

C

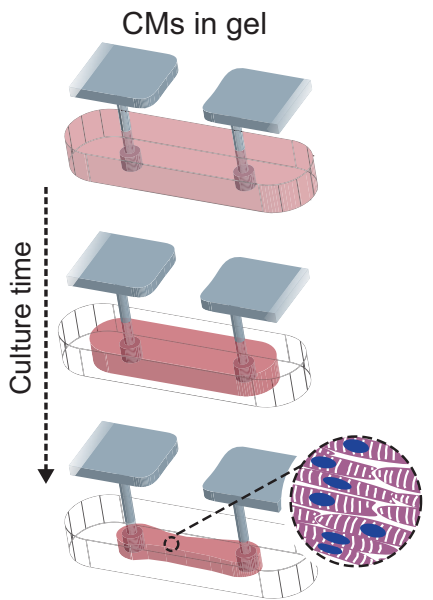

Compact EHT
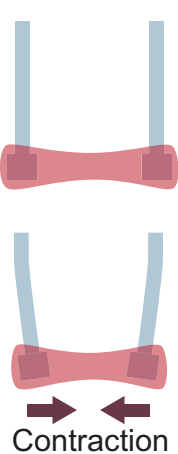

Contraction
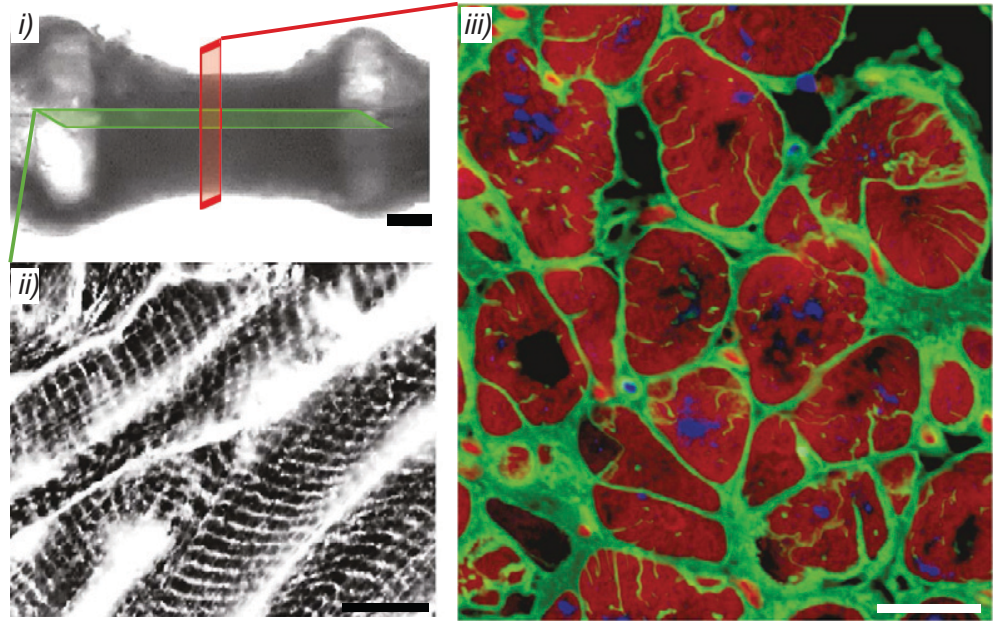

Fig. 7. Organotypic platforms to mimic cardiac function and toxicity. (A) Schematic depiction of the most important endpoints in the evaluation of cardiomyocyte function. The arrival of an AP triggers intracellular calcium transients $(\mathrm{Ca})$, which in turn entail contraction and the generation of contractile force (F). (B) Workflow showing the key principles of engineering laminar tissues and their integration within MEAs for electrophysiological studies or their formulation into contractility assays with embedded soft force gauges. Inlets on the right shows effect of terfinadine (Terf) on rate-corrected field potential duration (DcFPD; top) and an example optical micrograph of a deflecting cantilever with the corresponding electrical readout (bottom). Scale bar $=1 \mathrm{~mm}$. (C) Key principles of the 3D EHT technology. Cardiomyocytes (CMs) encapsulated in a hydrogel are stretched and subjected to electrical stimulation, resulting in tissue compaction and maturation. Insert displays cross-section of a highly mature iPSC-derived EHT with wheat germ agglutinin in green and cardiac troponin $\mathrm{T}$ in red. Scale bars $=500 \mu \mathrm{m}(i)$ and $10 \mu \mathrm{m}$ (ii and iii). Figure modified with permission from (Kujala et al., 2016; Lind et al., 2017b; Ronaldson-Bouchard et al., 2018).

allow for real time noninvasive measurements of contractile cell deformations. These assays are available commercially in multiwell formats and have been applied in hiPSC-CMs drug-dose studies (Nguemo et al., 2012; Scott et al., 2014). Moreover, monolayer cultures were established on circular, thin, flexible silicone membranes to prevent the negative effects of rigid substrates, including hindrance of cell shortening and eventual cell delamination. In these platforms, cardiomyocyte contraction induces deflection of the silicone membrane, which can be tracked to quantify beat rate and contractile force (Linder et al., 2010).

In summary, classic monolayer cultures remain useful in drug screening, as assays are established in 
standardized culture conditions for high-throughput formats coupled to automated analysis. However, conventional planar culture leads to cells with random orientation, whereas the native cardiac muscle is comprised of aligned, end-to-end-coupled, elongated cells.

\section{Tissue Level Models of the Myocardium}

To overcome the limitations of single-cell assays, various tissue-level cardiac models have emerged. These models can be scaffold-free or based on hydrogels or elastomers to mechanically support the tissue constructs. To improve preclinical safety and efficacy assessments, the priority of such complex cardiac models is currently on biologic function, physiology, and phenotypic maturity rather than high-throughput compatibility. Most importantly, there is a need for microphysiological cardiac models to emulate the structural, functional, and electrophysiological similarity to human myocardium as well as to mimic physiologic stretch and fluid dynamics (Pointon et al., 2021). Furthermore, platforms should be compatible with the simultaneous monitoring of multiple functional, morphologic, and pharmacokinetic endpoints.

1. Cardiac Cell Aggregates as Scaffold-Free 3D Models. Spheroid cultures have been developed for the study of 3D cardiomyocyte constructs, which are methodologically similar to spheroids of other tissues. One of the early examples of cardiomyocyte spheroid formation has been performed in 96-well ULA plates in static culture (Koike et al., 2005). The method relies on the spontaneous differentiation of embryonic stem cells in the spherical embryoid body toward different progenitors, including cardiac. Improved methods to aggregate more homogeneous cardiomyocyte spheroids include centrifugation (Burridge et al., 2007) and the use of bioreactors (Lü et al., 2008). A versatile and popular alternative is the hanging drop method, wherein aggregation and cardiomyocyte differentiation of a predefined number of cells occurs in droplets of specified size (Chen et al., 2011). The beat rate of the resulting spheroids can be assayed in response to drug applications either optically (Zuppinger, 2017) or using microelectrodes (Schmid et al., 2016). Embryoid bodies can also be combined with electrical and mechanical stimulation to synchronize and promote iPSC-cardiomyocyte differentiation (Richards et al., 2016; LaBarge et al., 2019).

However, both $2 \mathrm{D}$ monolayers and cardiac spheroids fail to recapitulate the structural architecture of native cardiac cells and tissues. To overcome this problem 3D cardiomyocyte aggregates can be cultured in elongated microfluidic channels (Mathur et al., 2015; Marsano et al., 2016). In this system, the tissues remain viable for many weeks, and their response to drugs is more consistent with ex vivo data compared with single-cell studies. Importantly, such systems can be parallelized to increase throughput (Schneider et al., 2019). Despite these advantages, cardiomyocytes in scaffold-free culture have yet to show similarly elongated morphology, aligned and organized intracellular structure, and directionality in action potential propagation, as found in the scaffolded alternatives.

2. Scaffold-Based Cardiac Tissue Models. Scaffoldbased approaches have been established for replicating the structure and function of the human myocardium. The most prominent strategies include the formation of monolayered or multilayered laminar tissues on deformable substrates or 3D strips derived from deformable scaffolds and external anchoring pillars.

Laminar cardiac tissue models are organized into aligned, anisotropic architectures that display a native-like organization of the contractile cytoskeleton, especially in the alignment and organization of sarcomeres (Fig. 7B). Similarly, the expression and longitudinal localization of gap junctions in laminar tissue enable physiologic AP propagation and can therefore provide more relevant tissues for drug screening and disease modeling. Substrate cues or patterns that induce laminar tissue formation include microcontact printed ECM proteins, micromolded hydrogels, 3Dprinted soft substrates, and nanofiber coatings (Grosberg et al., 2011; Wang et al., 2014; Li et al., 2016a; Lind et al., 2017a; Ahn et al., 2018).

Like conventional monolayer cultures, laminar tissues are thin enough to be compatible with conventional epifluorescent or confocal imaging techniques. Laminar tissue can also be readily integrated with noninvasive electrophysiological assays, such as MEAs (Kujala et al., 2016; Li et al., 2016a; Alassaf et al., 2019). This can serve to overcome the common drawbacks of MEA studies, which include unphysiologic tissue architectures, limited culture time, and tissue delamination.

By formulating the tissue-guiding substrates into deflectable cantilevers, the laminar tissues can be purposed into simple tissue-scale contractility assays. As the tissue contracts, the cantilever deflects proportionally to the contractile force (Grosberg et al., 2011; Wang et al., 2014). Initially cantilevers were made of silicones, but softer formulations derived from gelatin hydrogels were later introduced to extend tissue culture time. More recently, soft, multilayer cantilevers embedding flexible strain gauges were introduced, thus replacing optical tracking of cantilever motions with noninvasive electrical readouts (Lind et al., 2017a,b; Kim et al., 2020a). This enables scalable drug dose-response studies in user-friendly multiwell formats as well as noninvasive monitoring of the long-term maturation of laminar human iPSC-derived cardiomyocyte tissues (Lind et al., 2017a,b).

Engineered tissue strips, also referred to as engineered heart tissues (EHTs), are the most established 3D cardiac tissue models (Fig. 7C). Although 
multiple variations have been reported, they are based on the same key principles. First, a mold containing a set of micropillars or beams that serve as anchoring points for the EHT is fabricated. Then, cardiomyocytes are seeded within a deformable hydrogel matrix, which over time is compacted around the anchoring points by cellular contractions and enzymatic activity, generating an assayable, dense 3D tissue.

The most common formulations rely on collagen combined with laminin or fibronectin introduced directly or by addition of matrigel, which may further be modulated by addition of fibrin or by regulating cell-induced matrix turnover (Ronaldson-Bouchard et al., 2018). Dependent on the mold design, EHT can be formulated as linear or circular strips at a range of sizes, and the anchoring pillars provide either isometric or auxotonic load dependent on their stiffness. To enable miniaturization, molds are often fabricated through PDMSbased soft lithography (Hansen et al., 2010; Boudou et al., 2012; Ronaldson-Bouchard et al., 2018) but have also been made from stereolithographically 3D-printed poly(ethylene glycol) diacrylate hydrogels (Ma et al., 2019) or in multitool approaches that avoid PDMS (Zhao et al., 2019).

EHTs are particularly relevant for contractility studies, which can be performed with force transducers (in the case of large tissue constructs) or by optically tracking the movement of the anchoring pillars. The contractility readouts are often combined with optical electrophysiological assays, such as voltage- and calcium-sensitive sensors based on dyes and proteins.

Importantly, several studies have demonstrated improved maturation of stem cell-derived cardiomyocytes when integrated into EHTs, leading to more adult, tissue-level drug responses. Improved tissue maturation is in part a result of the passive mechanical stretch provided by the anchoring points (Abilez et al., 2018) and can be accelerated drastically by electrically induced contractile training (Hirt et al., 2014) or active mechanical loading (Liaw and Zimmermann, 2016). Moreover, multicellular constructs can be applied to generate EHTs with coupled atrial/ventricular phenotypes (Zhao et al., 2019).

3D EHTs have provided the most adult-like models of the cardiac muscle derived from human iPSC-CMs. Miniaturized assays that combine EHTs with automated video analysis of multiwell plates are by now commercially available and constitute promising systems for medium- to high-throughput drug screening and disease modeling purposes.

\section{Applications of Organotypic Models of the Heart}

1. Cardiotoxicity. As illustrated above, patch clamping, MEAs, and various imaging methods have long been used to monitor cardiac function and constitute an important pillar of high-throughput cardiotoxicity screening. The potential to increase predictivity by incorporating human genetics with iPSC-CMs is further enhanced when engineered culture conditions can provide native-like, mature tissue-level performance. As the use of these engineered culture platforms is becoming more widespread, their predictive power was benchmarked against a gold standard provided by the hERG assay and in vivo data. Commonly used compounds include isoproterenol and epinephrine that increase (positive inotropy) or verapamil and nifedipine that decrease (negative inotropy) contractile force, respectively.

As a simplistic and easy-to-scale 3D platform, cardiac spheroids in multiwell plates for calcium imaging are currently the preferred platform for cardiotoxicity screening (Sirenko et al., 2017). For instance, spheroids formed of cocultured hiPSC-CMs and fibroblasts (Verheijen et al., 2018) or endothelial cells (Polonchuk et al., 2017) in physiologic ratios have successfully identified the dose-dependent toxicity of doxorubicin, a cancer therapeutic with known cardiac side effects, which complemented earlier findings derived from traditional monolayer cultures (Burridge et al., 2016). Furthermore, spheroid testing of 29 and 13 compounds with known inotroptic and noninotroptic effects in vivo in 384-well format under field stimulation in an automated calcium imaging system resulted in $80 \%$ sensitivity and $91 \%$ specificity (Pointon et al., 2017).

Laminar-tissue cantilever assays provide force readouts and allow long-term culturing, rendering them suitable for nonacute in vitro studies (Grosberg et al., 2011; Ahn et al., 2018). Instrumented cantilevers with embedded sensors in multiwell formats have allowed noninvasive monitoring of the contractile maturation of hiPSC-CM tissues over a 4-week period and 12 doseresponse studies of positive and negative inotropic compounds (Lind et al., 2017a,b).

Although high-throughput drug screening using EHTs was initially challenging because of their size, several approaches achieved suitably small tissues (Hansen et al., 2010; Boudou et al., 2012), and EHT constructs have by now been established in 96-well format (Mills et al., 2017; Thavandiran et al., 2020). Known compounds such as quinidine, chromanol, erythromycin, and doxorubicin have been tested on EHTs in dose-dependent manner (Hansen et al., 2010). In a recent intersectoral, multicenter drug screening study EHTs achieved 93\% accuracy for drug-induced cardiac response (Saleem et al., 2020). Responses aligned most closely to the free therapeutic plasma concentration, and contraction amplitude was found to be a good predictor of negative and positive inotropes in the 3D EHT culture, underlining the value of iPSC-CM-based EHTs for drug safety evaluation. 
2. Cardiac Disease Models for Drug Development. Although confidence in engineered cell culture platforms increases with the addition of more and more compounds into validation libraries, these platforms are also being rapidly adopted for discovery and efficacy studies, especially in those cases wherein an animal model of the disease of interest is not available. Current state-of-the-art engineered cardiac tissue models were initially built from primary rodent cell sources and were validated by exposure to several compounds with known effects (Grosberg et al., 2011). However, there is a lack of studies validating engineered cardiac tissue models based on stem cells, in particular with cells originating from larger animals applied in preclinical cardiac research, such as dog. The importance of cell source is underlined by recent research showing that engineered tissues based on primary rodent and stem cell-derived human tissues display markedly different response to potential emerging exosome therapies against myocardial infarct (Yadid et al., 2020).

Long QT syndrome is an inherited arrhythmogenic syndrome characterized by aberrant ion channel function leading to sudden heart failure. Using patientderived iPSC from diseased individuals, in vivo phenotypes can be recapitulated in vitro, as validated by electrophysiological studies that reveal action potential prolongation through patch-clamp and MEA assays (Itzhaki et al., 2011; Malan et al., 2016). The diseased phenotypes could be pharmacologically rescued in vitro as proof of successful tissue modeling. Congenital long QT syndrome can furthermore be modeled in 2D using fully automated 384-well assays based on voltage sensitive dyes. This may enable testing of patient cells at early stages of drug development and predict clinical outcomes of patient-specific differences (McKeithan et al., 2017).

Patient-specific iPSC BRAF mutant cells (Cashman et al., 2016) and genome-edited MYH7 mutant lines (Mosqueira et al., 2018) can be used to model dysfunction of the left ventricle. EHT constructs generated from these cells recapitulate in vivo tissue morphology with altered contractility and typical electrophysiology with accelerated twitch dynamics as well as altered signaling and metabolism. Likewise, dilated cardiomyopathy can be modeled in vitro using genetically edited iPSC-derived cardiomyocytes with mutations in TNNT2 (Sun et al., 2012) or TTN (Hinson et al., 2015). Cells from TNNT2 variant carriers showed reduced beating rates, compromised contraction, and abnormal sarcomeric $\alpha$-actinin distribution, which could be partially rescued by treatment with blockers of $\beta$-adrenergic signaling, whereas TTN mutations manifested in aberrant sarcomeric bands, sarcomere insufficiency, and impaired response to adrenergic and mechanical stress in miniaturized EHTs.

Engineered cell culture platforms also facilitated the study of congenital arrhythmic disease, such as catecholaminergic polymorphic tachycardia (Goldfracht et al., 2019). A recent study with laminar 2D tissues using optogenetics and optical mapping revealed calcium wave re-entry as the substrate for the life-threatening arrhythmias (Park et al., 2019). By using genome editing the authors traced the phenotype to a single catecholamine-driven phosphorylation event of the ryanodine receptor, which helps in the development of treatment strategy.

Barth syndrome is a monogenic mitochondrial myopathy resulting in global ATP depletion, disrupted sarcomeric architecture, and decreased contractile stress generation. The in vitro model exhibited abnormalities in sarcomere assembly and myocardial contraction typical of the disease (Wang et al., 2014). Interestingly, using genetic and pharmacological rescue experiments, the model revealed that these aberrations were not linked to the lack of ATP per se as previously believed but rather with the loss of mitochondrial reactive oxygen species buffering capacity. Familial hypertrophic cardiomyopathy is a hereditary cardiac disorder characterized by cardiac hypertrophy associated with mutations in structural genes, such as MYH7 (Lan et al., 2013), MYBPC3, and ACTN2 (Prondzynski et al., 2019). EHT cultures of cardiomyocytes generated from patient-derived iPSCs replicate myocyte hypertrophy and myofibrillar disarray, leading to hypercontractility, impaired relaxation, and electrophysiological abnormalities with higher calcium sensitivity and prolonged action potential duration (Prondzynski et al., 2019).

Although patient-specific and genome-edited iPSCs enable the in vitro investigation of genetic diseases, most cardiovascular disease cases are associated with environmental conditions, such as arterial blockages and myocardial infarction. One of the early examples to model such factors in EHTs focused on effects of increased afterload on cardiac hypertrophy (Hirt et al., 2012). After 2 weeks, the soft PDMS posts were reinforced with metal braces leading to EHTs enlargement by $28.4 \%$ together with metabolic signs of hypertrophy and reduced contractile force after release. Cardiac fibrosis is another nonhereditary condition leading to reduced contractility and, in severe cases, heart failure. TGF $\beta$-activated fibroblasts were mixed with cardiomyocytes, and the resulting EHTs recapitulated the distinctive features of cardiac fibrosis, including excessive collagen deposition, increased tissue stiffness, and passive tension (Mastikhina et al., 2020). Notably, treatment with the antifibrotic drug pirfenidone reduced tissue stiffness and reversed fibrosis-associated transcriptomic alterations, suggesting the utility of this platform for drug discovery. To recapitulate diseases that manifest in specific areas of the heart, a recent EHT platform was populated with iPSC-derievd cardiomyocytes differentiated to recapitulate the characteristics of atrial and 
ventricular cardiomyocytes. The platform enabled the characterization of normal electrophysiological and contractile parameters as well as the pathophysiological and pharmacological characteristics of the atrioventricular muscle (Zhao et al., 2019).

Ischemia-reperfusion injury (IRI) was also mimicked in vitro in EHTs by first culturing cells for 6 hours in low-medium volumes in a hypoxic chamber perfused by anoxic gas to emulate the nutrient deprivation, hyperkalemia, high lactate concentration, and low extracellular $\mathrm{pH}$ observed in cardiac ischemia (Chen and Vunjak-Novakovic, 2019). Subsequently, ambient oxygen concentrations were restored, and fresh medium was added to replenish nutrients and re-establish $\mathrm{pH}$. IRI resulted in elevated ROS, increased lactate dehydrogenase release, and caspase activity as well as decreased contractility, which could be ameliorated using ischemic preconditioning in alignment with in vivo data. In addition, engineered human laminar tissues with embedded contractility sensors were used to monitor the reduction in contractility during IRI in real time (Yadid et al., 2020). Furthermore, the authors showed the therapeutic potential of human extracellular vesicles derived from endothelial cells and could trace the protective effects to protein modules involved in AMPK signaling, redox homeostasis, and unfolded protein response. Combined, these results suggest that the presented models might constitute interesting platforms for the development and translation of novel therapeutic approaches for IRI.

The number of examples using hiPSC-CMs on engisystem for both genetic and nongenetic heart pathologies. Faithful disease modeling of these conditions carries a high translational potential by pinpointing potential targets, screening for novel drug candidates, and facilitating the identification of patient-tailored solutions, particularly for rare conditions.

\section{Skeletal Muscle}

Skeletal muscle accounts for $30 \%-50 \%$ of human body weight and fulfills crucial functions related to movement, posture, and metabolic homeostasis. Skeletal muscle is characterized by a highly organized hierarchical architecture in which individual neered in vitro models emphasizes the versatility of the

myofibers are grouped into myofiber bundles or fascicles surrounded by an ECM layer, the perimysium. These myofibers are moreover associated with satellite cells, adult stem cells that are tethered to the basal lamina and contribute to muscle regeneration upon injury (Mukund and Subramaniam, 2020).

Pharmacologically, skeletal muscle constitutes a tissue of interest, primarily for its role in glycemic control and metabolic disease. Furthermore, evaluation of muscle toxicity can be of relevance in drug development. Importantly, common preclinical animal models do not accurately mimic human metabolic disease or drug-induced dystrophies, resulting in substantial efforts to develop more accurate preclinical models of human skeletal muscle. This need was emphasized by the increased mortality primarily allotted to fatal rhabdomyolysis and associated with cerivastatin, a drug that was well tolerated in multiple preclinical species, including mice, rats, rabbits, dogs, minipigs, and monkeys (von Keutz and Schlüter, 1998; Thompson et al., 2006). In the last years, impressive progress has been made toward the bioengineering of in vitro skeletal muscle constructs from human primary myoblasts or pluripotent stem cells (Jalal et al., 2021).

\section{A. Primary Myoblast-Derived 3D Culture}

Most 3D primary muscle culture models are based on the use of primary CD56-positive myogenic satellite cells obtained from needle biopsies for differentiation into mature myofibers (Table 6). Differentiation protocols are heterogeneous regarding molecules used for differentiation, time frames, and culturing methods, such as anchoring cells to a fibrin-based 3D environment, hydrogel molding (Hinds et al., 2011; Juhas and Bursac, 2014), or custom-made microfluidic devices (Zahavi et al., 2015). Most methods rely on the embedding of myoblasts into hydrogel and the subsequent compaction and alignment of myofibers around fixed anchorpoints (Fig. 8, A-C). Differentiated myotubes exhibited main characteristics of mature myocytes, including alignment of multinucleated myofibers in a laminin-rich matrix, expression of muscle-specific proteins (myosin heavy chain, sarcomeric $\alpha$-actinin and muscle creatine kinase), and physiologically relevant $\mathrm{Ca} 2+$ handling and force generation upon electrical stimulation (Chiron et al., 2012; Madden et al., 2015)

TABLE 6

Table with strengths/weaknesses of skeletal muscle models

\begin{tabular}{lccc}
\hline & Pluripotent Stem Cells & Primary Myoblasts & Mature Myofiber Cultures \\
\hline Maturation method & Transgene- or small molecule-based & Transgene- or small molecule-based & Not applicable \\
Phenotype & + & ++ & +++ \\
Length of differentiation protocol & + & ++ & Not applicable \\
Stability in culture & +++ & ++ & + \\
Accessibility & +++ & ++ & + \\
Homogeneity of culture & + & ++ & ++ \\
Functionality & + & ++ & +++ \\
Current use in drug testing & +++ & ++ & Not tested yet \\
\hline
\end{tabular}



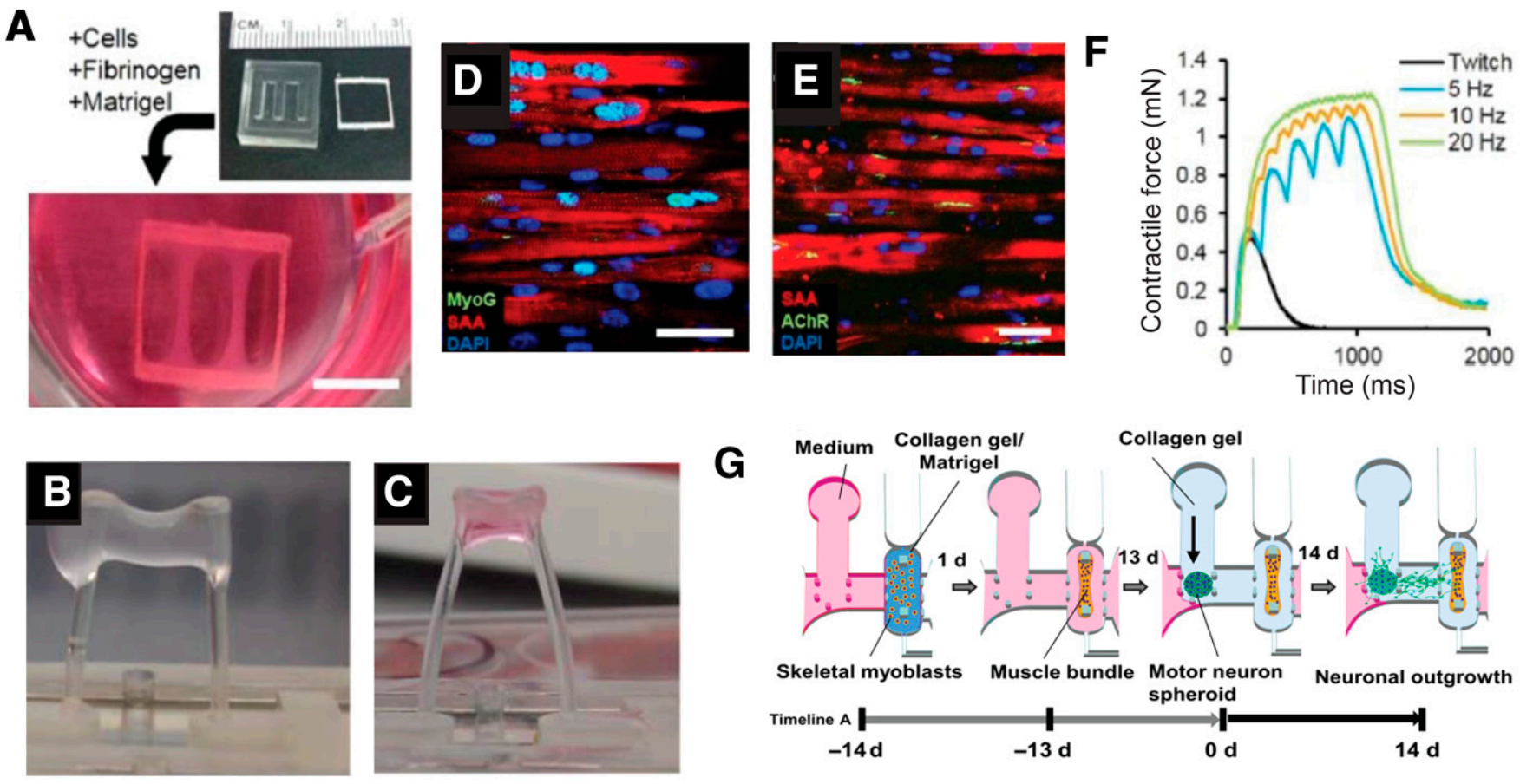

H

Control motor unit

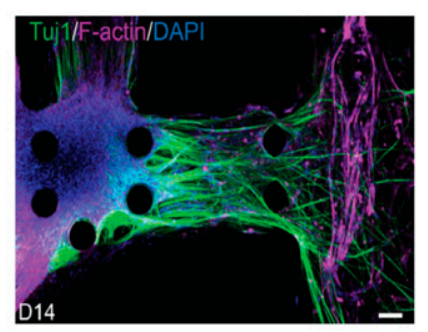

ALS patient-derived motor unit

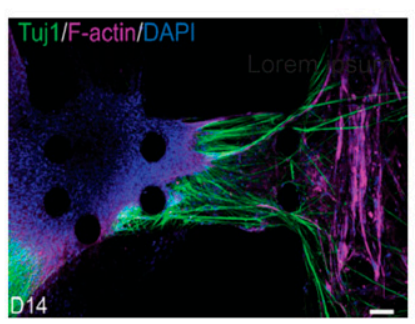

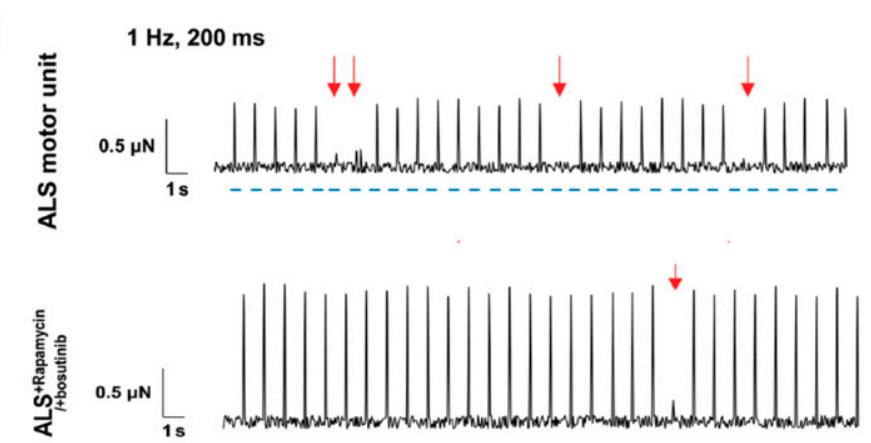

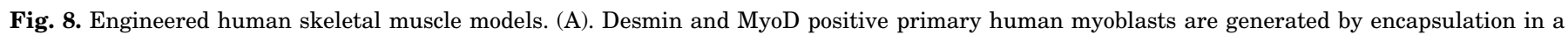

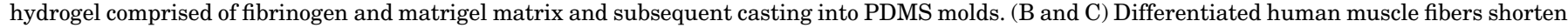

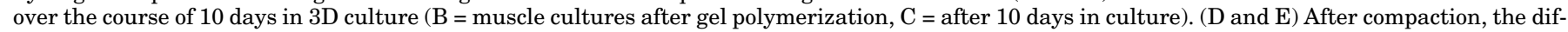

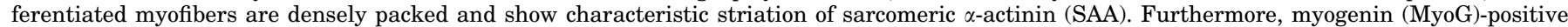

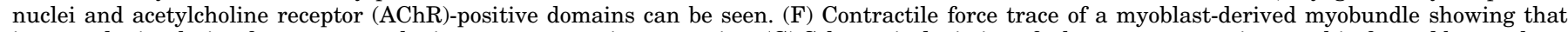

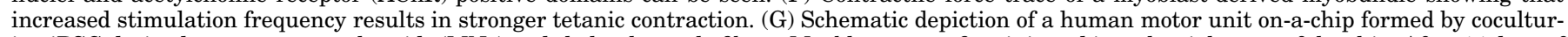

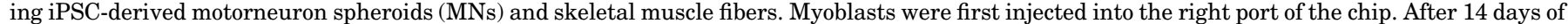

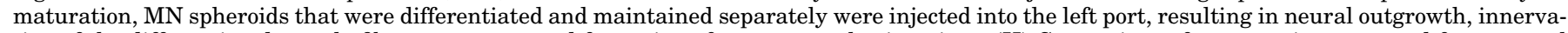

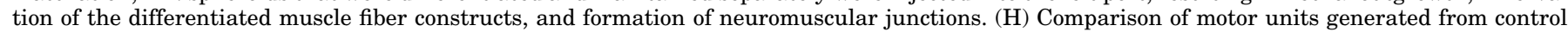

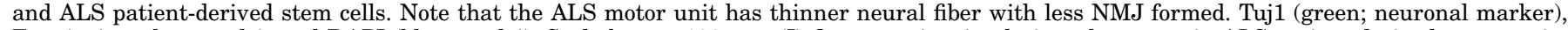

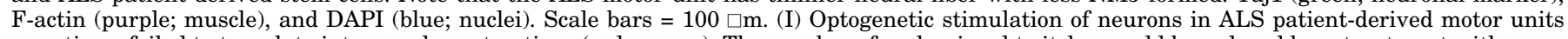

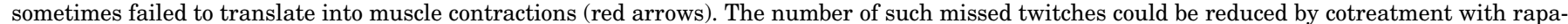

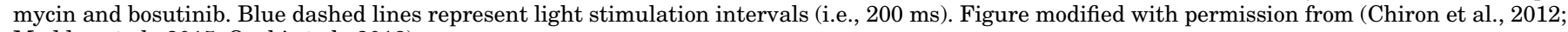
Madden et al., 2015; Osaki et al., 2018).

(Fig. 8, D-F). Furthermore, they remain functional in culture for at least 4 weeks. Their physiologic relevance of the system was moreover strengthened by the expression of responsive acetylcholine and $\beta$-adrenergic neuromuscular junction (NMJ) receptors and the presence of a small pool of basal lamina-associated paired box gene (PAX) 7-positive satellite cells.

To study the NMJs using isogenic cells, primary human myoblasts were reprogrammed into iPSCs, expanded, and differentiated into both myotubes and motor neurons (Vila et al., 2019). The generated myotubes were multi- nucleated and expressed mature muscle markers, such as $\alpha$-actinin, MYOD, desmin, and myogenin. Such myofibermotor neuron cocultures result in synaptic colocalization and allow for acetylcholine- and glutamic acid-based stimulation of human myocytes (Dixon et al., 2018; Santhanam et al., 2018). Furthermore, the evaluation of known NMJ inhibitors, such as bungarotoxin and curare, resulted in dose-dependent inhibition of muscle responses at relevant concentrations, suggesting the pharmacological relevance of such systems. However, major limitations of primary models are the paucity of biopsy material, limited 
proliferation, and a decline in differentiation ability after prolonged culture (Machida et al., 2004).

\section{B. Pluripotent Stem Cell-Derived Muscle Models}

To overcome these limitations associated with the use of primary cells, iPSCs can be used as substrate for the bioengineering of skeletal muscle models. Both human iPSCs and ESCs can be lineage-committed to myogenic progenitor cells positive for the early myogenic markers PAX3 and MYF5 but negative for the later stage markers MYOD and MYOG using either small molecule-based protocols (Barberi et al., 2007; Chal et al., 2016) or direct reprogramming (Darabi et al., 2012). Reprogramming protocols based on the overexpression of myogenic transcription factors are faster and result in the generation of more homogeneous myogenic progenitor populations.

Upon differentiation in 2D culture, cells became spindle-shaped and fused into spontaneously contracting multinucleated myotubes with few associated PAX7-positive cells, resembling the localization of satellite cells in vivo (Rao et al., 2018). Although cells deteriorated after 2 weeks in $2 \mathrm{D}$ culture, embedding of these myotubes in fibrin-based hydrogels resulted in densely packed, aligned, cross-striated myotube bundles surrounded by a network of laminin- and collagen I-rich ECM. These bundles were responsive to chemical or electrical stimulation, and, upon transplantation into immunodeficient mice, they engrafted, became vascularized, and remained functional. However, the specific tetanic force generated by those bundles $\left(\sim 3 \mathrm{mN} / \mathrm{mm}^{2}\right)$ remained considerably lower than in age-matched adult human bundles $\left(\sim 7-12 \mathrm{mN} / \mathrm{mm}^{2}\right)$ and even fetal muscle $\left(\sim 6 \mathrm{mN} / \mathrm{mm}^{2}\right)$.

3D bundle cultures of differentiated myotubes under unidirectional tension were moreover extended into multilineage models by the addition of endothelial cells and pericytes (Maffioletti et al., 2018). Although no clear benefits on muscle function or molecular phenotypes were demonstrated, the authors showed that addition of these isogenic cells resulted in the formation of vessel-like structures, increasing histologic complexity. The stem cell-derived in vitro models of human skeletal muscle discussed above allow for qualitative and quantitative measurements of functional endpoints, rendering them promising tools for pharmacological testing.

\section{Applications of 3D Human Skeletal Muscle Models}

Compared with other 3D models, such as of liver, intestine, and heart, the use of 3D skeletal muscle models is less widespread. Pharmacological and toxicological applications of human skeletal muscle models are particularly in preclinical myotoxocity screening and for the development of potential therapies against muscle diseases. Further common applications in regenerative medicine are beyond the scope of this work, and readers are referred to excellent recent reviews (Wang et al., 2019a; Kang et al., 2020).

1. Muscle-Specific Toxicity. Myotoxicity of drug candidates is primarily evaluated using cell viability endpoints, which can furthermore be complemented with measurements of contractile force, oxygen consumption rate, calcium ion transient, and fatigue resistance. Studies presented so far have evaluated the myotoxicity of few selected compounds, whereas comprehensive screens have not yet been presented. For instance, cerivastatin and lovastatin caused progressive weakness and lipid accumulation in 3D myoblast-derived microphysiological myobundle cultures (Juhas and Bursac, 2014; Madden et al., 2015). Notably, reduced force production was observed at therapeutically relevant concentrations of the myotoxic statin cerivastatin ( $5 \mathrm{nM})$, whereas reduced force generation due to lovastatin treatment was only observed at 40-fold higher concentrations (200 nM). Mechanistically, in vitro experiments demonstrated that cerivastatin myotoxicity was mediated by OATP2B1mediated statin uptake, whereas MRP1 overexpression on the sarcolemmal membrane ameliorated statininduced myopathy (Knauer et al., 2010).

Besides statins, 3D muscle bundles successfully recapitulated chloroquine-induced autophagic myopathy as well as hypertrophy and increase in contractile strength upon exposure to the $\beta$-adrenergic agonist clenbuterol, mimicking effects observed in vivo (Madden et al., 2015). Moreover, live imaging of acetylcholine-induced calcium release using myogenic cells engineered to express the calcium reporter GCaMP6 revealed inhibition of calcium release upon treatment with the muscle relaxant tubocurarine. Overall, these results provide proof of principle that microphysiological muscle models can recapitulate the functional responses to pharmacological stimuli of native human muscles, suggesting that such systems might pose promising preclinical screening platforms for myotoxicity testing.

2. Mitochondrial Toxicity. Drug-induced mitochondrial dysfunction constitutes a common off-target toxicity not always identified during early drug development stages that commonly manifests in muscle injury (Dykens and Will, 2007). The difficulty to detect mitochondrial toxicity prior to clinical trials is mainly due to the lack of physiologically relevant cellular oxidative stress indicators in existing screening platforms and the relatively short duration of many preclinical studies. To this end, functional human 3D muscle models constitute an ideal system to evaluate mitochondria toxicity because of their high energy demands. Specifically, primary myoblastderived 3D models allow for measurements of respiration rate using polarographic high-resolution respirometry and muscle fatigue (Davis et al., 2017). Furthermore, by using several mechanistically distinct respiration inhibitors, such as oligomycin (ATP synthase inhibitor), FCCP (electron transport uncoupler), and antimycin A (complex III 
inhibitor), the authors revealed that previously used models for the evaluation of mitochondrial toxicity, such as isolated mitochondria and myotube monolayer cultures, had higher basal respiration rate than bioengineered 3D muscle bundles, possibly due to mitochondrial stress and nontransport-limited oxygen consumption. Importantly, the model was able to recapitulate dose-dependent decrease in respiration and ATP generation upon treatment with the complex I inhibitor rotenone, which propagated into reduced twitch and tetanus force, thus providing proof of principle that $3 \mathrm{D}$ skeletal muscle models can be useful for bioenergetic profiling.

3. Muscle Disease Models. Duschenne muscular dystrophy (DMD) is a severe muscle-wasting disease caused by mutation in the DMD gene. Although few antisense oligonucleotide-based treatments have been approved by EMA and FDA in recent years (eteplirsen, golodirsen, viltolarsen, and casimersen), these drugs are specific for certain mutations. As such, development of novel drugs for DMD therapy remains an area of ongoing research. Drug development is furthermore complicated by the scarcity of models that recapitulate the complexity of the pathology, especially with intricate interplays between skeletal muscle tissues, vascular networks, and fibroblasts responsible for deposition of extracellular matrices, such as collagen I, fibronectin, and $\alpha$-smooth muscle actin.

To study DMD in vitro, 3D myobundle models generated from differentiated immortalized myoblasts were cocultured with endothelial cells to recapitulate CD36+ and CD31+ vascularized endothelial networks (Bersini et al., 2018). The myobundles were also positive for myosin heavy chain marker, suggesting full maturation of the skeletal muscle fibers. Furthermore, introduction of muscle-derived human fibroblasts resulted in the formation of a sheath structure, resembling the connective endomysium found in vivo. Interestingly, incorporation of patient-derived fibroblasts phenocopied the elevated expression of collagen I and fibronectin seen in DMD in vivo, whereas the corresponding $2 \mathrm{D}$ cultures failed to recapitulate the pathophysiology. Notably, however, results are based on cells from a single patient and thus warrant validations.

Amyotropic lateral sclerosis (ALS) is a neuromuscular disease leading to progressive loss of motorneurons, muscular atrophy, and lack of voluntary muscle movements. Ten percent of ALS cases are attributed to SOD1 mutations, whereas the remainder is idiopathic. Two drugs have been approved for symptomatic treatments (riluzole and edaravone); however, both drugs only moderately delay disease progression, and response rates remain low.

To develop high fidelity and robust testing platforms for ALS drug discovery and development, patient-derived iPSCs were used to generate isogenic 3D skeletal muscle fiber models expressing muscle markers (myogenin) and neural spheroids expression markers for motor neurons, such as choline acetyltransferase and TUJ1 (Osaki et al., 2018). The models were cultured in two separate chambers of a microfluidic device, and, after 14 days, neurite elongation from the motor neuron spheroids toward the skeletal muscle fibers resulted in the formation of a mature NMJ (Fig. 8G), which could not be mimicked using conventional 2D cultures (Dimos et al., 2008). Notably, the model showed reduced skeletal muscle innervation with ALS patient-derived motorneuron spheroids (Fig. 8H). To allow for spatiotemporal control of neural activity and muscle contraction, the authors used an elegant optogenetic system that allowed for light-inducible cation influx into neurons. Upon $1-\mathrm{Hz}$ optical stimulation, muscle contraction was observed in muscle fibers connected to either control or ALS-derived motor neurons; however, coupling was $32 \%$ less efficient in ALS neuromuscular junctions. Importantly, treatment with rapamycin and bosutinib reduced dysfunctional signal transmission and increased autophagy, demonstrating the neuroprotective effect of this combination therapy and highlighting the power of the platform for ALS drug development (Fig. 8I).

\section{Fluidic Integration of Organotypic Tissue Models}

$3 \mathrm{D}$ culture systems can support the stable culture of human tissue models comprising one or more cell types, thereby enabling drug testing in physiologic contexts. However, they are limited to one tissue and thus do not allow to study tissue interactions. To overcome this limitation, multiorgan-on-a-chip (MOOC) models have been developed that integrate organotypic culture modules corresponding to different tissues in an interconnected microfluidic circuit. In these chips, the different tissue models are cultured in the same media, thus mandating the compatibility of culture conditions and supplements. As no medium has been presented that can support the culture of all tissues, this need for universal media can limit the choice of possible tissue constellations. Perfusion setups can either be single-pass, in which medium is perfused unidirectionally through the different compartments thus allowing only interaction of tissue A with B but not reciprocally, or recirculating, in which interaction between the tissues is reciprocal (RonaldsonBouchard and Vunjak-Novakovic, 2018). By now, a multitude of different tissue constellations have been presented (Table 7).

Major barriers of MOOC models are that all cocultured models must be compatible with a universal culture medium. Appropriate composition of media and supplements becomes exponentially more challenging with an increasing number of tissue models in the MOOC. Furthermore, as no medium has been presented that can support the long-term culture of 
TABLE 7

Overview of multiorgan-on-a-chip combinations used for pharmacological or toxicological applications

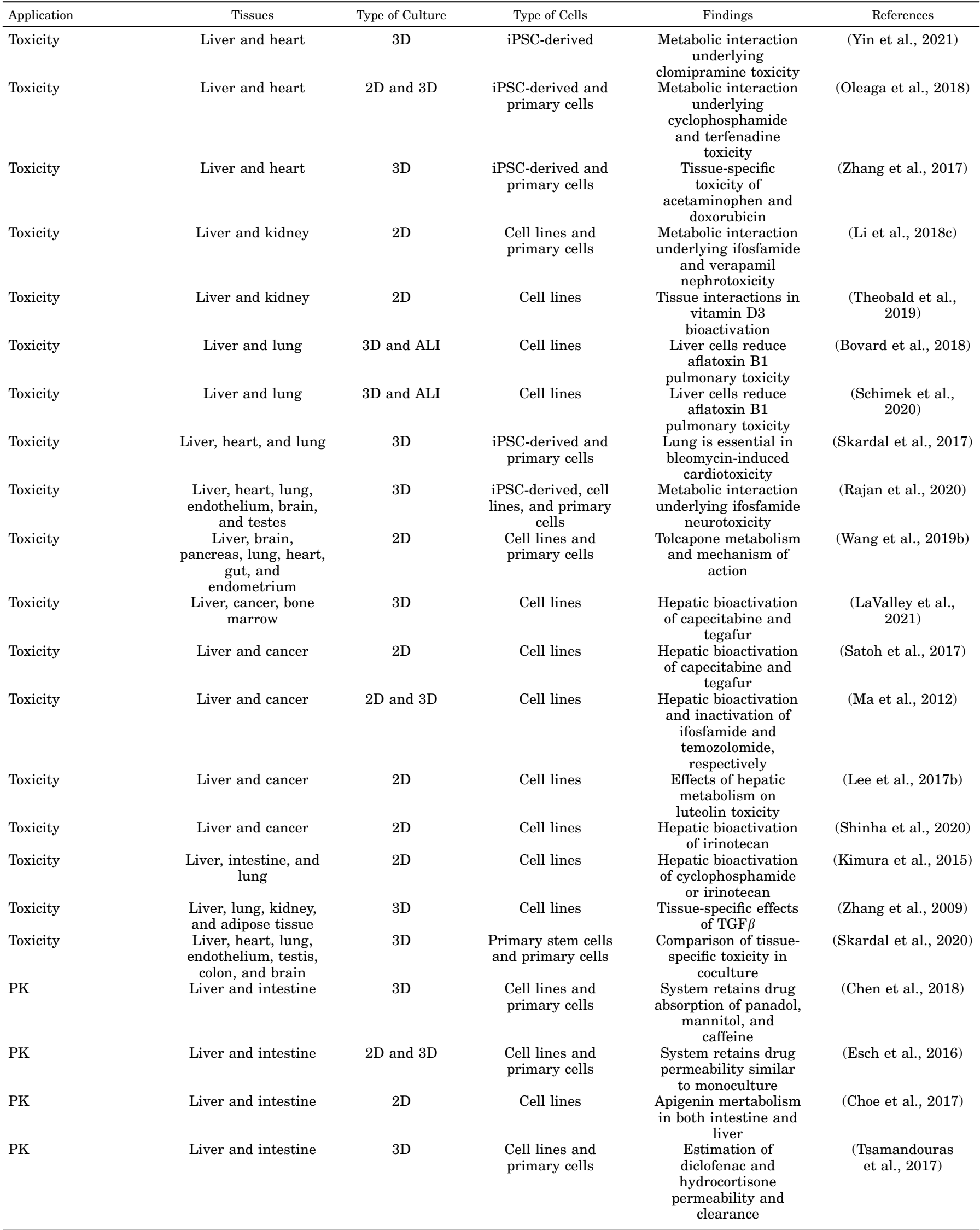




\begin{tabular}{|c|c|c|c|c|c|}
\hline \multirow[b]{2}{*}{ Application } & \multirow[b]{2}{*}{ Tissues } & \multicolumn{2}{|c|}{ TABLE 7-Continued } & \multirow[b]{2}{*}{ Findings } & \multirow[b]{2}{*}{ References } \\
\hline & & Type of Culture & Type of Cells & & \\
\hline PK & Liver and intestine & $2 \mathrm{D}$ & Cell lines & $\begin{array}{l}\text { Absorption of fatty } \\
\text { acid and evaluation of } \\
\text { antisteatotic effect of } \\
\text { metformin and XL- } \\
335\end{array}$ & (Jeon et al., 2021) \\
\hline Gut-brain-axis & Brain and intestine & $2 \mathrm{D}$ & iPSC-derived & $\begin{array}{l}\text { Evaluation of the } \\
\text { impact of the } \\
\text { intestinal microflora } \\
\text { on neurodegeneration }\end{array}$ & $\begin{array}{l}\text { (Raimondi et al., } \\
\text { 2019) }\end{array}$ \\
\hline Gut-brain-axis & $\begin{array}{l}\text { Liver, intestine, and } \\
\text { brain }\end{array}$ & $2 \mathrm{D}$ and $3 \mathrm{D}$ & $\begin{array}{l}\text { Primary stem cells, } \\
\text { cell lines, and } \\
\text { primary cells }\end{array}$ & $\begin{array}{l}\text { Microbiome-derived } \\
\text { short-chain fatty } \\
\text { acids increase the } \\
\text { expression of } \\
\text { pathology-associated } \\
\text { pathways in } \\
\text { neurodegenerative } \\
\text { disease }\end{array}$ & $\begin{array}{c}\text { (Trapecar et al., } \\
\text { 2021) }\end{array}$ \\
\hline Metastasis formation & Brain and lung & $2 \mathrm{D}$ & Cell lines & $\begin{array}{l}\text { NSCLC metastasizing } \\
\text { propensity to brain }\end{array}$ & (Liu et al., 2019) \\
\hline Glucose metabolism & Liver and pancreas & $3 \mathrm{D}$ & $\begin{array}{l}\text { Cell lines and } \\
\text { primary cells }\end{array}$ & $\begin{array}{l}\text { Recapitulation of } \\
\text { glucose metabolism } \\
\text { and homeostasis }\end{array}$ & (Bauer et al., 2017) \\
\hline Glucose metabolism & $\begin{array}{l}\text { Liver, pancreas, and } \\
\text { skeletal muscle }\end{array}$ & $2 \mathrm{D}$ & Cell lines & $\begin{array}{l}\text { Recapitulation of } \\
\text { glucose metabolism } \\
\text { and homeostasis }\end{array}$ & (Lee et al., 2019) \\
\hline
\end{tabular}

ALI, air-liquid interface; NSCLC, non-small cell lung cancer; PK, pharmacokinetics.

all tissues, this need for universal media can limit the choice of possible tissue constellations. Besides the selection of media, the fluidic integration of multiple tissue models is complicated by issues pertaining to the scaling of tissue components. Although early approaches used allometry to guide size and stoichiometry of tissue models in a MOOC, more recent approaches have transitioned to the use of functional scaling, in which quantifiable physiologic parameters, such as metabolism, filtration rate, or glucose consumption, inform model design (Stokes et al., 2015). However, we would like to reiterate previous views that even the most complex MOOCs will and should remain reductionistic in nature (Wikswo et al., 2013). As such, they should be seen as model systems that can, ideally, improve preclinical-to-clinical translation in drug development. In contrast, we believe that hyperbolic terms, such as "human-on-a-chip," mislead about the level of model abstraction and exaggerate the level of complexity that can be recapitulated in MOOCs.

MOOCs involving the liver have been useful for evaluating effects of drug metabolism and hepatic clearance on tissue-specific toxicity. Interesting data demonstrating the utility of this approach have been demonstrated for cocultures of liver and cardiac models. Specifically, hepatic metabolism was shown to cause bioactivation of clomipramine and cyclophosphamide resulting in increased cardiotoxicity, whereas metabolism of terfinadine to its noncardiotoxic metabolite fexofenadine was cardioprotective (Oleaga et al., 2018; Yin et al., 2021). Acetaminophen showed only liver-specific toxicity, whereas only slight cardiac perturbations were detected (Zhang et al., 2017). In contrast, doxorubicin caused both hepatotoxicity and cardiotoxicity, as indicated by increased release of GST $\alpha$ and CK-MB, the bound combination of the CKM and CKB isoenzymes of phosphocreatine kinase; reduced albumin secretion; and strongly decreased beating rate, suggesting that MOOCs can faithfully inform about the primary sites of drug-induced tissue injury.

Metabolic interaction was also demonstrated for liverkidney and liver-lung MOOCs. In liver-kidney models, the hepatic bioactivation of ifosfamide caused significant toxicity in glomerular endothelial cells, whereas metabolic inactivation of verapamil to norverapamil reduced nephrotoxicity ( $\mathrm{Li}$ et al., 2018c). Similarly, coculture of liver and kidney models increased vitamin D3 metabolism and bioactivation on chip (Theobald et al., 2019). For liver-lung chips consisting of a coculture of HepaRG spheroids and human bronchial epithelial cells, the liver compartment was shown to protect the lung cells from aflatoxin B1-mediated toxicity (Bovard et al., 2018; Schimek et al., 2020). Notably, these protective effects were paralleled by substantial toxicity in the liver compartment that likely arises due to the hepatic metabolism of aflatoxin B1 into cytotoxic aflatoxin exo8,9-epoxide.

Interdependent metabolism has furthermore been demonstrated in more complex MOOCs, such as tricultures of liver (PHH + HSC spheroids), heart (iPSC-derived cardiomyocyte spheroids), and lung (3D airway organoids). Specifically, using this setup, the authors reveal indirect bleomycin-induced cardiotoxicity that is mediated by the secretion of inflammatory factors (IL1 $\beta$ and IL8) from the lung (Skardal et al., 2017). Furthermore, the model was expanded into a six-tissue MOOC with organoids of 
endothelium, testis, and brain, and tissue interaction was demonstrated using the prodrug ifosfamide, which, upon hepatic bioactivation to chloroacetaldehyde, significantly increased neurotoxicity in the brain compartment (Rajan et al., 2020). Although these data are encouraging, the high concentrations of ifosfamide used ( $1 \mathrm{mM})$ are of concern with regard to result translation. Using a similar approach, organotypic cultures of brain, pancreas, liver, lung, heart, gut, and endometrium were integrated to study effects of the COMT inhibitor tolcapone, a drug used for the treatment of Parkinson disease (Wang et al., $2019 b)$. It was confirmed by mass spectrometry that tolcapone was metabolized into at least 12 phase I and phase II metabolites and that therapeutically relevant concentrations caused metabolic reconfiguration of the 3D neural constructs comprised of diverse neuronal and glial populations, providing mechanistic support for tolcapone action at the molecular level. However, the relevance of the other tissue models remains to be confirmed.

Besides interactions of "healthy" tissue models, there is considerable interest in evaluating the effects of hepatic drug metabolism on cancer cell survival. Hepatic cell models (HepaRG or HepG2 cell lines) were shown to activate the antineoplastic prodrugs capecitabine and tegafur to their active metabolite 5fluorouracil, which in turn reduced the viability of HCT-116 cancer cells in the downstream compartment (Satoh et al., 2017; LaValley et al., 2021). In a circulating fluidic system, a hepatic compartment was connected to a glioblastoma multiforme chamber (M059K cells), and the system was perfused with either temozolomide or ifosfamide (Ma et al., 2012). Hepatic metabolism of temozolomide reduced its M059K cytotoxicity, whereas metabolic activation of ifosfamide increased cancer cell death. Similar effects were demonstrated for the effect of luteolin on HeLa cells (Lee et al., 2017b) and the bioactivation of the antineoplastic prodrug irinotecan (Shinha et al., 2020). In a three-tissue constellation with recirculation, liver and intestinal models were cultured together with lung cancer cells (Kimura et al., 2015). The device consisted of a porous collagen-coated polyester membrane separating the luminal and portal side of the intestinal compartment used for Caco-2 culture serially connected to a downstream liver (HepG2 cells) and cancer compartments (A549 cells). Application of prodrugs cyclophosphamide or irinotecan to the system reduced viability of the cancer compartment independent of Caco-2 cells, whereas cytotoxicity was reduced if the liver compartment was omitted.

In addition to their application in toxicity studies, coculture models have been used to measure systemic pharmacokinetics by coupling liver models and intestinal transwell cultures. Caco-2 cells in a perfused coculture chip maintain barrier function and absorption of propranolol, mannitol, and caffeine, whereas HepG2 in the same device retain urea or albumin secretion (Esch et al., 2016; Chen et al., 2018). Using diclofenac and hydrocortisone as model compounds, the integrated chip could be used to derive parameters for intestinal permeability and hepatic clearance, the latter of which differed significantly from the respective monoculture (Tsamandouras et al., 2017). Furthermore, intestine-liver coculture could recapitulate the extensive metabolism of apigenin in both intestine and liver (Choe et al., 2017).

Besides toxicity and pharmacokinetics, MOOCs have been used for the study of a multitude of diverse applications, including the investigation of signaling along the gut-brain axis (Raimondi et al., 2019; Trapecar et al., 2021), modeling of metastasis formation (Liu et al., 2019), and the control of glucose metabolism and homeostasis (Bauer et al., 2017; Lee et al., 2019).

Overall, these developments to reconstruct a "humanon-a-chip" are promising and exciting, but care must be taken not to overstate results. Most MOOC studies continue to use cell lines and 2D cultures to model the individual tissue constituents. We, however, argue that such integrated microphysiological models are only as strong as the weakest link, and thus, particular emphasis has to be given to the careful characterization of the phenotypes and functions of the individual tissue modules. Only then will it be possible to convert the increased technical complexity of MOOCs into improved models of pharmacokinetics and toxicity.

\section{Bioprinting as a Novel Modality for the Generation of Organotypic Cultures}

Spheroid culture, microfluidic chips, and organoids have provided important contributions to our ability to emulate native tissue function in an in vitro setting. However, they do not precisely recapitulate the microarchitecture of the native tissue. To this end, 3D bioprinting has emerged as an important modality that allows the precise deposition of cells and extracellular factors to create complex cellular constructs (Heinrich et al., 2019). Bioprinting applications can be subdivided into 1) the printing of biocompatible cell-free constructs, such as prostheses and vascular stents, and 2) the bioprinting of tissue constructs in which live cells are deposited together with signaling molecules and scaffolding factors to produce biomimetic 3D tissue constructs. Because of their relevance for drug discovery and development, we will focus in this review on the latter.

$3 \mathrm{D}$ bioprinting starts with the design of the tissue construct to be printed; templates can be generated de novo or be based on data acquired using various imaging techniques, such as magnetic resonance imaging or computed tomography. The tissue design in turn determines the printing strategy and choice of 

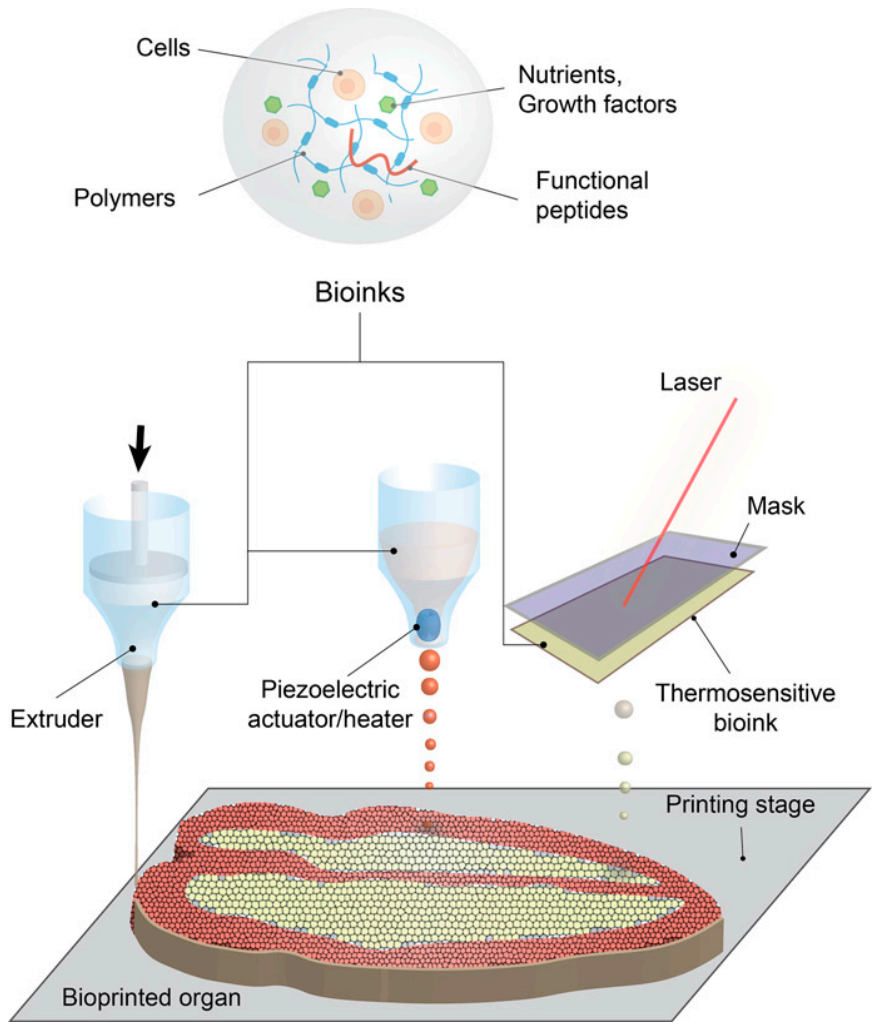

Fig. 9. Schematic depiction of bioprinting technology. The cells to be printed are embedded in a biocompatible matrix comprising polymers, nutrients, growth factors, and functional peptides jointly termed bioink. This solution can be deposited with high spatiotemporal resolution using different methodologically distinct modalities. In extrusion-based bioprinting (left), the cell-laden bioinks are deposited as continuous streams, whereas droplet-based bioprinting (middle) partitions the bioink into individual microdroplets. In contrast to both of these methods, laserassisted bioprinting (right) deposits cells using a nozzle-free approach in which the bioink is locally heated using a UV laser and then drops onto the printing stage.

bioink, of which alginate, gelatin, and gelatin-methacryloyl are the most common (Gungor-Ozkerim et al., 2018; Mancha Sánchez et al., 2020). In the following, we classify the main methodological strategies for the bioprinting of cell-laden bioinks into extrusion-based, droplet-based, and light-assisted bioprinting methods and discuss their main advantages and limitations for the fabrication of human organotypic tissue constructs (Fig. 9; Table 8).

TABLE 8

Advantages and disadvantages of bioprinting methods

\begin{tabular}{lccc}
\hline & $\begin{array}{c}\text { Extrusion- } \\
\text { Based }\end{array}$ & $\begin{array}{c}\text { Droplet- } \\
\text { Based }\end{array}$ & $\begin{array}{c}\text { Laser- } \\
\text { Assisted }\end{array}$ \\
\hline Cell viability & $50 \%-70 \%$ & $80 \%-90 \%$ & $95 \%$ \\
Printing precision & + & +++ & ++ \\
Printing resolution & $100-200 \mu \mathrm{m}$ & $30-50 \mu \mathrm{m}$ & $10 \mu \mathrm{m}$ \\
Throughput & + & +++ & ++ \\
Cell density & $10^{8} \mathrm{cells} / \mathrm{ml}$ & $<10^{6} \mathrm{cells} / \mathrm{ml}$ & $<10^{6} \mathrm{cells} / \mathrm{ml}$ \\
Bioink viscosity & $30-10^{7} \mathrm{mPa}$ & $3-12 \mathrm{mPa}$ & $1-300 \mathrm{mPa}$ \\
Single-cell deposition & $\mathrm{No}$ & Inconsistent & Yes \\
Possibility to print & Yes & Yes & No \\
scaffold-free & & & Medium \\
Cost & Low & High \\
Commercial availability & +++ & ++ & + \\
\hline
\end{tabular}

\section{A. Extrusion-Based Bioprinting}

Extrusion-based bioprinting is the most widely used bioprinting method, at least in part due to its versatility and relatively low costs. Using this technique, cell-laden bioinks are deposited as streams that are continuously extruded from a nozzle, as compared with individual droplet deposition seen with other methods (Ozbolat and Hospodiuk, 2016). Bioink can be dispensed using compressed air or mechanically using a piston or screw. Pneumatic extrusion is suitable for hydrogels with shear-thinning property, whereas the mechanic methods are more applicable for high viscosity or semisolid biomaterials and allow for superior volumetric control, resulting in higher printing resolution. However, more thorough cleaning and disinfection protocols are required when using these methods compared with pneumatic extrusion.

Extrusion-based methods allow for the printing of structures with high cell density and have been successfully used to print skin, nerves, liver, skeletal muscle, and endothelial constructs for drug screening and development as tumor models for patho-mechanistic studies and for applications within regenerative medicine. Yet, extrusion printing is slow and limited to relatively small tissues. Furthermore, it has comparatively low resolution of $100-200 \mu \mathrm{m}$ and exerts considerable shear stress resulting in low cell viability. Although these effects can be mitigated by increased cellular encapsulation in the bioink (Hiller et al., 2018), extrusion-based printing is nevertheless not suitable for the printing of sensitive cell types, which includes most primary cells.

Another novel approach is the integrated tissue-organ printing (ITOP) system in which mechanical stability is imparted by coprinting patterned biodegradable polymers and sacrificial hydrogels. As diffusion limits pose constraints for the dimension of bioengineered tissue construct, microchannels are incorporated into ITOP cell constructs, facilitating nutrient and oxygen diffusion. The ITOP system has produced skeletal muscle tissue exhibiting responses to electrical stimulation similar to those of immature, developing muscle (Kang et al., 2018). Resulting muscle constructs displayed native-like ultrastructural organization and improved viability, myosin heavy chain myofiber area, $\alpha$-sarcomeric actin area, and laminin area when compared with nonprinted cells (Kim et al., 2018). However, although tissue construct architectures can be precisely reconstructed, careful electrophysiological and functional analyses have not yet been conducted. In an interesting study, $\mathrm{PHH}$, primary stellate cells, and HUVEC cells (as model for the liver sinusoidal endothelium) were printed (Nguyen et al., 2016). The resulting tissue constructs remained viable for multiple weeks in culture and could detect hepatotoxicity of trovafloxacin at therapeutically relevant concentrations. Furthermore, 
the model was able to mimic TGF $\beta$ and methotrexateinduced fibrinogenesis (Norona et al., 2019). Interestingly, hepatic phenotypes in this model improved after 1 week of culture, likely because of delayed recovery from the considerable cell stress of the printing process.

\section{B. Droplet-Based Bioprinting}

Droplet-based bioprinting constitutes an umbrella term for approaches in which cells are deposited as cell-laden bioink droplets at predefined locations. Formation of droplets can be accomplished using inkjet bioprinting and electrohydrodynamic jetting. Inkjet bioprinting was the first bioprinting technique to be presented (Klebe, 1988). The modality can be further subdivided into continuous inkjet printing that uses a stream of electrically conductive bioinks that separate into individual droplets due to Rayleigh instability, thus allowing guidance by electrical or magnetic fields and drop-on-demand (DOD) inkjet printing that has lower drop-generating frequency but results in the formation of smaller drop volumes and thus higher printing resolution. Notably, the use of DOD is much more common since continuous inkjet printing is associated with a high risk of contamination because of bioink recirculation. In DOD printing, droplets are mostly generated by localized short heating that results in the results in the formation of a small vapor pocket, which is sufficient to push a droplet of bioink from the nozzle. Alternatively, drops can be generated using piezoelectric or microelectromechanical system actuators that reversibly deform a crystal or membrane to eject a bioink droplet (Murphy and Atala, 2014; Gudapati et al., 2016).

In contrast to inkjet printing, electrohydrodynamic jetting works by generating an electric field between nozzle and substrate that results in electrostatic repulsions of the ions within nozzle and eventual droplet release. Thereby, this method circumvents heating or excessive harmful pressure on the cells and is hence most suitable when using small orifices, high cell concentrations, or large weight-to-volume ratios (Onses et al., 2015).

Advantages of droplet-based bioprinting include the relatively low cost and simplicity of the technical setup as well as the higher throughput and increased precision compared with other modalities, allowing resolutions down to $2 \mu \mathrm{m}$ for hydrogels and $30-50 \mu \mathrm{m}$ for cells. Droplet-based bioprinting does not impair ESC or iPSC pluripotency and is compatible with HLC differentiation postprinting (Faulkner-Jones et al., 2015). However, as a downside of the deposition of individual droplets, the method is limited to the printing of constructs with low cell density.

\section{Laser-Assisted Bioprinting}

Laser-assisted bioprinting (LAB) comprises a set of methods in which cells and substrates are deposited with high spatiotemporal resolution without the need to pass through a nozzle. In laser-induced forward transfer (LIFT) bioprinting, a UV laser locally heats an energy-absorbing layer commonly made of titanium or gold, which is coated with a film of thermosensitive cell-laden bioink. Upon exposure of the absorbing layer to a focused laser pulse, the material heats up, resulting in localized microbubble formation, which liquefies the bioink and results in the formation of a droplet falling onto the collection substrate. Various modifications of LIFT, such as absorbing film-assisted LIFT, matrixassisted pulsed laser evaporation direct writing, biologic laser processing, and others have been presented that include modifications to absorbing layer, laser configuration, and the vapor pressure of the solvent, discussion of which would go beyond the scope of this review (Guillotin et al., 2013).

The main advantage of LAB is that the cells are not subjected to shear stress during the printing process, which translates into substantially higher cell viability postprinting compared with nozzle-based methods. Furthermore, LAB avoids issues related to clogging of the nozzle by bioinks, which constitutes a common practical problem in extrusion and droplet-based bioprinting. LAB has a high speed with up to 5000 laser pulses per second and superior resolution down to single cells (Guillotin et al., 2010). However, its use and more widespread dissemination is limited by the substantial investment costs for the printer. Furthermore, phototoxicity can be problematic depending on the thickness and composition of the absorbing layer, and, as dropletbased bioprinting, laser-assisted bioprinting is only applicable to the printing of constructs with relatively low cell densities.

\section{Technical Considerations for Culture Devices}

\section{A. Materials for Culture Vessels}

The selection of appropriate materials for the design and fabrication of culture systems is of great importance. Depending on the intended applications, the properties of materials in terms of biocompatibility, drug absorption, oxygen permeability, autofluorescence, surface energy, and mechanical robustness need to be scrutinized (Table 9).

Historically, silicon and glass were the predominant materials for organ-on-a-chip applications because of their well established knowledge base originating from their use in semiconductors and integrated circuit domains. Thanks to very-large-scale integration technology, a broad range of microfabrication and nanofabrication techniques originally developed for fabrication and integration of millions of very small transistors into silicon chips were also adopted for the fabrication of microfluidic devices. 
TABLE 9

Advantages and disadvantages of commonly used materials for microphysiological devices

\begin{tabular}{|c|c|c|c|c|c|c|}
\hline & & Glass & Silicon & Thermoplastics & Thermosets & Elastomers \\
\hline \multirow{8}{*}{$\begin{array}{l}\text { Material } \\
\quad \text { properties }\end{array}$} & Knowledge base in biology & High & Medium & Medium & Medium & Good \\
\hline & Optical transparency & High & None & Medium-good & Medium-good & Good \\
\hline & Gas permeability & Very low & Very low & Low & Low & Medium-high \\
\hline & Mechanical robustness & High & Good & Good-high & Good-high & Low \\
\hline & Tunable mechanical property & Low & Low & Medium & Medium-good & Low \\
\hline & Chemical leachability & None & None & Low-medium & Low-medium & High \\
\hline & $\begin{array}{l}\text { Hydrophobic molecule } \\
\text { sorption }\end{array}$ & Very low & Very low & Low-medium & Low-medium & High \\
\hline & Cell attachment & Very high & High & Medium-high & Medium-high & Low-medium \\
\hline \multirow[t]{5}{*}{$\begin{array}{l}\text { Manufacturing } \\
\text { properties }\end{array}$} & Common fabrication methods & Photolithography & $\begin{array}{c}\text { Photolithography, } \\
\text { E-beam } \\
\text { lithography }\end{array}$ & Injection molding & $\begin{array}{l}\text { Soft lithography, } \\
\text { reaction injection } \\
\text { molding }\end{array}$ & Soft lithography \\
\hline & Ease of rapid prototyping & Very low & Low & Low & Medium-high & Medium-high \\
\hline & $\begin{array}{c}\text { Cost of prototyping (material } \\
\text { and fabrication) }\end{array}$ & Very high & High & Medium & Low-medium & Low \\
\hline & $\begin{array}{l}\text { Industrial microstructuring } \\
\text { capability }\end{array}$ & High & High & High & Medium-high & Low \\
\hline & Scalability & Medium-high & Medium-high & High & Good & Low \\
\hline
\end{tabular}

Silicon and glass are both biocompatible with well known surface chemistry protocols and therefore promising for cell-culture applications (Table 9). Nevertheless, complex processing, high cost of fabrication, and mechanical rigidity associated with these materials put severe limitations on their widespread use in biomedical applications. Additionally, silicon is opaque, and therefore, its compatibility with optical imaging is limited. Polymers, on the other hand, offer a wider range of mechanical rigidity and can be manufactured more cost-efficiently. Polymers are generally categorized into three main classes: elastomers, thermoplastics, and thermosets. Below, we provide a short description of these polymers and describe their properties with respect to the biologic applications. We also present some examples of polymer materials that are commonly used for microfluidic and organ-on-a-chip applications.

1. Elastomers. Elastomers are characterized by macromolecular structures with linear chains of high flexibility and few crosslinks, resulting in elastic and rubbery properties. Notably, chain elongation in elastomeric networks is reversible. The rather low cost of fabrication in combination with its facile use have made PDMS favorable for rapid prototyping. Moreover, PDMS offers a biocompatible, gas-permeable, and transparent surface, rendering this material suitable for cell-based applications. In addition to its common use as a bulk microfluidic material, the gas permeability of PDMS has also enabled its use for other innovative applications, such as the integration of embedded pumps or valves. In these applications, a thin layer of PDMS membrane can be embedded into a system to control and manipulate fluid inside microchannels (Eddings and Gale, 2006). Such approaches reduce the need for the addition of external materials that are prone to introduce contaminations into the system. PDMS membranes can further be harnessed for filtering applications as a more facile and cost- effective alternative to silicon membranes (Nam et al., 2019).

Despite such advantages, however, PDMS suffers from considerable drawbacks. PDMS strongly absorbs hydrophobic molecules, which hinders the use of PDMS devices for pharmacological and toxicological studies. In addition, leached monomers from bulk to the surface and medium can affect cellular phenotypes. Furthermore, the hydrophobic surface of PDMS often needs to be modified to allow for a smoother flow in microfluidic devices, particularly for narrow channels. Such surface modifications are often not durable, which in turn leads to restoring the original surface energy of this material.

To overcome the aforementioned limitations, the development of alternative elastomeric materials, such as poly(itaconate-co-citrate-co-octanediol) (Davenport Huyer et al., 2019), petrafluoroethylene-propylene (Sano et al., 2019), and poly(octamethylene maleate (anhydride) citrate) (Zhao et al., 2019), is ongoing. These efforts aspire to provide a more diverse range of flexible materials. However, further research and characterization efforts are required before these polymers can replace PDMS in organ-on-a-chip and tissue engineering applications.

2. Thermoplastics. Thermoplastics are a class of polymers that can be reversibly shifted from a solid to a liquid state and vice versa by altering the temperature below melting temperature (to melt) or above the glass transition temperature (to stiffen). Thermoplastics are linear or branched and not covalently crosslinked, resulting in stiff mechanical properties that enable solid support for microfluidic devices. Notably, thermoplastic materials often feature hydrophobic surfaces and are prone to swelling when subject to organic solvents. Although the use of thermoplastic materials is currently not as prevalent as PDMS, partly due to the need for complex processing and lack of oxygen permeability, the paradigm is slowly shifting in their favor, particularly due to the high degree of drug 
absorption correlated of PDMS. For instance, a multilayer PMMA chip with an integrated electrospun membrane in poly-L-lactide was developed for the culture of human endothelial cells (Chuchuy et al., 2021). The study showed a considerably lower absorption of hydrophobic molecules into PMMA chips compared with their PDMS counterparts. Similarly, a multilayer PMMA microfluidic chip containing a thermoplastic polyurethane membrane provided enough flexibility to form on-chip microvalves and micropumps with increased chemical resistance.

Polycarbonate (PC) is another well known low-cost thermoplastic material that has raised the interest in its off-the-shelf use in microfluidic devices. The ease of fabrication is an enabler for the rapid prototyping of PC-based microfluidic devices. Additionally, the gas impermeability of PC can leverage its application when precise control over oxygen concentration is needed. For instance, PC-based microfluidic devices have been used to enable oxygen sensing in a gastrointestinal human microphysiological system (Shah et al., 2016). Furthermore, PC is commonly used as material for integrated porous membranes (Novo et al., 2017; Wang et al., 2017a; Bai et al., 2021).

Polystyrene (PS) is another thermoplastic material with well established knowledge base in biology that is routinely used for standard $2 \mathrm{D}$ cell-culture formats. Furthermore, PS plates are more recently used for 3D cell culture after ULA coating and, because of the extensive knowledge available with respect to the cell culture, PS has also been leveraged for numerous applications in perfused microfluidic devices. Cyclic olefin polymers and copolymers are other examples of thermoplastic materials that have been used for microphysiological systems. Excellent optical properties, suitability for molding and very low rates of impurity have rendered them ideal candidates for organ-on-a-chip applications (Nunes et al., 2010). Nevertheless, the high costs of these materials and their availability mostly in the form of polymer granules have mostly limited their use to commercial applications. In addition to these prominent thermoplastics, multiple alternatives have been proposed for microfluidic devices, including polyether ether ketone (Song et al., 2004; Ildefonso et al., 2012) and polysulfone (Jiang et al., 2015). These materials are cheaper and universally compatible with organic solvents and, in the case of polysulfone, offer excellent hemocompatibility for applications involving perfusion of blood.

3. Thermosets. Thermosets are a class of polymers that undergo irreversible polymerization. Prior to polymerization, thermosets are in the form of one- or often two-component liquid resins. After monomer mixing and polymerization, thermosets form covalent crosslinks, which in turn imparts chemical and thermal stability to the material. SU-8, polyurethane, and polyimide are some examples of thermosetting polymers frequently used for microphysiological systems. SU-8 has established itself as a standard resin for microelectromechanical system applications because of its uniquely high thickness (up to hundreds of microns) while also offering a high lateral resolution that enables the formation of high-aspect-ratio features. Moreover, the high crosslink density and stability when exposed to high temperature and harsh chemicals have rendered it favorable for microfabrication processing. Although SU-8 is commonly used for the fabrication of master molds in soft lithography, its biocompatibility and high mechanical stability have also opened directed uses in medical device fabrication (Nemani et al., 2013).

A more recent generation of thermosetting materials based on thiol-ene chemistry has also been presented. Off-stoichiometry thiol-ene (Carlborg et al., 2011) and thiol-ene-epoxy (Carlborg et al., 2014) are formed via click-chemistry reactions occurring in one and two polymerization stages, respectively. This class of polymers is specifically tuned for microfluidic and laboratory-on-a-chip applications, offering unique features, such as robust covalent bonding (Sandström et al., 2015; Zandi Shafagh et al., 2017), in situ surface energy patterning (Zandi Shafagh et al., 2019), and direct biofunctionalization (Zandi Shafagh et al., 2018). Given these advantages, the use of thermosetting materials for the fabrication of microfluidic devices is on the rise.

\section{B. Fabrication Techniques}

1. Photolithography. Photolithography is a standard process through which a pattern, with microfeatures and nanofeatures is transferred from a photomask onto a substrate, often by means of exposure to UV light (Chen et al., 2012). In this process, polymers, referred to as photoresists, are often used as sacrificial layers for the pattern transfer into a solid substrate, such as silicon or glass. In contrast, for some specific applications, such as fabrication of master molds for PDMS replication, the polymer remains intact after UV exposure. For instance, SU8 is a negative resist that covalently crosslinks upon exposure to UV light, often without subsequent etching. Because of the high thickness of SU-8 resist reaching hundreds of microns while having micron and submicron lateral resolution, high-aspect-ratio structuring of this material is feasible (Dervisevic et al., 2020). The use of photolithography has been commonly used for organ-on-a-chip applications, for instance for the photopatterning of silicon substrates and SU-8 to form 3D structured membranes mimicking intestinal villi (Esch et al., 2012).

Photolithography is by far the most common microfabrication method for creating molds and stamps for organ-on-a-chip systems. Nevertheless, photolithography 
is a complex and labor-intensive process that needs to be conducted inside standard cleanroom facilities. This often leads to the lack of scalability and the high cost of fabrication that is not favorable for biomedical applications.

2. Soft Lithography. Soft lithography is a well established method for the facile fabrication of microfluidic devices in academic settings (Xia and Whitesides, 1998). This is mostly originated from the low tooling costs required for the processing that allows for easy prototyping. Soft lithography is mainly based on casting PDMS onto a mold followed by subsequent heat curing and demolding, which results in the high-resolution replication of features onto a PDMS replica. Most microfluidic organ-on-a-chip devices are at least partially produced by soft lithography (Kim et al., 2012; Lee et al., 2013a, 2017b; Ahmad et al., 2014; Rigat-Brugarolas et al., 2014; Zhang et al., 2016b). Although soft lithography is the most common replication method in academic research laboratories, it is not commonly used in commercial settings because of the lack of scalability and long cycle time.

3. Microinjection Molding. Injection molding (IM) has been recognized as one of the most versatile techniques for the fabrication of polymer parts and devices. Microinjection molding ( $\mu \mathrm{IM}$ ) was further established for the fabrication of microfluidic devices (Yang et al., 2013). The scalability of $\mu \mathrm{IM}$ renders it suitable for the large-scale production of polymer devices for biomedical applications, which is of prime importance for applications, such as point-of-care diagnostics requiring disposable and cost-effective chips. Although $\mu \mathrm{IM}$ is a well established fabrication method for industrial production, its use in the academic domain is still limited mainly due to its complex processing conditions.

\section{Reaction Injection Molding. Reaction injection} molding (RIM) is based on the injection of two-component thermosetting resins in an enclosed molding setting (Heckele and Schomburg, 2003). Due to the low viscosity of resins, there is a higher replication fidelity associated with RIM compared with that of IM. UV curing systems have facilitated the use of RIM for the fabrication of microcomponents and nanocomponents as well as microfluidic devices (Sandström et al., 2015). In addition, RIM of microwell arrays with defined surface energy for applications in digital assays was reported (Zandi Shafagh et al., 2019). RIM has great potential for biomedical applications both in academic research and on industrial scales.

5. Nanoimprint Lithography. Nanoimprint lithography (NIL) is a stamp-based approach for the replication of high-resolution features from a stamp onto a substrate (Guo, 2007). NIL can be used either for direct pattern transfer implemented for end applications or followed by subsequent etching through the substrate material. NIL enables high-resolution and cost-effective manufacturing of micropatterned and nanopatterned surfaces and has found great potential for applications in life sciences. For instance, we recently developed a method for facile nanoimprinting of high-aspect-ratio nanotopographies, which modulate the mechanosensing of human hepatic cells and provide precise spatial control of cell attachment (Zandi Shafagh et al., 2020). NIL has also been implemented for the fabrication of microfluidic and nanofluidic devices with nanochannels (Sun et al., 2016). Although the capability of NIL for manufacturing of organ-on-a-chip devices is still not fully realized, the cost effectiveness and scalability of roll-to-roll nanoimprinting can pave the way for high-throughput replication of such devices (Stuart and Chen, 2009).

6. Electron Beam Lithography. Electron beam lithography (EBL) is a maskless nanopatterning technique that can be used for the direct writing of nanotopologies into an e-beam resist (Chen, 2015). EBL enables ultra-highresolution fabrication of nanofeatures with high precision and low structural collapse and has been used for highresolution patterning of biomolecules on nanopatterned surfaces that allows for spatially refined biofunctionalization of polymer substrates (Zandi Shafagh et al., 2018). Furthermore, direct protein inactivation through exposure to electron beams can be used to guide cell attachment, resulting in cell shapes constrained to the EBL-written pattern (Glezos et al., 2002; Rundqvist et al., 2007). In addition to surface patterning, EBL has been used for the fabrication of various microfluidic units, including microchannels and micromixers (Mali et al., 2006). However, because of the costs and low availability of e-beam infrastructure, the use of EBL for organ-on-a-chip devices remains in its infancy.

\section{Conclusions and Perspectives}

There has been tremendous progress in the development of organotypic and microphysiological 3D human models of tissues with relevance for drug response and toxicity. These activities have resulted in a multitude of methodologically distinct culture paradigms, including spheroid cultures, transwell cultures, MPCCs, and perfused microchips. Furthermore, organoid technology has found its way into drug testing applications, particularly for brain, intestine, and kidney cultures, whereas its use for pharmacological and toxicological applications involving liver, skeletal muscle, and heart is lagging behind.

For hepatic applications, the use of primary human liver cells constitutes by now the gold standard and has mostly replaced cell lines for studies of hepatotoxicity, pharmacokinetic profiling, and liver disease modeling. Stem cell-derived HLCs remain of interest for drug developers, particularly because of their ability to retrospectively mimic patient-specific drug hepatotoxicity (Choudhury et al., 2017)); however, their immature 
phenotype has so far precluded more widespread use. Spheroid culture has asserted itself as the predominant method for hepatotoxicity studies, whereas MPCCs remain the most commonly used paradigm for the prediction of hepatic clearance. For liver disease modeling, the methodological landscape is more heterogeneous, and promising data have been presented for spheroids and MPCCs as well as microphysiological liver systems. Hepatic organoids are interesting tools for studies of liver developmental biology, regenerative medicine, and the modeling of congenital liver diseases (Prior et al., 2019). However, their use for hepatotoxicity testing and clearance predictions as well as drug discovery for metabolic or infectious liver diseases remains limited.

Primary cells also remain the most well established bona fide cell model for analyses of nephrotoxicity and renal clearance of the proximal tubule. Due to the paucity of primary cells for other regions of the nephron, including glomerulus, distal tubule, and collecting duct, stem cell-derived microfluidic chip cultures or organoids have become predominant for their analysis. However, although promising data have been presented, careful benchmarking to in vivo phenotypes and large-scale evaluation of the predictive accuracy of these stem cell-derived models remain mostly lacking.

In contrast to liver and kidney, the predominant cell model for intestinal drug absorption and toxicity studies in either static transwell or perfused microchips remains the Caco- 2 cell line. The reason for the continued use of these cells is that they can differentiate into enterocyte-like cells and form intact monolayers with high TEER. Furthermore, the addition of flow and peristalsis-like movements results in the formation of villi structures and the emergence of enteroendocrineand mucus-producing Goblet-like cells, thus approximating the cellular complexity of the intestinal epithelium. Static transwell cultures remain the method of choice for the prediction of intestinal permeability, particularly of lipophilic compounds that primarily use the transcellular route. Intestinal perfusion chips and organoids constitute the model of choice for interaction studies with the microbiome and for modeling of intestinal diseases, particularly inflammatory bowel syndrome, and are expected to play a major role in the development of nutraceuticals.

Due to the inaccessibility of the brain, organotypic models of the cerebrum and the BBB rely primarily on pluripotent stem cells. However, given the complexity of the tissue to be modeled, it might be of little surprise that the development and characterization of brain organoids for pharmacological and toxicological applications remain in their infancy. Few studies have evaluated drug permeability and neurotoxicity; although these results provide promising proof of concept, more extensive tests using larger panels of positive and, importantly, negative control compounds are required to evaluate whether these systems are fit for purpose. In contrast, brain organoids from patient-derived iPSCs have provided important contributions to the understanding of genetic and infectious diseases, which might provide useful tools for target identification and lead optimization.

Much progress has been made in recent years in the development of cardiomyocyte models. For organotypic cultures of the heart, iPSC-derived cardiomyocytes have largely superseded the use of shortlived ex vivo cultures. These trends are fueled by the ability to metabolically purify iPSC-derived cardiomyocytes by glucose deprivation (Tohyama et al., 2013) and are expected to continue as spheroids and scaffold-based methods in which EHT aggregate around micropillar and over time form compact, structurally accurate, and functional 3D tissue. Notably, 3D cultures of cardiomyocytes are compatible with electrophysiological readouts in high-throughput formats and have already been used for the testing of drug-induced cardiac toxicity with reasonably high predictive accuracy. Furthermore, EHTs have been used to investigate the mechanisms underlying cardiac hypertrophy and fibrosis as well as IRI and provide interesting opportunities for the development of translatable therapeutic approaches.

Organotypic human skeletal muscle models have been developed using a similar set of methods and approaches as what has been developed for cardiomyocytes. However, although iPSCs are also used, the most common cell model for skeletal muscle culture is based on primary human myoblasts from needle biopsies, surgical wastes, or immortalized primary skeletal muscle cells. From a pharmacological and toxicological perspective, these models are primarily used for the evaluation of myotoxicity and the development of compounds against congenital muscle diseases.

Although a diverse range of microphysiological systems has been established, the development of appropriate materials for drug discovery and development purposes has received less attention. The facile prototyping, advantageous optical properties, and biocompatibility of PDMS have resulted in the widespread adoption of this elastomer for organ-ona-chip devices. However, the significant nonspecific sorption of hydrophobic molecules to device components made from PDMS limits the utility of this material for pharmacological and toxicological studies. Additionally, the translation of PDMS devices from lowthroughput soft lithography into the industrial setting is cumbersome since casting is not scalable. Emerging alternatives, such as thermoplastics and thermosetting polymers, can be tuned for high-throughput fabrication of microfluidic devices with lower drug absorption. Bioprinting presents exciting opportunities for the reconstruction of human tissue constructs with high spatial 
resolution, but important barriers regarding both printing methods and bioinks need to be overcome before these technologies can be routinely used for primary human cells.

In summary, organotypic and microphysiological human tissue models offer exciting opportunities for drug discovery, as they promise to provide scalable physiologically and pathophysiologically relevant assays for the mechanistic profiling of candidates, often using patient-derived cells. Furthermore, it can be expected that testing of toxicity and pharmacokinetic properties in human tissue models with relevant phenotypes will improve result translation and, eventually, reduce the rate of both efficacy and safety failures in clinical stages. An interesting use case is the development and testing of antiviral candidate drugs during the COVID19 pandemic wherein organotypic cultures were extensively used to model both pulmonary and extrapulmonary effects of SARS-CoV-2 infection and have majorly contributed to the rapid progress of repurposing programs (Youhanna et al., 2021).

Although the focus of this review is on organotypic and microphysiological models from human cells, similar cultures can be established using material from preclinically relevant animal models. Such animal cell-based models can be directly benchmarked against the corresponding in vivo model to assess how well the phenotype of interest is translated into the in vitro setting. This is particularly useful for inaccessible tissues, such as brain, where similar comparisons are difficult to conduct using human cells. Furthermore, organotypic and microphysiological animal models can provide useful insights into the species specificity of observed effects, thereby increasing confidence in the validity of human model results. Examples are the use of liver spheroids (Vorrink et al., 2018), MPCCs (Aleo et al., 2019), or microphysiological chips (Novik et al., 2017) to reveal the species specificity of compound hepatotoxicity or the comparative analysis of cisplatin nephrotoxicity in kidney cells from rat, rabbit, dog, and cat (Katayama et al., 2011).

Despite the advances, there remain, however, important limitations that need to be overcome. Currently, the field is highly prolific and driven primarily by academia and small biotech start-ups. Although the resulting methodological diversity is to be welcomed, thorough benchmarking and validation efforts are needed at the level of cell models, culture platforms, and materials, ideally conducted in a cross-sectoral multicenter setting to identify the most suitable systems for a given application. This is particularly true for iPSC-derived models, in which variations between iPSC lines regarding differentiation potential and genomic aberrations as well as donor demographics can be extensive and add further heterogeneity (Kyttälä et al., 2016; Carcamo-Orive et al., 2017; Kilpinen et al., 2017; Vigilante et al., 2019). Model evaluations should include sufficiently large sets of well accepted reference drugs so that firm conclusions on test accuracy can be drawn. Furthermore, it is critical to align the development of preclinical systems with drug developer needs to facilitate model acceptance and dissemination. To this end, the International Consortium for Innovation and Quality in Pharmaceutical Development has provided a series of position statements in which the preferred characteristics and features for microphysiological models are described to guide both system developers and end users (Baudy et al., 2020; Fabre et al., 2020; Fowler et al., 2020; Peters et al., 2020; Peterson et al., 2020; Phillips et al., 2020; Pointon et al., 2021).

We furthermore recommend efforts toward the standardization of assays, endpoints, and result reporting to facilitate cross-study comparability. One prototypic example for the transition of an organotypic model from exploratory research to regulatory acceptance is the 3D model of the skin that, upon extensive validation and standardization, has been incorporated into the Organization for Economic Cooperation and Development guidelines for skin corrosion (https://www.oecd-ilibrary. org/docserver/9789264264618-en.pdf?expires=161951203 $5 \&$ id=id\&accname=guest\&checksum=DB0E9285BFBF 07A024635F122A67F485) and skin irritation (https:// www.oecd-ilibrary.org/docserver/9789264242845-en.pdf? expires $=1619512003 \&$ id $=i d \&$ acc $n$ ame $=$ guest $\&$ checksum=D1691772B286FD69660DB63B4ED9E356). We thus suggest using these successful developments as a blueprint to catalyze the effective integration of other organotypic tissue cultures into the preclinical testing pipeline. In turn, this will generate the necessary data to support informed decision-making by regulators to facilitate the increasing acceptance of organotypic and microphysiological human tissue models for translational pharmacology and toxicology.

\section{Authorship Contributions}

Wrote or contributed to the writing of the manuscript: Youhanna, Kemas, Preiss, Zhou, Shen, Cakal, Paqualini, Goparaju, Zandi Shafagh, Lind, Sellgren, Lauschke.

\section{References}

Abilez OJ, Tzatzalos E, Yang H, Zhao M-T, Jung G, Zöllner AM, Tiburcy M, Riegler J, Matsa E, Shukla P et al. (2018) Passive stretch induces structural and functional maturation of engineered heart muscle as predicted by computational modeling. Stem Cells 36:265-277.

Abud EM, Ramirez RN, Martinez ES, Healy LM, Nguyen CHH, Newman SA, Yeromin AV, Scarfone VM, Marsh SE, Fimbres C et al. (2017) iPSC-derived human microglia-like cells to study neurological diseases. Neuron 94:278-293.e9.

Adler M, Ramm S, Hafner M, Muhlich JL, Gottwald EM, Weber E, Jaklic A, Ajay AK, Svoboda D, Auerbach S et al. (2016) A quantitative approach to screen for nephrotoxic compounds in vitro. J Am Soc Nephrol 27:1015-1028.

Ahmad AA, Wang Y, Gracz AD, Sims CE, Magness ST, and Allbritton NL (2014) Optimization of 3-D organotypic primary colonic cultures for organ-on-chip applications. J Biol Eng 8:9.

Ahn S, Ardoña HAM, Lind JU, Eweje F, Kim SL, Gonzalez GM, Liu Q, Zimmerman JF, Pyrgiotakis G, Zhang Z et al. (2018) Mussel-inspired 3D fiber scaffolds for heart-on-a-chip toxicity studies of engineered nanomaterials. Anal Bioanal Chem 410:6141-6154.

Alassaf A, Tansik G, Mayo V, Wubker L, Carbonero D, and Agarwal A (2019) Engineering anisotropic cardiac monolayers on microelectrode arrays for noninvasive analyses of electrophysiological properties. Analyst (Lond) 145:139-149.

Aleo MD, Ukairo O, Moore A, Irrechukwu O, Potter DM, and Schneider RP (2019) Liver safety evaluation of endothelin receptor antagonists using HepatoPac ${ }^{\circledR}$ : a 
single model impact assessment on hepatocellular health, function and bile acid disposition. J Appl Toxicol 39:1192-1207.

Allende ML, Cook EK, Larman BC, Nugent A, Brady JM, Golebiowski D, SenaEsteves M, Tifft CJ, and Proia RL (2018) Cerebral organoids derived from Sandhoff disease-induced pluripotent stem cells exhibit impaired neurodifferentiation. $J$ Lipid Res 59:550-563.

Ananthanarayanan A, Nugraha B, Triyatni M, Hart S, Sankuratri S, and Yu H (2014) Scalable spheroid model of human hepatocytes for hepatitis C infection and replication. Mol Pharm 11:2106-2114.

Anderson S, Luffer-Atlas D, and Knadler MP (2009) Predicting circulating human metabolites: how good are we? Chem Res Toxicol 22:243-256.

Antunes F, Andrade F, Araújo F, Ferreira D, and Sarmento B (2013) Establishment of a triple co-culture in vitro cell models to study intestinal absorption of peptide drugs. Eur J Pharm Biopharm 83:427-435.

Aratyn-Schaus Y, Pasqualini FS, Yuan H, McCain ML, Ye GJC, Sheehy SP Campbell PH, and Parker KK (2016) Coupling primary and stem cell-derived cardiomyocytes in an in vitro model of cardiac cell therapy. $J$ Cell Biol 212:389-397.

Araújo F, Pereira C, Costa J, Barrias C, Granja PL, and Sarmento B (2016) In vitro M-like cells genesis through a tissue-engineered triple-culture intestinal model J Biomed Mater Res B Appl Biomater 104:782-788.

Araújo F and Sarmento B (2013) Towards the characterization of an in vitro triple co-culture intestine cell model for permeability studies. Int J Pharm 458:128-134.

Arez F, Rebelo SP, Fontinha D, Simão D, Martins TR, Machado M, Fischli C, Oeuvray C, Badolo L, Carrondo MJT et al. (2019) Flexible 3D cell-based platforms for the discovery and profiling of novel drugs targeting Plasmodium hepatic infection. ACS Infect Dis 5:1831-1842.

Artursson P and Karlsson J (1991) Correlation between oral drug absorption in humans and apparent drug permeability coefficients in human intestinal epithelial (Caco-2) cells. Biochem Biophys Res Commun 175:880-885.

Artursson P, Ungell AL, and Löfroth JE (1993) Selective paracellular permeability in two models of intestinal absorption: cultured monolayers of human intestinal epithelial cells and rat intestinal segments. Pharm Res 10:1123-1129.

Aurora M and Spence JR (2016) hPSC-derived lung and intestinal organoids as models of human fetal tissue. Dev Biol 420:230-238.

Awdishu L and Mehta RL (2017) The 6R's of drug induced nephrotoxicity. BMC Nephrol 18:124.

Azzimato V, Jager J, Chen P, Morgantini C, Levi L, Barreby E, Sulen A, Oses C, Willerbrords J, Xu C et al. (2020) Liver macrophages inhibit the endogenous antioxidant response in obesity-associated insulin resistance. Sci Transl Med 12:eaaw9709.

Azzimato V, Chen P, Barreby E, Morgantini C, Levi L, Vankova A, Jager J, Sulen A, Diotallevi M, Shen JX, Miller A, Ellis E, Rydén M, Näslund E, Thorell A, Lauschke VM, Channon KM, Crabtree MJ, Haschemi A, Craige SM, Mori M, Spallotta F and Aouadi M (2021) Hepatic miR-144 drives fumarase activity preventing NRF2 activation during obesity. Gastroenterology. doi:https://doi.org/ 10.1053/j.gastro.2021.08.030. Online ahead of print.

Bai J, Haase K, Roberts JJ, Hoffmann J, Nguyen HT, Wan Z, Zhang S, Sarker B, Friedman N, Ristić-Lehmann Č et al. (2021) A novel 3D vascular assay for evaluating angiogenesis across porous membranes. Biomaterials 268:120592.

Bailey J, Thew M, and Balls M (2014) An analysis of the use of animal models in predicting human toxicology and drug safety. Altern Lab Anim 42:181-199.

Bajaj P, Chung G, Pye K, Yukawa T, Imanishi A, Takai Y, Brown C, and Wagoner MP (2020) Freshly isolated primary human proximal tubule cells as an in vitro model for the detection of renal tubular toxicity. Toxicology 442:152535.

Bajaj P, Rodrigues AD, Steppan CM, Engle SJ, Mathialagan S, and Schroeter T (2018) Human pluripotent stem cell-derived kidney model for nephrotoxicity studies. Drug Metab Dispos 46:1703-1711.

Ballabh P, Braun A, and Nedergaard M (2004) The blood-brain barrier: an overview: structure, regulation, and clinical implications. Neurobiol Dis 16:1-13.

Ballard TE, Wang S, Cox LM, Moen MA, Krzyzewski S, Ukairo O, and Obach RS (2016) Application of a micropatterned cocultured hepatocyte system to predict preclinical and human-specific drug metabolism. Drug Metab Dispos 44:172-179.

Barberi T, Bradbury M, Dincer Z, Panagiotakos G, Socci ND, and Studer L (2007) Derivation of engraftable skeletal myoblasts from human embryonic stem cells. Nat Med 13:642-648.

Baudoin R, Griscom L, Monge M, Legallais C, and Leclerc E (2007) Development of a renal microchip for in vitro distal tubule models. Biotechnol Prog 23:1245-1253.

Baudy AR, Otieno MA, Hewitt P, Gan J, Roth A, Keller D, Sura R, Van Vleet TR, and Proctor WR (2020) Liver microphysiological systems development guidelines for safety risk assessment in the pharmaceutical industry. Lab Chip 20:215-225.

Bauer S, Wennberg Huldt C, Kanebratt KP, Durieux I, Gunne D, Andersson S, Ewart L, Haynes WG, Maschmeyer I, Winter A et al. (2017) Functional coupling of human pancreatic islets and liver spheroids on-a-chip: towards a novel human ex vivo type 2 diabetes model. Sci Rep 7:14620.

Baze A, Parmentier C, Hendriks DFG, Hurrell T, Heyd B, Bachellier P, Schuster C, Ingelman-Sundberg M, and Richert L (2018) Three-dimensional spheroid primary human hepatocytes in monoculture and coculture with nonparenchymal cells. Tissue Eng Part C Methods 24:534-545.

Bazes A, Nollevaux G, Coco R, Joly A, Sergent T, and Schneider Y-J (2011) Development of a triculture based system for improved benefit/risk assessment in pharmacology and human food. BMC Proc 5 (Suppl 8):67.

Beaurivage C, Kanapeckaite A, Loomans C, Erdmann KS, Stallen J, and Janssen RAJ (2020) Development of a human primary gut-on-a-chip to model inflammatory processes. Sci Rep 10:21475.

Beaurivage C, Naumovska E, Chang YX, Elstak ED, Nicolas A, Wouters H, van Moolenbroek G, Lanz HL, Trietsch SJ, Joore J et al. (2019) Development of a gut-on-a-chip model for high throughput disease modeling and drug discovery. Int J Mol Sci 20:5661.
Bedut S, Seminatore-Nole C, Lamamy V, Caignard S, Boutin JA, Nosjean O, Stephan J-P, and Coge F (2016) High-throughput drug profiling with voltageand calcium-sensitive fluorescent probes in human iPSC-derived cardiomyocytes. Am J Physiol Heart Circ Physiol 311:H44-H53.

Bell CC, Chouhan B, Andersson LC, Andersson H, Dear JW, Williams DP, and Söderberg M (2020) Functionality of primary hepatic non-parenchymal cells in a 3D spheroid model and contribution to acetaminophen hepatotoxicity. Arch Toxicol 94:1251-1263.

Bell CC, Dankers ACA, Lauschke VM, Sison-Young R, Jenkins R, Rowe C, Goldring CE, Park K, Regan SL, Walker T et al. (2018) Comparison of hepatic 2D sandwich cultures and $3 \mathrm{D}$ spheroids for long-term toxicity applications: a multicenter study. Toxicol Sci 162:655-666.

Bell CC, Hendriks DFG, Moro SML, Ellis E, Walsh J, Renblom A, Fredriksson Puigvert L, Dankers ACA, Jacobs F, Snoeys J et al. (2016) Characterization of primary human hepatocyte spheroids as a model system for drug-induced liver injury, liver function and disease. Sci Rep 6:25187.

Bell CC, Lauschke VM, Vorrink SU, Palmgren H, Duffin R, Andersson TB, and Ingelman-Sundberg M (2017) Transcriptional, functional, and mechanistic comparisons of stem cell-derived hepatocytes, HepaRG cells, and threedimensional human hepatocyte spheroids as predictive in vitro systems for druginduced liver injury. Drug Metab Dispos 45:419-429.

Bell LN and Chalasani N (2009) Epidemiology of idiosyncratic drug-induced liver injury. Semin Liver Dis 29:337-347.

Berger DR, Ware BR, Davidson MD, Allsup SR, and Khetani SR (2015) Enhancing the functional maturity of induced pluripotent stem cell-derived human hepatocytes by controlled presentation of cell-cell interactions in vitro. Hepatology 61:1370-1381.

Bergmann S, Lawler SE, Qu Y, Fadzen CM, Wolfe JM, Regan MS, Pentelute BL, Agar NYR, and Cho C-F (2018) Blood-brain-barrier organoids for investigating the permeability of CNS therapeutics. Nat Protoc 13:2827-2843.

Bershteyn M, Nowakowski TJ, Pollen AA, Di Lullo E, Nene A, Wynshaw-Boris A, and Kriegstein AR (2017) Human iPSC-derived cerebral organoids model cellular features of lissencephaly and reveal prolonged mitosis of outer radial glia. Cell Stem Cell 20:435-449.e4.

Bersini S, Gilardi M, Ugolini GS, Sansoni V, Talò G, Perego S, Zanotti S, Ostano P, Mora M, Soncini M et al. (2018) Engineering an environment for the study of fibrosis: a 3D human muscle model with endothelium specificity and endomysium. Cell Rep 25:3858-3868.e4.

Berthiaume F, Moghe PV, Toner M, and Yarmush ML (1996) Effect of extracellular matrix topology on cell structure, function, and physiological responsiveness hepatocytes cultured in a sandwich configuration. FASEB J 10:1471-1484.

Bhogal RH, Hodson J, Bartlett DC, Weston CJ, Curbishley SM, Haughton E, Williams KT, Reynolds GM, Newsome PN, Adams DH et al. (2011) Isolation of primary human hepatocytes from normal and diseased liver tissue: a one hundred liver experience. PLoS One 6:e18222.

Bi YA, Kazolias D, and Duignan DB (2006) Use of cryopreserved human hepatocytes in sandwich culture to measure hepatobiliary transport. Drug Metab Dispos 34:1658-1665.

Bian S, Repic M, Guo Z, Kavirayani A, Burkard T, Bagley JA, Krauditsch C, and Knoblich JA (2018) Genetically engineered cerebral organoids model brain tumor formation. Nat Methods 15:631-639.

Billat P-A, Roger E, Faure S, and Lagarce F (2017) Models for drug absorption from the small intestine: where are we and where are we going? Drug Discov Today 22:761-775.

Birey F, Andersen J, Makinson CD, Islam S, Wei W, Huber N, Fan HC, Metzler KRC, Panagiotakos G, Thom N et al. (2017) Assembly of functionally integrated human forebrain spheroids. Nature 545:54-59.

Bissig K-D, Han W, Barzi M, Kovalchuk N, Ding L, Fan X, Pankowicz FP, Zhang Q-Y, and Ding X (2018) P450-humanized and human liver chimeric mouse models for studying xenobiotic metabolism and toxicity. Drug Metab Dispos 46:1734-1744

Björnsson E (2010) Review article: drug-induced liver injury in clinical practice. Aliment Pharmacol Ther 32:3-13.

Björnsson ES, Bergmann OM, Björnsson HK, Kvaran RB, and Olafsson S (2013) Incidence, presentation, and outcomes in patients with drug-induced liver injury in the general population of Iceland. Gastroenterology 144:1419-1425, 1425.e1-1425.e3, quiz e19-e20.

Bonn B, Svanberg P, Janefeldt A, Hultman Ia, and Grime K (2016) Determination of human hepatocyte intrinsic clearance for slowly metabolized compounds: comparison of a primary hepatocyte/stromal cell coculture with plated primary hepatocytes and hepaRG. Drug Metab Dispos 44:527-533.

Boudou T, Legant WR, Mu A, Borochin MA, Thavandiran N, Radisic M, Zandstra PW, Epstein JA, Margulies KB, and Chen CS (2012) A microfabricated platform to measure and manipulate the mechanics of engineered cardiac microtissues. Tissue Eng Part A 18:910-919.

Bovard D, Sandoz A, Luettich K, Frentzel S, Iskandar A, Marescotti D, Trivedi K Guedj E, Dutertre Q, Peitsch MC et al. (2018) A lung/liver-on-a-chip platform for acute and chronic toxicity studies. Lab Chip 18:3814-3829.

Brown CDA, Sayer R, Windass AS, Haslam IS, De Broe ME, D'Haese PC, and Verhulst A (2008) Characterisation of human tubular cell monolayers as a mode of proximal tubular xenobiotic handling. Toxicol Appl Pharmacol 233:428-438.

Brown JA, Pensabene V, Markov DA, Allwardt V, Neely MD, Shi M, Britt CM, Hoilett OS, Yang Q, Brewer BM et al. (2015) Recreating blood-brain barrier physiology and structure on chip: a novel neurovascular microfluidic bioreactor. Biomicrofluidics 9:054124.

Bueters R, Bael A, Gasthuys E, Chen C, Schreuder MF, and Frazier KS (2020) Ontogeny and cross-species comparison of pathways involved in drug absorption, distribution, metabolism, and excretion in neonates (review): kidney. Drug Metab Dispos 48:353-367. 
Bulutoglu B, Rey-Bedón C, Kang YBA, Mert S, Yarmush ML, and Usta OB (2019) A microfluidic patterned model of non-alcoholic fatty liver disease: applications to disease progression and zonation. Lab Chip 19:3022-3031.

Burbank MG, Burban A, Sharanek A, Weaver RJ, Guguen-Guillouzo C, and Guillouzo A (2016) Early alterations of bile canaliculi dynamics and the Rho kinase/Myosin light chain kinase pathway are characteristics of drug-induced intrahepatic cholestasis. Drug Metab Dispos 44:1780-1793.

Burridge PW, Anderson D, Priddle H, Barbadillo Muñoz MD, Chamberlain S, Allegrucci C, Young LE, and Denning C (2007) Improved human embryonic stem cell embryoid body homogeneity and cardiomyocyte differentiation from a novel V-96 plate aggregation system highlights interline variability. Stem Cells 25:929-938.

Burridge PW, Li YF, Matsa E, Wu H, Ong S-G, Sharma A, Holmström A, Chang AC, Coronado MJ, Ebert AD et al. (2016) Human induced pluripotent stem cellderived cardiomyocytes recapitulate the predilection of breast cancer patients to doxorubicin-induced cardiotoxicity. Nat Med 22:547-556.

Caiazzo M, Giannelli S, Valente P, Lignani G, Carissimo A, Sessa A, Colasante G, Bartolomeo R, Massimino L, Ferroni S et al. (2015) Direct conversion of fibroblasts into functional astrocytes by defined transcription factors. Stem Cell Reports 4:25-36.

Canals I, Ginisty A, Quist E, Timmerman R, Fritze J, Miskinyte G, Monni E, Hansen MG, Hidalgo I, Bryder D et al. (2018) Rapid and efficient induction of functional astrocytes from human pluripotent stem cells. Nat Methods 15:693-696.

Cao X, Gibbs ST, Fang L, Miller HA, Landowski CP, Shin H-C, Lennernas H, Zhong Y, Amidon GL, Yu LX et al. (2006) Why is it challenging to predict intestinal drug absorption and oral bioavailability in human using rat model. Pharm Res 23:1675-1686.

Carcamo-Orive I, Hoffman GE, Cundiff P, Beckmann ND, D'Souza SL, Knowles JW, Patel A, Papatsenko D, Abbasi F, Reaven GM et al. (2017) Analysis of transcriptional variability in a large human iPSC library reveals genetic and non-genetic determinants of heterogeneity. Cell Stem Cell 20:518-532.e9.

Carlborg CF, Haraldsson T, Öberg K, Malkoch M, and van der Wijngaart W (2011) Beyond PDMS: off-stoichiometry thiol-ene (OSTE) based soft lithography for rapid prototyping of microfluidic devices. Lab Chip 11:3136-3147.

Carlborg CF, Vastesson A, Liu Y, van der Wijngaart W, Johansson M, and Haraldsson T (2014) Functional off-stoichiometry thiol-ene-epoxy thermosets featuring temporally controlled curing stages via an UV/UV dual cure process. $J$ Polym Sci A Polym Chem 52:2604-2615.

Cashman TJ, Josowitz R, Johnson BV, Gelb BD, and Costa KD (2016) Human engineered cardiac tissues created using induced pluripotent stem cells reveal functional characteristics of BRAF-mediated hypertrophic cardiomyopathy. PLoS One 11:e0146697.

Chal J, Al Tanoury Z, Hestin M, Gobert B, Aivio S, Hick A, Cherrier T, Nesmith AP, Parker KK, and Pourquié O (2016) Generation of human muscle fibers and satellite-like cells from human pluripotent stem cells in vitro. Nat Protoc 11:1833-1850.

Chambers SM, Fasano CA, Papapetrou EP, Tomishima M, Sadelain M, and Studer L (2009) Highly efficient neural conversion of human ES and iPS cells by dual inhibition of SMAD signaling. Nat Biotechnol 27:275-280.

Chan HF, Zhang Y, and Leong KW (2016) Efficient one-step production of microencapsulated hepatocyte spheroids with enhanced functions. Small 12:2720-2730.

Chan TS, Yu H, Moore A, Khetani SR, and Tweedie D (2013) Meeting the challenge of predicting hepatic clearance of compounds slowly metabolized by cytochrome P450 using a novel hepatocyte model, HepatoPac. Drug Metab Dispos 41:2024-2032.

Chang S-Y, Weber EJ, Sidorenko VS, Chapron A, Yeung CK, Gao C, Mao Q, Shen D, Wang J, Rosenquist TA et al. (2017) Human liver-kidney model elucidates the mechanisms of aristolochic acid nephrotoxicity. JCI Insight 2:e95978.

Chang Y, Kim J, Park H, Choi H, and Kim J (2020) Modelling neurodegenerative diseases with 3D brain organoids. Biol Rev Camb Philos Soc 95:1497-1509.

Chao P, Maguire T, Novik E, Cheng KC, and Yarmush ML (2009) Evaluation of a microfluidic based cell culture platform with primary human hepatocytes for the prediction of hepatic clearance in human. Biochem Pharmacol 78:625-632.

Chatterjee S, Richert L, Augustijns P, and Annaert P (2014) Hepatocyte-based in vitro model for assessment of drug-induced cholestasis. Toxicol Appl Pharmacol 274:124-136.

Chen HJ, Miller P, and Shuler ML (2018) A pumpless body-on-a-chip model using a primary culture of human intestinal cells and a 3D culture of liver cells. Lab Chip 18:2036-2046.

Chen M, Lin Y-Q, Xie S-L, Wu H-F, and Wang J-F (2011) Enrichment of cardiac differentiation of mouse embryonic stem cells by optimizing the hanging drop method. Biotechnol Lett 33:853-858.

Chen T and Vunjak-Novakovic G (2019) Human tissue-engineered model of myocardial ischemia-reperfusion injury. Tissue Eng Part A 25:711-724.

Chen W, Lam RHW, and Fu J (2012) Photolithographic surface micromachining of polydimethylsiloxane (PDMS). Lab Chip 12:391-395.

Chen Y (2015) Nanofabrication by electron beam lithography and its applications: a review. Microelectron Eng 135:57-72.

Chen Y, Tang D, Wu H, Wu Y, Yuan T, Zhang H, Jiao Y, Yu W, and Yan H (2021) Assessment of long-term functional maintenance of primary human hepatocytes to predict drug-induced hepatoxicity in vitro. Arch Toxicol 95:2431-2442.

Cheng KC, Li C, and Uss AS (2008) Prediction of oral drug absorption in humansfrom cultured cell lines and experimental animals. Expert Opin Drug Metab Toxicol 4:581-590.

Chiou WL and Barve A (1998) Linear correlation of the fraction of oral dose absorbed of 64 drugs between humans and rats. Pharm Res 15:1792-1795.

Chiou WL and Buehler PW (2002) Comparison of oral absorption and bioavailablity of drugs between monkey and human. Pharm Res 19:868-874.
Chiou WL, Jeong HY, Chung SM, and Wu TC (2000) Evaluation of using dog as an animal model to study the fraction of oral dose absorbed of 43 drugs in humans. Pharm Res 17:135-140.

Chiron S, Tomczak C, Duperray A, Lainé J, Bonne G, Eder A, Hansen A, Eschenhagen T, Verdier C, and Coirault C (2012) Complex interactions between human myoblasts and the surrounding 3D fibrin-based matrix. PLoS One 7:e36173

Cho C-F, Wolfe JM, Fadzen CM, Calligaris D, Hornburg K, Chiocca EA, Agar NYR, Pentelute BL, and Lawler SE (2017) Blood-brain-barrier spheroids as an in vitro screening platform for brain-penetrating agents. Nat Commun 8:15623.

Choe A, Ha SK, Choi I, Choi N, and Sung JH (2017) Microfluidic gut-liver chip for reproducing the first pass metabolism. Biomed Microdevices 19:4

Choi W, Yeruva S, and Turner JR (2017) Contributions of intestinal epithelial barriers to health and disease. Exp Cell Res 358:71-77.

Choi YJ, Park J, and Lee S-H (2013) Size-controllable networked neurospheres as a $3 \mathrm{D}$ neuronal tissue model for Alzheimer's disease studies. Biomaterials 34: 2938-2946.

Choudhury Y, Toh Y-C, Xing J, Qu Y, Poh J, Li H, Tan HS, Kanesvaran R, Yu H, and Tan M-H (2017) Patient-specific hepatocyte-like cells derived from induced pluripotent stem cells model pazopanib-mediated hepatotoxicity. Sci Rep 7:41238. Chu X, Bleasby K, and Evers R (2013) Species differences in drug transporters and implications for translating preclinical findings to humans. Expert Opin Drug Metab Toxicol 9:237-252.

Chua ACY, Ananthanarayanan A, Ong JJY, Wong JY, Yip A, Singh NH, Qu Y, Dembele L, McMillian M, Ubalee R et al. (2019) Hepatic spheroids used as an in vitro model to study malaria relapse. Biomaterials 216:119221.

Chuchuy J, Rogal J, Ngo T, Stadelmann K, Antkowiak L, Achberger K, Liebau S, Schenke-Layland K, and Loskill P (2021) Integration of electrospun membranes into low-absorption thermoplastic organ-on-chip. ACS Biomater Sci Eng 7:3006-3017.

Clements $\mathrm{M}$ and Thomas $\mathrm{N}$ (2014) High-throughput multi-parameter profiling of electrophysiological drug effects in human embryonic stem cell derived cardiomyocytes using multi-electrode arrays. Toxicol Sci 140:445-461.

Combes AN, Zappia L, Er PX, Oshlack A, and Little MH (2019) Single-cell analysis reveals congruence between kidney organoids and human fetal kidney. Genome Med 11:3.

Cook D, Brown D, Alexander R, March R, Morgan P, Satterthwaite G, and Pangalos MN (2014) Lessons learned from the fate of AstraZeneca's drug pipeline: a five-dimensional framework. Nat Rev Drug Discov 13:419-431.

Cordero-Herrera I, Kozyra M, Zhuge Z, McCann Haworth S, Moretti C, Peleli M, Caldeira-Dias M, Jahandideh A, and Huirong H, Cruz JdC, Kleschyov AL, Montenegro MF, Ingelman-Sundberg $\mathrm{M}$, Weitzberg $\mathrm{E}$, Lundberg JO and Carlström M (2019) AMP-activated protein kinase activation and NADPH oxidase inhibition by inorganic nitrate and nitrite prevent liver steatosis. Proceedings of the National Academy of Sciences of the United States of America 116:217-226.

Costello CM, Hongpeng J, Shaffiey S, Yu J, Jain NK, Hackam D, and March JC (2014) Synthetic small intestinal scaffolds for improved studies of intestina differentiation. Biotechnol Bioeng 111:1222-1232.

Costello CM, Phillipsen MB, Hartmanis LM, Kwasnica MA, Chen V, Hackam D, Chang MW, Bentley WE, and March JC (2017) Microscale bioreactors for in situ characterization of GI epithelial cell physiology. Sci Rep 7:12515.

Cruz-Acuña R, Quirós M, Farkas AE, Dedhia PH, Huang S, Siuda D, GarcíaHernández V, Miller AJ, Spence JR, Nusrat A et al. (2017) Synthetic hydrogels for human intestinal organoid generation and colonic wound repair. Nat Cell Biol 19:1326-1335.

Czerniecki SM, Cruz NM, Harder JL, Menon R, Annis J, Otto EA, Gulieva RE, Islas LV, Kim YK, Tran LM et al. (2018) High-throughput screening enhances kidney organoid differentiation from human pluripotent stem cells and enables automated multidimensional phenotyping. Cell Stem Cell 22:929-940.e4.

d'Aldebert E, Quaranta M, Sébert M, Bonnet D, Kirzin S, Portier G, Duffas J-P, Chabot S, Lluel P, Allart S et al. (2020) Characterization of human colon organoids from inflammatory bowel disease patients. Front Cell Dev Biol 8:363.

Da-Silva F, Boulenc X, Vermet H, Compigne P, Gerbal-Chaloin S, DaujatChavanieu M, Klieber S, and Poulin P (2018) Improving prediction of metabolic clearance using quantitative extrapolation of results obtained from human hepatic micropatterned cocultures model and by considering the impact of albumin binding. J Pharm Sci 107:1957-1972.

Dalvie D, Obach RS, Kang P, Prakash C, Loi C-M, Hurst S, Nedderman A, Goulet L, Smith E, Bu H-Z et al. (2009) Assessment of three human in vitro systems in the generation of major human excretory and circulating metabolites. Chem Res Toxicol 22:357-368.

Dang J, Tiwari SK, Lichinchi G, Qin Y, Patil VS, Eroshkin AM, and Rana TM (2016) Zika virus depletes neural progenitors in human cerebral organoids through activation of the innate immune receptor TLR3. Cell Stem Cell 19:258-265.

Danker T and Möller C (2014) Early identification of hERG liability in drug discovery programs by automated patch clamp. Front Pharmacol 5:203.

Darabi R, Arpke RW, Irion S, Dimos JT, Grskovic M, Kyba M, and Perlingeiro RCR (2012) Human ES- and iPS-derived myogenic progenitors restore DYSTROPHIN and improve contractility upon transplantation in dystrophic mice. Cell Stem Cell 10:610-619.

Davenport Huyer L, Bannerman AD, Wang Y, Savoji H, Knee-Walden EJ, Brissenden A, Yee B, Shoaib M, Bobicki E, Amsden BG et al. (2019) One-pot synthesis of unsaturated polyester bioelastomer with controllable materia curing for microscale designs. Adv Healthc Mater 8:e1900245.

Davidson MD, Ballinger KR, and Khetani SR (2016) Long-term exposure to abnormal glucose levels alters drug metabolism pathways and insulin sensitivity in primary human hepatocytes. Sci Rep 6:28178. 
Davidson MD, Kukla DA, and Khetani SR (2017) Microengineered cultures containing human hepatic stellate cells and hepatocytes for drug development. Integr Biol 9:662-677.

Davis BNJ, Santoso JW, Walker MJ, Cheng CS, Koves TR, Kraus WE, and Truskey GA (2017) Human, tissue-engineered, skeletal muscle myobundles to measure oxygen uptake and assess mitochondrial toxicity. Tissue Eng Part C Methods 23:189-199.

de Abajo FJ, Montero D, Madurga M, and García Rodríguez LA (2004) Acute and clinically relevant drug-induced liver injury: a population based case-control study. Br J Clin Pharmacol 58:71-80.

De Bruyn T, Chatterjee S, Fattah S, Keemink J, Nicolaı J, Augustijns P, and Annaert P (2013) Sandwich-cultured hepatocytes: utility for in vitro exploration of hepatobiliary drug disposition and drug-induced hepatotoxicity. Expert Opin Drug Metab Toxicol 9:589-616.

de Korte T, Katili PA, Mohd Yusof NAN, van Meer BJ, Saleem U, Burton FL, Smith GL, Clements P, Mummery CL, Eschenhagen T et al. (2020) Unlocking personalized biomedicine and drug discovery with human induced pluripotent stem cell-derived cardiomyocytes: fit for purpose or forever elusive? Annu Rev Pharmacol Toxicol 60:529-551.

Dervisevic E, Voelcker NH, Risbridger G, Tuck KL, and Cadarso VJ (2020) Highaspect-ratio SU-8-based optofluidic device for ammonia detection in cell culture media. ACS Sens 5:2523-2529.

Dimos JT, Rodolfa KT, Niakan KK, Weisenthal LM, Mitsumoto H, Chung W, Croft GF, Saphier G, Leibel R, Goland R et al. (2008) Induced pluripotent stem cells generated from patients with ALS can be differentiated into motor neurons. Science 321:1218-1221.

Dixon TA, Cohen E, Cairns DM, Rodriguez M, Mathews J, Jose RR, and Kaplan DL (2018) Bioinspired three-dimensional human neuromuscular junction development in suspended hydrogel arrays. Tissue Eng Part C Methods 24: 346-359.

Docci L, Klammers F, Ekiciler A, Molitor B, Umehara K, Walter I, Krähenbühl S, Parrott N, and Fowler S (2020) In vitro to in vivo extrapolation of metabolic clearance for UGT substrates using short-term suspension and long-term cocultured human hepatocytes. AAPS J 22:131.

Docci L, Parrott N, Krähenbühl S, and Fowler S (2019) Application of new cellular and microphysiological systems to drug metabolism optimization and their positioning respective to in silico tools. SLAS Discov 24:523-536.

Domansky K, Inman W, Serdy J, Dash A, Lim MHM, and Griffith LG (2010) Perfused multiwell plate for 3D liver tissue engineering. Lab Chip 10:51-58.

Douvaras P, Wang J, Zimmer M, Hanchuk S, O'Bara MA, Sadiq S, Sim FJ, Goldman J, and Fossati V (2014) Efficient generation of myelinating oligodendrocytes from primary progressive multiple sclerosis patients by induced pluripotent stem cells. Stem Cell Reports 3:250-259.

Drenth JPH and Schattenberg JM (2020) The nonalcoholic steatohepatitis (NASH) drug development graveyard: established hurdles and planning for future success. Expert Opin Investig Drugs 29:1365-1375.

Du C, Feng Y, Qiu D, Xu Y, Pang M, Cai N, Xiang AP, and Zhang Q (2018) Highly efficient and expedited hepatic differentiation from human pluripotent stem cells by pure small-molecule cocktails. Stem Cell Res Ther 9:58.

Du Y, Li N, Yang H, Luo C, Gong Y, Tong C, Gao Y, Lü S, and Long M (2017) Mimicking liver sinusoidal structures and functions using a 3D-configured microfluidic chip. Lab Chip 17:782-794.

Du Y, Wang J, Jia J, Song N, Xiang C, Xu J, Hou Z, Su X, Liu B, Jiang T et al. (2014) Human hepatocytes with drug metabolic function induced from fibroblasts by lineage reprogramming. Cell Stem Cell 14:394-403.

Dunn JC, Yarmush ML, Koebe HG, and Tompkins RG (1989) Hepatocyte function and extracellular matrix geometry: long-term culture in a sandwich configuration. FASEB J 3:174-177.

Dykens JA and Will Y (2007) The significance of mitochondrial toxicity testing in drug development. Drug Discov Today 12:777-785.

Eddings MA and Gale BK (2006) A PDMS-based gas permeation pump for on-chip fluid handling in microfluidic devices. J Micromech Microeng 16:2396.

Ehrlich A, Tsytkin-Kirschenzweig S, Ioannidis K, Ayyash M, Riu A, Note R, Ouedraogo G, Vanfleteren J, Cohen M, and Nahmias Y (2018) Microphysiological flux balance platform unravels the dynamics of drug induced steatosis. Lab Chip 18:2510-2522.

Eiraku M, Watanabe K, Matsuo-Takasaki M, Kawada M, Yonemura S, Matsumura M, Wataya T, Nishiyama A, Muguruma K, and Sasai Y (2008) Self-organized formation of polarized cortical tissues from ESCs and its active manipulation by extrinsic signals. Cell Stem Cell 3:519-532.

Elkayam T, Amitay-Shaprut S, Dvir-Ginzberg M, Harel T, and Cohen S (2006) Enhancing the drug metabolism activities of $\mathrm{C} 3 \mathrm{~A}-\mathrm{a}$ human hepatocyte cell lineby tissue engineering within alginate scaffolds. Tissue Eng 12:1357-1368.

Elwi AN, Damaraju VL, Kuzma ML, Mowles DA, Baldwin SA, Young JD, Sawyer MB, and Cass CE (2009) Transepithelial fluxes of adenosine and $2^{\prime}$ deoxyadenosine across human renal proximal tubule cells: roles of nucleoside transporters hENT1, hENT2, and hCNT3. Am J Physiol Renal Physiol 296:F1439-F1451.

Englund G, Rorsman F, Rönnblom A, Karlbom U, Lazorova L, Gråsjö J, Kindmark A, and Artursson P (2006) Regional levels of drug transporters along the human intestinal tract: co-expression of $\mathrm{ABC}$ and SLC transporters and comparison with Caco-2 cells. Eur J Pharm Sci 29:269-277.

Esch MB, Sung JH, Yang J, Yu C, Yu J, March JC, and Shuler ML (2012) On chip porous polymer membranes for integration of gastrointestinal tract epithelium with microfluidic 'body-on-a-chip' devices. Biomed Microdevices 14:895-906.

Esch MB, Ueno H, Applegate DR, and Shuler ML (2016) Modular, pumpless bodyon-a-chip platform for the co-culture of GI tract epithelium and 3D primary liver tissue. Lab Chip 16:2719-2729.
Ettayebi K, Crawford SE, Murakami K, Broughman JR, Karandikar U, Tenge VR, Neill FH, Blutt SE, Zeng X-L, Qu L et al. (2016) Replication of human noroviruses in stem cell-derived human enteroids. Science 353:1387-1393.

Fabre K, Berridge B, Proctor WR, Ralston S, Will Y, Baran SW, Yoder G, and Van Vleet TR (2020) Introduction to a manuscript series on the characterization and use of microphysiological systems (MPS) in pharmaceutical safety and ADME applications. Lab Chip 20:1049-1057.

Faulkner-Jones A, Fyfe C, Cornelissen D-J, Gardner J, King J, Courtney A, and Shu W (2015) Bioprinting of human pluripotent stem cells and their directed differentiation into hepatocyte-like cells for the generation of mini-livers in 3D. Biofabrication 7:044102.

Feaver RE, Cole BK, Lawson MJ, Hoang SA, Marukian S, Blackman BR, Figler RA, Sanyal AJ, Wamhoff BR, and Dash A (2016) Development of an in vitro human liver system for interrogating nonalcoholic steatohepatitis. JCI Insight 1:e90954

Fedecostante M, Westphal KGC, Buono MF, Sanchez Romero N, Wilmer MJ, Kerkering J, Baptista PM, Hoenderop JG, and Masereeuw R (2018) Recellularized native kidney scaffolds as a novel tool in nephrotoxicity screening. Drug Metab Dispos 46:1338-1350.

Ferri N, Siegl P, Corsini A, Herrmann J, Lerman A, and Benghozi R (2013) Drug attrition during pre-clinical and clinical development: understanding and managing drug-induced cardiotoxicity. Pharmacol Ther 138:470-484.

Fey SJ and Wrzesinski K (2012) Determination of drug toxicity using 3D spheroids constructed from an immortal human hepatocyte cell line. Toxicol Sci 127:403-411.

Finkbeiner SR, Hill DR, Altheim CH, Dedhia PH, Taylor MJ, Tsai Y-H, Chin AM, Mahe MM, Watson CL, Freeman JJ et al. (2015) Transcriptome-wide analysis reveals hallmarks of human intestine development and maturation in vitro and in vivo. Stem Cell Reports 4:1140-1155.

Foster AJ, Chouhan B, Regan SL, Rollison H, Amberntsson S, Andersson LC, Srivastava A, Darnell M, Cairns J, Lazic SE et al. (2019) Integrated in vitro models for hepatic safety and metabolism: evaluation of a human liver-chip and liver spheroid. Arch Toxicol 93:1021-1037.

Foster E, You J, Siltanen C, Patel D, Haque A, Anderson L, and Revzin A (2015) Heparin hydrogel sandwich cultures of primary hepatocytes. Eur Polym $J$ 72:726-735.

Fowler S, Chen WLK, Duignan DB, Gupta A, Hariparsad N, Kenny JR, Lai WG Liras J, Phillips JA, and Gan J (2020) Microphysiological systems for ADMErelated applications: current status and recommendations for system development and characterization. Lab Chip 20:446-467.

Frampton JP, Hynd MR, Shuler ML, and Shain W (2011) Fabrication and optimization of alginate hydrogel constructs for use in $3 \mathrm{D}$ neural cell culture. Biomed Mater 6:015002.

Freag MS, Namgung B, Reyna Fernandez ME, Gherardi E, Sengupta S, and Jang HL (2020) Human nonalcoholic steatohepatitis on a chip. Hepatol Commun 5:217-233.

Fu G-B, Huang W-J, Zeng M, Zhou X, Wu H-P, Liu C-C, Wu H, Weng J, Zhang HD, Cai Y-C et al. (2019) Expansion and differentiation of human hepatocytederived liver progenitor-like cells and their use for the study of hepatotropic pathogens. Cell Res 29:8-22.

Gabriel E, Wason A, Ramani A, Gooi LM, Keller P, Pozniakovsky A, Poser I, Noack F, Telugu NS, Calegari F et al. (2016) CPAP promotes timely cilium disassembly to maintain neural progenitor pool. EMBO J 35:803-819.

Gao D, Liu H, Lin J-M, Wang Y, and Jiang Y (2013) Characterization of drug permeability in Caco-2 monolayers by mass spectrometry on a membrane-based microfluidic device. Lab Chip 13:978-985.

Garcez PP, Loiola EC, Madeiro da Costa R, Higa LM, Trindade P, Delvecchio R, Nascimento JM, Brindeiro R, Tanuri A, and Rehen SK (2016) Zika virus impairs growth in human neurospheres and brain organoids. Science 352:816-818.

Garreta E, Prado P, Tarantino C, Oria R, Fanlo L, Martí E, Zalvidea D, Trepat X, Roca-Cusachs P, Gavaldà-Navarro A et al. (2019) Fine tuning the extracellular environment accelerates the derivation of kidney organoids from human pluripotent stem cells. Nat Mater 18:397-405.

Gaskell H, Sharma P, Colley HE, Murdoch C, Williams DP, and Webb SD (2016) Characterization of a functional C3A liver spheroid model. Toxicol Res (Camb) 5:1053-1065.

Gerets HHJ, Tilmant K, Gerin B, Chanteux H, Depelchin BO, Dhalluin S, and Atienzar FA (2012) Characterization of primary human hepatocytes, HepG2 cells, and HepaRG cells at the mRNA level and CYP activity in response to inducers and their predictivity for the detection of human hepatotoxins. Cell Biol Toxicol 28:69-87.

Gijzen L, Marescotti D, Raineri E, Nicolas A, Lanz HL, Guerrera D, van Vught R, Joore J, Vulto P, Peitsch MC et al. (2020) An intestine-on-a-chip model of plugand-play modularity to study inflammatory processes. SLAS Technol 25:585-597.

Gjorevski N, Avignon B, Gérard R, Cabon L, Roth AB, Bscheider M, and Moisan A (2020) Neutrophilic infiltration in organ-on-a-chip model of tissue inflammation. Lab Chip 20:3365-3374.

Gjorevski N, Sachs N, Manfrin A, Giger S, Bragina ME, Ordóñez-Morán P, Clevers $\mathrm{H}$, and Lutolf MP (2016) Designer matrices for intestinal stem cell and organoid culture. Nature 539:560-564.

Glezos N, Misiakos K, Kakabakos S, Petrou P, and Terzoudi G (2002) Electron beam patterning of biomolecules. Biosens Bioelectron 17:279-282.

Godoy P, Hengstler JG, Ilkavets I, Meyer C, Bachmann A, Müller A, Tuschl G, Mueller SO, and Dooley S (2009) Extracellular matrix modulates sensitivity of hepatocytes to fibroblastoid dedifferentiation and transforming growth factor $\beta$-induced apoptosis. Hepatology 49:2031-2043.

Goldfracht I, Efraim Y, Shinnawi R, Kovalev E, Huber I, Gepstein A, Arbel G, Shaheen N, Tiburcy M, Zimmermann WH et al. (2019) Engineered heart tissue models from hiPSC-derived cardiomyocytes and cardiac ECM for disease modeling and drug testing applications. Acta Biomater 92:145-159. 
Goldring C, Antoine DJ, Bonner F, Crozier J, Denning C, Fontana RJ, Hanley NA, Hay DC, Ingelman-Sundberg M, Juhila S et al. (2017) Stem cell-derived models to improve mechanistic understanding and prediction of human drug-induced liver injury. Hepatology 65:710-721.

Gómez-Lechón MJ, Tolosa L, Conde I, and Donato MT (2014) Competency of different cell models to predict human hepatotoxic drugs. Expert Opin Drug Metab Toxicol 10:1553-1568.

Gori M, Simonelli MC, Giannitelli SM, Businaro L, Trombetta M, and Rainer A (2016) Investigating nonalcoholic fatty liver disease in a liver-on-a-chip microfluidic device. PLoS One 11:e0159729.

Gracz AD, Williamson IA, Roche KC, Johnston MJ, Wang F, Wang Y, Attayek PJ, Balowski J, Liu XF, Laurenza RJ et al. (2015) A high-throughput platform for stem cell niche co-cultures and downstream gene expression analysis. Nat Cell Biol 17:340-349.

Grassart A, Malardé V, Gobaa S, Sartori-Rupp A, Kerns J, Karalis K, Marteyn B, Sansonetti P, and Sauvonnet N (2019) Bioengineered human organ-on-chip reveals intestinal microenvironment and mechanical forces impacting Shigella infection. Cell Host Microbe 26:435-444.e4.

Grosberg A, Alford PW, McCain ML, and Parker KK (2011) Ensembles of engineered cardiac tissues for physiological and pharmacological study: heart on a chip. Lab Chip 11:4165-4173.

Gudapati H, Dey M, and Ozbolat I (2016) A comprehensive review on droplet-based bioprinting: Past, present and future. Biomaterials 102:20-42.

Guillotin B, Catros S, and Guillemot F (2013) Laser assisted bio-printing (LAB) of cells and bio-materials based on laser induced forward transfer (LIFT), in Laser Technology in Biomimetics. Biological and Medical Physics, Biomedical Engineering (Schmidt V and Belegratis M, eds Springer, Berlin, Heidelberg., https://doi.org/10.1007/978-3-642-41341-4_8.

Guillotin B, Souquet A, Catros S, Duocastella M, Pippenger B, Bellance S, Bareille R, Rémy M, Bordenave L, Amédée J et al. (2010) Laser assisted bioprinting of engineered tissue with high cell density and microscale organization. Biomaterials 31:7250-7256.

Gungor-Ozkerim PS, Inci I, Zhang YS, Khademhosseini A, and Dokmeci MR (2018) Bioinks for 3D bioprinting: an overview. Biomater Sci 6:915-946.

Gunness P, Mueller D, Shevchenko V, Heinzle E, Ingelman-Sundberg M, and Noor F (2013) 3D organotypic cultures of human HepaRG cells: a tool for in vitro toxicity studies. Toxicol Sci 133:67-78.

Guo LJ (2007) Nanoimprint lithography: methods and material requirements. $A d v$ Mater 19:495-513.

Gupta R, Schrooders Y, Hauser D, van Herwijnen M, Albrecht W, Ter Braak B, Brecklinghaus T, Castell JV, Elenschneider L, Escher S et al. (2021) Comparing in vitro human liver models to in vivo human liver using RNA-Seq. Arch Toxicol 95:573-589.

Gural N, Mancio-Silva L, Miller AB, Galstian A, Butty VL, Levine SS, Patrapuvich R, Desai SP, Mikolajczak SA, Kappe SHI et al. (2018) In vitro culture, drug sensitivity, and transcriptome of plasmodium vivax hypnozoites. Cell Host Microbe 23:395-406.e4

Hackam DG and Redelmeier DA (2006) Translation of research evidence from animals to humans. JAMA 296:1731-1732.

Haenseler W, Sansom SN, Buchrieser J, Newey SE, Moore CS, Nicholls FJ, Chintawar S, Schnell C, Antel JP, Allen ND et al. (2017) A highly efficient human pluripotent stem cell microglia model displays a neuronal-co-culturespecific expression profile and inflammatory response. Stem Cell Reports 8:1727-1742

Hagmann H and Brinkkoetter PT (2018) Experimental models to study podocyte biology: stock-taking the toolbox of glomerular research. Front Pediatr 6:193.

Hale LJ, Howden SE, Phipson B, Lonsdale A, Er PX, Ghobrial I, Hosawi S, Wilson S, Lawlor KT, Khan S et al. (2018) 3D organoid-derived human glomeruli for personalised podocyte disease modelling and drug screening. Nat Commun 9:5167.

Hannan NRF, Segeritz C-P, Touboul T, and Vallier L (2013) Production of hepatocyte-like cells from human pluripotent stem cells. Nat Protoc 8:430-437.

Hansen A, Eder A, Bönstrup M, Flato M, Mewe M, Schaaf S, Aksehirlioglu B, Schwoerer AP, Uebeler J, and Eschenhagen T (2010) Development of a drug screening platform based on engineered heart tissue. Circ Res 107:35-44.

Harris K (2015) A human induced pluripotent stem cell-derived cardiomyocyte (hiPSC-CM) multielectrode array assay for preclinical cardiac electrophysiology safety screening. Current Protocols in Pharmacology 71:11.18.11-11.18.15.

Hart SN, Li Y, Nakamoto K, Subileau EA, Steen D, and Zhong XB (2010) A comparison of whole genome gene expression profiles of HepaRG cells and HepG2 cells to primary human hepatocytes and human liver tissues. Drug Metab Dispos 38:988-994.

Hay M, Thomas DW, Craighead JL, Economides C, and Rosenthal J (2014) Clinical development success rates for investigational drugs. Nat Biotechnol 32:40-51.

Hayeshi R, Hilgendorf C, Artursson P, Augustijns P, Brodin B, Dehertogh P, Fisher K, Fossati L, Hovenkamp E, Korjamo T et al. (2008) Comparison of drug transporter gene expression and functionality in Caco-2 cells from 10 different laboratories. Eur J Pharm Sci 35:383-396.

Heckele M and Schomburg WK (2003) Review on micro molding of thermoplastic polymers. J Micromech Microeng 14:R1-R14.

Heinrich MA, Liu W, Jimenez A, Yang J, Akpek A, Liu X, Pi Q, Mu X, Hu N, Schiffelers RM et al. (2019) 3D bioprinting: from benches to translational applications. Small 15:e1805510.

Hendriks DFG, Fredriksson Puigvert L, Messner S, Mortiz W, and IngelmanSundberg M (2016) Hepatic 3D spheroid models for the detection and study of compounds with cholestatic liability. Sci Rep 6:35434.

Hendriks DFG, Hurrell T, Riede J, van der Horst M, Tuovinen S, and IngelmanSundberg M (2019) Mechanisms of chronic fialuridine hepatotoxicity as revealed in primary human hepatocyte spheroids. Toxicol Sci 171:385-395.
Hendriks DFG, Vorrink SU, Smutny T, Sim SC, Nordling A, Ullah S, Kumondai M, Jones BC, Johansson I, Andersson TB et al. (2020) Clinically relevant cytochrome P450 3A4 induction mechanisms and drug screening in threedimensional spheroid cultures of primary human hepatocytes. Clin Pharmacol Ther 108:844-855.

Heo I, Dutta D, Schaefer DA, Iakobachvili N, Artegiani B, Sachs N, Boonekamp KE, Bowden G, Hendrickx APA, Willems RJL et al. (2018) Modelling Cryptosporidium infection in human small intestinal and lung organoids. Nat Microbiol 3:814-823.

Herland A, van der Meer AD, FitzGerald EA, Park T-E, Sleeboom JJF, and Ingber DE (2016) Distinct contributions of astrocytes and pericytes to meuroinflammation identified in a 3D human blood-brain barrier on a chip. PLoS One 11:e0150360.

Heslop JA, Rowe C, Walsh J, Sison-Young R, Jenkins R, Kamalian L, Kia R, Hay D, Jones RP, Malik HZ et al. (2017) Mechanistic evaluation of primary human hepatocyte culture using global proteomic analysis reveals a selective dedifferentiation profile. Arch Toxicol 91:439-452.

Hilgendorf C, Spahn-Langguth H, Regårdh CG, Lipka E, Amidon GL, and Langguth P (2000) Caco-2 versus Caco-2/HT29-MTX co-cultured cell lines: permeabilities via diffusion, inside- and outside-directed carrier-mediated transport. $J$ Pharm Sci 89:63-75.

Hiller T, Berg J, Elomaa L, Röhrs V, Ullah I, Schaar K, Dietrich A-C, Al-Zeer MA, Kurtz A, Hocke AC et al. (2018) Generation of a 3D liver model comprising human extracellular matrix in an alginate/gelatin-based bioink by extrusion bioprinting for infection and transduction studies. Int J Mol Sci 19:3129.

Hinds S, Bian W, Dennis RG, and Bursac N (2011) The role of extracellular matrix composition in structure and function of bioengineered skeletal muscle. Biomaterials 32:3575-3583.

Hinson JT, Chopra A, Nafissi N, Polacheck WJ, Benson CC, Swist S, Gorham J, Yang L, Schafer S, Sheng CC et al. (2015) Heart disease. Titin mutations in iPS cells define sarcomere insufficiency as a cause of dilated cardiomyopathy. Science 349:982-986.

Hiratsuka K, Monkawa T, Akiyama T, Nakatake Y, Oda M, Goparaju SK, Kimura H, Chikazawa-Nohtomi N, Sato S, Ishiguro K et al. (2019) Induction of human pluripotent stem cells into kidney tissues by synthetic mRNAs encoding transcription factors. Sci Rep 9:913.

Hirt MN, Boeddinghaus J, Mitchell A, Schaaf S, Börnchen C, Müller C, Schulz H Hubner N, Stenzig J, Stoehr A et al. (2014) Functional improvement and maturation of rat and human engineered heart tissue by chronic electrical stimulation. J Mol Cell Cardiol 74:151-161.

Hirt MN, Sörensen NA, Bartholdt LM, Boeddinghaus J, Schaaf S, Eder A, Vollert I, Stöhr A, Schulze T, Witten A et al. (2012) Increased afterload induces pathological cardiac hypertrophy: a new in vitro model. Basic Res Cardiol 107:307.

Holly MK and Smith JG (2018) Adenovirus infection of human enteroids reveals interferon sensitivity and preferential infection of goblet cells. $J$ Virol 92 e00250-e18.

Holmberg FE, Seidelin JB, Yin X, Mead BE, Tong Z, Li Y, Karp JM, and Nielsen $\mathrm{OH}$ (2017) Culturing human intestinal stem cells for regenerative applications in the treatment of inflammatory bowel disease. EMBO Mol Med 9:558-570.

Homan KA, Gupta N, Kroll KT, Kolesky DB, Skylar-Scott M, Miyoshi T, Mau D Valerius MT, Ferrante T, Bonventre JV et al. (2019) Flow-enhanced vascularization and maturation of kidney organoids in vitro. Nat Methods 16:255-262.

Homan KA, Kolesky DB, Skylar-Scott MA, Herrmann J, Obuobi H, Moisan A, and Lewis JA (2016) Bioprinting of 3D convoluted renal proximal tubules on perfusable chips. Sci Rep 6:34845

Hu B-Y, Du Z-W, and Zhang S-C (2009) Differentiation of human oligodendrocytes from pluripotent stem cells. Nat Protoc 4:1614-1622.

Huang JX, Kaeslin G, Ranall MV, Blaskovich MA, Becker B, Butler MS, Little MH, Lash LH, and Cooper MA (2015) Evaluation of biomarkers for in vitro prediction of drug-induced nephrotoxicity: comparison of HK-2, immortalized human proximal tubule epithelial, and primary cultures of human proximal tubular cells. Pharmacol Res Perspect 3:e00148.

Hubert CG, Rivera M, Spangler LC, Wu Q, Mack SC, Prager BC, Couce M, McLendon RE, Sloan AE, and Rich JN (2016) A three-dimensional organoid culture system derived from human glioblastomas recapitulates the hypoxic gradients and cancer stem cell heterogeneity of tumors found in vivo. Cancer Res 76:2465-2477.

Huebsch N, Loskill P, Deveshwar N, Spencer CI, Judge LM, Mandegar MA, Fox CB, Mohamed TMA, Ma Z, Mathur A et al. (2016) Miniaturized iPS-cell-derived cardiac muscles for physiologically relevant drug response analyses. Sci Rep 6:24726

Hughes B (2008) Industry concern over EU hepatotoxicity guidance. Nat Rev Drug Discov 7:719.

Hultman Ia, Vedin C, Abrahamsson A, Winiwarter S, and Darnell M (2016) Use of $\mathrm{H} \mu \mathrm{REL}$ human coculture system for prediction of intrinsic clearance and metabolite formation for slowly metabolized compounds. Mol Pharm 13:2796-2807.

Hundertmark J and Tacke F (2020) How effective are nonalcoholic fatty liver disease models for drug discovery? Expert Opin Drug Discov 15:1237-1240.

Hurrell T, Kastrinou-Lampou V, Fardellas A, Hendriks DFG, Nordling A Johansson I, Baze A, Parmentier C, Richert L, and Ingelman-Sundberg M (2020) Human liver spheroids as a model to study aetiology and treatment of hepatic fibrosis. Cells 9:964.

Huszthy PC, Daphu I, Niclou SP, Stieber D, Nigro JM, Sakariassen Pł, Miletic H Thorsen F, and Bjerkvig R (2012) In vivo models of primary brain tumors: pitfalls and perspectives. Neuro-oncol 14:979-993.

Iefremova V, Manikakis G, Krefft O, Jabali A, Weynans K, Wilkens R, Marsoner F, Brändl B, Müller F-J, Koch P et al. (2017) An organoid-based model of cortical development identifies non-cell-autonomous defects in Wnt signaling contributing to Miller-Dieker syndrome. Cell Rep 19:50-59. 
Ildefonso M, Candoni N, and Veesler S (2012) A cheap, easy microfluidic crystallization device ensuring universal solvent compatibility. Org Process Res Dev 16:556-560

Imura Y, Asano Y, Sato K, and Yoshimura E (2009) A microfluidic system to evaluate intestinal absorption. Anal Sci 25:1403-1407.

Imura Y, Sato K, and Yoshimura E (2010) Micro total bioassay system for ingested substances: assessment of intestinal absorption, hepatic metabolism, and bioactivity. Anal Chem 82:9983-9988.

Imura Y, Yoshimura E, and Sato K (2012) Micro total bioassay system for oral drugs: evaluation of gastrointestinal degradation, intestinal absorption, hepatic metabolism, and bioactivity. Anal Sci 28:197-199.

In J, Foulke-Abel J, Zachos NC, Hansen A-M, Kaper JB, Bernstein HD, Halushka M, Blutt S, Estes MK, Donowitz M et al. (2016) Enterohemorrhagic Escherichia coli reduce mucus and intermicrovillar bridges in human stem cell-derived colonoids. Cell Mol Gastroenterol Hepatol 2:48-62.e3.

Ingelman-Sundberg M and Lauschke VM (2018) Human liver spheroids in chemically defined conditions for studies of gene-drug, drug-drug and diseasedrug interactions. Pharmacogenomics 19:1133-1138.

Ingelman-Sundberg $M$ and Lauschke VM (2021) 3D human liver spheroids for translational pharmacology and toxicology. Basic \& Clinical Pharmacology \& Toxicology. doi: https://doi.org/10.1111/bcpt.13587. Online ahead of print.

Itzhaki I, Maizels L, Huber I, Zwi-Dantsis L, Caspi O, Winterstern A, Feldman O, Gepstein A, Arbel G, Hammerman H et al. (2011) Modelling the long QT syndrome with induced pluripotent stem cells. Nature 471:225-229.

Jabaji Z, Sears CM, Brinkley GJ, Lei NY, Joshi VS, Wang J, Lewis M, Stelzner M, Martín MG, and Dunn JCY (2013) Use of collagen gel as an alternative extracellular matrix for the in vitro and in vivo growth of murine small intestinal epithelium. Tissue Eng Part C Methods 19:961-969.

Jacob F, Pather SR, Huang W-K, Zhang F, Wong SZH, Zhou H, Cubitt B, Fan W, Chen CZ, Xu M et al. (2020) Human pluripotent stem cell-derived neural cells and brain organoids reveal SARS-CoV-2 neurotropism predominates in choroid plexus epithelium. Cell Stem Cell 27:937-950.e9.

Jalal S, Dastidar S, and Tedesco FS (2021) Advanced models of human skeletal muscle differentiation, development and disease: three-dimensional cultures, organoids and beyond. Curr Opin Cell Biol 73:92-104

Jalili-Firoozinezhad S, Gazzaniga FS, Calamari EL, Camacho DM, Fadel CW, Bein A, Swenor B, Nestor B, Cronce MJ, Tovaglieri A et al. (2019) A complex human gut microbiome cultured in an anaerobic intestine-on-a-chip. Nat Biomed Eng 3:520-531.

Janero DR (2014) The future of drug discovery: enabling technologies for enhancing lead characterization and profiling therapeutic potential. Expert Opin Drug Discov 9:847-858.

Jang K-J, Mehr AP, Hamilton GA, McPartlin LA, Chung S, Suh KY, and Ingber DE (2013) Human kidney proximal tubule-on-a-chip for drug transport and nephrotoxicity assessment. Integr Biol 5:1119-1129.

Jang K-J, Otieno MA, Ronxhi J, Lim H-K, Ewart L, Kodella KR, Petropolis DB, Kulkarni G, Rubins JE, Conegliano D et al. (2019a) Reproducing human and cross-species drug toxicities using a liver-chip. Sci Transl Med 11:eaax5516.

Jang K-J and Suh KY (2010) A multi-layer microfluidic device for efficient culture and analysis of renal tubular cells. Lab Chip 10:36-42.

Jang M, Kleber A, Ruckelshausen T, Betzholz R, and Manz A (2019b) Differentiation of the human liver progenitor cell line (HepaRG) on a microfluidic-based biochip. $J$ Tissue Eng Regen Med 13:482-494.

Jang M, Neuzil P, Volk T, Manz A, and Kleber A (2015) On-chip three-dimensional cell culture in phaseguides improves hepatocyte functions in vitro. Biomicrofluidics 9:034113

Jansen J, De Napoli IE, Fedecostante M, Schophuizen CMS, Chevtchik NV, Wilmer MJ, van Asbeck AH, Croes HJ, Pertijs JC, Wetzels JFM et al. (2015) Human proximal tubule epithelial cells cultured on hollow fibers: living membranes that actively transport organic cations. Sci Rep 5:16702

Jeon J-W, Lee SH, Kim D, and Sung JH (2021) In vitro hepatic steatosis model based on gut-liver-on-a-chip. Biotechnology Progress DOI: https://doi.org/10.1002/ btpr.3121 [published ahead of print].

Jiang J, Zhan J, Yue W, Yang M, Yi C, and Li C-W (2015) A single low-cost microfabrication approach for polymethylmethacrylate, polystyrene, polycarbonate and polysulfone based microdevices. RSC Advances 5:36036-36043.

Jo J, Xiao Y, Sun AX, Cukuroglu E, Tran H-D, Göke J, Tan ZY, Saw TY, Tan C-P, Lokman $\mathrm{H}$ et al. (2016) Midbrain-like organoids from human pluripotent stem cells contain functional dopaminergic and neuromelanin-producing neurons. Cell Stem Cell 19:248-257.

Juhas M and Bursac N (2014) Roles of adherent myogenic cells and dynamic culture in engineered muscle function and maintenance of satellite cells. Biomaterials 35:9438-9446.

Kadoshima T, Sakaguchi H, Nakano T, Soen M, Ando S, Eiraku M, and Sasai Y (2013) Self-organization of axial polarity, inside-out layer pattern, and speciesspecific progenitor dynamics in human ES cell-derived neocortex. Proc Natl Acad Sci USA 110:20284-20289.

Kamel A, Bowlin S, Hosea N, Arkilo D, and Laurenza A (2021) In vitro metabolism of slowly cleared $\mathrm{G}$ protein-coupled receptor 139 agonist TAK-041 using rat, dog, monkey, and human hepatocyte models (HepatoPac): correlation with in vivo metabolism. Drug Metab Dispos 49:121-132.

Kandasamy K, Chuah JKC, Su R, Huang P, Eng KG, Xiong S, Li Y, Chia CS, Loo L-H, and Zink D (2015) Prediction of drug-induced nephrotoxicity and injury mechanisms with human induced pluripotent stem cell-derived cells and machine learning methods. Sci Rep 5:12337.

Kanebratt KP, Janefeldt A, Vilén L, Vildhede A, Samuelsson K, Milton L, Björkbom A, Persson M, Leandersson C, Andersson TB et al. (2021) Primary human hepatocyte spheroid model as a $3 \mathrm{D}$ in vitro platform for metabolism studies. $J$ Pharm Sci 110:422-431.
Kang D, Ahn G, Kim D, Kang H-W, Yun S, Yun W-S, Shim J-H, and Jin S (2018) Pre-set extrusion bioprinting for multiscale heterogeneous tissue structure fabrication. Biofabrication 10:035008.

Kang MS, Lee SH, Park WJ, Lee JE, Kim B, and Han D-W (2020) Advanced techniques for skeletal muscle tissue engineering and regeneration. Bioengineering (Basel) 7:99.

Kararli TT (1995) Comparison of the gastrointestinal anatomy, physiology, and biochemistry of humans and commonly used laboratory animals. Biopharm Drug Dispos 16:351-380.

Kasendra M, Tovaglieri A, Sontheimer-Phelps A, Jalili-Firoozinezhad S, Bein A Chalkiadaki A, Scholl W, Zhang C, Rickner H, Richmond CA et al. (2018) Development of a primary human small intestine-on-a-chip using biopsy-derived organoids. Sci Rep 8:2871.

Katayama R, Nagata S, Iida H, Yamagishi N, Yamashita T, and Furuhama K (2011) Possible role of cysteine-S-conjugate $\beta$-lyase in species differences in cisplatin nephrotoxicity. Food Chem Toxicol 49:2053-2059.

Kathuria A, Lopez-Lengowski K, Jagtap SS, McPhie D, Perlis RH, Cohen BM, and Karmacharya R (2020) Transcriptomic landscape and functional characterization of induced pluripotent stem cell-derived cerebral organoids in schizophrenia. JAMA Psychiatry 77:745-754.

Kato-Negishi M, Morimoto Y, Onoe H, and Takeuchi S (2013) Millimeter-sized neural building blocks for 3D heterogeneous neural network assembly. Adv Healthc Mater 2:1564-1570.

Katsuda H, Yamashita M, Katsura H, Yu J, Waki Y, Nagata N, Sai Y, and Miyamoto K (2010) Protecting cisplatin-induced nephrotoxicity with cimetidine does not affect antitumor activity. Biol Pharm Bull 33:1867-1871.

Katsuda T, Kawamata M, Inoue A, Yamaguchi T, Abe M, and Ochiya T (2020) Long-term maintenance of functional primary human hepatocytes using small molecules. FEBS Lett 594:114-125.

Kemas AM, Youhanna S, Zandi Shafagh R, and Lauschke VM (2021) Insulindependent glucose consumption dynamics in 3D primary human liver cultures measured by a sensitive and specific glucose sensor with nanoliter input volume. FASEB J 35:e21305.

Kern A, Bader A, Pichlmayr R, and Sewing KF (1997) Drug metabolism in hepatocyte sandwich cultures of rats and humans. Biochem Pharmacol 54:761-772.

Khetani SR and Bhatia SN (2008) Microscale culture of human liver cells for drug development. Nat Biotechnol 26:120-126.

Khetani SR, Kanchagar C, Ukairo O, Krzyzewski S, Moore A, Shi J, Aoyama S, Aleo M, and Will Y (2013) Use of micropatterned cocultures to detect compounds that cause drug-induced liver injury in humans. Toxicol Sci 132:107-117.

Kidambi S, Yarmush RS, Novik E, Chao P, Yarmush ML, and Nahmias Y (2009) Oxygen-mediated enhancement of primary hepatocyte metabolism, functional polarization, gene expression, and drug clearance. Proc Natl Acad Sci USA 106:15714-15719.

Kietzmann T (2017) Metabolic zonation of the liver: the oxygen gradient revisited. Redox Biol 11:622-630.

Kilpinen H, Goncalves A, Leha A, Afzal V, Alasoo K, Ashford S, Bala S, Bensaddek D, Casale FP, Culley OJ et al. (2017) Common genetic variation drives molecular heterogeneity in human iPSCs. Nature 546:370-375.

Kim D-S, Choi YW, Shanmugasundaram A, Jeong Y-J, Park J, Oyunbaatar N-E, Kim E-S, Choi M, and Lee D-W (2020a) Highly durable crack sensor integrated with silicone rubber cantilever for measuring cardiac contractility. Nat Commun 11:535.

Kim H, Park HJ, Choi H, Chang Y, Park H, Shin J, Kim J, Lengner CJ, Lee YK, and Kim J (2019) Modeling G2019S-LRRK2 sporadic Parkinson's disease in 3D midbrain Organoids. Stem Cell Reports 12:518-531.

Kim HJ, Huh D, Hamilton G, and Ingber DE (2012) Human gut-on-a-chip inhabited by microbial flora that experiences intestinal peristalsis-like motions and flow. Lab Chip 12:2165-2174.

Kim HJ and Ingber DE (2013) Gut-on-a-chip microenvironment induces human intestinal cells to undergo villus differentiation. Integr Biol 5:1130-1140.

Kim HJ, Li H, Collins JJ, and Ingber DE (2016a) Contributions of microbiome and mechanical deformation to intestinal bacterial overgrowth and inflammation in a human gut-on-a-chip. Proc Natl Acad Sci USA 113:E7-E15.

Kim JH, Seol Y-J, Ko IK, Kang H-W, Lee YK, Yoo JJ, Atala A, and Lee SJ (2018) 3D bioprinted human skeletal muscle constructs for muscle function restoration. Sci Rep 8:12307.

Kim JW, Nam SA, Seo E, Lee J-Y, Kim D, Ju JH, Lim SW, Kim HL, Kim HW, Yang CW, Kim J, Kim DS and Kim YK (2020b) Human kidney organoids model the tacrolimus nephrotoxicity and elucidate the role of autophagy. The Korean Journal of Internal Medicine DOI: https://doi.org/10.3904/kjim.2020.323 [published ahead of print].

Kim MH, Kumar SK, Shirahama H, Seo J, Lee JH, and Cho N-J (2016b) Phenotypic regulation of liver cells in a biofunctionalized three-dimensional hydrogel platform. Integr Biol 8:156-166.

Kim SH, Chi M, Yi B, Kim SH, Oh S, Kim Y, Park S, and Sung JH (2014) Threedimensional intestinal villi epithelium enhances protection of human intestinal cells from bacterial infection by inducing mucin expression. Integr Biol 6:1122-1131.

Kimoto E, Walsky R, Zhang H, Bi YA, Whalen KM, Yang YS, Linder C, Xiao Y, Iseki K Fenner KS et al (2012) Differential modulation of cytochrome P450 activity and the effect of 1-aminobenzotriazole on hepatic transport in sandwichcultured human hepatocytes. Drug Metab Dispos 40:407-411.

Kimura H, Ikeda T, Nakayama H, Sakai Y, and Fujii T (2015) An on-chip smal intestine-liver model for pharmacokinetic studies. J Lab Autom 20:265-273.

Kimura H, Yamamoto T, Sakai H, Sakai Y, and Fujii T (2008) An integrated microfluidic system for long-term perfusion culture and on-line monitoring of intestinal tissue models. Lab Chip 8:741-746. 
King SM, Higgins JW, Nino CR, Smith TR, Paffenroth EH, Fairbairn CE, Docuyanan A, Shah VD, Chen AE, Presnell SC et al. (2017) 3D proximal tubule tissues recapitulate key aspects of renal physiology to enable nephrotoxicity testing. Front Physiol 8:123.

Klebe RJ (1988) Cytoscribing: a method for micropositioning cells and the construction of two- and three-dimensional synthetic tissues. Exp Cell Res 179:362-373.

Klein E, Hau A-C, Oudin A, Golebiewska A, and Niclou SP (2020) Glioblastoma organoids: pre-clinical applications and challenges in the context of immunotherapy. Front Oncol 10:604121.

Knauer MJ, Urquhart BL, Meyer zu Schwabedissen HE, Schwarz UI, Lemke CJ, Leake BF, Kim RB, and Tirona RG (2010) Human skeletal muscle drug transporters determine local exposure and toxicity of statins. Circ Res 106:297-306. Koide N, Shinji T, Tanabe T, Asano K, Kawaguchi M, Sakaguchi K, Koide Y, Mori M, and Tsuji T (1989) Continued high albumin production by multicellular spheroids of adult rat hepatocytes formed in the presence of liver-derived proteoglycans. Biochem Biophys Res Commun 161:385-391.

Koike M, Kurosawa H, and Amano Y (2005) A round-bottom 96-well polystyrene plate coated with 2-methacryloyloxyethyl phosphorylcholine as an effective tool for embryoid body formation. Cytotechnology 47:3-10.

Kojima K, Kaneko T, and Yasuda K (2006) Role of the community effect of cardiomyocyte in the entrainment and reestablishment of stable beating rhythms. Biochem Biophys Res Commun 351:209-215.

Kostrzewski T, Cornforth T, Snow SA, Ouro-Gnao L, Rowe C, Large EM, and Hughes DJ (2017) Three-dimensional perfused human in vitro model of nonalcoholic fatty liver disease. World J Gastroenterol 23:204-215.

Kostrzewski T, Maraver P, Ouro-Gnao L, Levi A, Snow S, Miedzik A, Rombouts K, and Hughes D (2019) A microphysiological system for studying nonalcoholic steatohepatitis. Hepatol Commun 4:77-91.

Kozuka K, He Y, Koo-McCoy S, Kumaraswamy P, Nie B, Shaw K, Chan P, Leadbetter M, He L, Lewis JG et al. (2017) Development and characterization of a human and mouse intestinal epithelial cell monolayer platform. Stem Cell Reports 9:1976-1990.

Kozyra M, Johansson I, Nordling Å, Ullah S, Lauschke VM, and IngelmanSundberg M (2018) Human hepatic 3D spheroids as a model for steatosis and insulin resistance. Sci Rep 8:14297.

Kratochwil NA, Meille C, Fowler S, Klammers F, Ekiciler A, Molitor B, Simon S, Walter I, McGinnis C, Walther J et al. (2017) Metabolic profiling of human longterm liver models and hepatic clearance predictions from in vitro data using nonlinear mixed-effects modeling. AAPS J 19:534-550.

Krencik R, Weick JP, Liu Y, Zhang Z-J, and Zhang S-C (2011) Specification of transplantable astroglial subtypes from human pluripotent stem cells. Nat Biotechnol 29:528-534.

Krug SM, Amasheh S, Richter JF, Milatz S, Günzel D, Westphal JK, Huber O, Schulzke JD, and Fromm M (2009) Tricellulin forms a barrier to macromolecules in tricellular tight junctions without affecting ion permeability. Mol Biol Cell 20:3713-3724.

Krüger J, Groß R, Conzelmann C, Müller JA, Koepke L, Sparrer KMJ, Weil T, Schütz D, Seufferlein T, Barth TFE et al. (2020) Drug inhibition of SARS-CoV-2 replication in human pluripotent stem cell-derived intestinal organoids. Cell $\mathrm{Mol}$ Gastroenterol Hepatol 382:727.

Kujala VJ, Pasqualini FS, Goss JA, Nawroth JC, and Parker KK (2016) Laminar ventricular myocardium on a microelectrode array-based chip. J Mater Chem B Mater Biol Med 4:3534-3543.

Kyttälä A, Moraghebi R, Valensisi C, Kettunen J, Andrus C, Pasumarthy KK, Nakanishi M, Nishimura K, Ohtaka M, Weltner J et al. (2016) Genetic variability overrides the impact of parental cell type and determines iPSC differentiation potential. Stem Cell Reports 6:200-212.

LaBarge W, Mattappally S, Kannappan R, Fast VG, Pretorius D, Berry JL, and Zhang J (2019) Maturation of three-dimensional, hiPSC-derived cardiomyocyte spheroids utilizing cyclic, uniaxial stretch and electrical stimulation. PLoS One 14:e0219442

Lamers MM, Beumer J, van der Vaart J, Knoops K, Puschhof J, Breugem TI, Ravelli RBG, Paul van Schayck J, Mykytyn AZ, Duimel HQ et al. (2020) SARSCoV-2 productively infects human gut enterocytes. Science 369:50-54.

Lan F, Lee AS, Liang P, Sanchez-Freire V, Nguyen PK, Wang L, Han L, Yen M, Wang Y, Sun N et al. (2013) Abnormal calcium handling properties underlie familial hypertrophic cardiomyopathy pathology in patient-specific induced pluripotent stem cells. Cell Stem Cell 12:101-113.

Lancaster MA, Corsini NS, Wolfinger S, Gustafson EH, Phillips AW, Burkard TR, Otani T, Livesey FJ, and Knoblich JA (2017) Guided self-organization and cortical plate formation in human brain organoids. Nat Biotechnol 35:659-666.

Lancaster MA and Knoblich JA (2014) Generation of cerebral organoids from human pluripotent stem cells. Nat Protoc 9:2329-2340.

Lancaster MA, Renner M, Martin C-A, Wenzel D, Bicknell LS, Hurles ME Homfray T, Penninger JM, Jackson AP, and Knoblich JA (2013) Cerebral organoids model human brain development and microcephaly. Nature 501:373-379.

Landry J, Bernier D, Ouellet C, Goyette R, and Marceau N (1985) Spheroidal aggregate culture of rat liver cells: histotypic reorganization, biomatrix deposition, and maintenance of functional activities. J Cell Biol 101:914-923.

Langlands AJ, Carroll TD, Chen Y, and Näthke I (2018) Chir99021 and Valproic acid reduce the proliferative advantage of Apc mutant cells. Cell Death Dis 9:255.

Lauschke VM (2021) Toxicogenomics of drug induced liver injury - from mechanistic understanding to early prediction. Drug Metab Rev 53:245-252.

Lauschke VM, Hendriks DFG, Bell CC, Andersson TB, and Ingelman-Sundberg M (2016a) Novel 3D culture systems for studies of human liver function and assessments of the hepatotoxicity of drugs and drug candidates. Chem Res Toxicol 29:1936-1955.
Lauschke VM and Ingelman-Sundberg M (2016) The importance of patient-specific factors for hepatic drug response and toxicity. Int J Mol Sci 17:1714.

Lauschke VM, Vorrink SU, Moro SML, Rezayee F, Nordling A, Hendriks DFG, Bell CC, Sison-Young R, Park BK, Goldring CE et al. (2016b) Massive rearrangements of cellular MicroRNA signatures are key drivers of hepatocyte dedifferentiation. Hepatology 64:1743-1756.

Lauschke VM, Shafagh RZ, Hendriks DFG, and Ingelman-Sundberg M (2019) 3D primary hepatocyte culture systems for analyses of liver diseases, drug metabolism, and toxicity: emerging culture paradigms and applications. Biotechnol J 14:e1800347.

LaValley DJ, Miller PG, and Shuler ML (2021) Pumpless, unidirectional microphysiological system for testing metabolism-dependent chemotherapeutic toxicity. Biotechnol Prog 37:e3105.

Lee C-T, Chen J, Kindberg AA, Bendriem RM, Spivak CE, Williams MP, Richie CT, Handreck A, Mallon BS, Lupica CR et al. (2017a) CYP3A5 mediates effects of cocaine on human neocorticogenesis: studies using an in vitro 3D self-organized hPSC model with a single cortex-like unit. Neuropsychopharmacology 42:774-784.

Lee DW, Lee SH, Choi N, and Sung JH (2019) Construction of pancreas-muscleliver microphysiological system (MPS) for reproducing glucose metabolism Biotechnol Bioeng 116:3433-3445.

Lee H, Kim DS, Ha SK, Choi I, Lee JM, and Sung JH (2017b) A pumpless multiorgan-on-a-chip (MOC) combined with a pharmacokinetic-pharmacodynamic (PK-PD) model. Biotechnol Bioeng 114:432-443.

Lee H-K, Velazquez Sanchez C, Chen M, Morin PJ, Wells JM, Hanlon EB, and Xia W (2016) Three dimensional human neuro-spheroid model of Alzheimer's disease based on differentiated induced pluripotent stem cells. PLoS One 11:e0163072.

Lee J, Kim SH, Kim Y-C, Choi I, and Sung JH (2013a) Fabrication and characterization of microfluidic liver-on-a-chip using microsomal enzymes. Enzyme Microb Technol 53:159-164.

Lee JB, Zgair A, Taha DA, Zang X, Kagan L, Kim TH, Kim MG, Yun HY, Fischer PM, and Gershkovich P (2017c) Quantitative analysis of lab-to-lab variability in Caco-2 permeability assays. Eur J Pharm Biopharm 114:38-42.

Lee P, Klos M, Bollensdorff C, Hou L, Ewart P, Kamp TJ, Zhang J, Bizy A, Guerrero-Serna G, Kohl P et al. (2012) Simultaneous voltage and calcium mapping of genetically purified human induced pluripotent stem cell-derived cardiac myocyte monolayers. Circ Res 110:1556-1563.

Lee SML, Schelcher C, Demmel M, Hauner M, and Thasler WE (2013b) Isolation of human hepatocytes by a two-step collagenase perfusion procedure. $J$ Vis Exp 79:50615

Lee-Montiel FT, George SM, Gough AH, Sharma AD, Wu J, DeBiasio R, Vernetti LA, and Taylor DL (2017) Control of oxygen tension recapitulates zone-specific functions in human liver microphysiology systems. Exp Biol Med (Maywood) 242:1617-1632

Leite SB, Wilk-Zasadna I, Zaldivar JM, Airola E, Reis-Fernandes MA, Mennecozzi M, Guguen-Guillouzo C, Chesné C, Guillou C, Alves PM et al. (2012) Threedimensional HepaRG model as an attractive tool for toxicity testing. Toxicol Sci 130:106-116.

Lennernäs H, Palm K, Fagerholm U, and Artursson P (1996) Comparison between active and passive drug transport in human intestinal epithelial (Caco-2) cells in vitro and human jejunum in vivo. Int $J$ Pharm 127:103-107.

Leslie JL, Huang S, Opp JS, Nagy MS, Kobayashi M, Young VB, and Spence JR (2015) Persistence and toxin production by Clostridium difficile within human intestinal organoids result in disruption of epithelial paracellular barrier function. Infect Immun 83:138-145.

Li F, Cao L, Parikh S, and Zuo R (2020) Three-dimensional spheroids with primary human liver cells and differential roles of Kupffer cells in drug-induced liver injury. J Pharm Sci 109:1912-1923.

Li GN, Livi LL, Gourd CM, Deweerd ES, and Hoffman-Kim D (2007) Genomic and morphological changes of neuroblastoma cells in response to three-dimensional matrices. Tissue Eng 13:1035-1047.

Li J, Minami I, Yu L, Tsuji K, Nakajima M, Qiao J, Suzuki M, Shimono K Nakatsuji N, Kotera $\mathrm{H}$ et al. (2016a) Extracellular recordings of patterned human pluripotent stem cell-derived cardiomyocytes on aligned fibers. Stem Cells Int 2016:2634013.

Li M, de Graaf IAM, and Groothuis GMM (2016b) Precision-cut intestinal slices: alternative model for drug transport, metabolism, and toxicology research. Expert Opin Drug Metab Toxicol 12:175-190.

Li N, Bi Y-A, Duignan DB, and Lai Y (2009) Quantitative expression profile of hepatobiliary transporters in sandwich cultured rat and human hepatocytes. $\mathrm{Mol}$ Pharm 6:1180-1189.

Li X, George SM, Vernetti L, Gough AH, and Taylor DL (2018a) A glass-based, continuously zonated and vascularized human liver acinus microphysiological system (vLAMPS) designed for experimental modeling of diseases and ADME/ TOX. Lab Chip 18:2614-2631.

Li Y, Kandasamy K, Chuah JKC, Lam YN, Toh WS, Oo ZY, and Zink D (2014) Identification of nephrotoxic compounds with embryonic stem-cell-derived human renal proximal tubular-like cells. Mol Pharm 11:1982-1990.

Li Y, Liu Y, Liu B, Wang J, Wei S, Qi Z, Wang S, Fu W, and Chen Y-G (2018b) A growth factor-free culture system underscores the coordination between Wnt and BMP signaling in Lgr5 ${ }^{+}$intestinal stem cell maintenance. Cell Discov 4:49.

Li Y, Muffat J, Omer A, Bosch I, Lancaster MA, Sur M, Gehrke L, Knoblich JA and Jaenisch R (2017) Induction of expansion and folding in human cerebral organoids. Cell Stem Cell 20:385-396.e3.

Li Y, Oo ZY, Chang SY, Huang P, Eng KG, Zeng JL, Kaestli AJ, Gopalan B, Kandasamy K, Tasnim F et al. (2013) An in vitro method for the prediction of renal proximal tubular toxicity in humans. Toxicol Res (Camb) 2:352-365.

Li Z, Jiang L, Zhu Y, Su W, Xu C, Tao T, Shi Y, and Qin J (2018c) Assessment of hepatic metabolism-dependent nephrotoxicity on an organs-on-a-chip microdevice. Toxicol In Vitro 46:1-8.

Liaw NY and Zimmermann W-H (2016) Mechanical stimulation in the engineering of heart muscle. Adv Drug Deliv Rev 96:156-160 
Lin C and Khetani SR (2016) Advances in engineered liver models for investigating drug-induced liver injury. BioMed Res Int 2016:1829148.

Lin C, Shi J, Moore A, and Khetani SR (2016) Prediction of drug clearance and drug-drug interactions in microscale cultures of human hepatocytes. Drug Metab Dispos 44:127-136.

Lin NYC, Homan KA, Robinson SS, Kolesky DB, Duarte N, Moisan A, and Lewis JA (2019) Renal reabsorption in 3D vascularized proximal tubule models. Proc Natl Acad Sci USA 116:5399-5404.

Lind JU, Busbee TA, Valentine AD, Pasqualini FS, Yuan H, Yadid M, Park S-J, Kotikian A, Nesmith AP, Campbell PH et al. (2017a) Instrumented cardiac microphysiological devices via multimaterial three-dimensional printing. Nat Mater 16:303-308.

Lind JU, Yadid M, Perkins I, O'Connor BB, Eweje F, Chantre CO, Hemphill MA, Yuan H, Campbell PH, Vlassak JJ et al. (2017b) Cardiac microphysiological devices with flexible thin-film sensors for higher-throughput drug screening. Lab Chip 17:3692-3703.

Linder P, Trzewik J, Rüffer M, Artmann GM, Digel I, Kurz R, Rothermel A, Robitzki A, and Temiz Artmann A (2010) Contractile tension and beating rates of self-exciting monolayers and 3D-tissue constructs of neonatal rat cardiomyocytes. Med Biol Eng Comput 48:59-65.

Little MH, Hale LJ, Howden SE, and Kumar SV (2019) Generating kidney from stem cells. Annu Rev Physiol 81:335-357.

Liu J, Li R, Xue R, Li T, Leng L, Wang Y, Wang J, Ma J, Yan J, Yan F et al. (2018a) Liver extracellular matrices bioactivated hepatic spheroids as a model system for drug hepatotoxicity evaluations. Adv Biosyst 2:1800110-1800114.

Liu W, Song J, Du X, Zhou Y, Li Y, Li R, Lyu L, He Y, Hao J, Ben J et al. (2019) AKR1B10 (Aldo-keto reductase family $1 \mathrm{~B} 10$ ) promotes brain metastasis of lung cancer cells in a multi-organ microfluidic chip model. Acta Biomater 91:195-208.

Liu X, Chism JP, LeCluyse EL, Brouwer KR, and Brouwer KL (1999a) Correlation of biliary excretion in sandwich-cultured rat hepatocytes and in vivo in rats. Drug Metab Dispos 27:637-644.

Liu X, LeCluyse EL, Brouwer KR, Lightfoot RM, Lee JI, and Brouwer KL (1999b) Use of $\mathrm{Ca} 2+$ modulation to evaluate biliary excretion in sandwich-cultured rat hepatocytes. J Pharmacol Exp Ther 289:1592-1599.

Liu Y, Qi Z, Li X, Du Y, and Chen Y-G (2018b) Monolayer culture of intestinal epithelium sustains Lgr5 ${ }^{+}$intestinal stem cells. Cell Discov 4:32.

Long TJ, Cosgrove PA, Dunn 2nd RT, Stolz DB, Hamadeh H, Afshari C, McBride H, and Griffith LG (2016) Modeling therapeutic antibody-small molecule drugdrug interactions using a three-dimensional perfusable human liver coculture platform. Drug Metab Dispos 44:1940-1948.

Louisse J, Alewijn M, Peijnenburg AACM, Cnubben NHP, Heringa MB, Coecke S, and Punt A (2020) Towards harmonization of test methods for in vitro hepatic clearance studies. Toxicol In Vitro 63:104722.

Lü S, Liu S, He W, Duan C, Li Y, Liu Z, Zhang Y, Hao T, Wang Y, Li D et al. (2008) Bioreactor cultivation enhances NTEB formation and differentiation of NTES cells into cardiomyocytes. Cloning Stem Cells 10:363-370.

Ma L, Barker J, Zhou C, Li W, Zhang J, Lin B, Foltz G, Küblbeck J, and Honkakoski P (2012) Towards personalized medicine with a three-dimensional micro-scale perfusion-based two-chamber tissue model system. Biomaterials 33:4353-4361.

Ma L-D, Wang Y-T, Wang J-R, Wu J-L, Meng X-S, Hu P, Mu X, Liang Q-L, and Luo G-A (2018) Design and fabrication of a liver-on-a-chip platform for convenient, highly efficient, and safe in situ perfusion culture of 3D hepatic spheroids. Lab Chip 18:2547-2562.

Ma X, Dewan S, Liu J, Tang M, Miller KL, Yu C, Lawrence N, McCulloch AD, and Chen S (2019) 3D printed micro-scale force gauge arrays to improve human cardiac tissue maturation and enable high throughput drug testing. Acta Biomater 95:319-327.

Machida S, Spangenburg EE, and Booth FW (2004) Primary rat muscle progenitor cells have decreased proliferation and myotube formation during passages. Cell Prolif 37:267-277.

Madden L, Juhas M, Kraus WE, Truskey GA, and Bursac N (2015) Bioengineered human myobundles mimic clinical responses of skeletal muscle to drugs. eLife 4:e04885.

Maffioletti SM, Sarcar S, Henderson ABH, Mannhardt I, Pinton L, Moyle LA, Steele-Stallard H, Cappellari O, Wells KE, Ferrari G et al. (2018) Threedimensional human iPSC-derived artificial skeletal muscles model muscular dystrophies and enable multilineage tissue engineering. Cell Rep 23:899-908.

Malan D, Zhang M, Stallmeyer B, Müller J, Fleischmann BK, Schulze-Bahr E, Sasse P, and Greber B (2016) Human iPS cell model of type 3 long QT syndrome recapitulates drug-based phenotype correction. Basic Res Cardiol 111:14.

Mali P, Sarkar A, and Lal R (2006) Facile fabrication of microfluidic systems using electron beam lithography. Lab Chip 6:310-315.

Mancha Sánchez E, Gómez-Blanco JC, López Nieto E, Casado JG, Macías-García A, Díaz Díez MA, Carrasco-Amador JP, Torrejón Martín D, Sánchez-Margallo FM, and Pagador JB (2020) Hydrogels for bioprinting: a systematic review of hydrogels synthesis, bioprinting parameters, and bioprinted structures behavior. Front Bioeng Biotechnol 8:776.

Mansour AA, Gonçalves JT, Bloyd CW, Li H, Fernandes S, Quang D, Johnston S, Parylak SL, Jin X, and Gage FH (2018) An in vivo model of functional and vascularized human brain organoids. Nat Biotechnol 36:432-441.

Maoz BM, Herland A, FitzGerald EA, Grevesse T, Vidoudez C, Pacheco AR, Sheehy SP, Park T-E, Dauth S, Mannix R et al. (2018) A linked organ-on-chip model of the human neurovascular unit reveals the metabolic coupling of endothelial and neuronal cells. Nat Biotechnol 36:865-874.

March S, Ng S, Velmurugan S, Galstian A, Shan J, Logan DJ, Carpenter AE, Thomas D, Sim BKL, Mota MM et al. (2013) A microscale human liver platform that supports the hepatic stages of Plasmodium falciparum and vivax. Cell Host Microbe 14:104-115.
Marchiando AM, Shen L, Graham WV, Weber CR, Schwarz BT, Austin 2nd JR, Raleigh DR, Guan Y, Watson AJM, Montrose MH et al. (2010) Caveolin-1dependent occludin endocytosis is required for TNF-induced tight junction regulation in vivo. J Cell Biol 189:111-126.

Mariani J, Coppola G, Zhang P, Abyzov A, Provini L, Tomasini L, Amenduni M, Szekely A, Palejev D, Wilson M et al. (2015) FOXG1-dependent dysregulation of GABA/glutamate neuron differentiation in autism spectrum disorders. Cell 162:375-390.

Marion TL, Leslie EM, and Brouwer KLR (2007) Use of sandwich-cultured hepatocytes to evaluate impaired bile acid transport as a mechanism of druginduced hepatotoxicity. Mol Pharm 4:911-918.

Marion TL, Perry CH, St Claire 3rd RL, and Brouwer KLR (2012) Endogenous bile acid disposition in rat and human sandwich-cultured hepatocytes. Toxicol Appl Pharmacol 261:1-9.

Marsano A, Conficconi C, Lemme M, Occhetta P, Gaudiello E, Votta E, Cerino G, Redaelli A, and Rasponi M (2016) Beating heart on a chip: a novel microfluidic platform to generate functional 3D cardiac microtissues. Lab Chip 16:599-610.

Martignoni M, Groothuis GMM, and de Kanter R (2006) Species differences between mouse, rat, dog, monkey and human CYP-mediated drug metabolism, inhibition and induction. Expert Opin Drug Metab Toxicol 2:875-894.

Maschmeyer I, Lorenz AK, Schimek K, Hasenberg T, Ramme AP, Hübner J, Lindner M, Drewell C, Bauer S, Thomas A et al. (2015) A four-organ-chip for interconnected long-term co-culture of human intestine, liver, skin and kidney equivalents. Lab Chip 15:2688-2699.

Mastikhina O, Moon B-U, Williams K, Hatkar R, Gustafson D, Mourad O, Sun X, Koo M, Lam AYL, Sun Y et al. (2020) Human cardiac fibrosis-on-a-chip model recapitulates disease hallmarks and can serve as a platform for drug testing. Biomaterials 233:119741.

Mathur A, Loskill P, Shao K, Huebsch N, Hong S, Marcus SG, Marks N, Mandegar M, Conklin BR, Lee LP et al. (2015) Human iPSC-based cardiac microphysiological system for drug screening applications. Sci Rep 5:8883.

McGill MR, Williams CD, Xie Y, Ramachandran A, and Jaeschke H (2012) Acetaminophen-induced liver injury in rats and mice: comparison of protein adducts, mitochondrial dysfunction, and oxidative stress in the mechanism of toxicity. Toxicol Appl Pharmacol 264:387-394.

McKeithan WL, Savchenko A, Yu MS, Cerignoli F, Bruyneel AAN, Price JH, Colas AR, Miller EW, Cashman JR, and Mercola M (2017) An automated platform for assessment of congenital and drug-induced arrhythmia with hiPSC-derived cardiomyocytes. Front Physiol 8:766.

Meier F, Freyer N, Brzeszczynska J, Knöspel F, Armstrong L, Lako M, Greuel S, Damm G, Ludwig-Schwellinger E, Deschl U et al. (2017) Hepatic differentiation of human iPSCs in different 3D models: a comparative study. Int $J \mathrm{Mol} \mathrm{Med}$ 40:1759-1771.

Meinl W, Ebert B, Glatt H, and Lampen A (2008) Sulfotransferase forms expressed in human intestinal Caco-2 and TC7 cells at varying stages of differentiation and role in benzo[a]pyrene metabolism. Drug Metab Dispos 36:276-283.

Mellios N, Feldman DA, Sheridan SD, Ip JPK, Kwok S, Amoah SK, Rosen B, Rodriguez BA, Crawford B, Swaminathan R et al. (2018) MeCP2-regulated miRNAs control early human neurogenesis through differential effects on ERK and AKT signaling. Mol Psychiatry 23:1051-1065

Messner S, Agarkova I, Moritz W, and Kelm JM (2013) Multi-cell type human liver microtissues for hepatotoxicity testing. Arch Toxicol 87:209-213.

Messner S, Fredriksson L, Lauschke VM, Roessger K, Escher C, Bober M, Kelm JM, Ingelman-Sundberg M, and Moritz W (2018) Transcriptomic, proteomic, and functional long-term characterization of multicellular three-dimensional human liver microtissues. Appl In Vitro Toxicol 4:1-12.

Meyer T, Boven KH, Günther E, and Fejtl M (2004) Micro-electrode arrays in cardiac safety pharmacology: a novel tool to study QT interval prolongation. Drug Saf 27:763-772.

Mills RJ, Titmarsh DM, Koenig X, Parker BL, Ryall JG, Quaife-Ryan GA, Voges HK, Hodson MP, Ferguson C, Drowley L et al. (2017) Functional screening in human cardiac organoids reveals a metabolic mechanism for cardiomyocyte cell cycle arrest. Proc Natl Acad Sci USA 114:E8372-E8381.

Mingoia RT, Nabb DL, Yang C-H, and Han X (2007) Primary culture of rat hepatocytes in 96-well plates: effects of extracellular matrix configuration on cytochrome P450 enzyme activity and inducibility, and its application in in vitro cytotoxicity screening. Toxicol In Vitro 21:165-173.

Mladěnka P, Applová L, Patočka J, Costa VM, Remiao F, Pourová J, Mladěnka A, Karlíčková J, Jahodáŕ L, Vopršalová $M$ et al.; TOX-OER and CARDIOTOX Hradec Králové Researchers and Collaborators (2018) Comprehensive review of cardiovascular toxicity of drugs and related agents. Med Res Rev 38:1332-1403.

Mofazzal Jahromi MA, Abdoli A, Rahmanian M, Bardania H, Bayandori M, Moosavi Basri SM, Kalbasi A, Aref AR, Karimi M, and Hamblin MR (2019) Microfluidic brain-on-a-chip: perspectives for mimicking neural system disorders. Mol Neurobiol 56:8489-8512.

Monteil V, Dyczynski M, Lauschke VM, Kwon H, Wirnsberger G, Youhanna S, Zhang H, Slutsky AS, Hurtado Del Pozo C, Horn M et al. (2021) Human soluble ACE2 improves the effect of remdesivir in SARS-CoV-2 infection. EMBO Mol Med 13:e13426.

Moon C, VanDussen KL, Miyoshi H, and Stappenbeck TS (2014) Development of a primary mouse intestinal epithelial cell monolayer culture system to evaluate factors that modulate IgA transcytosis. Mucosal Immunol 7:818-828.

Moorefield EC, Blue RE, Quinney NL, Gentzsch M, and Ding S (2018) Generation of renewable mouse intestinal epithelial cell monolayers and organoids for functional analyses. BMC Cell Biol 19:15.

Morgantini C, Jager J, Li X, Levi L, Azzimato V, Sulen A, Barreby E, Xu C, Tencerova M, Näslund E et al. (2019) Liver macrophages regulate systemic metabolism through non-inflammatory factors. Nat Metab 1:445-459. 
Morizane R, Lam AQ, Freedman BS, Kishi S, Valerius MT, and Bonventre JV (2015) Nephron organoids derived from human pluripotent stem cells model kidney development and injury. Nat Biotechnol 33:1193-1200.

Morrissey KM, Stocker SL, Wittwer MB, Xu L, and Giacomini KM (2013) Renal transporters in drug development. Annu Rev Pharmacol Toxicol 53:503-529.

Mosqueira D, Mannhardt I, Bhagwan JR, Lis-Slimak K, Katili P, Scott E, Hassan M, Prondzynski M, Harmer SC, Tinker A et al. (2018) CRISPR/Cas9 editing in human pluripotent stem cell-cardiomyocytes highlights arrhythmias, hypocontractility, and energy depletion as potential therapeutic targets for hypertrophic cardiomyopathy. Eur Heart J 39:3879-3892.

Mueller D, Koetemann A and Noor F (2011) Organotypic Cultures of Hepg2 Cells for In Vitro Toxicity Studies. Journal of Bioengineering and Biomedical Sciences S2:002.

Mueller D, Krämer L, Hoffmann E, Klein S, and Noor F (2014) 3D organotypic HepaRG cultures as in vitro model for acute and repeated dose toxicity studies. Toxicol In Vitro 28:104-112.

Muffat J, Li Y, Yuan B, Mitalipova M, Omer A, Corcoran S, Bakiasi G, Tsai L-H, Aubourg P, Ransohoff RM et al. (2016) Efficient derivation of microglia-like cells from human pluripotent stem cells. Nat Med 22:1358-1367.

Muguruma K, Nishiyama A, Kawakami H, Hashimoto K, and Sasai Y (2015) Selforganization of polarized cerebellar tissue in $3 \mathrm{D}$ culture of human pluripotent stem cells. Cell Rep 10:537-550.

Mukund K and Subramaniam S (2020) Skeletal muscle: a review of molecular structure and function, in health and disease. Wiley Interdiscip Rev Syst Biol Med 12:e1462.

Mullard A (2021) 2020 FDA drug approvals. Nat Rev Drug Discov 20:85-90.

Müller FA and Sturla SJ (2019) Human in vitro models of nonalcoholic fatty liver disease. Curr Opin Toxicol 16:9-16.

Murakami K, Ishii K, Ishihara Y, Yoshizaki S, Tanaka K, Gotoh Y, Aizaki H, Kohara M, Yoshioka H, Mori Y et al. (2006) Production of infectious hepatitis C virus particles in three-dimensional cultures of the cell line carrying the genomelength dicistronic viral RNA of genotype 1b. Virology 351:381-392.

Murayama N, Usui T, Slawny N, Chesné C, and Yamazaki H (2015) Human HepaRG cells can be cultured in hanging-drop plates for cytochrome P450 induction and function assays. Drug Metab Lett 9:3-7.

Murphy SV and Atala A (2014) 3D bioprinting of tissues and organs. Nat Biotechnol 32:773-785.

Musah S, Mammoto A, Ferrante TC, Jeanty SSF, Hirano-Kobayashi M, Mammoto T, Roberts K, Chung S, Novak R, Ingram M, Fatanat-Didar T, Koshy S, Weaver JC, Church GM and Ingber DE (2017) Mature induced-pluripotent-stem-cellderived human podocytes reconstitute kidney glomerular-capillary-wall function on a chip. Nature Biomedical Engineering 1:0069.

Nam M-O, Hahn S, Jee JH, Hwang T-S, Yoon H, Lee DH, Kwon M-S, and Yoo J (2017) Effects of a small molecule R-spondin-1 substitute RS-246204 on a mouse intestinal organoid culture. Oncotarget 9:6356-6368.

Nam Y-H, Lee S-K, Kim J-H, and Park J-H (2019) PDMS membrane filter with nano-slit array fabricated using three-dimensional silicon mold for the concentration of particles with bacterial size range. Microelectron Eng 215:111008.

Nanki K, Fujii M, Shimokawa M, Matano M, Nishikori S, Date S, Takano A Toshimitsu K, Ohta Y, Takahashi S et al. (2020) Somatic inflammatory gene mutations in human ulcerative colitis epithelium. Nature 577:254-259.

Narayanan K, Schumacher KM, Tasnim F, Kandasamy K, Schumacher A, Ni M, Gao S, Gopalan B, Zink D, and Ying JY (2013) Human embryonic stem cells differentiate into functional renal proximal tubular-like cells. Kidney Int 83:593-603.

Nelson LJ, Morgan K, Treskes P, Samuel K, Henderson CJ, LeBled C, Homer N, Grant MH, Hayes PC, and Plevris JN (2017) Human hepatic HepaRG cells maintain an organotypic phenotype with high intrinsic CYP450 activity/ metabolism and significantly outperform standard HepG2/C3A cells for pharmaceutical and therapeutic applications. Basic Clin Pharmacol Toxicol 120:30-37.

Nemani KV, Moodie KL, Brennick JB, Su A, and Gimi B (2013) In vitro and in vivo evaluation of SU-8 biocompatibility. Mater Sci Eng C 33:4453-4459.

Ng CP, Zhuang Y, Lin A, and Teo JC (2013) A fibrin-based tissue-engineered renal proximal tubule for bioartificial kidney devices: development, characterization and in vitro transport study. Int $J$ Tissue Eng 2013:319476.

Ng S, Schwartz RE, March S, Galstian A, Gural N, Shan J, Prabhu M, Mota MM, and Bhatia SN (2015) Human iPSC-derived hepatocyte-like cells support plasmodium liver-stage infection in vitro. Stem Cell Reports 4:348-359.

Nguemo F, Šarić T, Pfannkuche K, Watzele M, Reppel M, and Hescheler J (2012) In vitro model for assessing arrhythmogenic properties of drugs based on highresolution impedance measurements. Cell Physiol Biochem 29:819-832.

Nguyen DG, Funk J, Robbins JB, Crogan-Grundy C, Presnell SC, Singer T, and Roth $\mathrm{AB}$ (2016) Bioprinted 3D primary liver tissues allow assessment of organlevel response to clinical drug induced toxicity in vitro. PLoS One 11:e0158674.

Nguyen TV, Ukairo O, Khetani SR, McVay M, Kanchagar C, Seghezzi W, Ayanoglu G, Irrechukwu O, and Evers R (2015) Establishment of a hepatocyte-kupffer cell coculture model for assessment of proinflammatory cytokine effects on metabolizing enzymes and drug transporters. Drug Metab Dispos 43:774-785.

Nieskens TTG, Persson M, Kelly EJ, and Sjögren A-K (2020) A multicompartment human kidney proximal tubule-on-a-chip replicates cell polarization-dependent cisplatin toxicity. Drug Metab Dispos 48:1303-1311.

Nigam SK, Wu W, Bush KT, Hoenig MP, Blantz RC, and Bhatnagar V (2015) Handling of drugs, metabolites, and uremic toxins by kidney proximal tubule drug transporters. Clin J Am Soc Nephrol 10:2039-2049.

Noel G, Baetz NW, Staab JF, Donowitz M, Kovbasnjuk O, Pasetti MF, and Zachos NC (2017) A primary human macrophage-enteroid co-culture model to investigate mucosal gut physiology and host-pathogen interactions. Sci Rep 7:45270.
Norona LM, Nguyen DG, Gerber DA, Presnell SC, Mosedale M, and Watkins PB (2019) Bioprinted liver provides early insight into the role of Kupffer cells in TGF- $\beta 1$ and methotrexate-induced fibrogenesis. PLoS One 14:e0208958.

Novik E, Maguire TJ, Chao P, Cheng KC, and Yarmush ML (2010) A microfluidic hepatic coculture platform for cell-based drug metabolism studies. Biochem Pharmacol 79:1036-1044.

Novik EI, Dwyer J, Morelli JK, Parekh A, Cho C, Pludwinski E, Shrirao A Freedman RM, MacDonald JS, and Jayyosi Z (2017) Long-enduring primary hepatocyte-based co-cultures improve prediction of hepatotoxicity. Toxicol Appl Pharmacol 336:20-30.

Novo P, Dell'Aica M, Jender M, Höving S, Zahedi RP, and Janasek D (2017) Integration of polycarbonate membranes in microfluidic free-flow electrophoresis. Analyst (Lond) 142:4228-4239.

Nudischer R, Renggli K, Bertinetti-Lapatki C, Hoflack J-C, Flint N, Sewing S, Pedersen L, Schadt S, Higgins LG, Vardy A et al. (2020) Combining in vivo and organotypic in vitro approaches to assess the human relevance of basimglurant (RG7090), a potential CAR activator. Toxicol Sci 176:329-342

Nunes PS, Ohlsson PD, Ordeig O, and Kutter JP (2010) Cyclic olefin polymers: emerging materials for lab-on-a-chip applications. Microfluid Nanofluidics 9:145-161.

Nzou G, Wicks RT, Wicks EE, Seale SA, Sane CH, Chen A, Murphy SV, Jackson JD, and Atala A.J (2018) Human cortex spheroid with a functional blood brain barrier for high-throughput neurotoxicity screening and disease modeling. Sci Rep 8:7413.

Odawara A, Gotoh M, and Suzuki I (2013) A three-dimensional neuronal culture technique that controls the direction of neurite elongation and the position of soma to mimic the layered structure of the brain. RSC Advances 3:23620-23630.

Ogawa J, Pao GM, Shokhirev MN, and Verma IM (2018) Glioblastoma model using human cerebral organoids. Cell Rep 23:1220-1229.

Ohkura T, Ohta K, Nagao T, Kusumoto K, Koeda A, Ueda T, Jomura T, Ikeya T, Ozeki E, Wada K et al. (2014) Evaluation of human hepatocytes cultured by threedimensional spheroid systems for drug metabolism. Drug Metab Pharmacokinet 29:373-378.

Ölander M, Wiśniewski JR, Matsson P, Lundquist P, and Artursson P (2016) The proteome of filter-grown Caco-2 cells with a focus on proteins involved in drug disposition. J Pharm Sci 105:817-827.

Oleaga C, Riu A, Rothemund S, Lavado A, McAleer CW, Long CJ, Persaud K, Narasimhan NS, Tran M, Roles J et al. (2018) Investigation of the effect of hepatic metabolism on off-target cardiotoxicity in a multi-organ human-on-a-chip system. Biomaterials 182:176-190.

Oliva-Vilarnau N, Vorrink SU, Ingelman-Sundberg M, and Lauschke VM (2020) A $3 \mathrm{D}$ cell culture model identifies Wnt/ $\beta$-catenin mediated inhibition of p53 as a critical step during human hepatocyte regeneration. Adv Sci (Weinh) 7:2000248

Olson H, Betton G, Robinson D, Thomas K, Monro A, Kolaja G, Lilly P, Sanders J, Sipes G, Bracken W et al. (2000) Concordance of the toxicity of pharmaceuticals in humans and in animals. Regul Toxicol Pharmacol 32:56-67.

Onakpoya IJ, Heneghan CJ, and Aronson JK (2016) Post-marketing withdrawal of 462 medicinal products because of adverse drug reactions: a systematic review of the world literature. BMC Med 14:10-11.

Onorati M, Li Z, Liu F, Sousa AMM, Nakagawa N, Li M, Dell'Anno MT, Gulden FO, Pochareddy S, Tebbenkamp ATN et al. (2016) Zika virus disrupts phosphoTBK1 localization and mitosis in human neuroepithelial stem cells and radial glia. Cell Rep 16:2576-2592.

Onses MS, Sutanto E, Ferreira PM, Alleyne AG, and Rogers JA (2015) Mechanisms, capabilities, and applications of high-resolution electrohydrodynamic jet printing. Small 11:4237-4266.

Oorts M, Baze A, Bachellier P, Heyd B, Zacharias T, Annaert P, and Richert L (2016) Drug-induced cholestasis risk assessment in sandwich-cultured human hepatocytes. Toxicol In Vitro 34:179-186.

Ootani A, Li X, Sangiorgi E, Ho QT, Ueno H, Toda S, Sugihara H, Fujimoto K, Weissman IL, Capecchi MR et al. (2009) Sustained in vitro intestinal epithelia culture within a Wnt-dependent stem cell niche. Nat Med 15:701-706.

Ormel PR, Vieira de Sá R, van Bodegraven EJ, Karst H, Harschnitz O, Sneeboer MAM, Johansen LE, van Dijk RE, Scheefhals N, Berdenis van Berlekom A et al. (2018) Microglia innately develop within cerebral organoids. Nat Commun 9:4167.

Ortega-Prieto AM, Skelton JK, Wai SN, Large E, Lussignol M, Vizcay-Barrena G, Hughes D, Fleck RA, Thursz M, Catanese MT et al. (2018) 3D microfluidic liver cultures as a physiological preclinical tool for hepatitis B virus infection. Nat Commun 9:682.

Osaki T, Uzel SGM, and Kamm RD (2018) Microphysiological 3D model of amyotrophic lateral sclerosis (ALS) from human iPS-derived muscle cells and optogenetic motor neurons. Sci Adv 4:eaat5847.

Ostapowicz G, Fontana RJ, Schiødt FV, Larson A, Davern TJ, Han SHB, McCashland TM, Shakil AO, Hay JE, Hynan L et al.; U.S. Acute Liver Failure Study Group (2002) Results of a prospective study of acute liver failure at 17 tertiary care centers in the United States. Ann Intern Med 137:947-954.

Ott LM, Ramachandran K, and Stehno-Bittel L (2017) An automated multiplexed hepatotoxicity and CYP induction assay using HepaRG cells in 2D and 3D. SLAS Discov 22:614-625.

Ozbolat IT and Hospodiuk M (2016) Current advances and future perspectives in extrusion-based bioprinting. Biomaterials 76:321-343.

Ozone C, Suga H, Eiraku M, Kadoshima T, Yonemura S, Takata N, Oiso Y, Tsuji T, and Sasai Y (2016) Functional anterior pituitary generated in self-organizing culture of human embryonic stem cells. Nat Commun 7:10351.

Pandya H, Shen MJ, Ichikawa DM, Sedlock AB, Choi Y, Johnson KR, Kim G, Brown MA, Elkahloun AG, Maric D et al. (2017) Differentiation of human and murine induced pluripotent stem cells to microglia-like cells. Nat Neurosci 20:753-759. 
Pannu N and Nadim MK (2008) An overview of drug-induced acute kidney injury. Crit Care Med 36(4, Suppl)S216-S223.

Parent R, Marion M-J, Furio L, Trépo C, and Petit M-A (2004) Origin and characterization of a human bipotent liver progenitor cell line. Gastroenterology 126:1147-1156.

Park J, Wetzel I, Marriott I, Dréau D, D’Avanzo C, Kim DY, Tanzi RE, and Cho H (2018) A 3D human triculture system modeling neurodegeneration and neuroinflammation in Alzheimer's disease. Nat Neurosci 21:941-951.

Park TI-H, Schweder P, Lee K, Dieriks BV, Jung Y, Smyth L, Rustenhoven J, Mee E, Heppner P, Turner C, Curtis MA, Faull RL, Montgomery JM and Dragunow M (2020) Isolation and culture of functional adult human neurons from neurosurgical brain specimens. Brain Communications 2:fcaa171.

Park J-C, Jang S-Y, Lee D, Lee J, Kang U, Chang H, Kim HJ, Han S-H, Seo J, Choi M et al. (2021) A logical network-based drug-screening platform for Alzheimer's disease representing pathological features of human brain organoids. Nat Commun 12:280.

Park S-J, Zhang D, Qi Y, Li Y, Lee KY, Bezzerides VJ, Yang P, Xia S, Kim SL, Liu $\mathrm{X}$ et al. (2019) Insights into the pathogenesis of catecholaminergic polymorphic ventricular tachycardia from engineered human heart tissue. Circulation 140:390-404.

Paşca AM, Sloan SA, Clarke LE, Tian Y, Makinson CD, Huber N, Kim CH, Park J-Y, O'Rourke NA, Nguyen KD et al. (2015) Functional cortical neurons and astrocytes from human pluripotent stem cells in 3D culture. Nat Methods 12:671-678.

Pastuła A, Middelhoff M, Brandtner A, Tobiasch M, Höhl B, Nuber AH, Demir IE, Neupert S, Kollmann P, Mazzuoli-Weber G et al. (2016) Three-dimensional gastrointestinal organoid culture in combination with nerves or fibroblasts: a method to characterize the gastrointestinal stem cell niche. Stem Cells Int 2016:3710836

Peel S, Corrigan AM, Ehrhardt B, Jang K-J, Caetano-Pinto P, Boeckeler M, Rubins JE, Kodella K, Petropolis DB, Ronxhi J et al. (2019) Introducing an automated high content confocal imaging approach for organs-on-chips. Lab Chip 19:410-421.

Peltz G (2013) Can 'humanized' mice improve drug development in the 21st century? Trends Pharmacol Sci 34:255-260.

Pereira M, Pfisterer U, Rylander D, Torper O, Lau S, Lundblad M, Grealish S, and Parmar M (2014) Highly efficient generation of induced neurons from human fibroblasts that survive transplantation into the adult rat brain. Sci Rep 4:6330.

Perel P, Roberts I, Sena E, Wheble P, Briscoe C, Sandercock P, Macleod M, Mignini LE, Jayaram P, and Khan KS (2007) Comparison of treatment effects between animal experiments and clinical trials: systematic review. BMJ 334:197.

Peters MF, Choy AL, Pin C, Leishman DJ, Moisan A, Ewart L, Guzzie-Peck PJ, Sura R, Keller DA, Scott CW et al. (2020) Developing in vitro assays to transform gastrointestinal safety assessment: potential for microphysiological systems. Lab Chip 20:1177-1190.

Peterson NC, Mahalingaiah PK, Fullerton A, and Di Piazza M (2020) Application of microphysiological systems in biopharmaceutical research and development. Lab Chip 20:697-708.

Petropolis DB, Faust DM, Deep Jhingan G, and Guillen N (2014) A new human 3D-liver model unravels the role of galectins in liver infection by the parasite Entamoeba histolytica. PLoS Pathog 10:e1004381.

Petrosyan A, Cravedi P, Villani V, Angeletti A, Manrique J, Renieri A, De Filippo RE, Perin L, and Da Sacco S (2019) A glomerulus-on-a-chip to recapitulate the human glomerular filtration barrier. Nat Commun 10:3656.

Phillips JA, Grandhi TSP, Davis M, Gautier J-C, Hariparsad N, Keller D, Sura R, and Van Vleet TR (2020) A pharmaceutical industry perspective on microphysiological kidney systems for evaluation of safety for new therapies. $L a b$ Chip 20:468-476.

Pingitore P, Sasidharan K, Ekstrand M, Prill S, Lindén D, and Romeo S (2019) Human multilineage 3D spheroids as a model of liver steatosis and fibrosis. Int $J$ Mol Sci 20:1629.

Pleguezuelos-Manzano C, Puschhof J, Rosendahl Huber A, van Hoeck A, Wood HM, Nomburg J, Gurjao C, Manders F, Dalmasso G, Stege PB et al.; Genomics England Research Consortium (2020) Mutational signature in colorectal cancer caused by genotoxic $\mathrm{pks}^{+}$E. coli. Nature 580:269-273

Ploss A, Khetani SR, Jones CT, Syder AJ, Trehan K, Gaysinskaya VA, Mu K, Ritola K, Rice CM, and Bhatia SN (2010) Persistent hepatitis C virus infection in microscale primary human hepatocyte cultures. Proc Natl Acad Sci USA 107:3141-3145.

Pocock K, Delon L, Bala V, Rao S, Priest C, Prestidge C, and Thierry B (2017) Intestine-on-a-chip microfluidic model for efficient in vitro screening of oral chemotherapeutic uptake. ACS Biomater Sci Eng 3:951-959.

Pointon A, Pilling J, Dorval T, Wang Y, Archer C, and Pollard C (2017) From the cover: high-throughput imaging of cardiac microtissues for the assessment of cardiac contraction during drug discovery. Toxicol Sci 155:444-457.

Pointon A, Maher J, Davis M, Baker T, Cichocki J, Ramsden D, Hale C, Kolaja KL, Levesque P, Sura R et al. (2021) Cardiovascular microphysiological systems (CVMPS) for safety studies - a pharma perspective. Lab Chip 21:458-472.

Polonchuk L, Chabria M, Badi L, Hoflack J-C, Figtree G, Davies MJ, and Gentile C (2017) Cardiac spheroids as promising in vitro models to study the human heart microenvironment. Sci Rep 7:7005.

Pontier C, Pachot J, Botham R, Lenfant B, and Arnaud P (2001) HT29-MTX and Caco-2/TC7 monolayers as predictive models for human intestinal absorption: role of the mucus layer. J Pharm Sci 90:1608-1619.

Pouya A, Rassouli H, Rezaei-Larijani M, Salekdeh GH, and Baharvand H (2020) SOX2 protein transduction directly converts human fibroblasts into oligodendrocytelike cells. Biochem Biophys Res Commun 525:1-7.

Prill S, Caddeo A, Baselli G, Jamialahmadi O, Dongiovanni P, Rametta R, Kanebratt KP, Pujia A, Pingitore P, Mancina RM et al. (2019) The TM6SF2
E167K genetic variant induces lipid biosynthesis and reduces apolipoprotein B secretion in human hepatic 3D spheroids. Sci Rep 9:11585.

Prior N, Inacio P, and Huch M (2019) Liver organoids: from basic research to therapeutic applications. Gut 68:2228-2237.

Proctor WR, Foster AJ, Vogt J, Summers C, Middleton B, Pilling MA, Shienson D, Kijanska M, Ströbel S, Kelm JM et al. (2017) Utility of spherical human liver microtissues for prediction of clinical drug-induced liver injury. Arch Toxicol 91:2849-2863.

Prondzynski M, Lemoine MD, Zech AT, Horváth A, Di Mauro V, Koivumäki JT, Kresin N, Busch J, Krause T, Krämer E et al. (2019) Disease modeling of a mutation in $\alpha$-actinin 2 guides clinical therapy in hypertrophic cardiomyopathy. EMBO Mol Med 11:e11115.

Pusch J, Votteler M, Göhler S, Engl J, Hampel M, Walles H, and Schenke-Layland K (2011) The physiological performance of a three-dimensional model that mimics the microenvironment of the small intestine. Biomaterials 32:7469-7478.

Qian T, Maguire SE, Canfield SG, Bao X, Olson WR, Shusta EV, and Palecek SP (2017a) Directed differentiation of human pluripotent stem cells to blood-brain barrier endothelial cells. Sci Adv 3:e1701679.

Qian X, Jacob F, Song MM, Nguyen HN, Song H, and Ming GL (2018) Generation of human brain region-specific organoids using a miniaturized spinning bioreactor. Nat Protoc 13:565-580.

Qian X, Nguyen HN, Jacob F, Song H, and Ming GL (2017b) Using brain organoids to understand Zika virus-induced microcephaly. Development 144:952-957.

Qian X, Nguyen HN, Song MM, Hadiono C, Ogden SC, Hammack C, Yao B, Hamersky GR, Jacob F, Zhong C et al. (2016) Brain-region-specific organoids using mini-bioreactors for modeling ZIKV exposure. Cell 165:1238-1254.

Raimondi MT, Albani D, and Giordano C (2019) An organ-on-a-chip engineered platform to study the microbiota-gut-brain axis in neurodegeneration. Trends Mol Med 25:737-740.

Raja WK, Mungenast AE, Lin Y-T, Ko T, Abdurrob F, Seo J, and Tsai L-H (2016) Self-organizing 3D human neural tissue derived from induced pluripotent stem cells recapitulate Alzheimer's disease phenotypes. PLoS One 11:e0161969.

Rajan A, Vela L, Zeng X-L, Yu X, Shroyer N, Blutt SE, Poole NM, Carlin LG, Nataro JP, Estes MK et al. (2018) Novel segment- and host-specific patterns of enteroaggregative Escherichia coli adherence to human intestinal enteroids. MBio 9:e02419-e17.

Rajan SAP, Aleman J, Wan M, Pourhabibi Zarandi N, Nzou G, Murphy S, Bishop CE, Sadri-Ardekani H, Shupe T, Atala A et al. (2020) Probing prodrug metabolism and reciprocal toxicity with an integrated and humanized multitissue organ-on-a-chip platform. Acta Biomater 106:124-135.

Ramaiahgari SC, den Braver MW, Herpers B, Terpstra V, Commandeur JNM, van de Water B, and Price LS (2014) A 3D in vitro model of differentiated HepG2 cell spheroids with improved liver-like properties for repeated dose high-throughput toxicity studies. Arch Toxicol 88:1083-1095.

Ramaiahgari SC, Waidyanatha S, Dixon D, DeVito MJ, Paules RS, and Ferguson SS (2017) From the cover: three-dimensional (3D) HepaRG spheroid model with physiologically relevant xenobiotic metabolism competence and hepatocyte functionality for liver toxicity screening. Toxicol Sci 159:124-136.

Rao L, Qian Y, Khodabukus A, Ribar T, and Bursac N (2018) Engineering human pluripotent stem cells into a functional skeletal muscle tissue. Nat Commun 9:126.

Rebelo SP, Costa R, Estrada M, Shevchenko V, Brito C, and Alves PM (2015) HepaRG microencapsulated spheroids in DMSO-free culture: novel culturing approaches for enhanced xenobiotic and biosynthetic metabolism. Arch Toxicol 89:1347-1358

Rebelo SP, Costa R, Silva MM, Marcelino P, Brito C, and Alves PM (2017) Threedimensional co-culture of human hepatocytes and mesenchymal stem cells: improved functionality in long-term bioreactor cultures. J Tissue Eng Regen Med 11:2034-2045

Reif R, Karlsson J, Günther G, Beattie L, Wrangborg D, Hammad S, Begher-Tibbe B, Vartak A, Melega S, Kaye PM et al. (2015) Bile canalicular dynamics in hepatocyte sandwich cultures. Arch Toxicol 89:1861-1870.

Reinholz J, Landfester K, and Mailänder V (2018) The challenges of oral drug delivery via nanocarriers. Drug Deliv 25:1694-1705.

Renner H, Grabos M, Becker KJ, Kagermeier TE, Wu J, Otto M, Peischard S, Zeuschner D, TsyTsyura Y, Disse P et al. (2020) A fully automated highthroughput workflow for 3D-based chemical screening in human midbrain organoids. eLife 9:e52904.

Ribeiro AJS, Schwab O, Mandegar MA, Ang Y-S, Conklin BR, Srivastava D, and Pruitt BL (2017) Multi-imaging method to assay the contractile mechanical output of micropatterned human iPSC-derived cardiac myocytes. Circ Res 120:1572-1583.

Richards DJ, Tan Y, Coyle R, Li Y, Xu R, Yeung N, Parker A, Menick DR, Tian B, and Mei Y (2016) Nanowires and electrical stimulation synergistically improve functions of hiPSC cardiac spheroids. Nano Lett 16:4670-4678.

Richardson P, Griffin I, Tucker C, Smith D, Oechsle O, Phelan A, Rawling M, Savory E, and Stebbing J (2020) Baricitinib as potential treatment for 2019$\mathrm{nCoV}$ acute respiratory disease. Lancet 395:e30-e31.

Richert L, Baze A, Parmentier C, Gerets HHJ, Sison-Young R, Dorau M, Lovatt C, Czich A, Goldring C, Park BK et al. (2016) Cytotoxicity evaluation using cryopreserved primary human hepatocytes in various culture formats. Toxicol Lett 258:207-215.

Ridder BJ, Leishman DJ, Bridgland-Taylor M, Samieegohar M, Han X, Wu WW, Randolph A, Tran P, Sheng J, Danker T et al. (2020) A systematic strategy for estimating hERG block potency and its implications in a new cardiac safety paradigm. Toxicol Appl Pharmacol 394:114961.

Riede J, Wollmann BM, Molden E and Ingelman-Sundberg M (2021) Primary human hepatocyte spheroids as an in vitro tool for investigating drug compounds with low clearance. Drug Metabolism and Disposition DOI: 10.1124/dmd.120. 000340 [published ahead of print]. 
Rigat-Brugarolas LG, Elizalde-Torrent A, Bernabeu M, De Niz M, Martin-Jaular L, Fernandez-Becerra C, Homs-Corbera A Samitier J, and del Portillo HA (2014) A functional microengineered model of the human splenon-on-a-chip. Lab Chip 14:1715-1724

Robinson JM, Turkington S, Abey SA, Kenea N, and Henderson WA (2019) Differential gene expression and gene-set enrichment analysis in Caco-2 monolayers during a 30-day timeline with dexamethasone exposure. Tissue Barriers 7:e1651597.

Roeselers G, Ponomarenko M, Lukovac S, and Wortelboer HM (2013) Ex vivo systems to study host-microbiota interactions in the gastrointestinal tract. Best Pract Res Clin Gastroenterol 27:101-113.

Ronaldson-Bouchard K, Ma SP, Yeager K, Chen T, Song L, Sirabella D, Morikawa K, Teles D, Yazawa M, and Vunjak-Novakovic G (2018) Advanced maturation of human cardiac tissue grown from pluripotent stem cells. Nature 556:239-243.

Ronaldson-Bouchard K and Vunjak-Novakovic G (2018) Organs-on-a-chip: a fast track for engineered human tissues in drug development. Cell Stem Cell 22:310-324.

Rubas W, Jezyk N, and Grass GM (1993) Comparison of the permeability characteristics of a human colonic epithelial (Caco-2) cell line to colon of rabbit, monkey, and dog intestine and human drug absorption. Pharm Res 10:113-118.

Rubiano A, Indapurkar A, Yokosawa R, Miedzik A, Rosenzweig B, Arefin A, Moulin CM, Dame K, Hartman N, Volpe DA et al. (2021) Characterizing the reproducibility in using a liver microphysiological system for assaying drug toxicity, metabolism, and accumulation. Clin Transl Sci 14:1049-1061.

Rundqvist J, Mendoza B, Werbin JL, Heinz WF, Lemmon C, Romer LH, Haviland DB, and Hoh JH (2007) High fidelity functional patterns of an extracellular matrix protein by electron beam-based inactivation. J Am Chem Soc 129:59-67.

Sakolish C, Chen Z, Dalaijamts C, Mitra K, Liu Y, Fulton T, Wade TL, Kelly EJ, Rusyn I, and Chiu WA (2020) Predicting tubular reabsorption with a human kidney proximal tubule tissue-on-a-chip and physiologically-based modeling. Toxicol In Vitro 63:104752.

Sakolish CM, Philip B, and Mahler GJ (2019) A human proximal tubule-on-a-chip to study renal disease and toxicity. Biomicrofluidics 13:014107.

Sala L, van Meer BJ, Tertoolen LGJ, Bakkers J, Bellin M, Davis RP, Denning C, Dieben MAE, Eschenhagen T, Giacomelli E et al. (2018) MUSCLEMOTION: a versatile open software tool to quantify cardiomyocyte and cardiac muscle contraction in vitro and in vivo. Circ Res 122:e5-e16.

Saleem U, van Meer BJ, Katili PA, Mohd Yusof NAN, Mannhardt I, Garcia AK, Tertoolen L, de Korte T, Vlaming MLH, McGlynn K et al. (2020) Blinded, multicenter evaluation of drug-induced changes in contractility using humaninduced pluripotent stem cell-derived cardiomyocytes. Toxicol Sci 176:103-123.

Sambuy Y, De Angelis I, Ranaldi G, Scarino ML, Stammati A, and Zucco F (2005) The Caco-2 cell line as a model of the intestinal barrier: influence of cell and culture-related factors on Caco-2 cell functional characteristics. Cell Biol Toxicol 21:1-26.

Sánchez-Romero N, Martínez-Gimeno L, Caetano-Pinto P, Saez B, SánchezZalabardo JM, Masereeuw R, and Giménez I (2020) A simple method for the isolation and detailed characterization of primary human proximal tubule cells for renal replacement therapy. Int J Artif Organs 43:45-57.

Sandström N, Zandi Shafagh R, Vastesson A, Carlborg CF, van der Wijngaart W, and Haraldsson T (2015) Reaction injection molding and direct covalent bonding of OSTE+ polymer microfluidic devices. J Micromech Microeng 25:075002.

Sano E, Mori C, Matsuoka N, Ozaki Y, Yagi K, Wada A, Tashima K, Yamasaki S, Tanabe K, Yano K et al. (2019) Tetrafluoroethylene-propylene elastomer for fabrication of microfluidic organs-on-chips resistant to drug absorption. Micromachines (Basel) 10:793.

Santbergen MJC, van der Zande M, Gerssen A, Bouwmeester H, and Nielen MWF (2020) Dynamic in vitro intestinal barrier model coupled to chip-based liquid chromatography mass spectrometry for oral bioavailability studies. Anal Bioanal Chem 412:1111-1122.

Santhanam N, Kumanchik L, Guo X, Sommerhage F, Cai Y, Jackson M, Martin C, Saad G, McAleer CW, Wang Y et al. (2018) Stem cell derived phenotypic human neuromuscular junction model for dose response evaluation of therapeutics. Biomaterials 166:64-78.

Sato T, Vries RG, Snippert HJ, van de Wetering M, Barker N, Stange DE, van Es JH, Abo A, Kujala P, Peters PJ et al. (2009) Single Lgr5 stem cells build cryptvillus structures in vitro without a mesenchymal niche. Nature 459:262-265.

Satoh T, Sugiura S, Shin K, Onuki-Nagasaki R, Ishida S, Kikuchi K, Kakiki M, and Kanamori T (2017) A multi-throughput multi-organ-on-a-chip system on a plate formatted pneumatic pressure-driven medium circulation platform. $L a b$ Chip 18:115-125.

Schepers A, Li C, Chhabra A, Seney BT, and Bhatia S (2016) Engineering a perfusable 3D human liver platform from iPS cells. Lab Chip 16:2644-2653.

Schimek K, Frentzel S, Luettich K, Bovard D, Rütschle I, Boden L, Rambo F, Erfurth H, Dehne E-M, Winter A et al. (2020) Human multi-organ chip coculture of bronchial lung culture and liver spheroids for substance exposure studies. Sci Rep 10:7865-7813.

Schimpel C, Teubl B, Absenger M, Meindl C, Fröhlich E, Leitinger G, Zimmer A and Roblegg E (2014) Development of an advanced intestinal in vitro triple culture permeability model to study transport of nanoparticles. Mol Pharm 11:808-818.

Schmid YRF, Bürgel SC, Misun PM, Hierlemann A, and Frey O (2016) Electrical impedance spectroscopy for microtissue spheroid analysis in hanging-drop networks. ACS Sens 1:1028-1035.

Schneider O, Zeifang L, Fuchs S, Sailer C, and Loskill P (2019) User-friendly and parallelized generation of human induced pluripotent stem cell-derived microtissues in a centrifugal heart-on-a-chip. Tissue Eng Part A 25:786-798.

Schutgens F, Rookmaaker MB, Margaritis T, Rios A, Ammerlaan C, Jansen J, Gijzen L, Vormann M, Vonk A, Viveen M et al. (2019) Tubuloids derived from human adult kidney and urine for personalized disease modeling. Nat Biotechnol 37:303-313.

Schwartz MP, Hou Z, Propson NE, Zhang J, Engstrom CJ, Santos Costa V, Jiang P, Nguyen BK, Bolin JM, Daly W et al. (2015) Human pluripotent stem cell-derived neural constructs for predicting neural toxicity. Proc Natl Acad Sci USA 112:12516-12521.

Schweinlin M, Wilhelm S, Schwedhelm I, Hansmann J, Rietscher R, Jurowich C, Walles H, and Metzger M (2016) Development of an advanced primary human in vitro model of the small intestine. Tissue Eng Part C Methods 22:873-883.

Scott A, Rouch JD, Jabaji Z, Khalil HA, Solorzano S, Lewis M, Martín MG, Stelzner MG, and Dunn JCY (2016) Long-term renewable human intestinal epithelial stem cells as monolayers: a potential for clinical use. J Pediatr Surg 51:995-1000.

Scott CW, Zhang X, Abi-Gerges N, Lamore SD, Abassi YA, and Peters MF (2014) An impedance-based cellular assay using human iPSC-derived cardiomyocytes to quantify modulators of cardiac contractility. Toxicol Sci 142:331-338.

Sellaro TL, Ranade A, Faulk DM, McCabe GP, Dorko K, Badylak SF, and Strom SC (2010) Maintenance of human hepatocyte function in vitro by liver-derived extracellular matrix gels. Tissue Eng Part A 16:1075-1082.

Sellgren CM, Sheridan SD, Gracias J, Xuan D, Fu T, and Perlis RH (2017) Patientspecific models of microglia-mediated engulfment of synapses and neural progenitors. Mol Psychiatry 22:170-177.

Serras AS, Rodrigues JS, Cipriano M, Rodrigues AV, Oliveira NG, and Miranda JP (2021) A critical perspective on 3D liver models for drug metabolism and toxicology studies. Front Cell Dev Biol 9:626805.

Sgro C, Clinard F, Ouazir K, Chanay H, Allard C, Guilleminet C, Lenoir C, Lemoine A, and Hillon P (2002) Incidence of drug-induced hepatic injuries: a French population-based study. Hepatology 36:451-455

Shah P, Fritz JV, Glaab E, Desai MS, Greenhalgh K, Frachet A, Niegowska M, Estes M, Jäger C, Seguin-Devaux C et al. (2016) A microfluidics-based in vitro model of the gastrointestinal human-microbe interface. Nat Commun 7:11535.

Shah U-K, Mallia JO, Singh N, Chapman KE, Doak SH, and Jenkins GJS (2018) A three-dimensional in vitro HepG2 cells liver spheroid model for genotoxicity studies. Mutat Res Genet Toxicol Environ Mutagen 825:51-58.

Shanks N, Greek R, and Greek J (2009) Are animal models predictive for humans? Philos Ethics Humanit Med 4:2.

Shim K-Y, Lee D, Han J, Nguyen N-T, Park S, and Sung JH (2017) Microfluidic gut-on-a-chip with three-dimensional villi structure. Biomed Microdevices 19:37.

Shin W and Kim HJ (2018) Intestinal barrier dysfunction orchestrates the onset of inflammatory host-microbiome cross-talk in a human gut inflammation-on-achip. Proc Natl Acad Sci USA 115:E10539-E10547.

Shin YC, Shin W, Koh D, Wu A, Ambrosini YM, Min S, Eckhardt SG, Fleming RYD, Kim S, Park S et al. (2020) Three-dimensional regeneration of patientderived intestinal organoid epithelium in a physiodynamic mucosal interface-ona-chip. Micromachines (Basel) 11:663.

Shinha K, Nihei W, Ono T, Nakazato R, and Kimura H (2020) A pharmacokineticpharmacodynamic model based on multi-organ-on-a-chip for drug-drug interaction studies. Biomicrofluidics 14:044108.

Shinnawi R, Huber I, Maizels L, Shaheen N, Gepstein A, Arbel G, Tijsen AJ, and Gepstein L (2015) Monitoring human-induced pluripotent stem cell-derived cardiomyocytes with genetically encoded calcium and voltage fluorescent reporters. Stem Cell Reports 5:582-596.

Shlomai A, Schwartz RE, Ramanan V, Bhatta A, de Jong YP, Bhatia SN, and Rice CM (2014) Modeling host interactions with hepatitis B virus using primary and induced pluripotent stem cell-derived hepatocellular systems. Proc Natl Acad Sci USA 111:12193-12198.

Si-Tayeb K, Noto FK, Nagaoka M, Li J, Battle MA, Duris C, North PE, Dalton S, and Duncan SA (2010) Highly efficient generation of human hepatocyte-like cells from induced pluripotent stem cells. Hepatology 51:297-305.

Sinnecker D, Laugwitz K-L, and Moretti A (2014) Induced pluripotent stem cellderived cardiomyocytes for drug development and toxicity testing. Pharmacol Ther 143:246-252.

Sirenko O, Crittenden C, Callamaras N, Hesley J, Chen Y-W, Funes C, Rusyn I, Anson B, and Cromwell EF (2013) Multiparameter in vitro assessment of compound effects on cardiomyocyte physiology using iPSC cells. J Biomol Screen 18:39-53.

Sirenko O, Hancock MK, Crittenden C, Hammer M, Keating S, Carlson CB, and Chandy G (2017) Phenotypic assays for characterizing compound effects on induced pluripotent stem cell-derived cardiac spheroids. Assay Drug Dev Technol 15:280-296.

Sirenko O, Hancock MK, Hesley J, Hong D, Cohen A, Gentry J, Carlson CB, and Mann DA (2016) Phenotypic characterization of toxic compound effects on liver spheroids derived from iPSC using confocal imaging and three-dimensiona image analysis. Assay Drug Dev Technol 14:381-394.

Sison-Young RL, Lauschke VM, Johann E, Alexandre E, Anthérieu S, Aerts H, Gerets HHJ, Labbe G, Hoët D, Dorau M et al. (2017) A multicenter assessment of single-cell models aligned to standard measures of cell health for prediction of acute hepatotoxicity. Arch Toxicol 91:1385-1400.

Sison-Young RLC, Mitsa D, Jenkins RE, Mottram D, Alexandre E, Richert L, Aert $\mathrm{H}$, Weaver RJ, Jones RP, Johann E et al. (2015) Comparative proteomic characterization of 4 human liver-derived single cell culture models reveals significant variation in the capacity for drug disposition, bioactivation, and detoxication. Toxicol Sci 147:412-424.

Sivitilli AA, Gosio JT, Ghoshal B, Evstratova A, Trcka D, Ghiasi P, Hernandez JJ, Beaulieu JM, Wrana JL, and Attisano L (2020) Robust production of uniform human cerebral organoids from pluripotent stem cells. Life Sci Alliance 3:e202000707.

Skardal A, Aleman J, Forsythe S, Rajan S, Murphy S, Devarasetty M, Pourhabibi Zarandi N, Nzou G, Wicks R, Sadri-Ardekani H et al. (2020) Drug compound 
screening in single and integrated multi-organoid body-on-a-chip systems. Biofabrication 12:025017.

Skardal A, Murphy SV, Devarasetty M, Mead I, Kang H-W, Seol Y-J, Shrike Zhang Y, Shin SR, Zhao L, Aleman J et al. (2017) Multi-tissue interactions in an integrated three-tissue organ-on-a-chip platform. Sci Rep 7:8837.

Smith S, Lyman M, Ma B, Tweedie D and Menzel K (2021) Reaction phenotyping of low-turnover compounds in long-term hepatocyte cultures through persistent selective inhibition of cytochromes P450. Drug Metabolism and Disposition. doi: https://doi.org/10.1124/dmd.121.000601. Online ahead of print.

Song B, Smink AM, Jones CV, Callaghan JM, Firth SD, Bernard CA, Laslett AL, Kerr PG, and Ricardo SD (2012a) The directed differentiation of human iPS cells into kidney podocytes. PLoS One 7:e46453.

Song B, Sun G, Herszfeld D, Sylvain A, Campanale NV, Hirst CE, Caine S, Parkington HC, Tonta MA, Coleman HA et al. (2012b) Neural differentiation of patient specific iPS cells as a novel approach to study the pathophysiology of multiple sclerosis. Stem Cell Res (Amst) 8:259-273.

Song Y, Kumar CSSR, and Hormes J (2004) Fabrication of an SU-8 based microfluidic reactor on a PEEK substrate sealed by a 'flexible semi-solid transfer'(FST) process. J Micromech Microeng 14:932.

Sontheimer-Phelps A, Chou DB, Tovaglieri A, Ferrante TC, Duckworth T, Fadel C, Frismantas V, Sutherland AD, Jalili-Firoozinezhad S, Kasendra M et al. (2020) Human colon-on-a-chip enables continuous in vitro analysis of colon mucus layer accumulation and physiology. Cell Mol Gastroenterol Hepatol 9:507-526.

Soret P-A, Magusto J, Housset C, and Gautheron J (2020) In vitro and in vivo models of non-alcoholic fatty liver disease: a critical appraisal. J Clin Med 10:36.

Spaethling JM, Na Y-J, Lee J, Ulyanova AV, Baltuch GH, Bell T.J, Brem S, Chen HI, Dueck H, Fisher SA et al. (2017) Primary cell culture of live neurosurgically resected aged adult human brain cells and single cell transcriptomics. Cell Rep 18:791-803.

Spence JR, Mayhew CN, Rankin SA, Kuhar MF, Vallance JE, Tolle K, Hoskins EE, Kalinichenko VV, Wells SI, Zorn AM et al. (2011) Directed differentiation of human pluripotent stem cells into intestinal tissue in vitro. Nature 470:105-109.

Stachowiak EK, Benson CA, Narla ST, Dimitri A, Chuye LEB, Dhiman S, Harikrishnan K, Elahi S, Freedman D, Brennand KJ et al. (2017) Cerebral organoids reveal early cortical maldevelopment in schizophrenia-computational anatomy and genomics, role of FGFR1. Transl Psychiatry 7:6

Stebbing J, Krishnan V, de Bono S, Ottaviani S, Casalini G, Richardson PJ, Monteil V, Lauschke VM, Mirazimi A, Youhanna S et al.; Sacco Baricitinib Study Group (2020) Mechanism of baricitinib supports artificial intelligence-predicted testing in COVID-19 patients. EMBO Mol Med 12:e12697.

Stebbing J, Sánchez Nievas G, Falcone M, Youhanna S, Richardson P, Ottaviani S Shen JX, Sommerauer C, Tiseo G, Ghiadoni L et al. (2021) JAK inhibition reduces SARS-CoV-2 liver infectivity and modulates inflammatory responses to reduce morbidity and mortality. Sci Adv 7 :eabe4724.

Steensma A, Noteborn HPJM, and Kuiper HA (2004) Comparison of Caco-2, IEC18 and HCEC cell lines as a model for intestinal absorption of genistein, daidzein and their glycosides. Environ Toxicol Pharmacol 16:131-139.

Stewart BH, Chan OH, Lu RH, Reyner EL, Schmid HL, Hamilton HW, Steinbaugh BA, and Taylor MD (1995) Comparison of intestinal permeabilities determined in multiple in vitro and in situ models: relationship to absorption in humans. Pharm Res 12:693-699.

Stokes CL, Cirit M, and Lauffenburger DA (2015) Physiome-on-a-chip: the challenge of "scaling" in design, operation, and translation of microphysiological systems. CPT Pharmacometrics Syst Pharmacol 4:559-562.

Stuart C and Chen Y (2009) Roll in and roll out: a path to high-throughput nanoimprint lithography. ACS Nano 3:2062-2064

Sun H, Chow EC, Liu S, Du Y, and Pang KS (2008) The Caco-2 cell monolayer: usefulness and limitations. Expert Opin Drug Metab Toxicol 4:395-411.

Sun L, Yin Z, Qi L, Wu D, and Zou H (2016) A low-cost method of fabricating hybrid micro-nano fluidic chip by double-layer PDMS mold and UV-thermal nanoimprinting. Microfluid Nanofluidics 20:1-10

Sun N, Yazawa M, Liu J, Han L, Sanchez-Freire V, Abilez OJ, Navarrete EG, Hu S, Wang L, Lee A et al. (2012) Patient-specific induced pluripotent stem cells as a model for familial dilated cardiomyopathy. Sci Transl Med 4:130ra47.

Sun P, Zhang G, Su X, Jin C, Yu B, Yu X, Lv Z, Ma H, Zhang M, Wei W et al. (2019) Maintenance of primary hepatocyte functions in vitro by inhibiting mechanical tension-induced YAP activation. Cell Rep 29:3212-3222.e4.

Sung JH, Yu J, Luo D, Shuler ML, and March JC (2011) Microscale 3-D hydrogel scaffold for biomimetic gastrointestinal (GI) tract model. Lab Chip 11:389-392.

Susewind J, de Souza Carvalho-Wodarz C, Repnik U, Collnot E-M, SchneiderDaum N, Griffiths GW, and Lehr C-M (2016) A 3D co-culture of three human cell lines to model the inflamed intestinal mucosa for safety testing of nanomaterials. Nanotoxicology 10:53-62.

Suurmond CE, Lasli S, van den Dolder FW, Ung A, Kim H-J, Bandaru P, Lee K, Cho H-J, Ahadian S, Ashammakhi N et al. (2019) In vitro human liver model of nonalcoholic steatohepatitis by coculturing hepatocytes, endothelial cells, and Kupffer cells. Adv Healthc Mater 8:e1901379.

Taguchi A and Nishinakamura R (2017) Higher-order kidney organogenesis from pluripotent stem cells. Cell Stem Cell 21:730-746.e6.

Takahashi K, Tanabe K, Ohnuki M, Narita M, Ichisaka T, Tomoda K, and Yamanaka S (2007) Induction of pluripotent stem cells from adult human fibroblasts by defined factors. Cell 131:861-872

Takahashi Y, Hori Y, Yamamoto T, Urashima T, Ohara Y, and Tanaka H (2015) 3D spheroid cultures improve the metabolic gene expression profiles of HepaRG cells. Biosci Rep 35:e0208

Takahashi Y, Sato S, Kurashima Y, Yamamoto T, Kurokawa S, Yuki Y, Takemura N, Uematsu S, Lai C-Y, Otsu M et al. (2018) A refined culture system for human induced pluripotent stem cell-derived intestinal epithelial organoids. Stem Cell Reports 10:314-328.
Takasato M, Er PX, Chiu HS, Maier B, Baillie GJ, Ferguson C, Parton RG, Wolvetang EJ, Roost MS, Chuva de Sousa Lopes SM et al. (2015) Kidney organoids from human iPS cells contain multiple lineages and model human nephrogenesis. Nature 526:564-568.

Takayama K, Kawabata K, Nagamoto Y, Kishimoto K, Tashiro K, Sakurai F, Tachibana M, Kanda K, Hayakawa T, Furue MK et al. (2013) 3D spheroid culture of hESC/hiPSC-derived hepatocyte-like cells for drug toxicity testing. Biomaterials 34:1781-1789.

Tan H-Y, Trier S, Rahbek UL, Dufva M, Kutter JP, and Andresen TL (2018) A multi-chamber microfluidic intestinal barrier model using Caco-2 cells for drug transport studies. PLoS One 13:e0197101.

Tan K, Keegan P, Rogers M, Lu M, Gosset JR, Charest J, and Bale SS (2019) A high-throughput microfluidic microphysiological system (PREDICT-96) to recapitulate hepatocyte function in dynamic, re-circulating flow conditions. Lab Chip 19:1556-1566.

Tanaka Y, Taki Y, Sakane T, Nadai T, Sezaki H, and Yamashita S (1995) Characterization of drug transport through tight-junctional pathway in Caco-2 monolayer: comparison with isolated rat jejunum and colon. Pharm Res 12:523-528.

Tang H, Hammack C, Ogden SC, Wen Z, Qian X, Li Y, Yao B, Shin J, Zhang F, Lee EM et al. (2016) Zika virus infects human cortical neural progenitors and attenuates their growth. Cell Stem Cell 18:587-590.

Tang-Schomer MD, White JD, Tien LW, Schmitt LI, Valentin TM, Graziano DJ, Hopkins AM, Omenetto FG, Haydon PG, and Kaplan DL (2014) Bioengineered functional brain-like cortical tissue. Proc Natl Acad Sci USA 111:13811-13816.

Tao Y and Zhang S-C (2016) Neural subtype specification from human pluripotent stem cells. Cell Stem Cell 19:573-586.

Tasnim F, Toh Y-C, Qu Y, Li H, Phan D, Narmada BC, Ananthanarayanan A, Mittal N, Meng RQ, and Yu H (2016) Functionally enhanced human stem cell derived hepatocytes in galactosylated cellulosic sponges for hepatotoxicity testing. Mol Pharm 13:1947-1957.

Tavelin S, Taipalensuu J, Hallböök F, Vellonen KS, Moore V, and Artursson P (2003a) An improved cell culture model based on 2/4/A1 cell monolayers for studies of intestinal drug transport: characterization of transport routes. Pharm Res 20:373-381.

Tavelin S, Taipalensuu J, Söderberg L, Morrison R, Chong S, and Artursson P (2003b) Prediction of the oral absorption of low-permeability drugs using small intestine-like 2/4/A1 cell monolayers. Pharm Res 20:397-405.

Teixeira MG, Costa MdaC, de Oliveira WK, Nunes ML, and Rodrigues LC (2016) The Epidemic of Zika virus-related microcephaly in Brazil: detection, control, etiology, and future scenarios. Am J Public Health 106:601-605.

Thavandiran N, Hale C, Blit P, Sandberg ML, McElvain ME, Gagliardi M, Sun B, Witty A, Graham G, Do VTH et al. (2020) Functional arrays of human pluripotent stem cell-derived cardiac microtissues. Sci Rep 10:6919.

Theobald J, Abu El Maaty MA, Kusterer N, Wetterauer B, Wink M, Cheng X, and Wölfl S (2019) In vitro metabolic activation of vitamin D3 by using a multicompartment microfluidic liver-kidney organ on chip platform. Sci Rep 9:4616.

Thompson PD, Clarkson PM, and Rosenson RS; National Lipid Association Statin Safety Task Force Muscle Safety Expert Panel (2006) An assessment of statin safety by muscle experts. Am J Cardiol 97 (8A):69C-76C.

Thomson A, Smart K, Somerville MS, Lauder SN, Appanna G, Horwood J, Sunder Raj L, Srivastava B, Durai D, Scurr MJ et al. (2019) The Ussing chamber system for measuring intestinal permeability in health and disease. BMC Gastroenterol 19:98-14.

Thorne CA, Chen IW, Sanman LE, Cobb MH, Wu LF, and Altschuler SJ (2018) Enteroid monolayers reveal an autonomous WNT and BMP circuit controlling intestinal epithelial growth and organization. Dev Cell 44:624-633.e4.

Thuenauer R, Müller SK, and Römer W (2017) Pathways of protein and lipid receptor-mediated transcytosis in drug delivery. Expert Opin Drug Deliv 14:341-351.

Tiong HY, Huang P, Xiong S, Li Y, Vathsala A, and Zink D (2014) Drug-induced nephrotoxicity: clinical impact and preclinical in vitro models. Mol Pharm 11:1933-1948.

Tohyama S, Hattori F, Sano M, Hishiki T, Nagahata Y, Matsuura T, Hashimoto H, Suzuki T, Yamashita H, Satoh Y et al. (2013) Distinct metabolic flow enables large-scale purification of mouse and human pluripotent stem cell-derived cardiomyocytes. Cell Stem Cell 12:127-137.

Tong JZ, De Lagausie P, Furlan V, Cresteil T, Bernard O, and Alvarez F (1992) Long-term culture of adult rat hepatocyte spheroids. Exp Cell Res 200:326-332.

Tostões RM, Leite SB, Serra M, Jensen J, Björquist P, Carrondo MJT, Brito C, and Alves PM (2012) Human liver cell spheroids in extended perfusion bioreactor culture for repeated-dose drug testing. Hepatology 55:1227-1236.

Trapecar M, Communal C, Velazquez J, Maass CA, Huang Y-J, Schneider K, Wright CW, Butty V, Eng G, Yilmaz O et al. (2020) Gut-liver physiomimetics reveal paradoxical modulation of IBD-related inflammation by short-chain fatty acids. Cell Syst 10:223-239.e9.

Trapecar M, Wogram E, Svoboda D, Communal C, Omer A, Lungjangwa T, Sphabmixay P, Velazquez J, Schneider K, Wright CW, Mildrum S, Hendricks A, Levine S, Muffat J, Lee MJ, Lauffenburger DA, Trumper D, Jaenisch R and Griffith LG (2021) Human physiomimetic model integrating microphysiological systems of the gut, liver, and brain for studies of neurodegenerative diseases. Science Advances 7:eabd1707.

Trietsch SJ, Israëls GD, Joore J, Hankemeier T, and Vulto P (2013) Microfluidic titer plate for stratified 3D cell culture. Lab Chip 13:3548-3554

Trietsch SJ, Naumovska E, Kurek D, Setyawati MC, Vormann MK, Wilschut KJ, Lanz HL, Nicolas A, Ng CP, Joore J et al. (2017) Membrane-free culture and real-time barrier integrity assessment of perfused intestinal epithelium tubes. Nat Commun 8:262. 
Tsamandouras N, Chen WLK, Edington CD, Stokes CL, Griffith LG, and Cirit M (2017) Integrated gut and liver microphysiological systems for quantitative in vitro pharmacokinetic studies. AAPS J 19:1499-1512.

Turowski P and Kenny B-A (2015) The blood-brain barrier and methamphetamine: open sesame? Front Neurosci 9:156.

Uchimura K, Wu H, Yoshimura Y, and Humphreys BD (2020) Human pluripotent stem cell-derived kidney organoids with improved collecting duct maturation and injury modeling. Cell Rep 33:108514.

Umehara K, Cantrill C, Wittwer MB, Di Lenarda E, Klammers F, Ekiciler A, Parrott N, Fowler S, and Ullah M (2020) Application of the extended clearance classification system (ECCS) in drug discovery and development: selection of appropriate in vitro tools and clearance prediction. Drug Metab Dispos 48:849-860.

Urich E, Patsch C, Aigner S, Graf M, Iacone R, and Freskgård P-O (2013) Multicellular self-assembled spheroidal model of the blood brain barrier. Sci Rep 3:1500.

Vacca M, Leslie J, Virtue S, Lam BYH, Govaere O, Tiniakos D, Snow S, Davies S, Petkevicius K, Tong Z et al. (2020) Bone morphogenetic protein $8 \mathrm{~B}$ promotes the progression of non-alcoholic steatohepatitis. Nat Metab 2:514-531.

van der Helm MW, van der Meer AD, Eijkel JCT, van den Berg A, and Segerink LI (2016) Microfluidic organ-on-chip technology for blood-brain barrier research. Tissue Barriers 4:e1142493.

van der Voorn SM, Te Riele ASJM, Basso C, Calkins H, Remme CA, and van Veen TAB (2020) Arrhythmogenic cardiomyopathy: pathogenesis, pro-arrhythmic remodelling, and novel approaches for risk stratification and therapy. Cardiovasc Res 116:1571-1584.

van Meer BJ, Krotenberg A, Sala L, Davis RP, Eschenhagen T, Denning C, Tertoolen LGJ, and Mummery CL (2019) Simultaneous measurement of excitation-contraction coupling parameters identifies mechanisms underlying contractile responses of hiPSC-derived cardiomyocytes. Nat Commun 10:4325-4329.

van Meer PJK, Kooijman M, Gispen-de Wied CC, Moors EHM, and Schellekens H (2012) The ability of animal studies to detect serious post marketing adverse events is limited. Regul Toxicol Pharmacol 64:345-349.

Van Norman GA (2019) Limitations of animal studies for predicting toxicity in clinical trials: is it time to rethink our current approach? JACC Basic Transl Sci 4:845-854.

Verheijen M, Schrooders Y, Gmuender H, Nudischer R, Clayton O, Hynes J, Niederer S, Cordes H, Kuepfer L, Kleinjans J et al. (2018) Bringing in vitro analysis closer to in vivo: studying doxorubicin toxicity and associated mechanisms in 3D human microtissues with PBPK-based dose modelling. Toxicol Lett 294:184-192.

Vernetti LA, Senutovitch N, Boltz R, DeBiasio R, Shun TY, Gough A, and Taylor DL (2016) A human liver microphysiology platform for investigating physiology, drug safety, and disease models. Exp Biol Med (Maywood) 241:101-114.

Versantvoort CH, Ondrewater RC, Duizer E, Van de Sandt JJ, Gilde AJ, and Groten JP (2002) Monolayers of IEC-18 cells as an in vitro model for screening the passive transcellular and paracellular transport across the intestinal barrier: comparison of active and passive transport with the human colon carcinoma Caco-2 cell line. Environ Toxicol Pharmacol 11:335-344.

Vigilante A, Laddach A, Moens N, Meleckyte R, Leha A, Ghahramani A, Culley OJ, Kathuria A, Hurling C, Vickers A et al.; HipSci Consortium (2019) Identifying extrinsic versus intrinsic drivers of variation in cell behavior in human iPSC lines from healthy donors. Cell Rep 26:2078-2087.e3.

Vila OF, Uzel SGM, Ma SP, Williams D, Pak J, Kamm RD, and Vunjak-Novakovic G (2019) Quantification of human neuromuscular function through optogenetics. Theranostics 9:1232-1246.

Villenave R, Wales SQ, Hamkins-Indik T, Papafragkou E, Weaver JC, Ferrante TC, Bahinski A, Elkins CA, Kulka M, and Ingber DE (2017) Human gut-on-a-chip supports polarized infection of Coxsackie B1 virus in vitro. PLoS One 12:e169412.

Vivares A, Salle-Lefort S, Arabeyre-Fabre C, Ngo R, Penarier G, Bremond M, Moliner P, Gallas J-F, Fabre G, and Klieber S (2015) Morphological behaviour and metabolic capacity of cryopreserved human primary hepatocytes cultivated in a perfused multiwell device. Xenobiotica 45:29-44.

von Keutz E and Schlüter G (1998) Preclinical safety evaluation of cerivastatin, a novel HMG-CoA reductase inhibitor. Am J Cardiol 82 (4B):11J-17J.

Vormann MK, Gijzen L, Hutter S, Boot L, Nicolas A, van den Heuvel A, Vriend J, Ng CP, Nieskens TTG, van Duinen V et al. (2018) Nephrotoxicity and kidney transport assessment on 3D perfused proximal tubules. AAPS J 20:90.

Vormann MK, Vriend J, Lanz HL, Gijzen L, van den Heuvel A, Hutter S, Joore J, Trietsch SJ, Stuut C, Nieskens TTG et al. (2021) Implementation of a human renal proximal tubule on a chip for nephrotoxicity and drug interaction studies. J Pharm Sci 110:1601-1614.

Vorrink SU, Ullah S, Schmidt S, Nandania J, Velagapudi V, Beck O, IngelmanSundberg M, and Lauschke VM (2017) Endogenous and xenobiotic metabolic stability of primary human hepatocytes in long-term 3D spheroid cultures revealed by a combination of targeted and untargeted metabolomics. FASEB $J$ 31:2696-2708.

Vorrink SU, Zhou Y, Ingelman-Sundberg M, and Lauschke VM (2018) Prediction of drug-induced hepatotoxicity using long-term stable primary hepatic $3 \mathrm{D}$ spheroid cultures in chemically defined conditions. Toxicol Sci 163:655-665.

Vriend J, Nieskens TTG, Vormann MK, van den Berge BT, van den Heuvel A, Russel FGM, Suter-Dick L, Lanz HL, Vulto P, Masereeuw R et al. (2018) Screening of drug-transporter interactions in a 3D microfluidic renal proximal tubule on a chip. AAPS J 20:87.

Vriend J, Peters JGP, Nieskens TTG, Škovroňová R, Blaimschein N, Schmidts M, Roepman R, Schirris TJJ, Russel FGM, Masereeuw R et al. (2020) Flow stimulates drug transport in a human kidney proximal tubule-on-a-chip independent of primary cilia. Biochim Biophys Acta, Gen Subj 1864:129433.

Vriend J, Pye KR, and Brown C (2021) In vitro models for accurate prediction of renal tubular xenobiotic transport in vivo. Curr Opin Toxicol 25:15-22.
Vulto P, Podszun S, Meyer P, Hermann C, Manz A, and Urban GA (2011) Phaseguides: a paradigm shift in microfluidic priming and emptying. Lab Chip 11:1596-1602

Walter E and Kissel T (1995) Heterogeneity in the human intestinal-cell line Caco2 leads to differences in transepithelial transport. Eur J Pharm Sci 3:215-230.

Wang C, Gao X, Mawatari K, and Kitamori T (2017a) Clogging-free irreversible bonding of polycarbonate membranes to glass microfluidic devices. J Electrochem Soc 164:B3087.

Wang G, McCain ML, Yang L, He A, Pasqualini FS, Agarwal A, Yuan H, Jiang D, Zhang D, Zangi L et al. (2014) Modeling the mitochondrial cardiomyopathy of Barth syndrome with induced pluripotent stem cell and heart-on-chip technologies. Nat Med 20:616-623.

Wang J, Chen F, Liu L, Qi C, Wang B, Yan X, Huang C, Hou W, Zhang MQ, Chen Y et al. (2016) Engineering EMT using 3D micro-scaffold to promote hepatic functions for drug hepatotoxicity evaluation. Biomaterials 91:11-22.

Wang J, Khodabukus A, Rao L, Vandusen K, Abutaleb N, and Bursac N (2019a) Engineered skeletal muscles for disease modeling and drug discovery. Biomaterials 221:119416.

Wang JH-C and Lin J-S (2007) Cell traction force and measurement methods. Biomech Model Mechanobiol 6:361-371.

Wang S, Bates J, Li X, Schanz S, Chandler-Militello D, Levine C, Maherali N, Studer L, Hochedlinger K, Windrem M et al. (2013) Human iPSC-derived oligodendrocyte progenitor cells can myelinate and rescue a mouse model of congenital hypomyelination. Cell Stem Cell 12:252-264.

Wang WW, Khetani SR, Krzyzewski S, Duignan DB, and Obach RS (2010) Assessment of a micropatterned hepatocyte coculture system to generate major human excretory and circulating drug metabolites. Drug Metab Dispos 38:1900-1905.

Wang X, Cirit M, Wishnok JS, Griffith LG, and Tannenbaum SR (2019b) Analysis of an integrated human multiorgan microphysiological system for combined tolcapone metabolism and brain metabolomics. Anal Chem 91:8667-8675.

Wang Y, DiSalvo M, Gunasekara DB, Dutton J, Proctor A, Lebhar MS, Williamson IA, Speer J, Howard RL, Smiddy NM et al. (2017b) Self-renewing monolayer of primary colonic or rectal epithelial cells. Cell Mol Gastroenterol Hepatol 4:165-182.e7.

Wang Y, Gunasekara DB, Reed MI, DiSalvo M, Bultman SJ, Sims CE, Magness ST, and Allbritton NL (2017c) A microengineered collagen scaffold for generating a polarized crypt-villus architecture of human small intestinal epithelium. Biomaterials 128:44-55.

Wang Y, Wang H, Deng P, Chen W, Guo Y, Tao T, and Qin J (2018a) In situ differentiation and generation of functional liver organoids from human iPSCs in a 3D perfusable chip system. Lab Chip 18:3606-3616.

Wang Y, Wang L, Zhu Y, and Qin J (2018b) Human brain organoid-on-a-chip to model prenatal nicotine exposure. Lab Chip 18:851-860.

Wang Z, Luo X, Anene-Nzelu C, Yu Y, Hong X, Singh NH, Xia L, Liu S, and Yu H (2015) HepaRG culture in tethered spheroids as an in vitro three-dimensional model for drug safety screening. J Appl Toxicol 35:909-917.

Ward KW, Nagilla R, and Jolivette LJ (2005) Comparative evaluation of oral systemic exposure of 56 xenobiotics in rat, dog, monkey and human. Xenobiotica 35:191-210.

Ware BR, Berger DR, and Khetani SR (2015) Prediction of drug-induced liver injury in micropatterned co-cultures containing iPSC-derived human hepatocytes. Toxicol Sci 145:252-262.

Ware BR, Durham MJ, Monckton CP, and Khetani SR (2017) A cell culture platform to maintain long-term phenotype of primary human hepatocytes and endothelial cells. Cell Mol Gastroenterol Hepatol 5:187-207.

Waring MJ, Arrowsmith J, Leach AR, Leeson PD, Mandrell S, Owen RM, Pairaudeau G, Pennie WD, Pickett SD, Wang J et al. (2015) An analysis of the attrition of drug candidates from four major pharmaceutical companies. Nat Rev Drug Discov 14:475-486.

Watkins PB (2011) Drug safety sciences and the bottleneck in drug development. Clin Pharmacol Ther 89:788-790.

Weber EJ, Chapron A, Chapron BD, Voellinger JL, Lidberg KA, Yeung CK, Wang Z, Yamaura Y, Hailey DW, Neumann T et al. (2016) Development of a microphysiological model of human kidney proximal tubule function. Kidney Int 90:627-637.

Weber EJ, Lidberg KA, Wang L, Bammler TK, MacDonald JW, Li MJ, Redhair M, Atkins WM, Tran C, Hines KM et al. (2018) Human kidney on a chip assessment of polymyxin antibiotic nephrotoxicity. JCI Insight 3:e123673.

Wienkers LC and Heath TG (2005) Predicting in vivo drug interactions from in vitro drug discovery data. Nat Rev Drug Discov 4:825-833.

Wikswo JP, Curtis EL, Eagleton ZE, Evans BC, Kole A, Hofmeister LH, and Matloff WJ (2013) Scaling and systems biology for integrating multiple organson-a-chip. Lab Chip 13:3496-3511.

Williamson IA Arnold JW, Samsa LA, Gaynor L, DiSalvo M, Cocchiaro JL, Carroll I, Azcarate-Peril MA, Rawls JF, Allbritton NL et al. (2018) A high-throughput organoid microinjection platform to study gastrointestinal microbiota and luminal physiology. Cell Mol Gastroenterol Hepatol 6:301-319.

Workman MJ, Gleeson JP, Troisi EJ, Estrada HQ, Kerns SJ, Hinojosa CD, Hamilton GA, Targan SR, Svendsen CN, and Barrett RJ (2017) Enhanced utilization of induced pluripotent stem cell-derived human intestinal organoids using microengineered chips. Cell Mol Gastroenterol Hepatol 5:669-677.e2.

Wu H, Uchimura K, Donnelly EL, Kirita Y, Morris SA, and Humphreys BD (2018) Comparative analysis and refinement of human PSC-derived kidney organoid differentiation with single-cell transcriptomics. Cell Stem Cell 23:869-881.e8.

Xi B, Wang T, Li N, Ouyang W, Zhang W, Wu J, Xu X, Wang X, and Abassi YA (2011) Functional cardiotoxicity profiling and screening using the xCELLigence RTCA cardio system. $J$ Lab Autom 16:415-421.

Xia Y and Whitesides GM (1998) Soft lithography. Angew Chem Int Ed Engl 37:550-575. 
Xiang C, Du Y, Meng G, Soon Yi L, Sun S, Song N, Zhang X, Xiao Y, Wang J, Yi Z et al. (2019) Long-term functional maintenance of primary human hepatocytes in vitro. Science 364:399-402.

Xu JJ, Henstock PV, Dunn MC, Smith AR, Chabot JR, and de Graaf D (2008) Cellular imaging predictions of clinical drug-induced liver injury. Toxicol Sci 105:97-105.

Xu T, Molnar P, Gregory C, Das M, Boland T, and Hickman JJ (2009) Electrophysiological characterization of embryonic hippocampal neurons cultured in a $3 \mathrm{D}$ collagen hydrogel. Biomaterials 30:4377-4383.

Yadid M, Lind JU, Ardoña HAM, Sheehy SP, Dickinson LE, Eweje F, Bastings MMC, Pope B, O'Connor BB, Straubhaar JR et al. (2020) Endothelial extracellular vesicles contain protective proteins and rescue ischemia-reperfusion injury in a human heart-on-chip. Sci Transl Med 12:eaax8005.

Yajima Y, Lee CN, Yamada M, Utoh R, and Seki M (2018) Development of a perfusable 3D liver cell cultivation system via bundling-up assembly of cell-laden microfibers. J Biosci Bioeng 126:111-118.

Yang C, Yin X-H, and Cheng G-M (2013) Microinjection molding of microsystem components: new aspects in improving performance. $J$ Micromech Microeng 23:093001.

Yang N, Ng YH, Pang ZP, Südhof TC, and Wernig M (2011) Induced neuronal cells: how to make and define a neuron. Cell Stem Cell 9:517-525.

Yavarpour-Bali H, Nakhaei-Nejad M, Yazdi A, and Ghasemi-Kasman M (2020) Direct conversion of somatic cells towards oligodendroglial lineage cells: a nove strategy for enhancement of myelin repair. J Cell Physiol 235:2023-2036.

Ye F, Kang E, Yu C, Qian X, Jacob F, Yu C, Mao M, Poon RYC, Kim J, Song H et al. (2017) DISC1 regulates neurogenesis via modulating kinetochore attachment of Ndel1/Nde1 during mitosis. Neuron 96:1041-1054.e5.

Yin F, Zhang X, Wang L, Wang Y, Zhu Y, Li Z, Tao T, Chen W, Yu H, and Qin J (2021) HiPSC-derived multi-organoids-on-chip system for safety assessment of antidepressant drugs. Lab Chip 21:571-581.

Yokoyama T, Shimizu M, Ohta K, Yuno T, Okajima M, Wada T, Toma T, Koizumi S, and Yachie A (2011) Urinary heme oxygenase-1 as a sensitive indicator of tubulointerstitial inflammatory damage in various renal diseases. Am J Nephrol 33:414-420.

Yokoyama Y, Sasaki Y, Terasaki N, Kawataki T, Takekawa K, Iwase Y, Shimizu T, Sanoh S, and Ohta S (2018) Comparison of drug metabolism and its related hepatotoxic effects in HepaRG, cryopreserved human hepatocytes, and HepG2 cell cultures. Biol Pharm Bull 41:722-732.

Yoon KJ, Song G, Qian X, Pan J, Xu D, Rho H-S, Kim N-S, Habela C, Zheng L, Jacob $\mathrm{F}$ et al. (2017) Zika-virus-encoded NS2A disrupts mammalian cortical neurogenesis by degrading adherens junction proteins. Cell Stem Cell 21:349-358.e6.

Yoshida S, Miwa H, Kawachi T, Kume S, and Takahashi K (2020) Generation of intestinal organoids derived from human pluripotent stem cells for drug testing. Sci Rep 10:5989.

Youhanna S and Lauschke VM (2021) The past, present and future of intestinal in vitro cell systems for drug absorption studies. J Pharm Sci 110:50-65.

Youhanna S, Wright SC, and Lauschke VM (2021) Organotypic human ex vivo models for coronavirus disease 2019 research and drug development. Curr Opin Pharmacol 59:11-18.

Younossi ZM, Koenig AB, Abdelatif D, Fazel Y, Henry L, and Wymer M (2016) Global epidemiology of nonalcoholic fatty liver disease-Meta-analytic assessment of prevalence, incidence, and outcomes. Hepatology 64:73-84.

Yu F, Deng R, Hao Tong W, Huan L, Chan Way N, IslamBadhan A, Iliescu C, and Yu H (2017) A perfusion incubator liver chip for 3D cell culture with application on chronic hepatotoxicity testing. Sci Rep 7:14528.

Yu M-Y, Kim JE, Lee S, Choi JW, Kim YC, Han SS, Lee H, Cha RH, Lee JP, Lee JW et al. (2020) Krüppel-like factor 15 is a key suppressor of podocyte fibrosis under rotational force-driven pressure. Exp Cell Res 386:111706.

Zachos NC, Kovbasnjuk O, Foulke-Abel J, In J, Blutt SE, de Jonge HR, Estes MK and Donowitz M (2016) Human enteroids/colonoids and intestinal organoids functionally recapitulate normal intestinal physiology and pathophysiology. $J$ Biol Chem 291:3759-3766.

Zager RA, Johnson ACM, and Becker K (2012) Plasma and urinary heme oxygenase-1 in AKI. J Am Soc Nephrol 23:1048-1057.

Zahavi EE, Ionescu A, Gluska S, Gradus T, Ben-Yaakov K, and Perlson E (2015) A compartmentalized microfluidic neuromuscular co-culture system reveals spatia aspects of GDNF functions. J Cell Sci 128:1241-1252.

Zandi Shafagh R, Decrop D, Ven K, Vanderbeke A, Hanusa R, Breukers J, Pardon G, Haraldsson T, Lammertyn J, and van der Wijngaart W (2019) Reaction injection molding of hydrophilic-in-hydrophobic femtolitre-well arrays. Microsyst Nanoeng 5:25.

Zandi Shafagh R, Shen JX, Youhanna S, Guo W, Lauschke VM, van der Wijngaart $\mathrm{W}$, and Haraldsson T (2020) Facile nanoimprinting of robust high-aspect-ratio nanostructures for human cell biomechanics. ACS Appl Bio Mater 3:8757-8767.

Zandi Shafagh R, van der Wijngaart W, and Haraldsson T(2017) NANORIM: Submicron structuring with reaction injection molding, IEEE 30th International Conference on Micro Electro Mechanical Systems (MEMS) 213-216.

Zandi Shafagh R, Vastesson A, Guo W, van der Wijngaart W, and Haraldsson T (2018) E-Beam nanostructuring and direct click biofunctionalization of Thiol-Ene resist. ACS Nano 12:9940-9946.

Zhang C, Zhao Z, Abdul Rahim NA, van Noort D, and Yu H (2009) Towards a human-on-chip: culturing multiple cell types on a chip with compartmentalized microenvironments. Lab Chip 9:3185-3192.

Zhang SC, Wernig M, Duncan ID, Brüstle O, and Thomson JA (2001) In vitro differentiation of transplantable neural precursors from human embryonic stem cells. Nat Biotechnol 19:1129-1133.

Zhang W, Zhang YS, Bakht SM, Aleman J, Shin SR, Yue K, Sica M, Ribas J, Duchamp M, Ju J et al. (2016b) Elastomeric free-form blood vessels for interconnecting organs on chip systems. Lab Chip 16:1579-1586.

Zhang X, Jiang T, Chen D, Wang Q, and Zhang LW (2020) Three-dimensional liver models: state of the art and their application for hepatotoxicity evaluation. Crit Rev Toxicol 50:279-309.

Zhang YS, Aleman J, Shin SR, Kilic T, Kim D, Mousavi Shaegh SA, Massa S, Riah $\mathrm{R}$, Chae S, Hu N et al. (2017) Multisensor-integrated organs-on-chips platform for automated and continual in situ monitoring of organoid behaviors. Proc Natl Acad Sci USA 114:E2293-E2302.

Zhang Y, Sloan SA, Clarke LE, Caneda C, Plaza CA, Blumenthal PD, Vogel H, Steinberg GK, Edwards MS, Li G et al. (2016a) Purification and characterization of progenitor and mature human astrocytes reveals transcriptional and functional differences with mouse. Neuron 89:37-53.

Zhao Y, Rafatian N, Feric NT, Cox BJ, Aschar-Sobbi R, Wang EY, Aggarwal P, Zhang B, Conant G, Ronaldson-Bouchard K et al. (2019) A platform for generation of chamber-specific cardiac tissues and disease modeling. Cell 176:913-927.e18.

Zhou J, Li C, Liu X, Chiu MC, Zhao X, Wang D, Wei Y, Lee A, Zhang AJ, Chu H et al. (2020) Infection of bat and human intestinal organoids by SARS-CoV-2. Nat Med 26:1077-1083.

Zhou Y, Shen JX, and Lauschke VM (2019) Comprehensive evaluation of organotypic and microphysiological liver models for prediction of drug-induced liver injury. Front Pharmacol 10:1093.

Zhu Y, Wang L, Yin F, Yu Y, Wang Y, Shepard MJ, Zhuang Z, and Qin J (2017) Probing impaired neurogenesis in human brain organoids exposed to alcohol. Integr Biol 9:968-978.

Zou L, Stecula A, Gupta A, Prasad B, Chien H-C, Yee SW, Wang L, Unadkat JD, Stahl SH, Fenner KS et al. (2018) Molecular mechanisms for species differences in organic anion transporter 1, OAT1: implications for renal drug toxicity. Mol Pharmacol 94:689-699.

Zuppinger C (2017) Edge-detection for contractility measurements with cardiac spheroids, in Methods in Pharmacology and Toxicology, pp 211-227, Humana Press, New York, NY DOI: https://doi.org/10.1007/978-1-4939-6661-5_11. 